\title{
Using Machine Learning to Refine Black-box Test Specifications and Test Suites
}

\author{
By
}

\section{Zaheer Bawar}

\author{
A thesis submitted to \\ the Faculty of Graduate Studies and Research \\ in partial fulfillment of \\ the requirements for the degree of \\ MASTER OF COMPUTER SCIENCE \\ at \\ Ottawa-Carleton Institute of Computer Science \\ School of Computer Science \\ CARLETON UNIVERSITY \\ Ottawa, Ontario, K1S 5B6
}

May 2009

Copyright $\odot 2009$ by Zaheer Bawar 


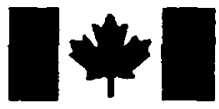

Library and Archives

Canada

Published Heritage

Branch

395 Wellington Street

Ottawa ON K1A 0N4

Canada
Bibliotheque et

Archives Canada

Direction du

Patrimoine de l'édition

395 , rue Wellington

Ottawa ON K1A 0N4

Canada
NOTICE:

The author has granted a nonexclusive license allowing Library and Archives Canada to reproduce, publish, archive, preserve, conserve, communicate to the public by telecommunication or on the Internet, loan, distribute and sell theses worldwide, for commercial or noncommercial purposes, in microform, paper, electronic and/or any other formats.

The author retains copyright ownership and moral rights in this thesis. Neither the thesis nor substantial extracts from it may be printed or otherwise reproduced without the author's permission.
Your file Votre référence

ISBN: 978-0-494-60241-6

Our file Notre référence

ISBN: 978-0-494-60241-6
In compliance with the Canadian Privacy Act some supporting forms may have been removed from this thesis.

While these forms may be included in the document page count, their removal does not represent any loss of content from the thesis.
L'auteur conserve la propriété du droit d'auteur et des droits moraux qui protège cette thèse. Ni la thèse ni des extraits substantiels de celle-ci ne doivent être imprimés ou autrement reproduits sans son autorisation.

AVIS:

L'auteur a accordé une licence non exclusive permettant à la Bibliothèque et Archives Canada de reproduire, publier, archiver, sauvegarder, conserver, transmettre au public par télécommunication ou par l'Internet, prêter, distribuer et vendre des thèses partout dans le monde, à des fins commerciales ou autres, sur support microforme, papier, électronique et/ou autres formats.

Conformément à la loi canadienne sur la protection de la vie privée, quelques formulaires secondaires ont été enlevés de cette thèse.

Bien que ces formulaires aient inclus dans la pagination, il n'y aura aucun contenu manquant.

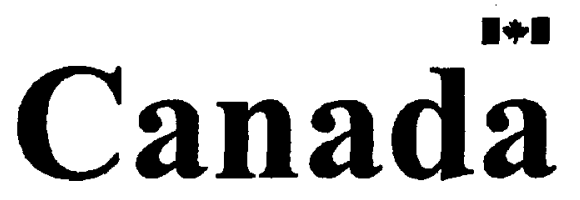




\begin{abstract}
In the context of open source development or software evolution, developers are often faced with potentially large test suites which have been developed with no apparent rationale and which may need to be augmented or refined to ensure sufficient dependability, or even possibly reduced to meet tight deadlines. We will refer to this process as the re-engineering of test suites. It is important to provide both a method and tool support to help people understand the limitations of test suites and their possible redundancies, so as to be able to re-engineer them in a cost effective manner. To address this problem in the case of black-box testing, we propose a method based on machine learning that has shown promising results on two case studies.
\end{abstract}




\section{Acknowledgments}

This piece of work would not have been accomplished without the people behind my life for inspiring, guiding and accompanying me through thick and thin.

It is difficult to overstate my gratitude to my supervisor, Dr Yvan Labiche, and cosupervisor, Dr. Lionel Briand, who has supported me thoughout my thesis with patience and knowledge whilst allowing me the room to work in my own way, I attribute the level of my Masters degree to their encouragement and effort.

I would like to express the deepest appreciation to my best friend, my mentor, and my guide Dr. Ghotai Manalai, whose continous support, love, and guidance in the ups and downs of my life made all this possible.

I am as ever, especially indebted to my wife Shaima for her love and support. Without her encouragement and understanding it would have been impossible for me to finish this work. My heartfelt gratitude to my family, my brothers, sisters, my uncles, and cousins, in particular, I must acknowledge Dr. Partamin Manalai, Dr. Partam Manalai, and Dr. Najeeb-u-Rahman Manalai for teaching me the things I would have never learned on my

own. I would also extend special thanks to my best friends Abdul Basir, Wasman Tasmim, Reymes Madrazo Rivera, Kaukaba Homam, Ahmad Shekib Ludin, Obaid Ghazniwal, Razia Ludin, Arezo Yousufzai, and Husnia Husnia.

To the Bishop's/Champlain Refugee-Student Sponsorship Project members, especially to Mary Purkey, Heather Thomson, Lisa McRae, Linda Allen, and, and to the rest, whom I 
failed to mention, without whose support God knows where I would have ended up. The financial support of the Bishop's University is gratefully acknowledged as well.

To all my friends at the Squall Lab, I would like to offer my sincerest thanks for providing a very friendly and supportive environement.

To everybody that has been a part of my life but I failed to mention, thank you very much. There won't be enough space if I mention you all.

Lastly, and most importantly, I wish to thank my parents. They bore me, raised me, supported me, taught me, and loved me. To them I dedicate this thesis. 


\section{Table of Contents}

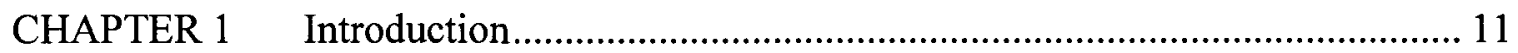

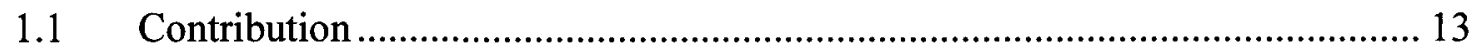

1.2 Thesis Organization ............................................................................ 13

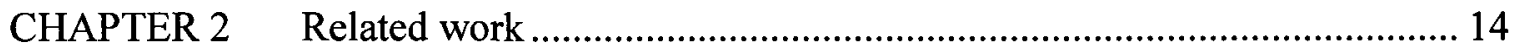

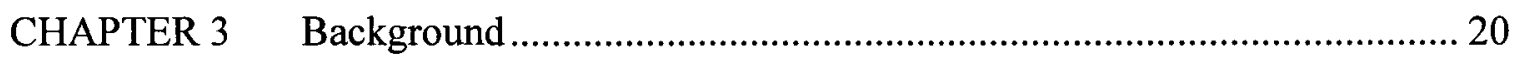

3.1 Using Category Partition................................................................................ 20

$3.2 \quad$ C4.5 Decision Trees (DT) ................................................................... 23

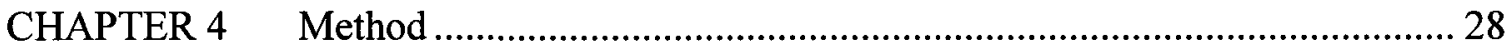

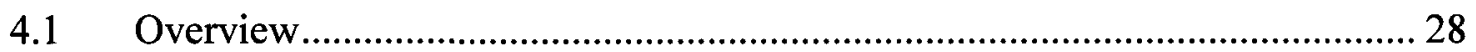

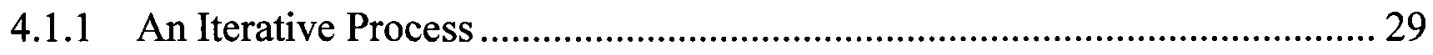

4.1.2 Manual Effort and Automation.................................................................. 32

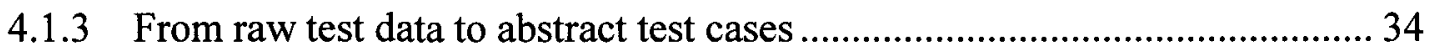

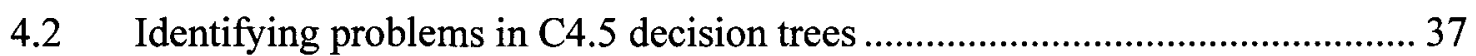

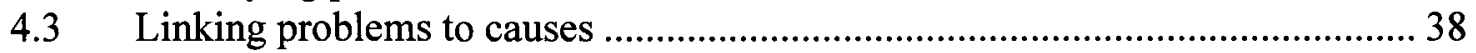

4.3.1 Case 1.1 - Missing category/choice ............................................................ 40

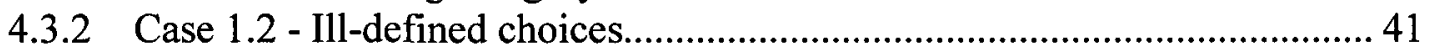

4.3.3 Case 2.1 - Useless category or choice...................................................... 42

4.3.4 Case 2.2 - Missing test cases..................................................................... 43

4.3.5 Case 3.1 - Unfeasible combinations............................................................... 43

4.3.6 Case 4.1 - Redundant Test Cases............................................................. 44

4.4 Heuristics for Refining Test Suite............................................................. 44

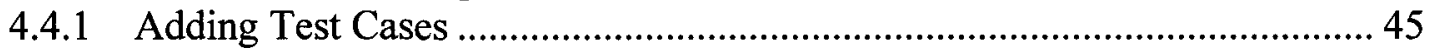

4.4.2 Removing Test Cases................................................................................. 47

CHAPTER 5 Case study one: Packhexchar............................................................ 48

5.1 The PackHexChar Program ........................................................................... 48

5.2 Application of Category-Partitioning …...................................................... 49

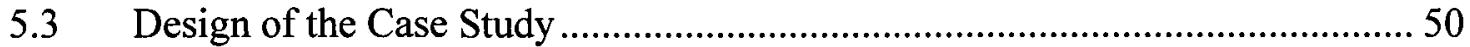

5.4 Comparing Iteration results with Experts' ...................................................... 52

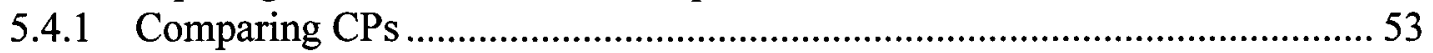

5.4.2 CP specification comparison: an example …….......................................56

5.5 Results with Student's CP-Evolution Context ............................................... 58

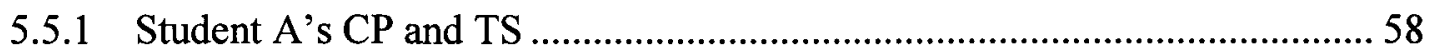

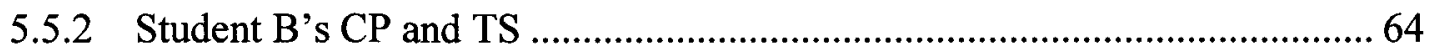

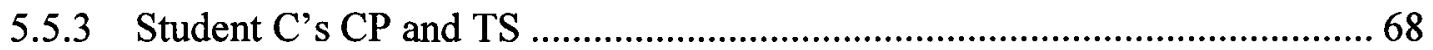

5.6 Results with the Expert CP - OSS Context ................................................ 72

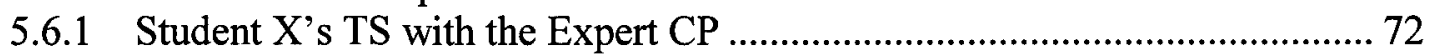

5.6.2 Student Y's TS with the Expert CP ............................................................ 77

5.6.3 Student Z's TS with the Expert CP ................................................................. 81

5.7 Assessing the fault detection effectiveness of improved test suites .................. 85

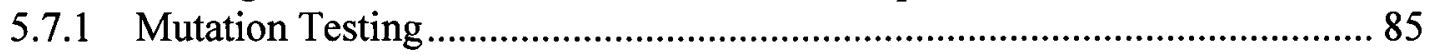




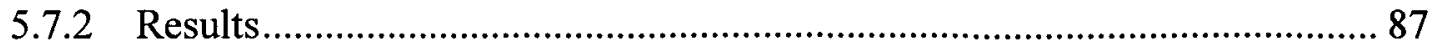

5.8 Analysis of alive mutants after test suite improvement/reduction.................... 91

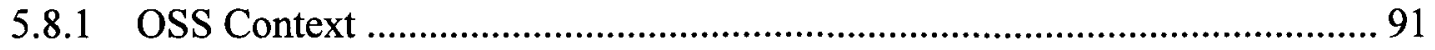

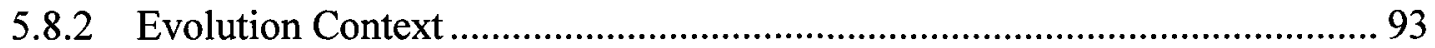

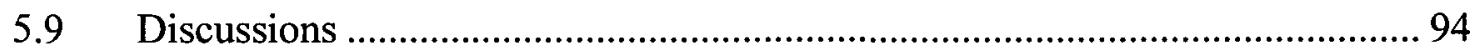

CHAPTER 6 Case study two: Space program......................................................... 97

6.1 The Space Program.................................................................................. 97

6.2 Design of the Experiment ............................................................................. 98

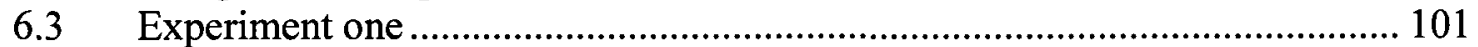

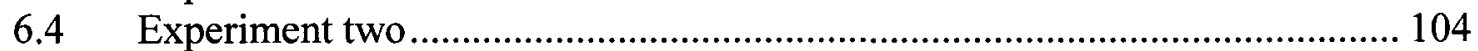

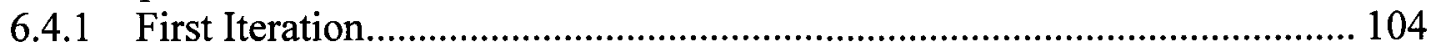

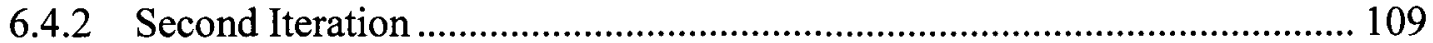

6.5 Melba Process Automation - PackHexChar and Space ................................... 112

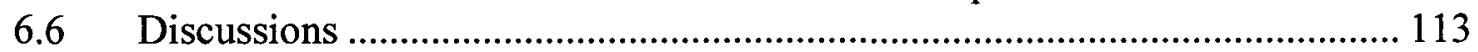

CHAPTER 7 Conslusions and Future work......................................................... 115

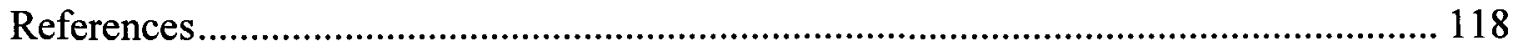

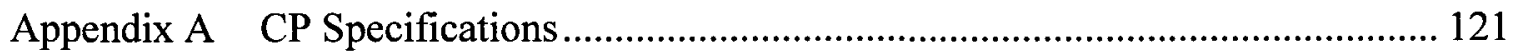

Appendix B Mapping of Categories/Choices of Expert CP and Student CPs ............ 131

Appendix C PackHexChar: Decision Trees before TS Reduction............................. 133

Appendix D Mutation Results-PackHexChar Program ............................................. 138

Appendix E The Space Program ............................................................................... 143 


\section{List of Figures}

Figure 1 Triangle Program CP Specification.............................................................. 22

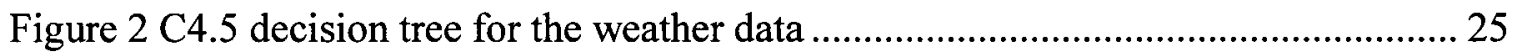

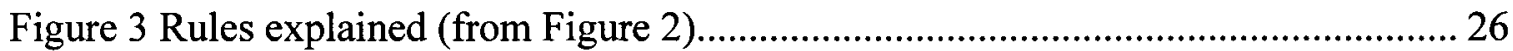

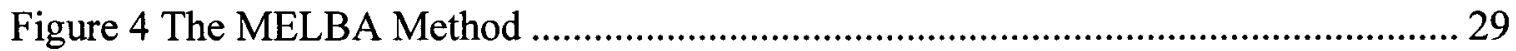

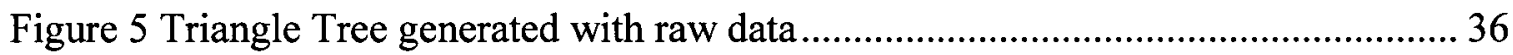

Figure 6 Triangle Tree generated with abstract test data .............................................. 37

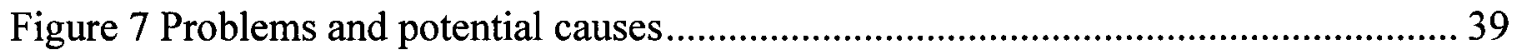

Figure 8 Examples using the Triangle Program ……….............................................. 41

Figure 9 Confusion matrix for the Triangle Program with Category 9 removed ............. 41

Figure 10 Adding Test Cases from the Tree .............................................................. 46

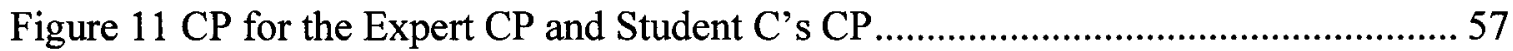

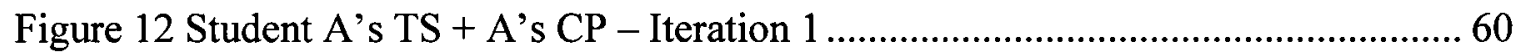

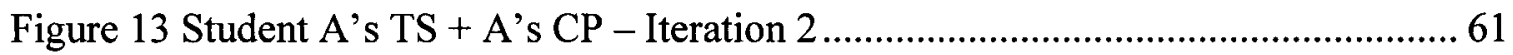

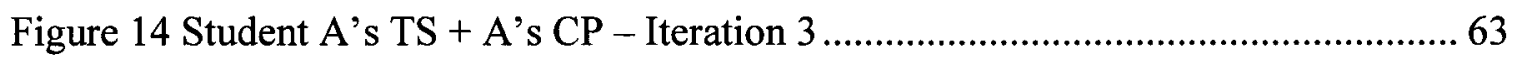

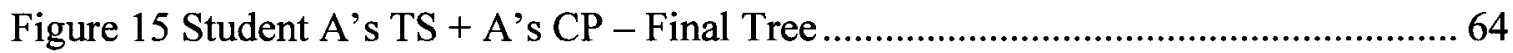

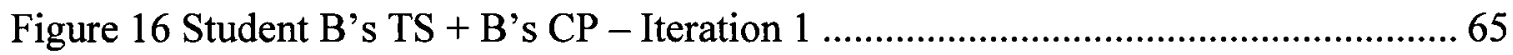

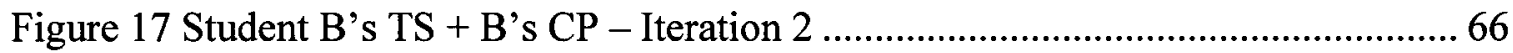

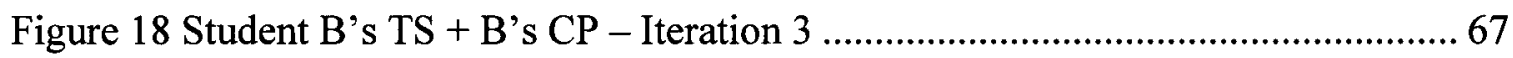

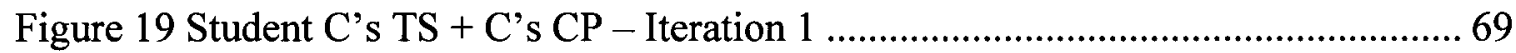

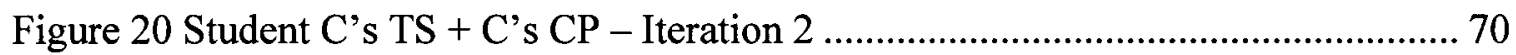

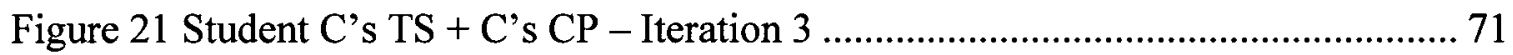

Figure 22 Student C's TS + C's CP - Final Tree …................................................ 71

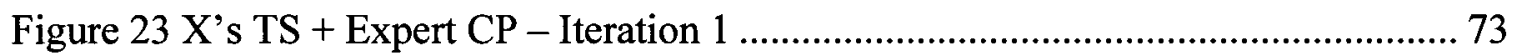

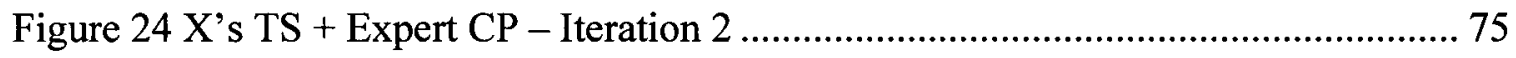

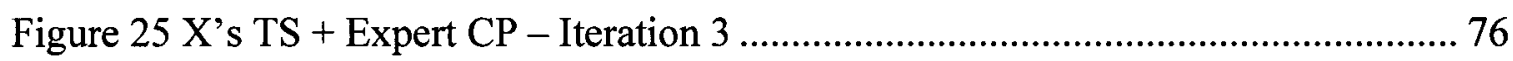

Figure 26 X's TS + Expert CP - Final Tree ………............................................... 77

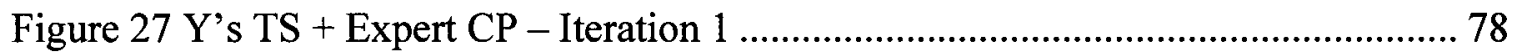

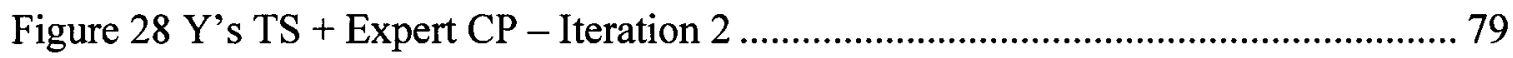

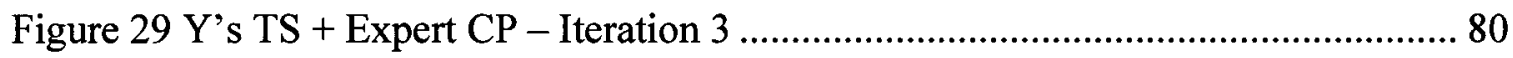

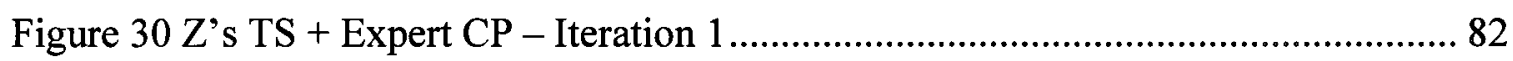

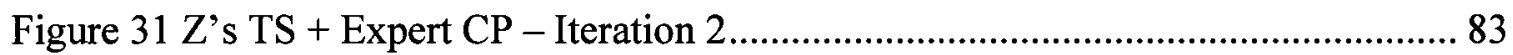

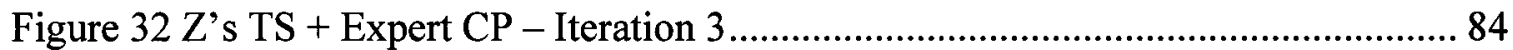

Figure 33 Number of (non-) Equivalent Mutants for each Mutation Operator ................ 87

Figure 34 Normal distribution of test cases per rule................................................. 102

Figure 35 Decision Tree with complete TS and Original CP - 16 Equivalence classes 103 
Figure 36 Decision Tree with reduced TS and Original CP - 16 Equivalence classes .. 106 Figure 37 Missing combinations of choices for TC's are added .................................. 108 Figure 38 Space Decision Tree - Second Iteration....................................................... 111 


\section{List of Tables}

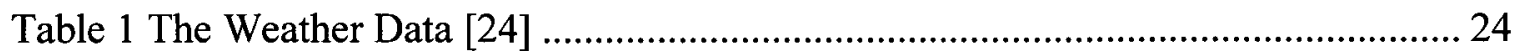

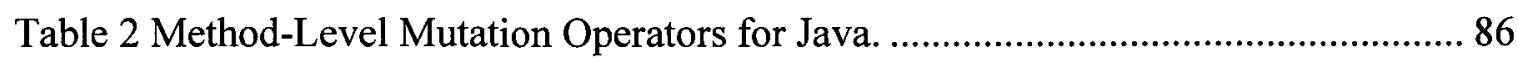

Table 3 Mutants killed and coverage by each test suite.................................................. 88

Table 4 Test Cases for the Triangle Program .............................................................. 123

Table 5 Abstract Test Cases using the Test Cases of Table 4 and $\mathrm{CP}$ specification of

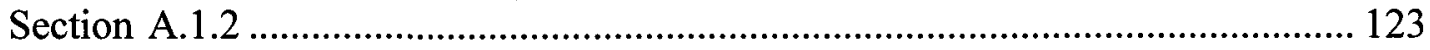

Table 6 Mutants not killed in the last two iterations for all students .............................. 138

Table 7 Mutants not killed by reduced student X TS ................................................ 138

Table 8 Mutants not killed by reduced student Y TS .................................................. 139

Table 9 Mutants not killed by reduced student Z TS..................................................... 139

Table 10 Mutants not killed by reduced student A TS ................................................. 139

Table 11 Mutants not killed by reduced student B TS................................................... 140

Table 12 Mutants not killed by reduced student C TS................................................ 142

Table 13 Mutants not killed by reduced Expert TS ........................................................ 142 


\section{CHAPTER 1 INTRODUCTION}

Software Testing is probably the most complex task in the software development cycle. The purpose of software testing is to increase the software engineers' confidence in the proper functioning of the software [1]. A test is the act of exercising software with test cases. Each choice of input data is called a test case. To identify test cases, functional and structural testing techniques are used [2].

In the context of open source development, it is often the case that one is confronted with existing test suites that are based on no explicit rationale or specifications, other than general guidelines for exercising the main user functionalities for example (e.g., based on check lists [3]). For instance, open source software development projects have been shown to lack "attention to basic, accepted, and mature testing techniques [4]." In practice, software developers who intend to reuse open source code are commonly confronted with such ad hoc test suites. It is therefore important to evaluate them and possibly reduce or augment them, depending on whether they are deemed redundant or too weak to achieve a sufficient level of confidence. For instance, Zhao and Elbaum report that in a large proportion of open source software development projects, test suites achieve low source code coverage (e.g., 30\%) [4]. Developers hence have an alternative: either build new test suites or reuse existing ones; and they often go for the latter as it (hopefully) reduces effort and therefore costs. This will inevitably lead them to understanding, evaluating and possibly improving these test suites. 
Similarly, in an evolution context, because of personnel turnover, the originator of a test suite may not be available and whoever is in charge of modifying and re-testing the software is confronted with understanding and evaluating existing test cases. Even in the context of regression testing, where one needs to select a subset and prioritize existing test cases, it is important to ensure that the original test suite is sufficiently complete and not redundant to minimize the cost.

We propose a partially automated method, based on the application of a machine learning technique, to help software engineers analyze the weaknesses of test suites so as to be able to iteratively improve them. We refer to this process as the reengineering of test suites as it is similar to what can be seen in re-engineering source code where code information is extracted, abstracted from a design standpoint, and then used to decide about design changes [5].

Our method takes as inputs the test suite, and test specification following the Category-Partition (CP) strategy [6] to be re-engineered. Based on the CP specification, test cases in the test suite are transformed into abstract test cases which are tuples of pairs (category, choice) associated with an output equivalence class (instead of raw inputs/outputs). A machine learning algorithm (C4.5 decision tree algorithm [7]) is used to learn rules that relate pairs (category, choice), modeling input properties, to output equivalence classes. These rules are then analyzed to determine potential improvements to the test suite (e.g., redundant test cases, need for additional test cases) as well as improvements to the CP specification (e.g., need to add a category). The method is applied to a small size but logically complex program (the PackHexChar program), and its effectiveness is evaluated on test suites and CP 
specifications created by fully trained $4^{\text {th }}$ year students. The study shows that the iterative process can indeed improve both the test suite and the CP specification to a level that is equivalent to what an expert would likely produce. The method is then applied to an industrial size program, thereby illustrating that it can scale up. Results are alo encouraging.

\subsection{Contribution}

The contributions of this thesis are:

- An iterative, incremental re-engineering process to evaluate and improve a test suite and test specification;

- The identification/definition of clear evaluation criteria;

- The definition of clear test suite improvement mechanisms;

- The evaluation of the proposed method on two case studies;

- Tool support for the case studies.

\subsection{Thesis Organization}

The rest of the thesis is structured as follows. Related work is described in CHAPTER 2. CHAPTER 3 provides some background on Category-Partition, the black-box testing technique we use, and machine learning. Our iterative approach is described in Figure 4. The results of two case studies are discussed in CHAPTER 5. Conclusions are drawn in CHAPTER 6. 


\section{CHAPTER 2 RELATED WORK}

In [8], the authors introduce a technique to learn a specification from execution traces. The generated specification is a non-deterministic finite automaton and the authors focus on mining uses of APIs or ADTs (i.e., their specification): nodes of the automaton are calls to the API (or ADT). The automaton is non-deterministic since not all behaviors are necessarily reverse-engineered (this depends on the test cases being used). The authors use a specific machine learning algorithm that generates a probabilistic finite state automaton (PFSA): probabilities are attached to transitions, specifying how often the transitions have been taken. The approach can be the starting point of a testing procedure: The (automaton) specification of an API can be learnt from its use in program $P_{1}$, and its use in program $P_{2}$ can be checked against the learnt specification. As opposed to our work, their approach does not guide the definition (or refinement) of test cases.

Execution traces are also used in [9] to learn program behavior: the authors record the execution of branches in the program (inter-procedural) control flow graph. The classifier being learnt is a map between execution traces (i.e., branch execution profiles) and behavior classes (e.g., fail/pass), and can be used to guide the construction (or extension) of a test suite. Assuming a test suite TS for program $\mathrm{P}$ exists and has to be augmented, $\mathrm{P}$ is first instrumented (to capture branch executions). The instrumented $\mathrm{P}$ is executed on TS and branch execution profiles are used to create a classifier. When a new test case is created, it is classified by the classifier. If the classifier cannot classify it, i.e., it cannot decide to which behavior class (e.g., 
fail/pass) the test case corresponds, the test case is associated with a class (i.e., the tester provides a behavior class, typically by building an oracle) and used to refine the classifier. This means that the test case triggers a behavior that was not recognized by the classifier. If the test case is recognized by the classifier, i.e., the behavior was already exercised by TS, it is discarded. Although the classifier helps the tester identify (somewhat) redundant test cases, thus avoiding the cost of building an oracle for them (an oracle is only built for unclassified test cases), the technique requires that the test case be defined by other means and executed before determining whether it is a useful addition or not. The strategy can only be practical if the tester can ensure that the new test data have high chances of triggering new behavior (otherwise, building test cases and more so executing them may be expensive), e.g., if an automatic test data generator is used, as suggested by the authors, there is a risk that many executed test cases be redundant and recognized by the classifier, therefore providing little help. Though the approach is very effective at determining whether a new test case is a useful addition, it provides little help for the definition of new, interesting test cases (the authors assume an automated test input and test case generation procedure is available).

Another execution trace based approach to test suite evaluation and extension is proposed in [10]. As long as the reverse-engineered specification, in the form of socalled 'likely invariants' [11], does not change when adding a test case to a test suite, the test case is deemed redundant. This approach does not require the construction of oracles, as opposed to the previous ones. The quality of the result however depends on the program invariant patterns that are used. The program invariants being 
discovered from execution information must be instances of a set of pre-defined invariant patterns. An invariant that does not fall into this category cannot be recognized. Furthermore, the approach does not provide guidance regarding the definition of new test cases.

This approach is expanded upon in [12] where the authors use a specification (invariants), reverse-engineered from passing test cases, to determine whether new automatically-generated (mainly randomly) test inputs are illegal, legal, and fault revealing based on the run-time monitoring of invariants. Illegal test cases violate the reverse-engineered pre and post conditions of a method, whereas fault revealing test cases only violate post conditions. However, such a test case may not necessarily represent a failure since the pre and post conditions may not be complete (they have been learnt from a set of test cases that may not have exercised every possible behavior). This is further expanded in [13] where reverse-engineered likely invariants [11] are analyzed by a constraint solver to automatically produce test inputs and test cases. Constraint solving is however limited (e.g., integer variables are supported but not floating point variables). In both [13] and [12], only likely invariants matching pre-defined patterns are considered (recall the discussion above).

In [14] the authors investigate how profiling deployed software, i.e., collecting execution information in the field, can help improve a test suite. No learning mechanism is employed though: additions to the initial in-house test suite entail repeating scenarios observed in the field and new test cases derived from trace data. The use of learning algorithms to understand program executions is applied to the problem of profiling deployed software in [15]. The objective is to be able to 
accurately classify program executions as fail or pass executions based on low-cost, limited instrumentation of deployed programs, rather than to improve a test suite.

Baudry et al. [16] discuss a white box technique to improve a test suite with a different aim: obtaining a test suite that will facilitate diagnosability by pinpointing faulty statements with a high accuracy. The proposed approach relies on a new testing criterion, that evaluates the "fault locating power" of a test case (measured using Tarantula ${ }^{1}[17]$ ), to evaluate the test suite to improve, and on a bacterial algorithm (an adaptation of a genetic algorithm [18]) to actually find improved test cases.

In [19] the authors present an adaptive sampling mechanism to identify feasible paths in a control flow graph with high traversing probability. Instead of uniformly sampling the set of paths in the control flow graph of a program (which usually contains a very large number of unfeasible paths), the authors devise an adaptive (learning) approach to ease the identification of feasible paths. The adaptive aspect of the approach is to learn, from the structure of known feasible paths, which branches to traverse in order to obtain a new feasible path.

In [20] the authors present a technique to improve a test suite based on a $\mathrm{Z}$ specification of the program/function under test and the Classification Tree method

\footnotetext{
${ }^{1}$ Tarantula is a visualization tool that gives the user a number of options for visualizing the testing results and the program itself. Tarantula uses the proportion of test cases that fail when executing a specific statement to determine the ranking of statements in terms of their likelihood to contain a fault. One important issue is that this assumes that test cases fail due to the same fault(s), a situation which nearly never occurs in the presence of multiple faults.
} 
$[21]^{2}$. They show how a classification tree of the program input parameters can be created semi-automatically from its $\mathrm{Z}$ specification. The classification can be enriched with any category/choice if this is deemed necessary by the tester. The Classification Tree method [21] can then be applied on the classification tree to build test cases. These test cases are predicates on the program input parameters, i.e., conjunctions of the categories/choices in the tree. These predicates are then combined with the predicates of the $Z$ specification (see [20] for details on the combination procedure) to: (1) determine the expected output of a test case, (2) refine the user defined test cases, completing their definitions with categories/choices that have been omitted when applying the Classification Tree method, and (3) identify problems in the $Z$ specification, such as incompleteness (though no guidance is provided to solve this issue).

Many other applications of machine learning techniques to software engineering exist in literature (e.g., [22, 23]) but are less related to our focus on test suite and test specification improvement.

To summarize, our approach differs from the above with respect to one or several of the following aspects: (1) It addresses black-box, functional testing, (2) It provides guidance in terms of new functional test cases to consider (and not only the

\footnotetext{
${ }^{2}$ The Classification Tree method [22] is a black-box partition testing technique, supported by a tool, similar to Category-Partition [6]. It allows a tester to define categories and choices (using the Category-Partition terminology) under the form of a tree and define test cases as combinations of classes (there is no notion of constraints like in Category-Partition though).
} 
improvement of existing ones), (3) It helps refine the test specifications from which test cases can be derived following a clear rationale. 


\section{CHAPTER 3 BACKGROUND}

Our approach, detailed in CHAPTER 4, relies on a well-known black box testing technique, namely Category Partition [6] (Section 3.1), as well as machine learning (Section 3.2).

\subsection{Using Category Partition}

Category Partition [6] is a well-known black-box testing technique. Compared to equivalence class partitioning that only partitions the input value domain and other black box testing techniques, the Category-Partition method is more general and encompassing. In Equivalence Class Partitioning, classes of input conditions called equivalence classes are identified such that each member of the class causes the same kind of processing and output to occur. In this method, the tester identifies various equivalence classes for partitioning. A class is a set of input conditions that are likely to be handled the same way by the system. If the system were to handle one case in the class erroneously, it would handle all cases erroneously. The Category-Partition method generates test cases that cover functions. To apply the Cateory-Partition method, one identifies the parameters of each function, as well as external (environment) conditions that affect the function's execution behavior. Then one identifies the characteristics (referred to as categories) of each parameter (and environment conditions) and the choices of each category. Categories are properties of parameters (and environment conditions) that can have an influence on the behavior of the function under test (e.g., comparing the lengths of two sides in the 
Triangle program example [2]). Choices (e.g., whether two sides of the triangle are equal or not) are the potential values of a category which stands for a certain character of the category. Choices for a category are mutually exclusive, i.e. for each category only one choice is feasible. Test frames (abstract test data) and test data are generated according to the identified categories and choices: different criteria exist for combining choices in test frames [6].

To illustrate how the Category Partition (CP) [6] black-box testing method works, let us take the well-known and simple Triangle program example [2], which we will also use as a working example to illustrate our method: The complete application of $\mathrm{CP}$ to the Triangle program is available in Figure 1 . The test input values of the triangle program characterize the length of triangle sides $(a, b, c)$ and its output determines whether these sides correspond to an equilateral, isosceles, or irregular triangle. In addition, the program may determine that the sides cannot correspond to a triangle (based on checking certain inequalities, i.e., choices $\mathrm{C} 6, \mathrm{C} 12$, and $\mathrm{C} 18$ in Figure 1) or the side values are illegal (choices $\mathrm{C} 2, \mathrm{c} 8$, and $\mathrm{C} 14$ in Figure 1).

In addition to categories and choices describing input parameters of the program, $\mathrm{CP}$ requires the identification of categories and choices for environment conditions, i.e., conditions of the environment of the program that may affect its behavior (e.g., contents of a database, state of external systems, load of the processor or network). $\mathrm{CP}$ can therefore help characterize functional as well as non-functional behavior, targeting functional testing, performance testing, and robustness testing. 
In our context, once categories and choices are defined, we use them to automatically transform each test case into an "abstract" test case. (There are as many abstract test cases as test cases.) In our Triangle example, these properties may correspond to Boolean expressions stating relationships between sides, e.g., how a compares to $\mathrm{b}$ and c (category 3 in Figure 1). Taking the "a compared to b and c" category as an example, choices could correspond to the two mutually exclusive situations where $a<=b+c$ (choice c5) and $a>b+c$ (choice c6). Choices $c 2 \quad(a<=0), c 8 \quad(b<=0)$, and C14 $(\mathrm{c}<=0)$ are error conditions, i.e. the length of triangle side cannot be less than or equal to zero. In addition, though we do not make use of them in our approach, Category-Partition requires that "properties" and "selectors" be defined to model interdependencies between choices and thus be used to automatically identify unfeasible combinations of choices across categories [6].

\begin{tabular}{|c|c|}
\hline 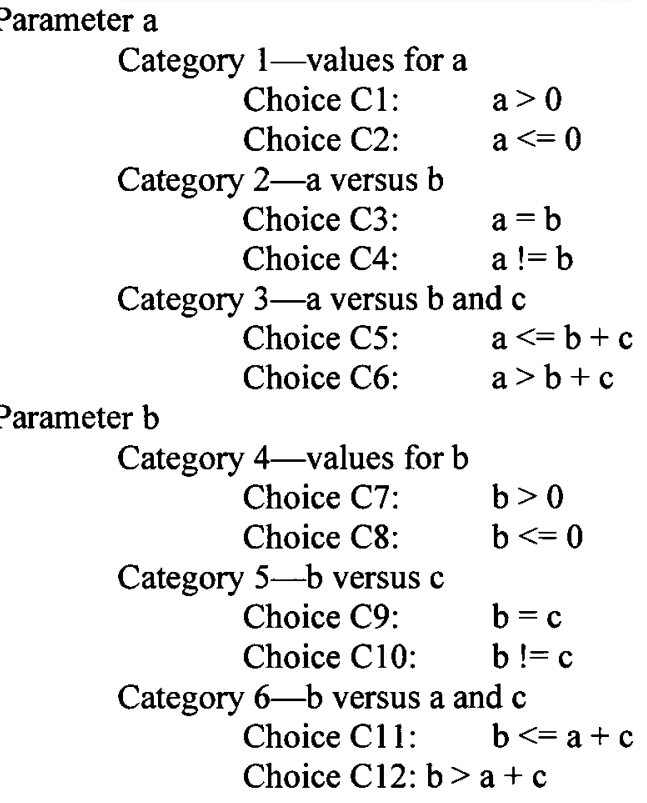 & 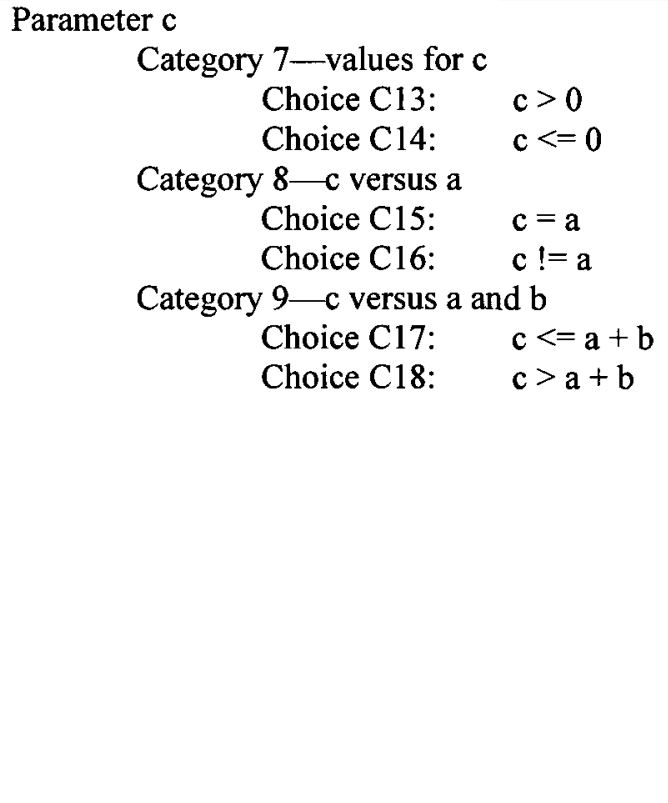 \\
\hline
\end{tabular}

Figure 1 Triangle Program CP Specification 


\subsection{C4.5 Decision Trees (DT)}

There is a large number of machine learning and data mining techniques [24]. They differ widely in terms of their basic principles, their working assumptions, and their weaknesses and strengths. None of the techniques is inherently better than the other and which one is most appropriate tends to be context dependent. Some of these techniques focus on classification, which is the problem at hand in this thesis as we want to learn about the relationship between inputs and environmental properties (categories and choices) and output equivalent classes.

A specific category of machine learning techniques focuses on generating classification rules [24] which are easily amenable to interpretation. Examples of such techniques include the C4.5 decision tree algorithm [7] (where the paths from the root node of the tree to any leaf can be considered a rule) or the Ripper rule induction algorithm [25]. In our context, the rules would look like properties on test inputs that are being associated with outputs. The main advantage of these techniques is the interpretability of their models: certain conditions imply a certain output equivalence class.

Some techniques, like C4.5, partition the data set (e.g., the set of test cases) in a stepwise manner using complex algorithms and heuristics to avoid over-fitting the data (i.e., determining how deeply to grow a decision tree), with the goal of generating models that are as simple as possible. Others, like Ripper, are so-called covering algorithms that generate rules in a stepwise manner, removing observations that are "covered" by the rule at each step so that the next step works on a reduced set 
of observations. With coverage algorithms, rules are interdependent in the sense that they form a "decision list" where rules are supposed to be applicable in the order they were generated. Because this makes their interpretation more difficult, we will use a classification tree algorithm, namely C4.5, and use the WEKA tool [24] to build and assess the trees.

To illustrate the notion of $\mathrm{C} 4.5$ decision tree and the output generated by Weka, we take the weather problem [24] as an example. The weather problem is a tiny dataset that relates the weather conditions that are suitable for playing some outdoor game. Instances in the dataset are characterized by the values of four attributes that measure different aspects of weather conditions: Outlook, temperature, humidity, and windy; and the outcome is whether to play or not to play. Table 1 shows the dataset of 14 instances that is used in the weather problem with four attributes and their values; the last column indicates the output. Outlook can be sunny, overcast, or rainy; Temperature can be hot, mild, or cool; humidity can be high or normal; and windy can be true or false.

\begin{tabular}{|l|l|l|l|l|}
\hline Outlook & Temperature & Humidity & Windy & Play \\
\hline Sunny & Hot & High & False & No \\
\hline Sunny & Hot & High & True & No \\
\hline Overcast & Hot & High & false & Yes \\
\hline Rainy & Mild & High & False & yes \\
\hline Rainy & Cool & Normal & False & Yes \\
\hline Rainy & Cool & Normal & True & No \\
\hline Overcast & Cool & Normal & True & Yes \\
\hline Sunny & Mild & High & False & No \\
\hline Sunny & Cool & Normal & False & Yes \\
\hline Rainy & Mild & Normal & False & Yes \\
\hline Sunny & Mild & Normal & True & Yes \\
\hline Overcast & Mild & High & True & Yes \\
\hline Overcast & Hot & Normal & False & Yes \\
\hline Rainy & Mild & High & True & No \\
\hline
\end{tabular}

Table 1 The Weather Data [24] 
Based on the data in Table 1, WEKA returns ${ }^{3}$ the result of Figure 2, applying the C4.5 algorithm. Figure 2 shows a decision tree (lines numbered 1 to 7 ), reports on the number of leaves in the tree (line 8), and shows a confusion matrix (bottom of Figure).

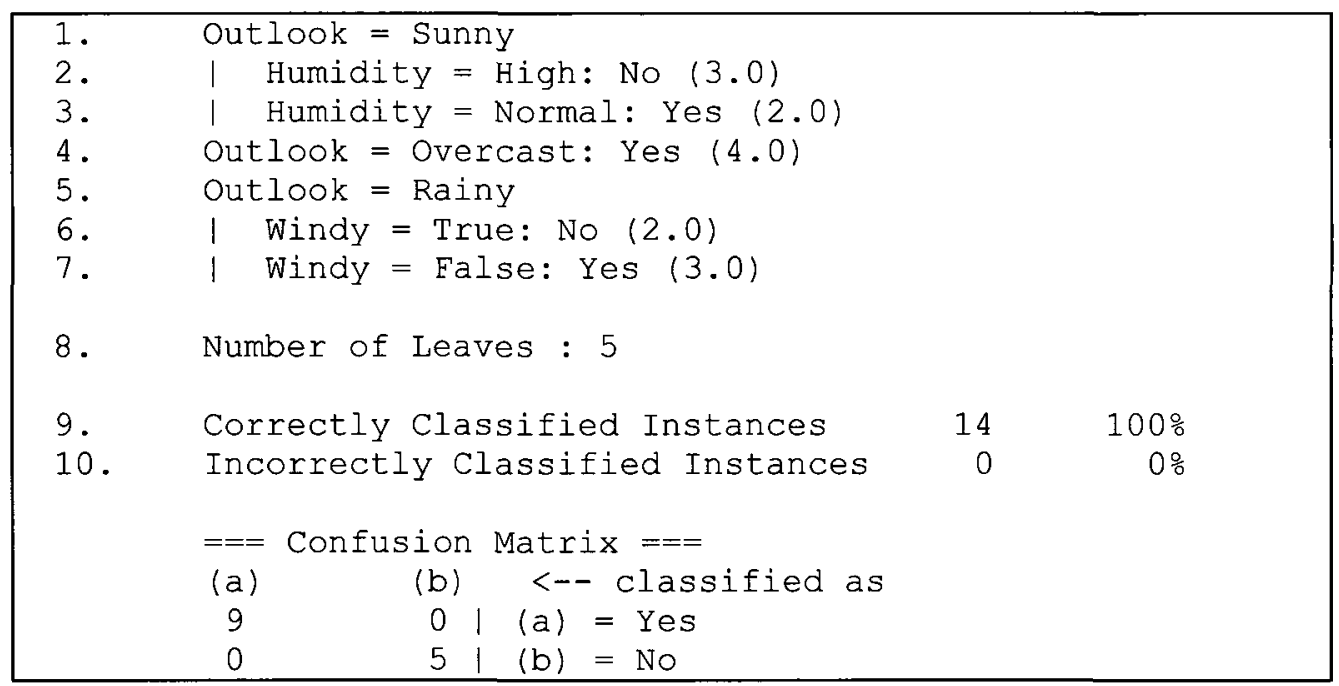

Figure 2 C4.5 decision tree for the weather data

It is easy to read a set of rules directly off a decision tree. Traversing a decision tree from the root to each leaf gives a rule, with the path conditions as the antecedent and the leaf as the class or output. This produces rules that are unambiguous, i.e. the order of the conditions does not matter. In this example, the root is the attribute outlook that has three possible values: Sunny, Overcast, and Rainy. There are two sub-trees: a Humidity sub-tree below Sunny (lines 1 to 3 ), and a windy sub-tree below Rainy (lines 5 to 7). There is a total of 5 rules, i.e., 5 leaves, that classify all the instances in Table 1.

\footnotetext{
${ }^{3}$ Using the default verbosity level of WEKA.
} 
It is now easy to read the rules, as illustrated in Figure 3. For instance, rule 1 (lines 1 and 2 in Figure 2) can be read as: If Outlook is Sunny and Humidity is High then the output is don't play. The number in brackets following each leaf indicates the number of instances in the input set that are classified by each rule. This number may be followed by a second number (which is not the case in Figure 2), e.g., 3.0/1.0, in which case the second value (1.0) indicates the number of classification errors (or the number of instances misclassified by that rule) encountered out of the total number of classifications made from the training data in that particular path of the decision tree (3.0). C4.5 builds decision trees from a set of training data using the concept of information entropy [24]. At each node of the tree, C4.5 chooses one attribute of the data that most effectively splits its set of samples into subsets enriched in one class or the other. Its criterion is the normalized information gain (difference in entropy) that results from choosing an attribute for splitting the data. The $\mathrm{C} 4.5$ algorithm then recurses on the smaller sublists. Lack of the training data may result in a tree where some leaves (rules) may have no instances covered.

Lines 9 and 10 in Figure 2 show the number (and percentage) of correctly and incorrectly classified instances respectively.

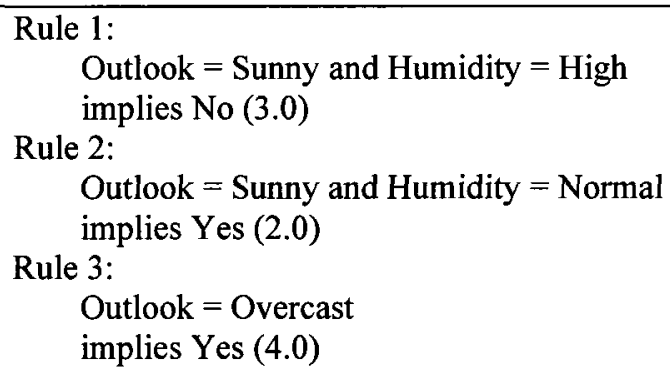

Figure 3 Rules explained (from Figure 2) 
A confusion matrix [26] contains information about actual and predicted classifications done by a classification system. The performance of such systems is commonly evaluated using the data in the matrix. From the confusion matrix in Figure 2 we see that, nine instances of the class yes are correctly classified using the generated rules. Five instances of the class No are correctly classified using the generated rules. Zero instances were incorrectly classified.

Let us suppose that in the weather problem two instances of class yes are misclassified as No. The confusion matrix would look like:

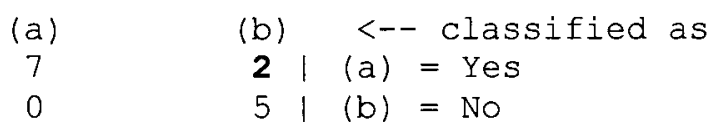

This matrix shows that 7 instances of the known class Yes (Play) were correctly classified using the generated rules as members of class Yes. 5 instances of the known class No (Don't Play) were correctly classified using the generated rules as members of class No. 2 instances were incorrectly classified. 


\section{CHAPTER 4 METHOD}

An overview of the steps involved in the MELBA (MachinE Learning based refinement of BlAck-box test specification) method is briefly described in Section 4.1. Section 4.1 also illustrates how an abstract test suite is obtained and why it is important to have the abstract test suite as opposed to the raw test data. Our method takes as inputs the test suite, and test specification following the Category-Partition (CP) strategy, to be re-engineered. Based on the Category-Partition specification, test cases are transformed into abstract test cases which are tuples of pairs (category, choice) associated with an output equivalence class (instead of raw inputs/outputs). A machine learning algorithm ( $\mathrm{C} 4.5$ decision tree algorithm) is used to learn rules that relate pairs (category, choice), modeling input properties, to output equivalence classes. These rules are then analyzed to determine potential improvements to the test suite (e.g., redundant test cases, need for additional test cases) as well as improvements to the Category-Partition specification (e.g., need to add a category or choices). Our approach is to identify problems in results produced by $\mathrm{C} 4.5$ (Section 4.2) and to relate them to potential causes in terms of test suite or test specification deficiencies (Section 4.3). We illustrate these two steps on our Triangle working example (Figure 1). We discuss strategies to improve a test suite in Section 4.4.

\subsection{Overview}

Figure 4 provides an overview (as a UML activity diagram) of the steps involved in the MELBA (MachinE Learning based refinement of BlAck-box test specification) 
method we will describe in detail in the next sections. The inputs of the method are the test suite to be re-engineered and a test specification (left side of Figure 4).

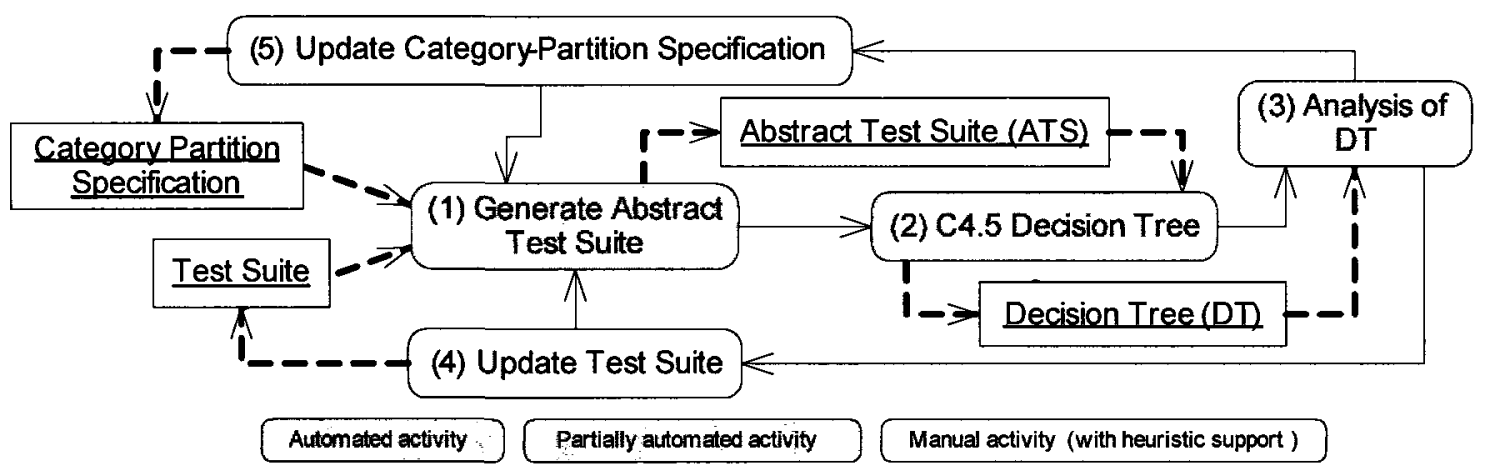

Figure 4 The MELBA Method

\subsubsection{An Iterative Process}

We do not make any specific assumption regarding the contents of a test case and the software unit under test (SUT), other than the feasibility of transforming test cases into abstract test cases given pre-defined categories and choices. In particular, we do not assume that the test suite has been originally derived according to the CategoryPartition method. Though the test specification is assumed in this thesis to follow the category-partition [6] (CP) strategy, future work will investigate how our method could be tailored to other black-box strategies. In the context of CP, the SUT is typically decomposed into user functionalities (e.g., use case) which are then independently tested. However, CP can be applied to the test of sub-systems or even methods, and as other black-box test techniques, the complexity of testing depends on the behavioral specification of the SUT, not necessarily its source code size.

In practice, this test specification may or may not exist to start with, especially if no black-box strategy was used to identify the test cases. In the latter case, which is 
likely to be the most common situation, the test specification has to be reverseengineered, to some extent or created from high level (likely plain language) specification. Furthermore, the output domain has to be divided into equivalence classes. In the context of Category-Partition, the Triangle program [2] is so simple that its output is already under the form of equivalence classes: equilateral, isosceles, irregular triangles, and not a triangle. However, even this could be simplified, for example, into two equivalence classes: Triangle, not Triangle. The level of granularity of this partition of the output domain is a decision of the tester. Increased granularity will result into increased testing effort but will characterize the SUT behavior in a more precise way

As the input domain is modeled using CP categories and choices (Section 3.1), the test suite is then transformed into an abstract test suite (Activity 1 in Figure 4). Recall that each test case in the test suite is transformed into one abstract test case, and that therefore the abstract test suite has the same number of elements (size) as the test suite. An abstract test case shows an output equivalence class and pairs (category, choice) that characterize its inputs, instead of raw inputs. Once the Category-Partition specification is defined, this abstraction activity is easy to automate, as it was done in our case studies. The definition of categories and choices, on the other hand, requires much thinking as one must identify categories and define choices so that they determine output equivalence classes. This represents an up-front investment, and is an issue further discussed in section 4.1.2. Note that the initial categories and choices defined by the tester do not have to be perfect as our method will help identify 
problems in their definitions. Additionally, the categories and choices can be defined at varying levels of granularity, which is a decision resting with the tester.

Once an abstract test suite is available, a machine learning algorithm (C4.5 [7]) is used to learn rules that relate pairs (category, choice), modeling input properties, to output equivalence classes (Activity 2 in Figure 4). An example of such rule was discussed in Section 3.2.

These rules are in turn analyzed by a tester (Activity 3 in Figure 4) to determine potential problems that may indicate redundancy among test cases or the need for additional test cases (Activity 4 in Figure 4). Those rules may also indicate that the Category-Partition specification needs to be improved: e.g., an important category is missing or certain choices are ill-defined (Activity 5 in Figure 4). In the next sections, we will detail a number of heuristics that can be used to automatically analyze the C4.5 rules and investigate ways to improve test suites and CP specifications.

The improvement process in Figure 4 is iterative as improvements to either the test suite or test specification can lead to the identification of new problems to be addressed. The learning algorithm will therefore be repeatedly executed (edges from Activities 4 and 5 to Activity 1, followed by Activity 2), which is not an issue as obtaining C4.5 decision trees for a few thousands of (abstract) test cases and a few dozen categories is quick (see Section 4.1.2). The improvement process stops when no more problems can be found in the rules learnt by the machine learning algorithm (Activity 3). 
One issue is the presence of faults in the software under test and its impact on MELBA and the C4.5 learning algorithm. MELBA assumes that the initial test suite has been run, failures have been detected and the corresponding faults corrected. In short, the starting point of the iterative improvement process is a possibly incomplete but passing set of test cases. However, as the test suite is augmented with new test cases, failures can arise and new faults can be detected. These faults must then be corrected and the new test cases must pass before re-running $\mathrm{C} 4.5$ and obtain a new decision tree. Otherwise, since some of the outputs might be incorrect, this might lead to misclassifications in the tree which, though they would not necessarily prevent the use of MELBA, could make the decision tree analysis more complex.

In the case of forward development, when test cases are defined, possibly even before any source code is written, the approach can still be applied: test inputs and corresponding (expected) outputs are used during the whole refinement process and this does not require any test case execution. When test case implementation is available and identifying the expected output is deemed too difficult (Oracle Problem [27]), test cases can be assembled and the actual output collected to be used in the refinement process.

\subsubsection{Manual Effort and Automation}

Once the CP specification is defined, the transformation of test cases into abstract test cases is easy to automate. For instance, in our first case study, using a CategoryPartition specification of 11 categories and 33 choices and a test suite of 221 test cases, it took a couple of seconds to create 221 abstract test cases. Using the more 
complex, second case study, namely the Space program, for which 83 categories and 582 choices are defined, 13,585 test cases are abstracted in less than a minute. In short, Activity 1 in Figure 4 is automated and fast.

The definition of categories and choices, on the other hand, requires much thinking as one must identify categories and define choices so that they determine output equivalence classes. This requires an understanding of the system domain but is, on the other hand, what a tester would typically do when trying to reengineer a test suite, for instance using $\mathrm{CP}$ or any other black-box test technique. Though this represents an up-front investment, there is no way one can reuse a test suite or modify a system with confidence without making an effort to understand the relationships between the inputs and outputs of the system.

Activity 2 is another automated step, for which we use the WEKA tool, which implements C4.5. For our first case study, it took less that a second for WEKA to generate a decision tree. In the case of the larger Space problem (second case study), it took eight seconds to generate a tree based on 13,585 abstract test cases.

Activity 3 is partially automated. On the one hand, much information is automatically provided in the WEKA output: misclassifications, categories and choices used in learnt rules, number of instances (i.e., abstract test cases) involved in rules. This information is the source of our heuristics for problem identification described in Section 4.2. The tester then has to identify the causes of those problems, a process that we support with a set of guidelines (Section 4.3). 
Activities 4 and 5 are not automated at this point, as this relies on the know-how and expertise of the tester. However, as discussed in the next section, we provide guidance to help identify which categories/choices need to be refined, which abstract test cases need to be defined. Test suite amendment (Activity 4) requires some effort but this is an effort that is anyway incurred if one is trying to improve a test suite.

\subsubsection{From raw test data to abstract test cases}

The rules produced by $\mathrm{C} 4.5$ can be seen as tuples of choices associated with an output equivalence class (or output equivalence class value). Using the triangle $\mathrm{CP}$ in Figure 1 , the test case $(a=2, b=3, c=3)$ could be abstracted into a tuple such as $(a<=b+c$, $b=c$, Isosceles), where: $a<=b+c$ is a choice for category Category $3-a$ versus $b$ and $c$ in Figure 1; b=c is a choice for category Category $5-b$ versus $c$ in Figure 1. Note that the tuple would contain pairs of the form (category, choice) but we only show choices here to simplify the discussion. Notice that tuples typically involve many more choices as every existing choice condition that applies to a test case is used when creating the corresponding abstract test case. The test case above could then be abstracted into $(a>=0, a !=b, a<=b+c, b>0, b=c, b<a+c, c>0, c !=a$, $\mathrm{c}<=\mathrm{a}+\mathrm{b}$, Isosceles). Last, it may happen that none of the choices defined for a specific category can be used when creating an abstract test case ${ }^{4}$. In such a situation, we add a "not applicable" (or N/A) choice to the category and use this pseudo choice

\footnotetext{
${ }^{4}$ This is typically the case when choices cannot be combined across categories, or when categories cannot be combined. Such a situation would be specified with "properties" and "selectors" if we were applying $\mathrm{CP}$ for the purpose of defining test cases, instead of using $\mathrm{CP}$ to characterize existing test cases.
} 
in the tuple. For example, assume a program manipulates a string of characters and its behavior depends on whether the string contains only numbers or only letters (the behavior would furthermore depend on whether the string contains capital letters or not). Then one would define (at least) a category Cat1 with two choices ( 1 1 and $\mathrm{C} 2$, respectively) for the two different types of strings, and a category cat2 for strings containing letters with two choices ( $\mathrm{c} 3$ and $\mathrm{C} 4$, respectively) specifying whether the string contains capital letters or not. Suppose now that we want to create the abstract test case for a test case where the input parameter contains only numbers. Choice $\mathrm{C} 1$ would be used in the tuple but none of the choices of cat 2 can be used. We then define a N/A choice for Cat2 and use it in the tuple.

We need to create abstract test cases from concrete (raw) test cases since using raw data will generate meaningless and possibly incorrect decision trees. Rules generated from raw data are meaningless and possibly incorrect because the machine learning algorithm cannot learn which properties are of interest: since the raw data do not contain any information on those properties, the rules can only relate values of the parameters instead of properties of those parameters. Eventhough, in C4.5 continuesvalued attributes are incorporated into the learned tree by dynamically defining new discrete-valued attributes that partition the continuous attribute value int o a discrete set of intervals, the only question is how to select the best interval. There are other methods for dealing with numeric attributes (see [40]), but mainly algorithms that can deal with them look to discretise them in some way. This means that they can then be treated just as categorical attributes. But instead of using the dynamically categored values, we generate such intervals by using $\mathrm{CP}$ which can then be improved through 
the Melba method. If raw data were to be used, they are to be well distributed in the domain space; the generated tree would then be meaningful and easy to understand. This is however unlikely to happen in pratice. To fascilitate the learning process, further guidance needs to be provided to the learning algorithm to generate a meaningful tree. Let us use the Triangle program as an example to illustrate this. Executing $\mathrm{C} 4.5$ on the raw data for the Triangle program, i.e., the nine test cases in Appendix A.1.4 (Table 4), we obtain the decision tree of Figure 5.

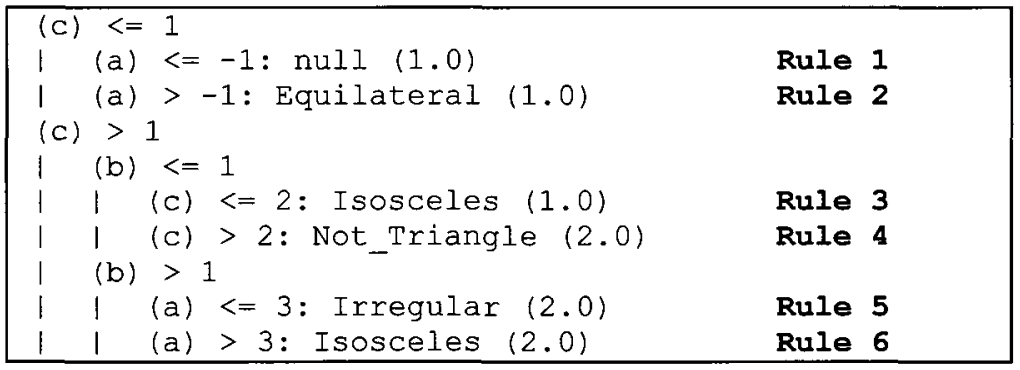

Figure 5 Triangle Tree generated with raw data

Rule 2 in Figure 5 for instance indicates that if $c<=1$ and $a>-1$ then the output class is Equilateral. This is not correct since $c=0$ and $a=0$, which satisfy the condition, should not correspond to a valid triangle. As a second example, consider Rule 6. It indicates that if $c>1$ and $a>3$ then the triangle is isosceles. Again, this is not correct as other conditions should hold to have an isosceles triangle. Therefore, we need to transform the (raw) test cases into abstract test cases to guide the learning algorithm. We use the Category-Partition (CP) method to define the test specification and then use the categories and choices to do the mapping between the raw test case data and the categories and choices of the CP to obtain an abstract test suite (as described earlier). 
Let us consider the Triangle program with the same 9 test cases. However, this time, instead of using the raw data, we use the abstract test cases obtained using the $\mathrm{CP}$ specification in Appendix A.1.2: Table 5 in Appendix A.1.4. We obtain the decision tree of Figure 6. The tree clearly shows the conditions under which each type of triangle is obtained. The rules are accurate and meaningful.

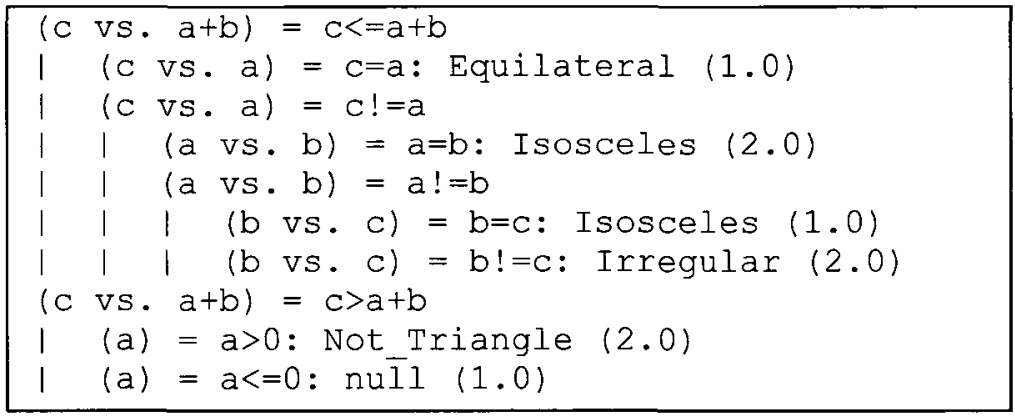

Figure 6 Triangle Tree generated with abstract test data

\subsection{Identifying problems in $\mathbf{C} 4.5$ decision trees}

When analyzing a C4.5 decision tree in the context of our method, we can identify a number of potential problems:

Case 1-Instances (abstract test cases) can be misclassified: the wrong output equivalence class is associated to a test case. In other words, a test case belongs to a tree leaf where the majority of instances belong to another output equivalence class.

Case 2-Certain categories or choices are not used in the tree (i.e., they are not selected as attribute to split a (sub) set of instances in the tree).

Case 3-Certain combinations of choices, across categories, are not present on any path, from the root node to any leaf of the tree. 
Case 4-A leaf of a tree contains a large number of instances (test cases).

As mentioned previously, all of the above cases can be automatically detected by a dedicated tool. However, as discussed next (Section 4.3), determining the exact cause of the problem can only be facilitated but not entirely automated.

Note that cases 2 and 3 above have been shown to be the main issues when practitioners apply Category-Partition [6]. In [6] the authors report on three empirical studies during which they studied the ease with which subjects apply the CategoryPartition method. Their observation (conclusion) is that practitioners need guidance since missing categories, missing choices, and ill-defined choices (e.g., non disjoint choices) often occur when applying Category-Partition. The authors suggest that practitioners follow a checklist to systematically identify these problems. In some way, we provide automated support and a set of heuristics to help address these problems. Our work also goes beyond this as we also address the improvement of the test suite.

\subsection{Linking problems to causes}

The problems discussed above all have one or more potential causes, as summarized in Figure 7. Misclassifications in the decision tree (Case 1) can have two potential causes: missing category or choice (case 1.1, Section 4.3.1), ill-defined choices (case 1.2, Section 4.3.2). Some categories (or choices) can be defined in the $\mathrm{CP}$ specification but not end up being used in the decision tree (Case 2). This can also be explained by several potential causes: useless category or choice (case 2.1 , Section 4.3.3), missing test cases (case 2.2, Section 4.3.4), and ill-defined choices (case 1.2, 
Section 4.3.2). Even if all expected categories show up in the tree, certain combinations of choices across categories may not be exercised by any branch in the tree (Case 3). This may be the result of several potential causes: unfeasible combination (case 3.1, Section 4.3.5), missing test cases (case 2.2, Section 4.3.4). The last problem we cover is related to too many test cases in a rule (Case 4). It is in general important to minimize functional test suites and ad hoc test suites often turn out to contain such redundancy. In our context, when many test cases end up in a decision tree leaf then the question arises whether they are all necessary. Indeed, this means that a number of test cases exercise the same choice combination for a subset of categories and then, as a result, fall in the same output equivalence class. The tester may then consider whether all these test cases in the same tree leaf are necessary as they have similar properties, lead to similar outputs, and probably exercise the software in a similar fashion. Though this remains a subjective decision that only the tester can make, the decision tree points out potential redundancy. There may be, however, two reasons for redundancy: redundant test cases (case 4.1, Section 4.3.6), ill-defined choices (case 1.2, Section 4.3.2).

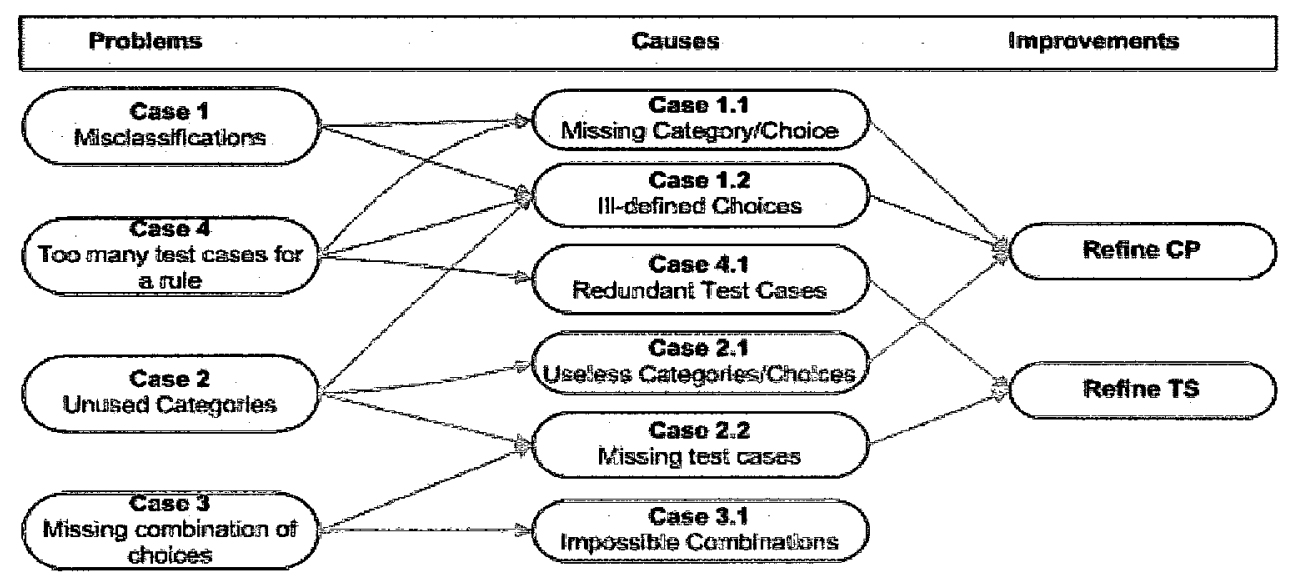

Figure 7 Problems and potential causes 
Improvements to $\mathrm{CP}$ depend on cause(s) and on context. This is therefore up to the tester. Improvements to the test suite (TS) can be made following our heuristic discussed in section 4.4. As illustrated in Figure 7 both cases 1.2 and 2.1 (sections 4.3.2 and 4.3.3) will lead to the refinement of the $\mathrm{CP}$ specification, either by adding/deleting categories/choices or redefining choices for existing categories. Section 4.3 .2 would require the modification of choice definitions, possibly leading to an increased number of test cases. Section 4.3.3 may lead to removing a category from the $\mathrm{CP}$ specification, thus leading to a smaller number of test frames and therefore fewer test cases. As illustrated in Figure 7, cases 1.1, 4.1, and 2.2 (sections 4.3.1, 4.3.6, and 4.3.4) will lead to the refinement of the test suite (TS). Case 2.2 (Section 4.3.4) requires the addition of test cases. It should be fairly easy to differentiate case 4.1 (section 4.3.6) from case 1.2 (section 4.3.2): The presence of misclassifications suggests that 4.3 .2 is more plausible; No misclassification probably indicates the presence of redundant test cases. Case 3.1 (section 4.3.5) clearly does not result in any modification of CP or TS (Figure 7).

\subsubsection{Case 1.1 - Missing category/choice}

A category or choice is missing although it is necessary to determine the appropriate output equivalence class.

Example 1 in Figure 8 is produced by $\mathrm{C} 4.5$ if one omits category 7 when using $\mathrm{CP}$ for the Triangle program (Figure 1): category 7 tests whether $\mathrm{c}$ is strictly positive or not (it has two choices). This omission results in the rule of Example 1 to show the misclassification of 2 instances (abstract test cases) among 26 instances (24+2), 
classified as Isosceles triangles by the rule. Recall section 3.2 that decision trees splits on attributes (i.e category/choices) to generate the DT. If the C4.5 algorithm does not find an attribute that correctly classifies instances, it will use the next best attribute to split on, eventhough it may result in misclassification. The confusion matrix in Figure 9 tells us that those two instances belong to the Not_Triangle class: 2 instances (in bold face) of the class Not_Triangle are misclassified as Isosceles.

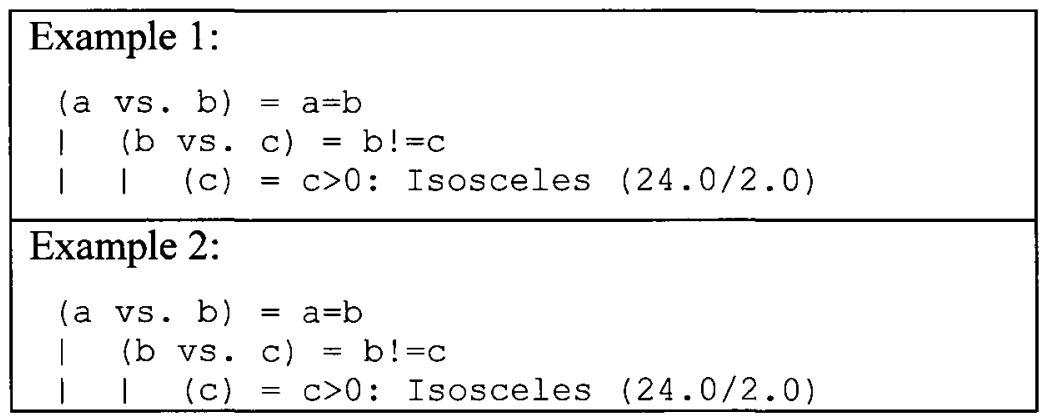

Figure 8 Examples using the Triangle Program

\begin{tabular}{|rrrrrr|c|}
\hline$===$ & Confusion & Matrix & $===$ \\
$\mathrm{a}$ & $\mathrm{b}$ & $\mathrm{c}$ & $\mathrm{d}$ & $\mathrm{e}$ & $<--$ & classified as \\
54 & 0 & 0 & 0 & 0 & $\mathrm{a}$ & $=$ Equilateral \\
0 & 66 & 0 & 0 & 0 & $\mathrm{~b}=$ Isosceles \\
0 & 0 & 60 & 0 & 0 & $\mathrm{c}=$ Irregular \\
0 & 2 & 18 & 40 & 0 & $\mathrm{~d}=$ Not Triangle \\
0 & 0 & 0 & 0 & 12 & $\mathrm{e}=$ nul \\
\hline
\end{tabular}

Figure 9 Confusion matrix for the Triangle Program with Category 9 removed

\subsubsection{Case 1.2 - Ill-defined choices}

Even though a category may be necessary and present in the characterization of test cases, the choices may be ill-defined, making the category a poor attribute to explain the output equivalence classes.

Assuming choices (C17 and C18) of category 9 (Figure 1), which is to compare length $c$ to lengths $a$ and $b$, are incorrectly specified as follows: 


$$
\begin{array}{ll}
\text { C17: } c<a+b & \text { (should be }<=\text { ) } \\
\text { C18: } c>=a+b & \text { (should be }>\text { ) }
\end{array}
$$

C4.5 returns the rule in Example 2 of Figure 8, showing two misclassified instances. Because the relational operators were changed (by moving the " $="$ operator from $c 17$ to $c 18$ ), these misclassifications are due to abstract test cases where $c=a+b$. Similarly, ill-defined choices may make a category irrelevant as it does not accurately determine the output classes anymore. Additionally, ill-defined choices can lead to misclassifications but also to the impossibility for $\mathrm{C} 4.5$ to further generate accurate rules for a large number of instances.

\subsubsection{Case 2.1 - Useless category or choice}

A category may be irrelevant if it turns out not to play a role in determining output equivalence classes. This may be due to the fact that the defined output classes are too rough for the category to play a role or simply that it is redundant (correlated) with other categories.

For example, the following category, if added, obviously will not play a role in determining the type of a triangle formed by sides $a, b$, and $c$, since its choices are redundant with the choices of categories 2,5 and 8 in the triangle $\mathrm{CP}$ specification (Figure 1). When applying CP, this category would not be selected by $\mathrm{C} 4.5$, and would therefore not appear in the decision tree.

CATEGORY 10 - $\mathrm{c}$ compared to a

C19: $\mathrm{c}>\mathrm{a}$

$\mathrm{C} 20: \mathrm{c}<=\mathrm{a}$ 


\subsubsection{Case 2.2 - Missing test cases}

Missing test cases can also lead to a category or choice not being selected. For example, there may not be test cases that exercise some or all of the choices of a category, thus resulting in that category being partly used (not all its choices are used) or not used at all in the decision tree. Recall section 3.2 that if there is not enough training data (i.e. test cases) that excercises all the variations of the program, the DT will have rules that are not covered by any test cases, and even combinations of choices that are not existent in the decision tree.

For example, to select an extreme case, if all test cases where $a<=0$ are removed from the test suite of the Triangle program (i.e., in all the test cases, a>0) then category 1 (Figure 1), which tests whether a is strictly positive or not, will not be selected as this category does not differentiate test cases (instances). Similarly, if test cases that exercise certain combinations of choices are missing from the test suite, then it is impossible for the tree to identify such combinations as relevant to determine output classes.

\subsubsection{Case 3.1 - Unfeasible combinations}

As it is often the case in the context of $\mathrm{CP}$, some combinations of choices may not be feasible (recall section 3.1).

For example, combination of choices $c 6$ (i.e., $a>b+c$ ) and $c 18$ (i.e., $c>a+b$ ) (Figure 1) is not feasible. Recall (Section 4.1.3) that when building an abstract test case from a 
concrete test case, we add a N/A choice when a category does not apply to a test case, therefore also suggesting an unfeasible combination of choices.

\subsubsection{Case 4.1 - Redundant Test Cases}

The most straightforward reason is of course the presence of redundant test cases, as described previously. The second reason for redundant test cases is the presence of ill-defined choices (case 1.2, Section 4.3.2). Ill-defined choices lead to misclassification and thus to a rule that covers many test cases (too many test cases that should be covered by a larger number of rules if choices were correctly defined).

For example the following rule covers 60 test cases (instances); the tester may consider whether all these test cases are necessary as they have similar properties and lead to similar output.

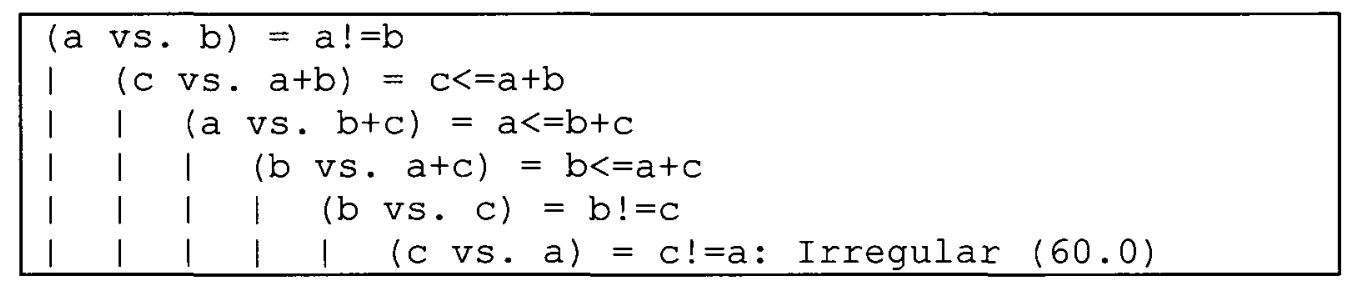

\subsection{Heuristics for Refining Test Suite}

As illustrated in Figure 7, a number of causes lead to updating the test suite, either by adding test cases to the test suite (section 4.4.1), or by removing test cases from the test suite (section 4.4.2). 


\subsubsection{Adding Test Cases}

As discussed previously, different reasons can lead to the addition of test cases: a choice may be missing in the tree; a category may be missing in the tree; certain choices are used in the tree but some of their combinations may be missing.

If a category (or choice) is not selected, and the category is deemed useful, then the tester has to create test cases involving each choice of the category ${ }^{5}$. However, the question is which combinations with other choices to include in the test suite? The first solution is to follow the CP method and build all the feasible (according to properties and selectors) combinations of choices and select the ones that are missing in the abstract test suite. We have however discussed that those properties and selectors were not required for applying our method (Section 3.1). Furthermore, this is an expensive option that does not make use of the information provided by the decision tree.

An alternative is to identify which combinations of choices may be relevant to determine the output class and could be missing from the test suite by using the decision tree. Assume that part of the tree obtained from C4.5 shows categories Cat1, Cat2, and Cat 3 with choices $\mathrm{C} 1$ and $\mathrm{C} 2, \mathrm{C} 3$ and $\mathrm{C} 4$, and $\mathrm{C} 5$ and $\mathrm{C} 6$, respectively, as illustrated in Figure 10 (a). Such a tree excerpt indicates that combining $\mathrm{c} 2$ of category Cat 1 with $\mathrm{C} 5$ or $\mathrm{C} 6$ of category Cat 3 plays a role in determining output

\footnotetext{
${ }^{5}$ As a special case, we consider the situation where the tree shows a possible rule (i.e., possible choice combination) with no instance. The tester can then simply add a test case for that rule satisfying the corresponding choice combination (if that combination is feasible).
} 
equivalence classes (the pairs of choices belong to different paths in the tree). Since Cat1 has another choice than $\mathrm{C} 2$, namely $\mathrm{C} 1$, we may reasonably expect that Cat 3 will also be relevant to determine the output in the context of $\mathrm{c} 1$ and that the tester should therefore combine choice C1 with Cat3's choices. Similarly, the tree suggests that the combinations of $\mathrm{C} 2$ with Cat2's choices may be missing in the test suite, thus resulting in four test cases being added ${ }^{6}$.

This heuristic can be generalized to cases where category cat1 is not a parent of Cat 2 and Cat 3 in the tree but rather an ancestor of cat 2 and cat 3 (i.e., there are intermediate categories): Figure 10 (b).

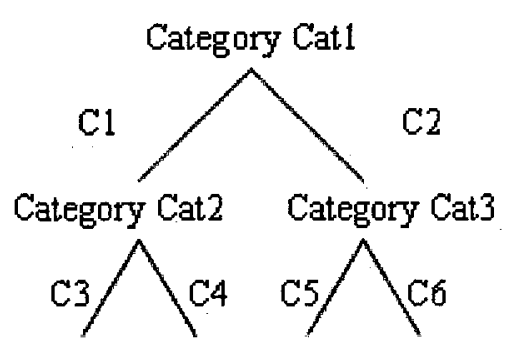

(a)

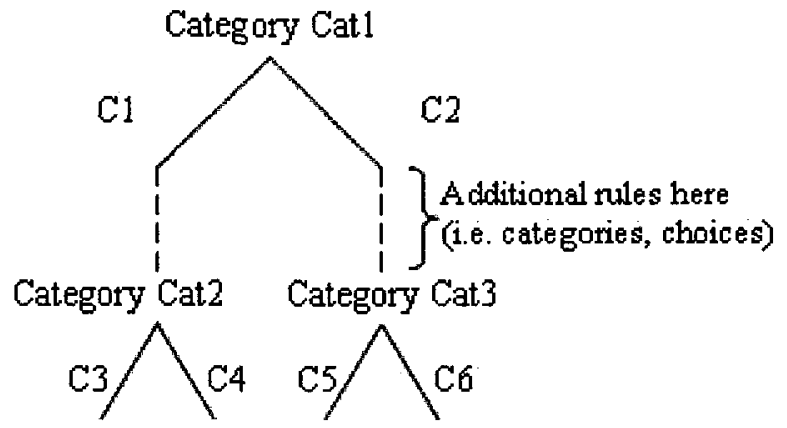

(b)

Figure 10 Adding Test Cases from the Tree

One advantage of this heuristic is that by using the information provided by the tree, when the tester intends to cover new choices, she does not have only the expensive option to exercise all the feasible combinations of choices, but can focus on combinations that are likely to affect the output.

\footnotetext{
${ }^{6}$ There is one exception though: if $\mathrm{C} 1$ is an error condition (e.g., an out of range input value), then $\mathrm{C} 1$ is not combined with $\mathrm{C} 5$ and $\mathrm{C} 6$. This is consistent with the CP strategy.
} 


\subsubsection{Removing Test Cases}

As discussed earlier, it is in general important to minimize functional test suites as ad hoc test suites often turn out to contain such redundancy. In our context, when a rule covers many test cases in a decision tree then the question arises whether they are all necessary. This means that a number of test cases exercise the same choice combinations for a subset of categories and then, as a result, fall in the same output equivalence class. The tester may then consider whether all these test cases in the same rule are necessary as they have similar properties, lead to similar outputs, and probably exercise the software in a similar fashion. Though this remains a subjective decision that only the tester can make; the decision tree points out potential redundancy. There may be, however, two reasons for redundancy: too many test cases for a rule (Section 4.3.6), ill-defined choices (Section 4.3.2).

The heuristic we follow when removing test cases is the following: we randomly remove test cases per leaf (rule) unless the percentage of code coverage drops. Obviously, different code coverage criteria can be used, and the impact of the possible criteria will have to be investigated. 


\section{CHAPTER 5 CASE STUDY ONE: PACKHEXCHAR}

In this section, we first describe the system used for the case study and the application of $\mathrm{CP}$ on this program (Sections 5.1 and 5.2). We then present the design of the case study (Section 5.3) which includes the comparison of CP specifications obtained with MELBA and CP specifications created by experts (Section 5.4). Sections 5.5 and 5.6 describe the results of applying C4.5 decision trees to drive the improvement of the test specifications and test suites. Lastly, we assess the fault detection effectiveness of the improved test suites in section 5.7.

\subsection{The PackHexChar Program}

PackHexChar is a Java adaptation of the sreadhex procedure, used in the GhostScript program and described in [28], to manipulate hexadecimal characters. The program contains 54 NBNC Java code lines. PackHexChar takes a string of characters representing hexadecimal digits (parameter S) and compacts the representation of the string in binary format (output), specifically as an array of Bytes: e.g., string " $34 \mathrm{AB}$ ", corresponding to binary values $0011,0100,1010$, and 1011 , is compacted into an array of two Byte values 00110100 and 10101011 (the binary representation of hexadecimal characters ' 3 ' and ' 4 ' are combined into the first byte value 00110100 ). In the input string, characters other than hexadecimal ones are ignored. In addition to the array of Bytes, the program returns an integer value. If the input string contains an even number of hexadecimal characters, pairs of hexadecimal characters are compacted, the program returns the array of Bytes and the returned integer value 
equals to -1 . If the input string contains an odd number of hexadecimal characters, an even number of characters is compacted, and the program returns the remaining hexadecimal character. The user can decide to look at only a sub-string of the input string S, using the input parameter RLEN: the RLEN first characters of S are then analyzed. If RLEN is not a legal value (negative or greater than S's length), the program returns value -2 . The user can ask the program to append an hexadecimal character at the beginning of S. This is useful when a string is split and analyzed in pieces with repeated calls to PackHexChar: a call can return a trailing hexadecimal character, which has to be appended at the beginning of the string during the next call. This is done with input parameter ODD_DIGIT. An ODD_DIGIT value of -1 indicates that no character is to be appended. If ODD_DIGIT has an illegal value (strictly below -1 or not an hexadecimal value), the program returns -3 .

\subsection{Application of Category-Partitioning}

We used Category-Partition on the PackHexChar program thereby identifying eleven categories and 35 choices (Appendix A.2). (Note that we did not identify constraints and selectors, as required when applying category-partition [6], since we did not use the technique to devise test cases, but to characterize test cases.). In Appendix A.2, parameter RLEN has two categories and four choices, parameter ODD_DIGIT has two categories and six choices, and parameter $\mathrm{S}$ has seven categories and 25 choices.

The output parameter is divided into the following equivalence classes: $-1,-2,-3$, $\mathrm{S}[\mathrm{rlen-1]}$, and Odd_digit. Output equivalence classes $-1,-2$, and -3 are selfexplanatory as they are errors. Output equivalence class $\mathrm{S}[\mathrm{rlen-1]}$ corresponds to 
the case where an odd number of hexadecimal characters are packed: the function returns the last character to consider in the string, i.e., the character at position rlen1 in string S. Output class Odd_digit is for the case where no hexadecimal character is packed but input parameter ODD_DIGIT contains one, in which case this character is returned.

\subsection{Design of the Case Study}

Our case study took place in the context of a specialized $4^{\text {th }}$ year course on software testing. The students were properly trained regarding white and black-box testing techniques, including Category-Partition. Recall from the introduction that the MELBA method we propose can be applied in two broad contexts: The reuse, validation, and integration of open source software, and software evolution. We can however further distinguish two situations that require we perform two slightly different types of case studies.

The first situation is when the $\mathrm{CP}$ specification is used to generate the test suite and the test suite must evolve to account for changes in the system under test (Evolution context). To emulate this situation, we asked a group of students to devise a $\mathrm{CP}$ specification and derive a test suite from it. We then generated the abstract test suites using each student's CP specification and test suite and followed the MELBA process. The results are reported in Section 5.5.

In the second situation we assume we have the test suite but no CP specification (e.g., a typical situation for Open Source Software-OSS). Tester must then reverseengineer the CP specification based on their understanding of the software functional 
behavior and then iteratively refine it. To emulate this situation (referred to as OSS context) we developed our own $\mathrm{CP}$ (referred to as expert $C P$ ). We applied this $\mathrm{CP}$ specification to students' test suites and followed the MELBA process. The results are reported in Section 5.6.

Please note that in our case studies we are applying the Melba method. We have not asked the students to do that because of time constraints. By doing so we ensure the Melba process is applied correctly and evaluate its advantages and drawbacks when applied correctly. Future work will evaluate how easy the Melba process can be applied by students.

In both contexts, we selected a subset of the student's work to apply our method. In each context we selected three students' work, making sure to have one poor application of CP (and poor TS), one average application of CP (and average TS), and one good application of CP (and average TS). The criteria used to identify a poor, average and good CP is based on the quality of the categories and choices compared to those of the Expert CP. Test suites are categorized by the number of test cases they contain. In the OSS context we also made sure to select three students other than the one selected for the Evolution context. Students are referred to as students A, B, and $\mathrm{C}$, and $\mathrm{X}, \mathrm{Y}$, and $\mathrm{Z}$, in those two contexts, respectively.

In both contexts, we then tried to identify occurrences of problems using the heuristics described in Figure 7. For both contexts, we actually went through the iterative improvement process illustrated in Figure 4: Both test suites and CP specifications were iteratively improved using the patterns we specified in Section 
4.3 for analyzing decision trees and their potential relationships to problems in the test suite or CP specification. Due to time constraints, we did not ask the students to go through the iterative process themselves. Instead we, ourselves, went through the process. At the end of the application of the MELBA process, we compare the (improved) $\mathrm{CP}$ specification against the expert $\mathrm{CP}$ : section 5.4 specifies how we compare CP specifications.

Since we also require code coverage information when removing test cases, Emma [29], an open source toolkit for measuring and reporting java code coverage, is used for our purpose. Emma supports class, method, line (statement) and basic block coverage, aggregated at package, source file, class, and method level. In this case study we use line (statement) coverage to analyze the results.

Last, since the purpose of our approach is to improve a test suite, e.g., to gain more confidence into an open source component, we are interested into whether the test suites we obtain at the end of the iterative process are able to find more faults than the initial test suite. To do so, we generated mutant versions of the PackHexChar program, i.e., faulty versions of the program, and evaluated how many of those mutants (faults) are actually caught by the test suites. This is discussed in section 5.7.

\subsection{Comparing Iteration results with Experts'}

One important analysis in our case studies is to compare the $\mathrm{CP}$ specifications obtained by the expert with the ones obtained after refining the students' $\mathrm{CP}$ specifications. We first specify how we intend to compare CP specifications (section 5.4.1), and then illustrate the comparison on an example (section 5.4.2). 


\subsubsection{Comparing CPs}

Recall that a category is a major property or characteristic of a parameter or environment condition of the program that describes how the program behaves with respect to some characteristic of the parameter or environment condition. Each category is decomposed into distinct choices that include all the different kinds of values that are possible for the category. Each choice within a category is therefore "a set of similar values that can be assumed by the type of information in the category." [6]

When $\mathrm{C} 4.5$ builds a decision tree, not all the categories and choices initially defined by the tester in the Category-Partition specification are necessarily used to classify the instances. Some categories and choices will not appear in the tree if they do not determine the program output equivalence classes. This may simply be due to the granularity of the output equivalence classes and may not therefore denote a problem in the Category-Partition specification ${ }^{7}$. When comparing Category-Partition specifications, since our goal is to verify the behavior of the program under test to the level of granularity determined by these output equivalence classes, we only consider

\footnotetext{
${ }^{7}$ For instance, in the expert CP (Appendix A.2) Category 10 is about the position of the first nonhexadecimal character in the first $r$ len characters of $s$ : the (expert) tester considered that the program may not be able to adequately omit non-hexadecimal characters if they are at the beginning of string $\mathrm{S}$. This category does not appear in any tree since it does not determine the program output equivalence classes we have selected. Similarly, Category 11 is about the number of consecutive non-hexadecimal characters in the first $r$ len characters of $s$ : the tester considered that omotting one non-hexadecimal character and omitting several consecutive ones would be two situations worth testing. This category does not appear in any tree.
} 
categories and choices that appear in decision trees, that is the categories and choices which seem to have an influence on the identified output equivalence classes.

To compare two Category-Partition specifications, let us first introduce some formalism. We refer to one application of Category-Partition as CP. CP then refers to the categories and choices that are defined by the tester. Let Dт be a decision tree generated by $\mathrm{CP}$ and test suite $\mathrm{TS} . \mathrm{CP}_{\mathrm{Dr}}$ then denotes the subset of the categories and choices of CP that appear in DT. Label cat refers to a category, ch refers to a choice and we consider $\mathrm{CP}$ as a set of categories and choices: e.g., if category cat belongs to Category-Partition specification CP, then we write cat $\in \mathrm{CP}$. Similarly, we consider that a category is a set of choices: e.g., if choice ch is a choice of category cat, then we write ch $\in$ cat. Given that a choice is "a set of similar values that can be assumed by the type of information in the category" [6] we use set theory to compare choices: e.g., if choice c represents a set of values (assumed by the type of information of a category) that is included in the set of values of choice $c^{\prime}$, then we write $c \subseteq c^{\prime}$. When the domain of values of a choice ch in category cat is covered by the domains of values of one or more choices in category cat', we say that choice ch in cat is covered by one or more choices $c h_{\mathrm{i}}{ }^{\prime}$ in cat' (denoted $\left.c h \subseteq \bigcup_{i} c h_{i}^{\prime}\right)$. We denote the determination of output equivalence classes $\mathrm{O}$ based on choice ch as function $\mathrm{DT}_{0}(\mathrm{ch}): \mathrm{DT}_{0}(\mathrm{ch})$ is the set of output equivalence classes in $\mathrm{O}$ of all the rules in which choice ch appears. For a given decision tree $\mathrm{DT}$, keeping choices in other categories constant, $\mathrm{DT}_{\mathrm{o}}(\mathrm{choice} 1)=\mathrm{DT}_{\mathrm{o}}($ choice 2$)$ implies that whether one choice 
or the other is selected does not matter as far as the defined equivalence classes are concerned.

Definition: Category-Partition specification equivalence Given two category-partition specifications $\mathrm{CP}$ and $\mathrm{CP}^{\prime}$, we say that $\mathrm{CP}$ and $\mathrm{CP}$ ' are equivalent with respect to a given set of output equivalence classes $O$ (denoted $C P \cong_{O} C P^{\prime}$ ) if and only if, given the two decision trees $\mathrm{DT}_{1}$ and $\mathrm{DT}_{2}$ generated from CP and CP' respectively (i.e., from different test suites), for each category cat in $C P_{D T 1}$ there is a category cat' in $C P_{D T 2}^{\prime}$ such that each choice ch in cat is covered by one or more choices $\mathrm{ch}_{\mathrm{i}}{ }^{\prime}$ in cat' such that differences in the definitions of choices do not affect the determination of the output equivalence classes in $\mathrm{O}$.

$$
C P \cong_{O} C P^{\prime} \Leftrightarrow\left\{\begin{array}{l}
\forall c a t \in C P_{D T 1} \exists c a t^{\prime} \in C P_{D T 2}^{\prime} \mid \\
\left(\forall c h \in c a t \exists c h_{1}^{\prime}, \ldots, c h_{n}^{\prime} \in c a t^{\prime} \mid c h \subseteq \bigcup_{i} c h_{i}^{\prime}\right) \\
o r \\
\left(\forall c h^{\prime} \in c a t^{\prime} \exists c h_{1}, \ldots, c h_{m} \in c a t \mid c h^{\prime} \subseteq \bigcup_{i} c h_{i} \text { and } D T_{O}\left(c h_{i}^{\prime}\right)=D T_{O}(c h)\right)
\end{array}\right.
$$

In the formal definition above, $\mathrm{CP}_{\mathrm{Dr} 1}$ are the categories and choices selected by $\mathrm{C} 4.5$ to construct $\mathrm{DT} 1$ and $\mathrm{CP}_{\mathrm{DT} 2}$ are the categories and choices selected by $\mathrm{C} 4.5$ to construct DT2. Choices of categories in $\mathrm{CP}_{\mathrm{Dr}}$ may be covered by one or more choices of categories in $\mathrm{CP}_{\mathrm{DT} 2}$ (case 1), or choices of categories in $\mathrm{CP}_{\mathrm{DT2}}$ may be covered by one or more choices of categories in $\mathrm{CP}_{\mathrm{Dr} 1}$ with the same output equivalence class (case 2).

Note that the CP specification equivalence definition assumes that the two decision trees being compared have comparable sets of choices: i.e., a choice of a tree is 
always included in a choice of the other tree, or the other way round. This means that we assume the improvement process discussed in Figure 4 leads to such comparable situation. These assumptions will be confirmed by experiments.

\subsubsection{CP specification comparison: an example}

As an example, we compare the categories and choices of the expert and one student's Category-Partition specifications (student $\mathrm{C}$ ). Figure 11 shows $\mathrm{CP}_{\mathrm{Dr}}$ of both the expert and student C's specifications. First note that Figure 11 does not show all the categories and choices of the expert and student CP specifications. This is to be expected since, as discussed previously, we only consider categories and choices that appear in decision trees.

Choice $\mathrm{C} 1(\mathrm{rlen}=0)$ in the expert $\mathrm{CP}_{\mathrm{Dr}}$ represents a set of values assumed by the type of information in Category 1. It is the same set as choice C1 (\{0\}) in student C's $\mathrm{CP}_{\mathrm{DT}}$ (case 1). Choice $\mathrm{C} 2(\mathrm{rlen}=[1 \ldots \mathrm{sLength}])$ in the expert $\mathrm{CP}_{\mathrm{DT}}$ represents a set of values assumed by the type of information in category 1 . It is the same set as choice $\mathrm{C} 2([1$ - s. length $])$ in student $\mathrm{C}^{\prime} \mathrm{S} \mathrm{CP}_{\mathrm{Dr}}$ (case 1). Choices $\mathrm{C} 3$ and $\mathrm{C} 4$ of category 2 in the expert $\mathrm{CP}_{\mathrm{Dr}}$ represents a set of values ( $\mathrm{rlen}<0$ or $\mathrm{rlen}>$ sLength) that is included in the set of values represented by choice $\mathrm{c} 3$ of category 1 (anything else than $\{0\}$ and $[1-$ s.length $]$ ) in student $C^{\prime} \mathrm{S}_{\mathrm{DP}}$ (case 1). Choice $\mathrm{C} 5$ in the expert $\mathrm{CP}_{\mathrm{DT}}$ is the same set as choice $\mathrm{C} 4$ in student $\mathrm{C}^{\prime} \mathrm{S} \mathrm{CP}_{\mathrm{Dr}}$ (case 1). The set of values represented by choices $\mathrm{C} 6, \mathrm{C} 7$, and $\mathrm{C} 8$ of category 3 in the expert $\mathrm{CP}_{\mathrm{DT}}$ is included in the set of values represented by choice $\mathrm{C} 5$ of category 2 in student $\mathrm{C}^{\prime} \mathrm{S}_{\mathrm{DT}}$ (case 1). The set of values represented by choices $\mathrm{C} 9$ and $\mathrm{C} 10$ of category 4 in the expert $\mathrm{CP}_{\mathrm{Dr}}$ is 
included in the set of values represented by choice $\mathrm{C} 6$ of category 2 in student $\mathrm{C}^{\prime} \mathrm{SP}_{\mathrm{DT}}$ (case 1). Choice $\mathrm{C} 28, \mathrm{C} 29$, and $\mathrm{C} 30$ in the expert $\mathrm{CP}_{\mathrm{Dr}}$ represents a set of values assumed by the type of information in category 9 which is the same set as the one of choices $\mathrm{C} 12, \mathrm{C} 13$, and $\mathrm{C} 14$ in student $\mathrm{C}$ 's $\mathrm{CP}_{\mathrm{DT}}$ (case 1).

\begin{tabular}{|c|c|}
\hline Expert CP & student $C^{\prime} \& C P$ \\
\hline 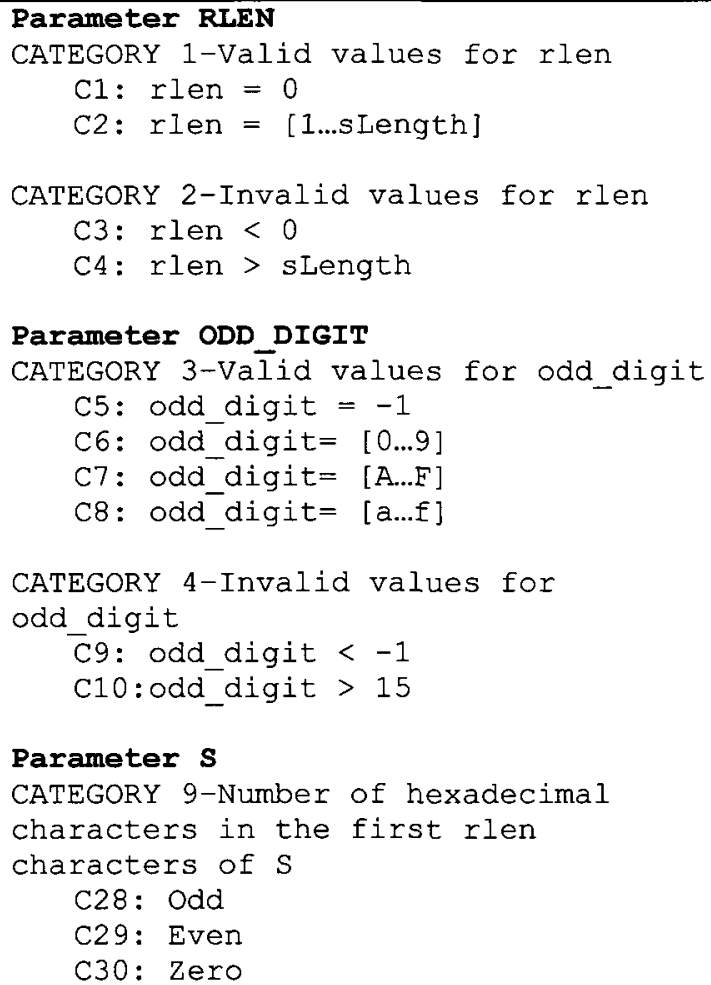 & $\begin{array}{l}\text { Parameter: RLEN } \\
\text { Category 1: number of characters to } \\
\text { read } \\
\text { C1: }\{0\} \\
\text { C2: [1 - s.length] } \\
\text { C3: anything else } \\
\text { Parameter: oDD_DIGIT } \\
\text { Category 2: Extra digit to parse } \\
\text { C4: \{-1\} } \\
\text { C5: [0 - 15] } \\
\text { C6: anything else } \\
\text { Parameter: s } \\
\text { Category 5: Number of valid hex } \\
\text { characters from } 0 \text { to rlen } \\
\text { C12: Odd } \\
\text { C13: Even } \\
\text { C14: Zero }\end{array}$ \\
\hline
\end{tabular}

Figure 11 CP for the Expert CP and Student C's CP

Conversely, we can compare the categories and choices of student C's CategoryPartition specification with the Expert Category-Partition specification. Choice C1 $(\{0\})$ in the student $\mathrm{CP}_{\mathrm{DT}}$ represents a set of values assumed by the type of information in Category 1. It is the same set as choice $\mathrm{C} 1(\mathrm{rlen}=0)$ in the expert $\mathrm{CP}_{\mathrm{DT}}$ (case 1). Choice $\mathrm{C} 2([1-\mathrm{s}$. length $])$ in the stduent $\mathrm{CP}_{\mathrm{Dr}}$ represents a set of values assumed by the type of information in category 1 . It is the same set as choice $\mathrm{C} 2$ (rlen = [1...sLength] $)$ in the expert $\mathrm{CP}_{\mathrm{Dr}}$ (case 1). Choice $\mathrm{C} 3$ of category 1 in the student $\mathrm{CP}_{\mathrm{Dr}}$ 
(anything else than $\{0\}$ and $[1-$ s.length $]$ ) includes the set of values represented by choice $\mathrm{C} 3$ and $c 4$ ( $r$ len $<0$ or rlen $>$ sLength) of category 2 in the expert $\mathrm{CP}_{\mathrm{DT}}$. And the set of output equivalence classes of $\mathrm{C} 3$ and $\mathrm{C} 4$ in the expert $\mathrm{CP}$ equals the set of output equivalence classes of $\mathrm{C} 3$ in the student $\mathrm{CP}$ (case 2). Choice C4 $(\{-1\})$ in the student $\mathrm{CP}_{\mathrm{DT}}$ represents a set of values assumed by the type of information in Category 1. It is the same set as choice C5 (odd_digit $=-1$ ) in the expert $\mathrm{CP}_{\mathrm{DT}}$ (case 1). Choice $\mathrm{C} 5([0-15])$ of category 2 in student $\mathrm{C}^{\prime} \mathrm{S} \mathrm{CP}_{\mathrm{DT}}$ includes in the set of values represented by choices $c 6, c 7$, and $c 8$ of category 3 in the expert $\mathrm{CP}_{\mathrm{Dg}}$, and the output equivalence classses are the same (case 2). Choice $\mathrm{C} 6$ of category 2 in student $C^{\prime} S \mathrm{CP}_{\mathrm{DT}}$ includes the set of values represented by choices $\mathrm{C} 9$ and $\mathrm{C} 10$ of category 4 in the expert $\mathrm{CP}_{\mathrm{DT}}$ (case 1). Choices $\mathrm{C} 12, \mathrm{C} 13$, and $\mathrm{C} 14$ in student $\mathrm{C}$ 's $\mathrm{CP}_{\mathrm{DT}}$ represent sets of values assumed by the type of information in category 9 which are the same sets as the ones of choices $\mathrm{C} 28, \mathrm{C} 29$, and $\mathrm{C} 30$ in the expert $\mathrm{CP}_{\mathrm{DT}}$ (case 1).

Therefore expert CP is equivalent to student C's CP. Appendix B gives a mapping between categories and choices of the Expert CP and other students' CPs.

\subsection{Results with Student's CP-Evolution Context}

\subsubsection{Student A's CP and TS}

Student A's test suite contains 20 test cases. We created 20 abstract test cases using A's Category-Partition specifications (Appendix A.3). Executing C4.5 on these abstract test cases, we obtain the decision tree (and confusion matrix) of Figure 12 made of 12 rules (following the WEKA format for displaying trees). This tree shows a number of issues (bold face). First, there is one misclassification (a test case with 
output equivalence class $S[r l e n-1]$ is misclassified as having output equivalence class -1.0 ): Case 1 in Section 4.2. An analysis of the program specification and test suite shows that a category is missing (Section 4.3.1): The student failed to realize that only the RLEN first characters are compacted (Section 5.1). The tree also shows missing combinations of choices (Case 3), e.g., allValid (in category SCHARTYPE) is combined with odd_digit=-1 (Rule1) but not with odd_digit=[0...15] (rules 7, 8, 9). Some missing combinations are even explicit as they correspond to rules with no instance (three examples identified by $(0.0)$ in bold face: rules 3,5 , and 9 ), these are, however, unfeasible choice combinations (Section 4.3.5). For instance, rule 3 is unfeasible because if "SLENGTH = EvenLength" i.e. the length of the string is even, then the type of characters in the string (i.e. SCHARTYPE) either has to be allvalid or Mixed (Mixed represents a mix of valid and invalid characters or all invalid characters), and never "N/A". Hence rule 3 is an unfeasible combination of choices. Rule 5 and 9 are unfeasible because choices " $r$ len= $[1 \ldots$ Slength $]$ " and "SLENGTH $=$ Empty" contradict each other, hence leading to unfeasible combinations. 


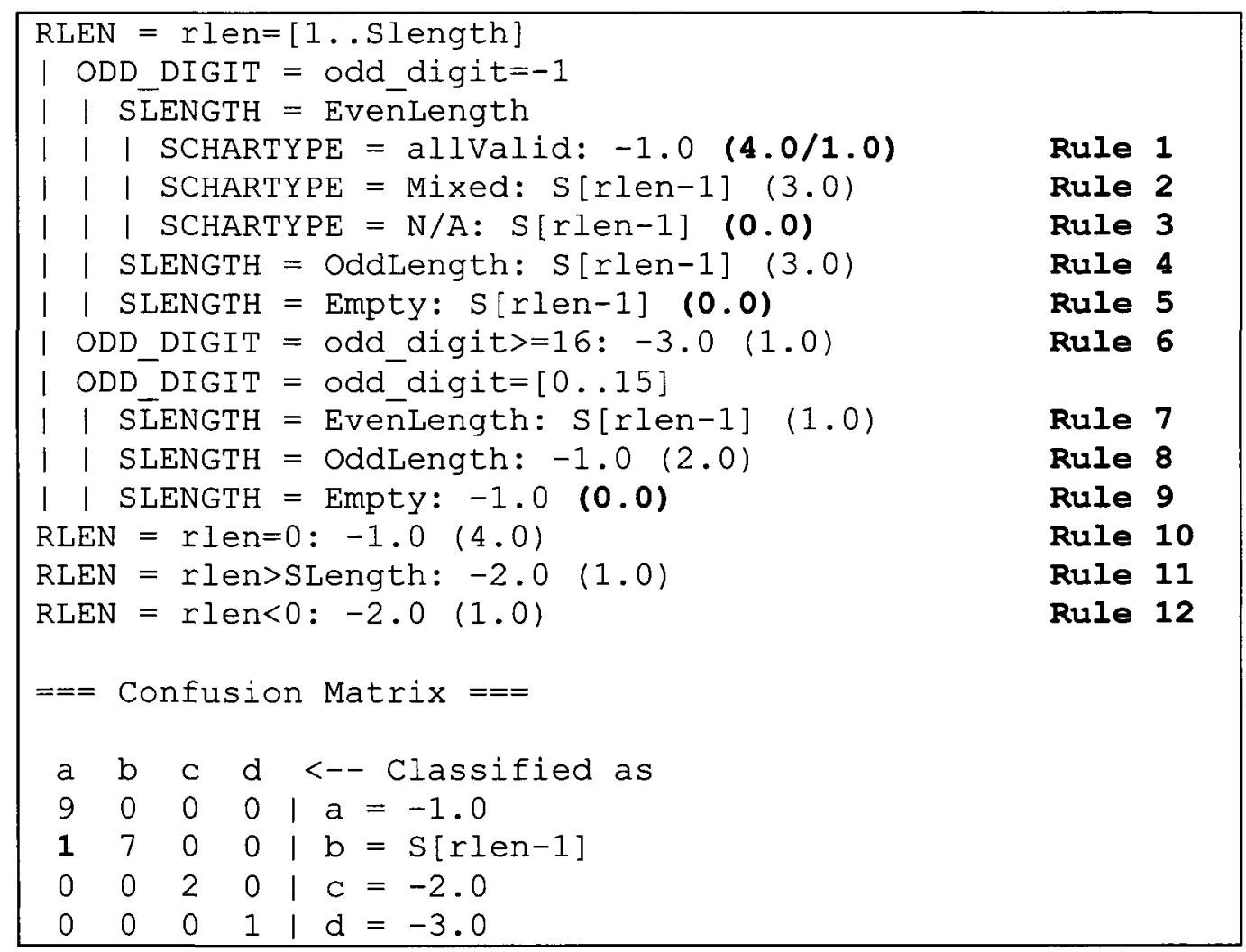

Figure 12 Student A's TS + A's CP - Iteration 1

We first add the following missing category (Activity 5, Figure 4) (Recall section 5.3 that it is the expert who applies the Melba method):

Category: Number of hexadecimal characters in the first rlen characters of input string $s$ Choice 1: Odd Choice 2: Even Choice 3: Zero

Once the abstract test cases are (automatically) re-created from the updated $\mathrm{CP}$, the execution of $\mathrm{C} 4.5$ produces the decision tree of Figure 13 made of 10 rules. As expected, the tree no longer shows misclassification. However, it shows that some choice combinations are missing (Case 3), two of them being explicitly identified in rules with no instance (rules 3 and 7). Rule 7 is an unfeasible combination of choices (Section 4.3.5) because choices "rlen=[1...Slength]" and "SLENGTH = Empty" 
contradict each other, whereas rule 3 is a feasible (missing) combination indicating of a missing test case (Case 2.2).

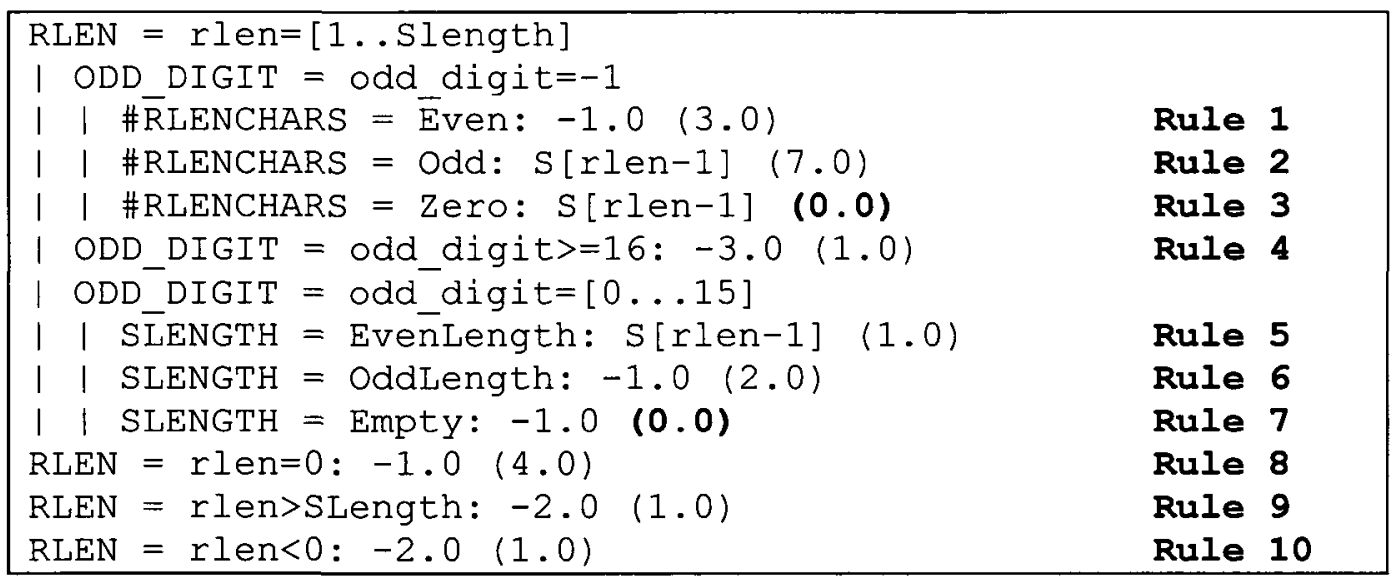

Figure 13 Student A's TS + A's CP - Iteration 2

Following the heuristic described in Section 4.4, one can identify the following 15 missing combinations of choices in the decision tree, nine of which are already exercised by the 20 original test cases. The six missing combinations of choices for which there is no test case in the test suite are identified with a '*'.

\begin{tabular}{|c|c|}
\hline 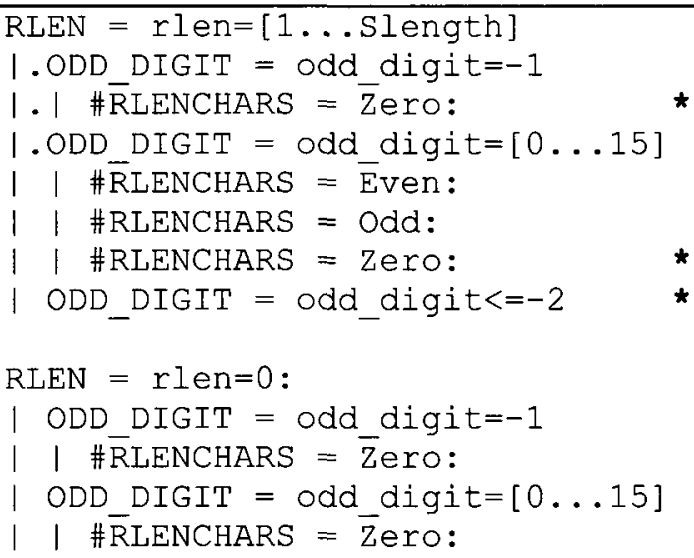 & 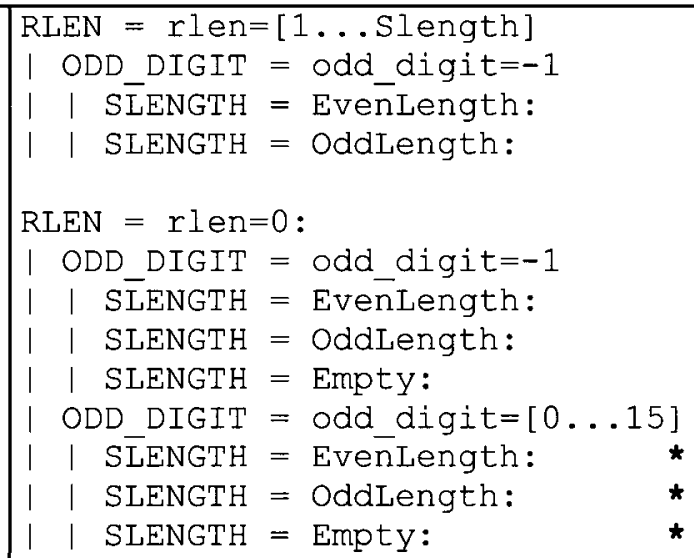 \\
\hline
\end{tabular}

Test cases are added for the above six missing combinations of choices. Abstract test cases are created and C4.5 is re-run, producing the decision tree of Figure 14 made of 14 rules. There is no misclassification, but a number of rules have zero instances (rules 2, 4, and 8 ), or the number of instances is large which could therefore indicate 
redundant test cases (Case 4). Rules 2 and 4 with no instance are unfeasible choice combinations (Case 3.1), because it is not possible for the number of the first rlen characters in string $S$ to be even "RLENCHARS = Even" and rlen to be zero or less than zero. Rule 8 with zero instances is feasible, suggesting a missing test case (Case 2.2). Since this choice combination involves an error condition (illegal input value rlen>SLength) which is already used in a test case (Rule 3 in the tree), we do not consider this a missing combination of choices (Case 2.2 and footnote 6 on page 46), but since it is still a feasible combination it appears in the tree. The tree also suggests that the following five combinations of choices are missing:

\begin{tabular}{|c|c|}
\hline $\begin{array}{l}\text { ODD DIGIT }=\text { odd digit }=-1 \\
\text { |\#RLENCHARS }=\overline{\text { Odd }} \\
\mid \text { | RLEN }=\text { rlen= }[1 \ldots \text { slength }]: 1\end{array}$ & $\begin{array}{l}\text { ODD DIGIT }=\text { odd digit }=[0 \ldots 15] \\
\text { | \#RLENCHARS }=\text { Even } \\
\mid \text { | RLEN }=\text { rlen= }[1 \ldots \text { Slength }]: \\
\text { | \#RLENCHARS }=\text { odd } \\
\mid \text { RLEN }=r l e n=[1 \ldots \text { Slength }]: \\
\text { \#RLENCHARS }=\text { zero: } \\
\text { | RLEN }=r \text { ren= }[1 \ldots \text { Slength }]: \\
\mid \text { RLEN }=\text { rlen=0: }\end{array}$ \\
\hline
\end{tabular}

These combinations are however already exercised in the 26 test cases ${ }^{8}$, and Figure 14 is therefore the final decision tree.

\footnotetext{
${ }^{8}$ For instance, all the seven test cases in rule 5 satisfy $r l e n=[1 \ldots$ slength]. The other choices of RLEN are not combined with this choice of RLENCHARS (Odd) as they either involve an error choice that is already exercised once in the test suite (i.e., $r l e n<0$ and $r l e n>$ SLength) or it does not make sense to combine them (combining \#RLENCHARS=Odd and $r l e n=0$ does not make sense: we compact 0 characters in the string and therefore the number of hexadecimal characters in the string does not matter).
} 


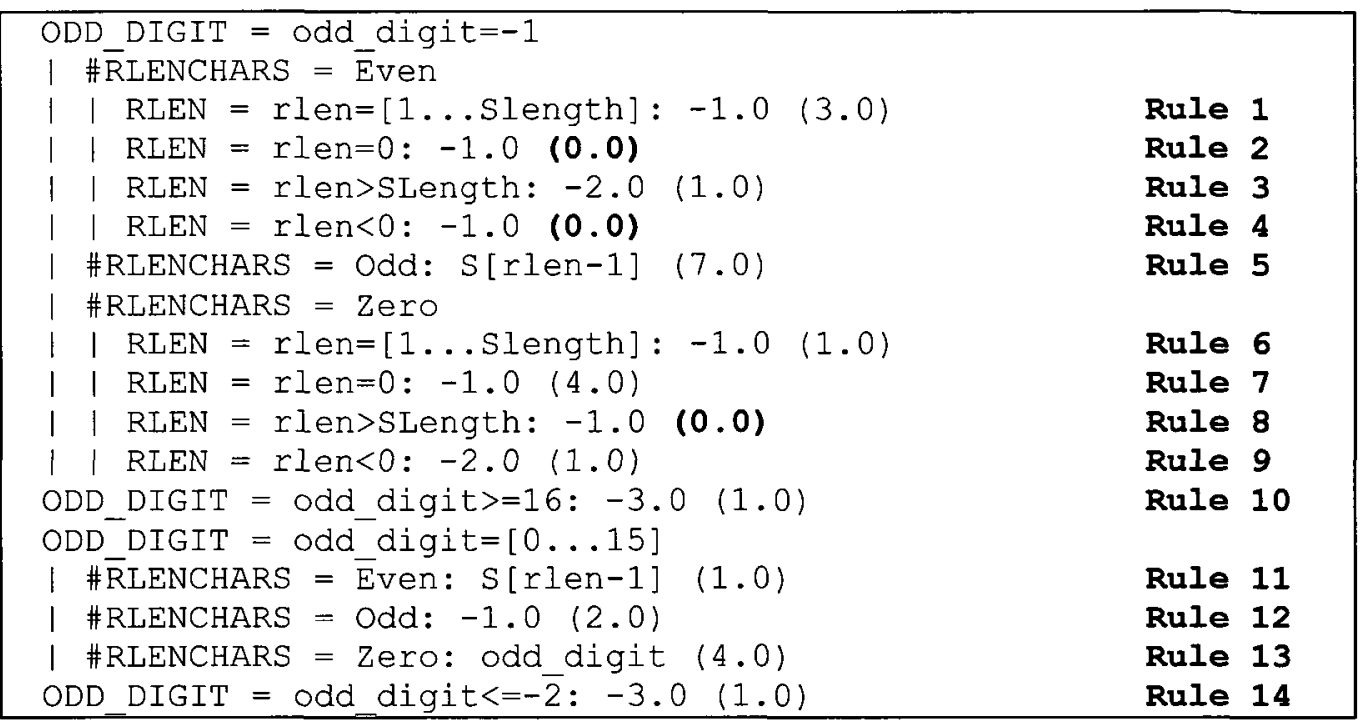

\section{Figure 14 Student A's TS + A's CP - Iteration 3}

The final student $\mathrm{CP}$, which contains eight categories and 16 choices, of which categories 1, 2, 3, 4, and 7 (i.e. five categories and eleven choices) are used in the tree, is equivalent to the expert $\mathrm{CP}$ (section 5.4.1).

Depending on time and resource constraints, as a final step to improve the test suite, one may want to reduce the number of test cases (instances) because some rules cover more than one instance (Section 4.3.6).

We removed some of the test cases in those rules (randomly selected), keeping one test case for each one of those rules while still maintaining the same code coverage. The final decision tree is shown in Figure 15 with 14 rules. 


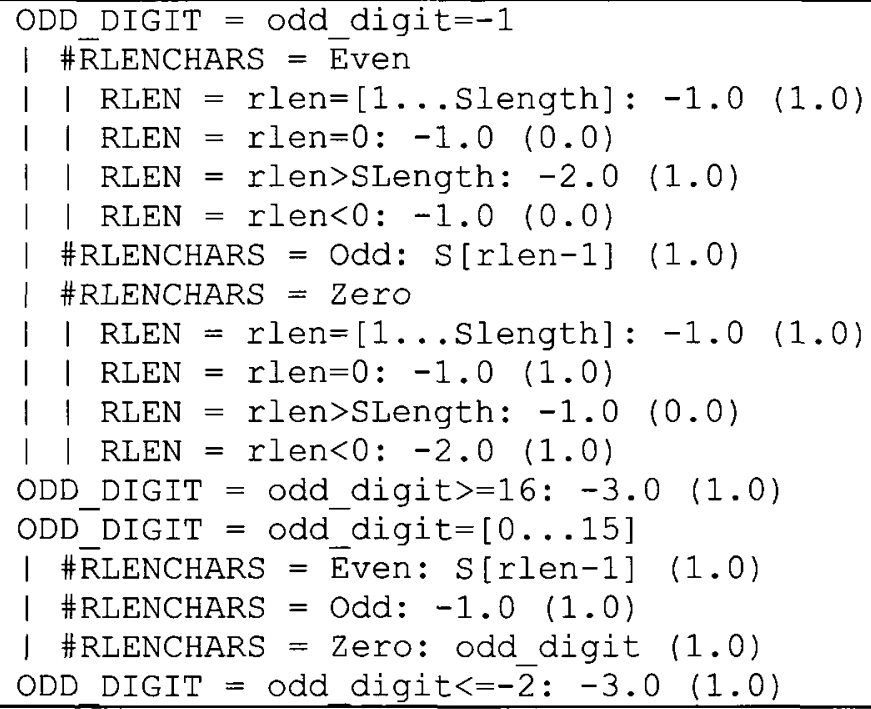

Figure 15 Student A's TS + A's CP - Final Tree

\subsubsection{Student B's CP and TS}

Student B's test suite contains 31 test cases. We created 31 abstract test cases using B's Category-Partition specifications (Appendix A.4). Executing C4.5 on these abstract test cases, we obtain the decision tree (and confusion matrix) of Figure 16 with 14 rules. The decision tree shows eight misclassified test cases (Case 1, rules 2, 5 and 11); six instances of $S[r l e n-1]$ are misclassified as -1 and two instances of -1 are classified as $S[r l e n-1]$. This again is due to the student failing to recognize that the program compacts the first RLEN hexadecimal characters in the input string, resulting in a missing category in student B's $\mathrm{CP}$ (Case 1.1). Following the heuristic described in Section 4.4, the following combinations of choices are also missing in the decision tree (Case 3): 


\begin{tabular}{|c|c|}
\hline $\begin{array}{l}\text { ODD_DIGIT }=\text { odd digit }=-1 \\
\text { SLENGTH }=\text { oddLength: } \\
\text { | SLENGTH }=\text { EvenLength: } \\
\text { SLENGTH }=\text { Empty: } \\
\text { ODD_DIGIT = odd_digit=-1 } \\
\text { RLEN = rlen=0: } \\
\text { RLEN = 0<rlen<sLength } \\
\text { | SLENGTH VS. RLEN = sLength }>\text { rlen } \\
\text { I I SCHARTYPE = N } / \text { A: } \\
\text { I I SCHARTYPE = allinvalid: } \\
\text { RLEN = rlen<0: }\end{array}$ & 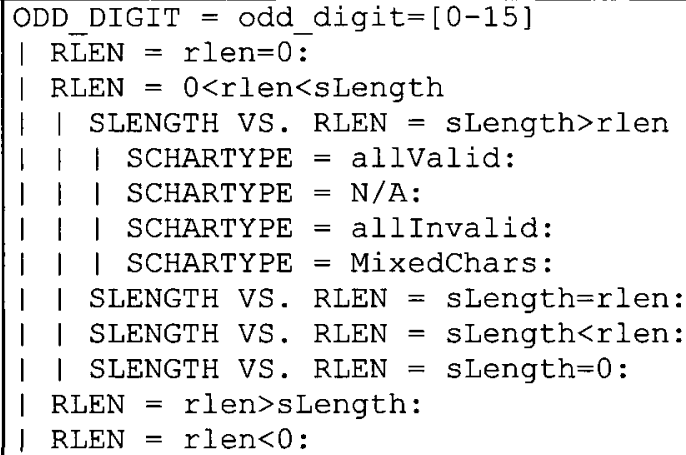 \\
\hline
\end{tabular}

Some of them are actually identified in the tree but they have a number of instances equal to zero (rules $3,4,7$, and 8). Rules 3 and 4 are feasible combinations and indicate missing test cases (Case 2.2). Rules 7 and 8 are unfeasible combinations of choices (Case 3.1), because choice sLength $<$ rlen of rule 7 and choice sLength $=0$ of rule 8 is in contradiction with the choice $0<r l e n<=s L e n g t h$. The decision tree also shows a missing choice $(r l e n<0)$ and is due to missing test cases (Case 2.2).

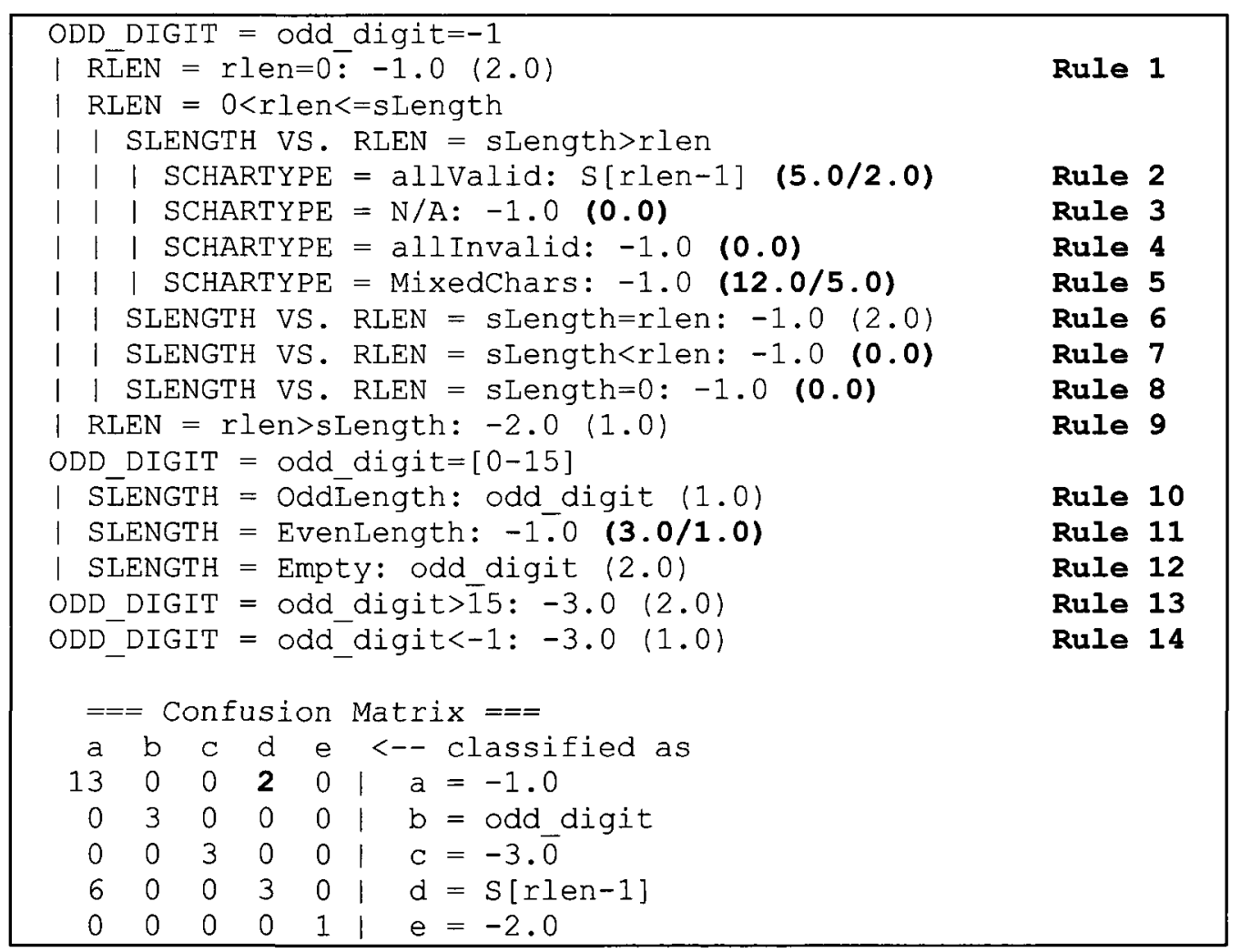

Figure 16 Student B's TS + B's CP - Iteration 1 
We first add the missing category to the student's $\mathrm{CP}$ (the same category as for student A). Once the abstract test cases are (automatically) re-created from the updated Category-Partition, the execution of $\mathrm{C} 4.5$ produces the decision tree of Figure 17 with 10 rules.

The tree in Figure 17 shows one rule with no instance (rule 3): this is an unfeasible combination of choices (Case 3.1), because the number of first rlen characters of string s can not be even (“\#RLENCHARS = EVEN") if rlen is zero ( $r l e n=0)$. The tree also suggests that the following eight combinations of choices are potentially missing:

\begin{tabular}{|c|c|}
\hline $\begin{array}{l}\text { ODD DIGIT }=\text { odd_digit }=-1 \\
\text { \#RLENCHARS }=\bar{z} \in \text { Ro: } \\
\text { | RLEN }=\text { rlen=0: } \\
\text { | RLEN }=0<r l e n<=\text { sLength: } \\
\text { \#RLENCHARS = Odd: } \\
\text { | RLEN }=0<\text { rlen }<=\text { sLength: }\end{array}$ & $\begin{array}{l}\text { ODD DIGIT = odd digit=[0-15] } \\
\text { \#RLENCHARS }=\text { Zero: } \\
\mid \text { RLEN }=\text { rlen=0: } \\
\text { | RLEN }=0<\text { rlen }<=\text { sLength: } \\
\mid \text { RLEN }=\text { rlen }<0: \\
\text { \#RLENCHARS }=\text { Odd: } \\
\mid \text { RLEN }=0<\text { rlen }<=\text { sLength: } \\
\text { \#RLENCHARS = Even: } \\
\mid \text { RLEN }=0<\text { rlen }<=\text { sLength: }\end{array}$ \\
\hline
\end{tabular}

Looking at the test suite shows that none of the above combinations have been already exercised.

\begin{tabular}{|c|c|c|}
\hline \multicolumn{3}{|l|}{ ODD DIGIT $=$ odd digit $=-1$} \\
\hline \#促ENCHARS $=\bar{z}$ ero: $-1.0 \quad(6.0)$ & Rule & \\
\hline \#RLENCHARS $=$ Odd: $S[r l e n-1](8.0)$ & Rule & 2 \\
\hline \#RLENCHARS = Even & & \\
\hline $\mid$ RLEN $=$ rlen=0: $-1.0 \quad(0.0)$ & Rule & 3 \\
\hline $\mid$ RLEN $=0<r l e n<$ sLength: -1.0 & Rule & 4 \\
\hline $1 \mid$ RLEN $=$ rlen>sLength: $-2.0(1.0)$ & Rule & 5 \\
\hline ODD DIGIT $=$ odd digit $=[0-15]$ & & \\
\hline 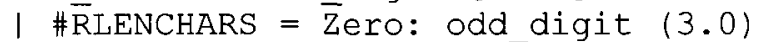 & Rule & 6 \\
\hline I \#RLENCHARS = Odd: $-1.0^{-}(2.0)$ & Rule & 7 \\
\hline I \#RLENCHARS = Even: $S[$ rlen-1] & Rule & 8 \\
\hline ODD DIGIT = odd digit>15: -3.0 (2.0) & Rule & 9 \\
\hline ODD DIGIT $=$ odd digit $<-1:-3.0 \quad(1.0)$ & Rule & 10 \\
\hline
\end{tabular}

Figure 17 Student B's TS + B's CP - Iteration 2

We therefore create eight test cases; (automatically) produce the corresponding abstract test cases and re-run C4.5, which returns the decision tree in Figure 18 with 
14 rules. The tree shows potentially missing choice combinations (rules 3,6 , and 9): rules 3 and 6 are unfeasible combinations of choices, because the number of first rlen characters of string $S$ can not be even ("\#RLENCHARS = EVEN") if rlen is zero $(r l e n=0)$ or if $r l e n$ is negative $(r l e n<0)$. Rule 9 is feasible but involves an error condition (rlen>sLength) which already appears in another rule. The tree also suggests missing combinations of choices. However, they are already exercised by the test suite and do not appear in the tree for the same reasons as for Student A. The tree shows rules with a number of instances larger than one (rules $1,2,4,7,8,11,12$, and 13), possibly suggesting redundant test cases.

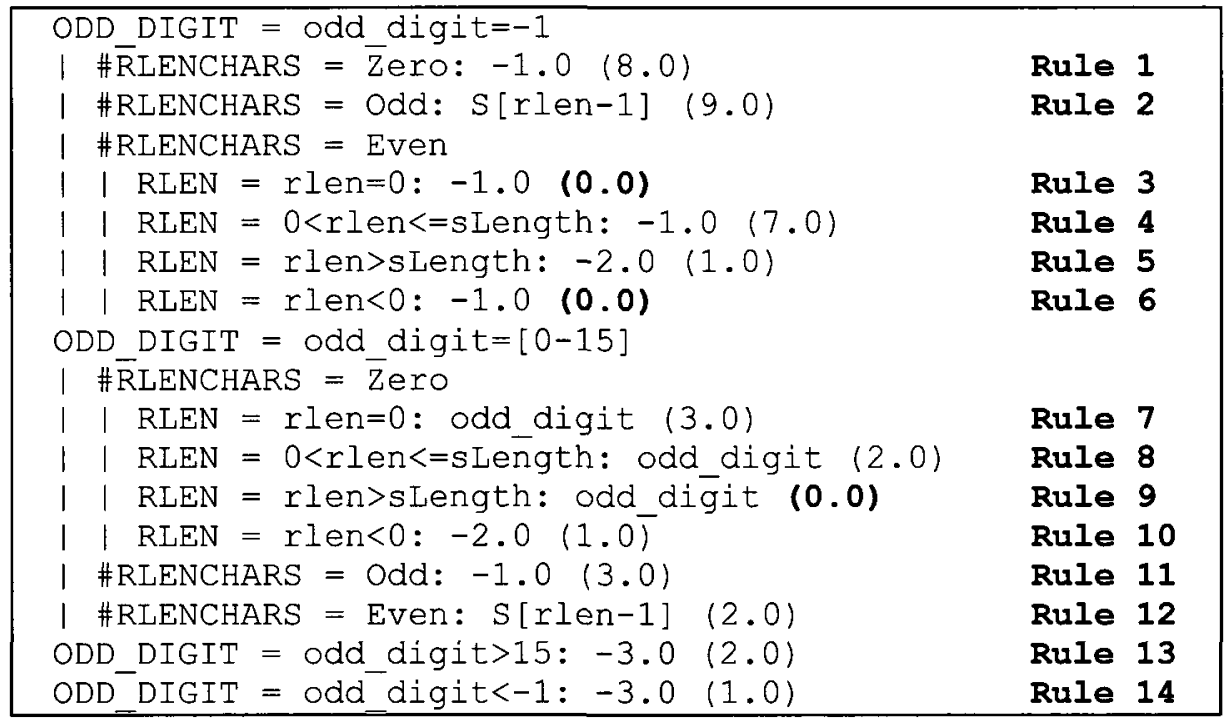

Figure 18 Student B's TS + B's CP - Iteration 3

We removed some of the test cases in those rules (randomly selected), keeping one test case for each rule except for rule 12 , because removing any further test case will result in reduced code coverage, thus maintaining the same code coverage. When rerunning $\mathrm{C} 4.5$, we obtain a tree with the same rules as in Figure 18 except that the number of instances for each rule (except rule 12) is now one. The final $\mathrm{CP}$, which 
contains nine categories and 23 choices of which categories $1,2,3,4$, and 8 (i.e. five categories and eleven choices) are used in the tree, is equivalent to the expert CP (Section 5.4.1).

\subsubsection{Student C's CP and TS}

Student C's test suite contains 19 test cases. Using C's CP specifications (Appendix A.5), 19 abstract test cases are created. After executing C4.5 on these abstract test cases, we obtain the decision tree (and confusion matrix) in Figure 19. The decision tree shows two misclassified test cases: rules 4 and 10 (Case 1). This again is due to the student failing to recognize that the program compacts the first RLEN hexadecimal characters in the input string, resulting in a missing category in student C'S CP (Case 1.1). There are no missing combinations of choices in the decision tree (Case 3, according to the heuristic described in Section 4.4). Rules 2 and 8 are unfeasible combinations of choices (Case 3.1), since it is not feasible to have "SCHARTYPE=N/A" and "RLEN $=[1 \ldots$ SLength $]$ " at the same time. 


\begin{tabular}{|c|c|c|}
\hline \multicolumn{3}{|l|}{ ODD $\mathrm{DIGIT}=\mathrm{ODD}$ DIGIT $=-1$} \\
\hline I $R \overline{\mathrm{L}} E \mathrm{~N}=\mathrm{RLEN}=0 \overline{\mathrm{s}}-1.0(2.0)$ & Rule & 1 \\
\hline I $\operatorname{RLEN}=\operatorname{RLEN}=[1 \ldots$ Slength $]$ & & \\
\hline I $\mid$ SCHARTYPE $=\mathrm{N} / \mathrm{A}:-1.0(0.0)$ & Rule & 2 \\
\hline I | SCHARTYPE = AllValid: S[rlen-1] (2.0) & Rule & 3 \\
\hline|| SCHARTYPE $=$ Mixed: $-1.0(2.0 / 1.0)$ & Rule & 4 \\
\hline | | SCHARTYPE = Allinvalid: $-1.0(2.0)$ & Rule & 5 \\
\hline | RLEN = RLEN=INVALID: $-2.0 \quad(1.0)$ & Rule & 6 \\
\hline \multicolumn{3}{|l|}{ ODD DIGIT $=$ ODD DIGIT $=[0 \ldots 15]$} \\
\hline 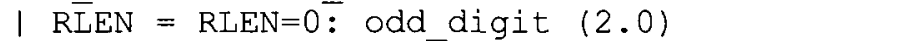 & Rule & 7 \\
\hline I RLEN = RLEN=[1...Sİength $]$ & & \\
\hline I | SCHARTYPE $=\mathrm{N} / \mathrm{A}:-1.0(0.0)$ & Rule & 8 \\
\hline | | SCHARTYPE = AllValid: -1.0 (2.0) & Rule & 9 \\
\hline | | SCHARTYPE = Mixed: $-1.0(2.0 / 1.0)$ & Rule & 10 \\
\hline | | SCHARTYPE = Allinvalid: odd digit (2.0) & Rule & 11 \\
\hline I RLEN = RLEN=INVALID: $-2.0 \quad(1.0)$ & Rule & 12 \\
\hline ODD_DIGIT $=$ ODD_DIGIT=INVALID: $-3.0(1.0)$ & Rule & 13 \\
\hline \multicolumn{3}{|l|}{$===$ Confusion Matrix $===$} \\
\hline$a b c d e<-c$ classified as & & \\
\hline $\begin{array}{lllllll}8 & 0 & 0 & 0 & 0 & a & a\end{array}=-1.0$ & & \\
\hline $144000016=$ odd_digit & & \\
\hline 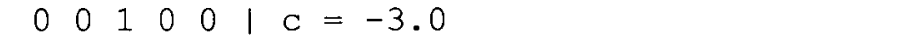 & & \\
\hline $\begin{array}{lllllll}1 & 0 & 0 & 2 & 0 & \mathrm{~d} & \mathrm{~d}=\mathrm{S}[\mathrm{rlen}-1]\end{array}$ & & \\
\hline $\begin{array}{lllllll}0 & 0 & 0 & 0 & 2 & 1 & e=-2.0\end{array}$ & & \\
\hline
\end{tabular}

Figure 19 Student C's TS + C's CP - Iteration 1

We first add the missing category to the student's CP (the same category as for student A). Once the abstract test cases are (automatically) re-created from the updated $\mathrm{CP}$, the execution of $\mathrm{C} 4.5$ produces the decision tree of Figure 20 with nine rules. The decision tree shows a missing choice (\#RLENCHARS=Even) which is due to missing test cases (Case 2.2). Using the heuristic described in section 4.4 for adding choice combinations, the tree also suggests that the following six combinations of choices are potentially missing, among which only the two identified with a '*' are not already exercised by the test suite: 


\begin{tabular}{|c|c|}
\hline $\begin{array}{l}\text { ODD DIGIT }=\text { odd digit }=-1 \\
\text { \#RLENCHARS }=\text { zero: } \\
\mid \text { | RLEN }=\text { rlen }=0: \\
\text { | RLEN }=\text { rlen }=[1 \ldots \text { Slength }]: \\
\text { \#RLENCHARS }=\text { Even } \\
\text { | RLEN }=\text { rlen }=[1 \ldots \text { Slength }]:\end{array}$ & $\begin{array}{l}\text { ODD DIGIT }=\text { odd digit }=[0 \ldots 15] \\
\text { \#RLENCHARS }=\text { Zero: } \\
\mid \text { I RLEN }=\text { rlen }=0: \\
\mid \text { RLEN }=\text { rlen }=[1 \ldots \text { Slength }]: \\
\text { \#RLENCHARS }=\text { Even } \\
\text { | RLEN }=\text { rlen }=[1 \ldots \text { Slength }]:\end{array}$ \\
\hline
\end{tabular}

The tree also shows two rules (rules 2 and 6) with no instances: these are unfeasible combinations of choices (Case 3.1), because if the number of first hexadecimal characters in string $S$ is odd (\#RLENCHARS=Odd) then it is not possible for $r$ len to be zero $($ RLEN $=0)$.

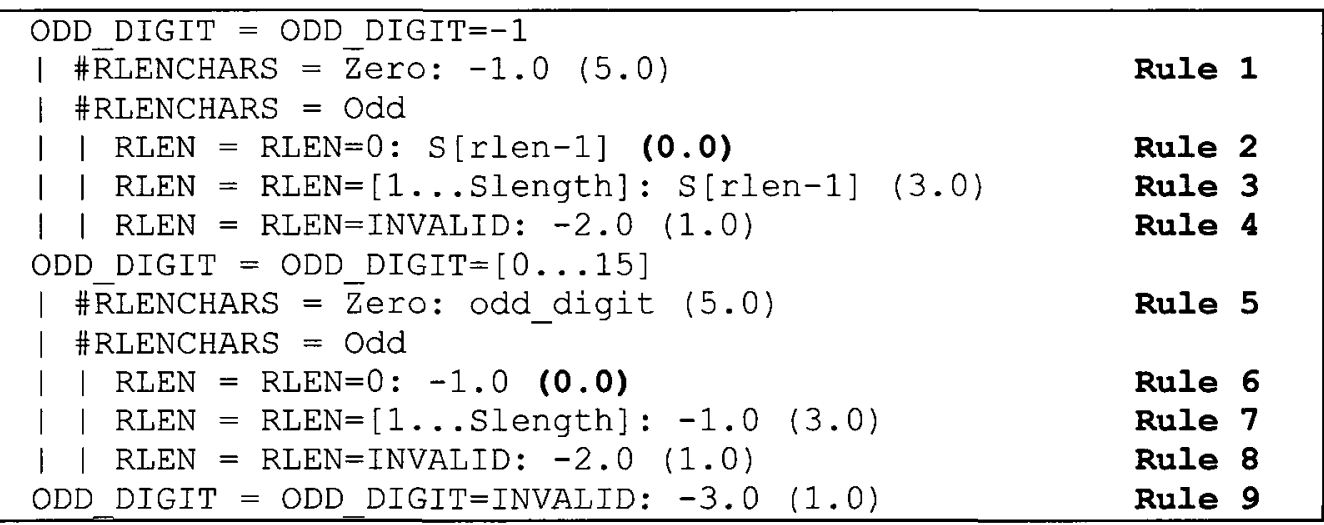

\section{Figure 20 Student C's TS + C's CP - Iteration 2}

We therefore create two test cases, for the two missing combinations, (automatically) produce the corresponding abstract test cases and re-run $\mathrm{C} 4.5$, which returns the decision tree in Figure 21 with 13 rules. This tree shows three rules (rules 6, 9, and 12) with no instances: these rules are feasible but involve an error condition (odd_digit=INVALID) which already appears in another rule (rule 3). Following the heuristic described in Section 4.4, there are no combinations of choices missing in the decision tree. The tree suggests redundancy, since rules $1,2,4,5,7,8$, and 13 cover more than one instance. 


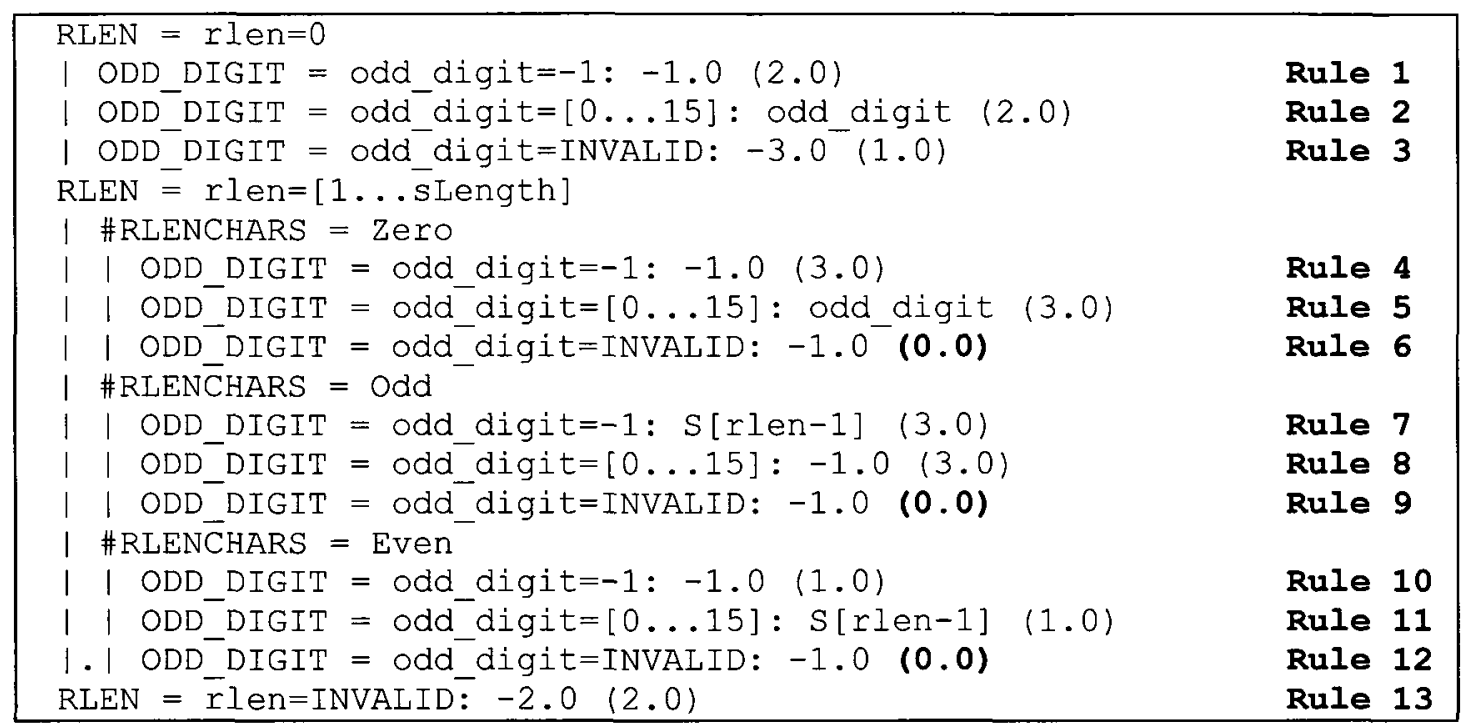

Figure 21 Student C's TS + C's CP - Iteration 3

We remove redundant test cases and obtain the tree in Figure 22 with 13 rules. No occurrence of the problems discussed in section 4.2 can be found and the iterative process of Figure 4 stops. The final $\mathrm{CP}$, which contains five categories and 14 choices of which categories 1,2 , and 5 (i.e., three categories and nine choices) are used in the tree, is equivalent to the Expert $\mathrm{CP}$ (Section 5.4.1).

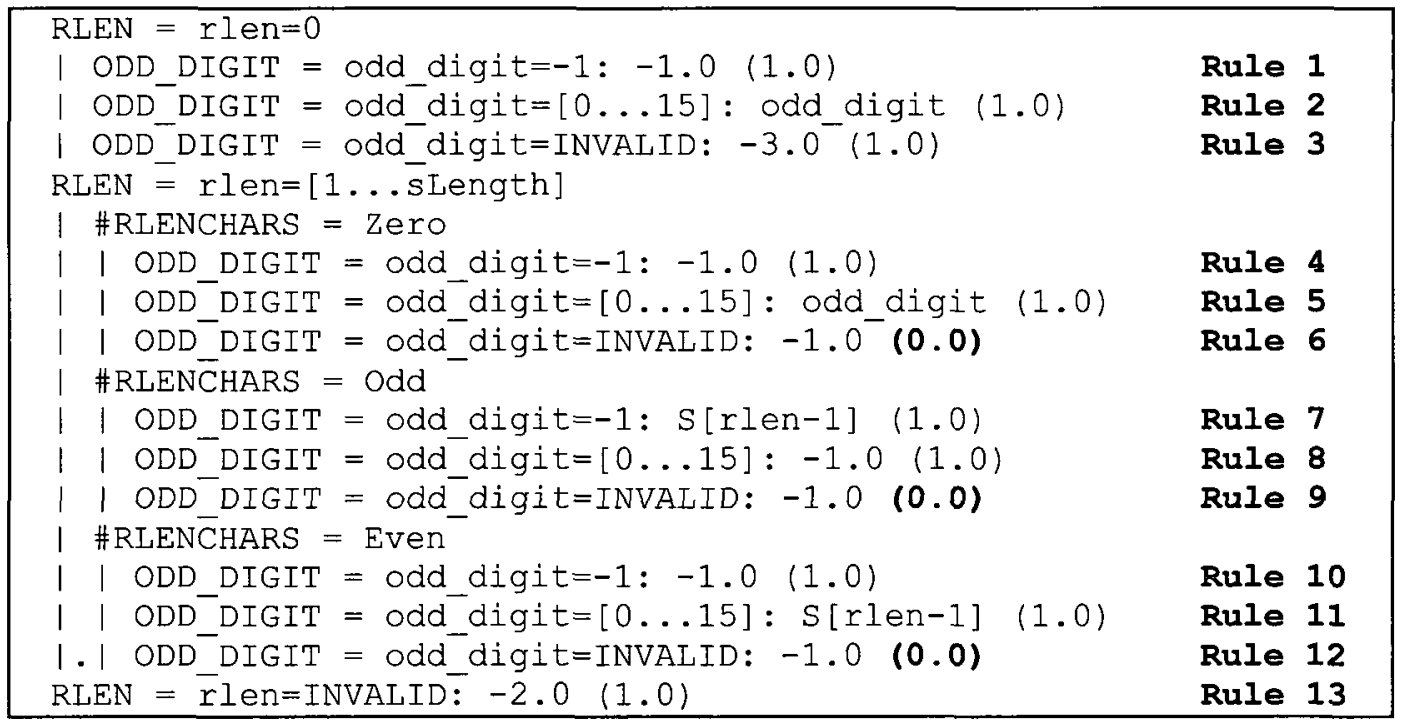

Figure 22 Student C's TS + C's CP - Final Tree 


\subsection{Results with the Expert CP - OSS Context}

Note that since we use our $\mathrm{CP}$ to classify the student test cases we have $\mathrm{CP}=\mathrm{CP}^{\prime}$ in our definition of category partition specification comparison (Section 5.4.1. We still compare $C P_{D T 1}$ and $C P_{D T 2}$ using the definition in section 5.4.

\subsubsection{Student X's TS with the Expert CP}

After creating abstract test cases for student X's test suite (15 test cases) using the Expert $\mathrm{CP}$, and running C4.5, we obtain the decision tree in Figure 23 with five rules. The tree shows no misclassified instance (test case). This is expected as we use the Expert CP: a complete set of categories and well-defined choices should result into correct classifications. However, the tree shows a number of issues: (1) not all the categories of the Expert CP appear in the tree (Case 2), specifically, categories 5, 7,8, 9, 10, and 11 are missing (Appendix A.2); (2) some choices (in the remaining, used categories) are missing (Case 2), specifically choices C3, C7, C8, C9, and C14 (Appendix A.2); (3) There are missing combinations of choices (Case 3), specifically, using the heuristic described in Section 4.4 for adding combinations, the tree suggests that the following 16 combinations of choices are potentially missing, among which two (identified with $a^{(* \prime}$ ) are already exercised by the test suite: 

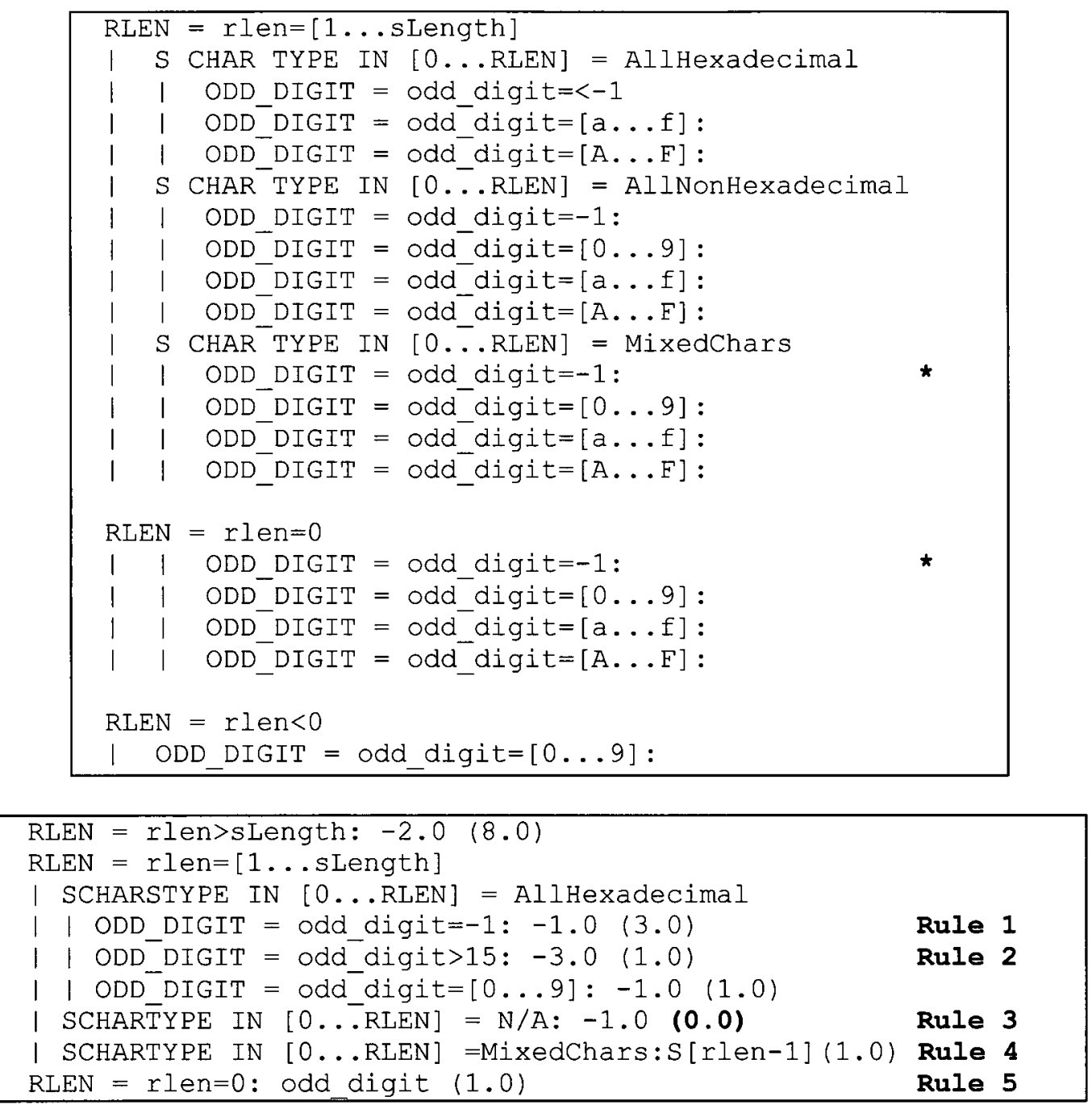

Figure 23 X's TS + Expert CP - Iteration 1

This initial use of $\mathrm{C} 4.5$ therefore allows us to improve the test suite. Fourteen new test cases are created and transformed into abstract test cases, and C4.5 is re-executed with a total of 29 abstract test cases (15 initial ones and 14 new ones). The result is the decision tree of Figure 24 with 28 rules. This tree shows potentially missing choice combinations $((0.0)$ in bold face-rules $3,5,8,12,13,17,19,23$, and 25): rules 8,12 , and 13 are feasible and cover no test case, rules $3,5,17,19,23$, and 25 are combinations of choices that are feasible but involve an error condition which already appears in another rule (rules 9 and 11). The tree also suggests the following 
19 missing combinations of choices, among which eleven (identified with $a^{(* ')}$ are not already covered by the test suite:

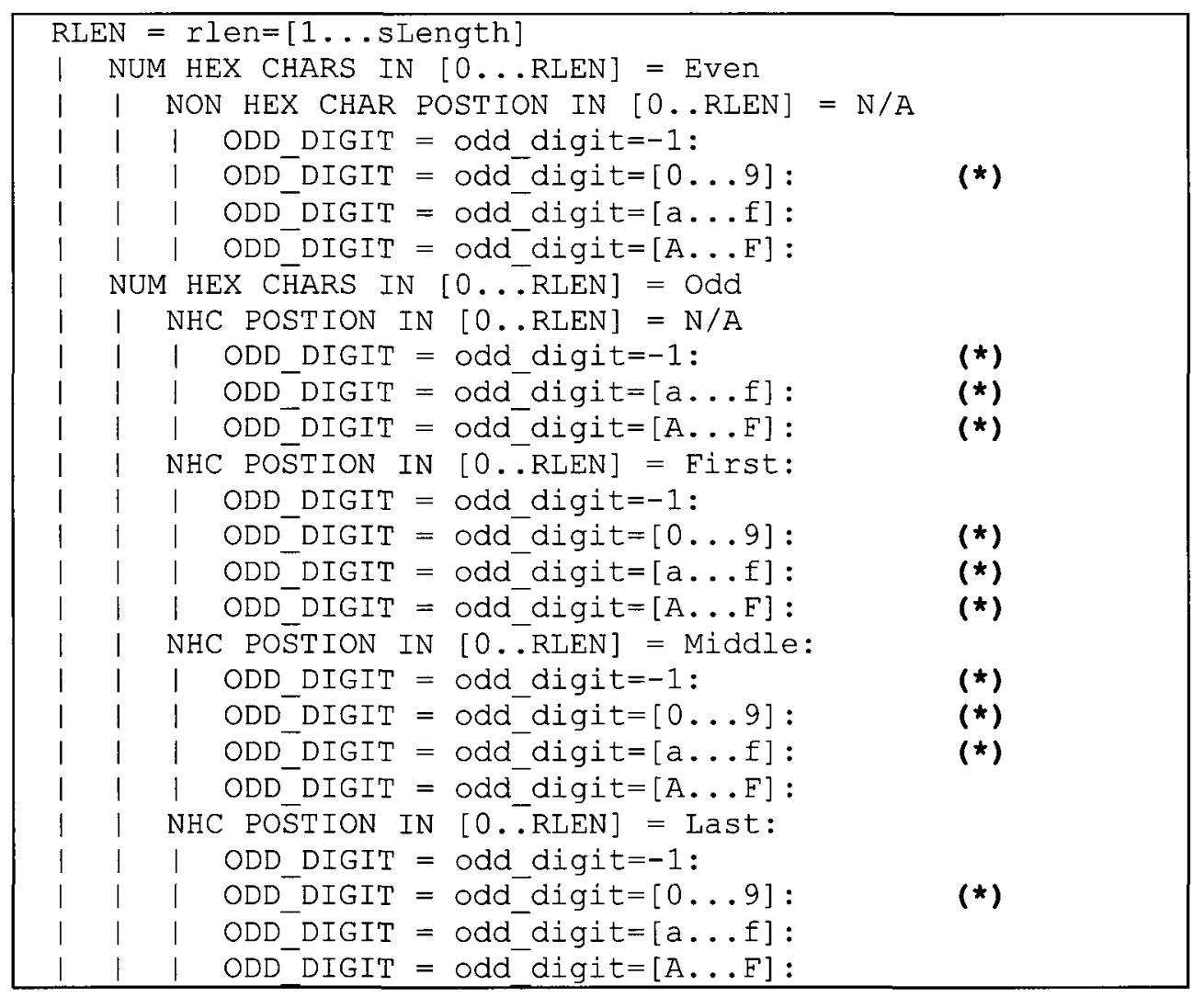




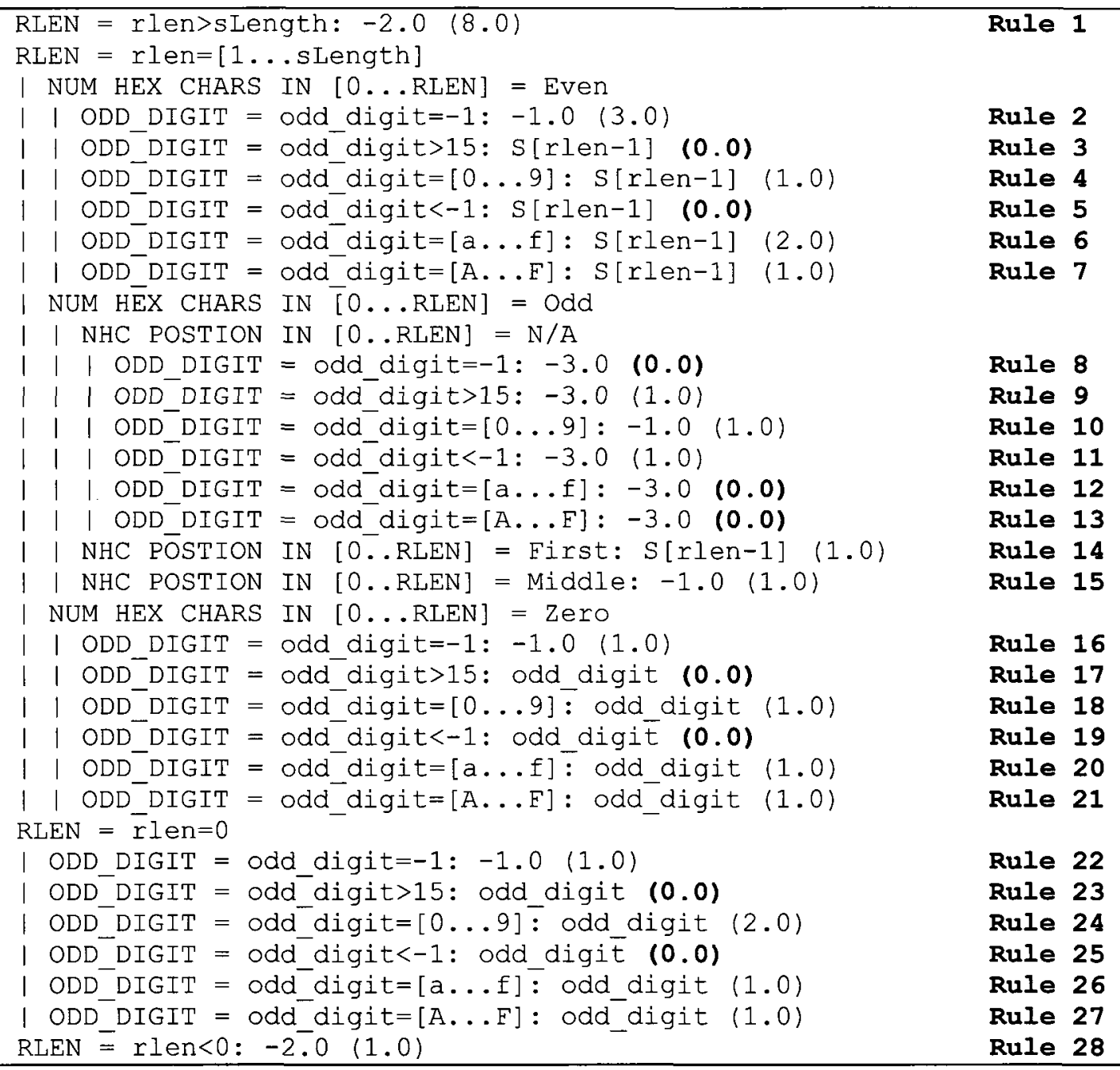

Figure 24 X's TS + Expert CP - Iteration 2

After having added eleven test cases, automatically produced the corresponding abstract test cases and re-run C4.5, we obtain the tree in Figure 25 with 26 rules. Rules $3,5,15,17,21$, and 23 are combinations of choices that are feasible but involve error conditions which already appear in rules 9 and 11: there is no need to add test cases. Some rules in Figure 25 contain a large number of instances (test cases): e.g. rule 1 . According to the decision tree, these test cases trigger the same input-output relationship in the program. 


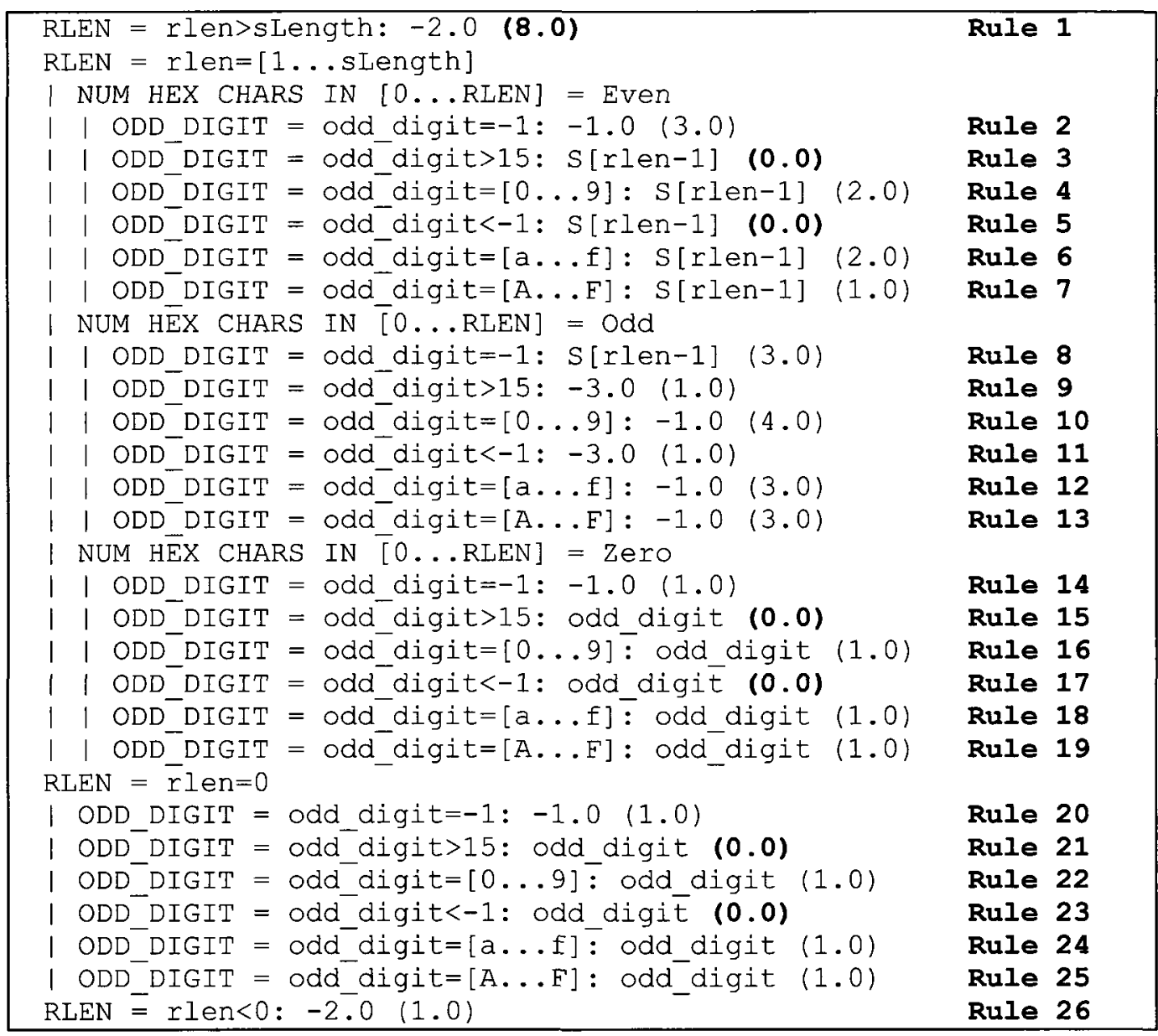

Figure 25 X's TS + Expert CP - Iteration 3

We remove some of those test cases (randomly selected) and only keep one of them per rule unless code coverage is affected. We obtain a test suite of 20 test cases. The tree in Figure 26 with 26 rules is similar to the one in Figure 25 except that the number of instances for all rules becomes 1 . No occurrence of the problems discussed in Section 4.2 can be found and the iterative process of Figure 4 stops. It is worth mentioning that the decision tree we obtain with student X's TS and Expert CP uses the same categories and choices as when using the Expert's CP and the Expert's test suite together (Appendix C.1): the two CPs are therefore equivalent. 


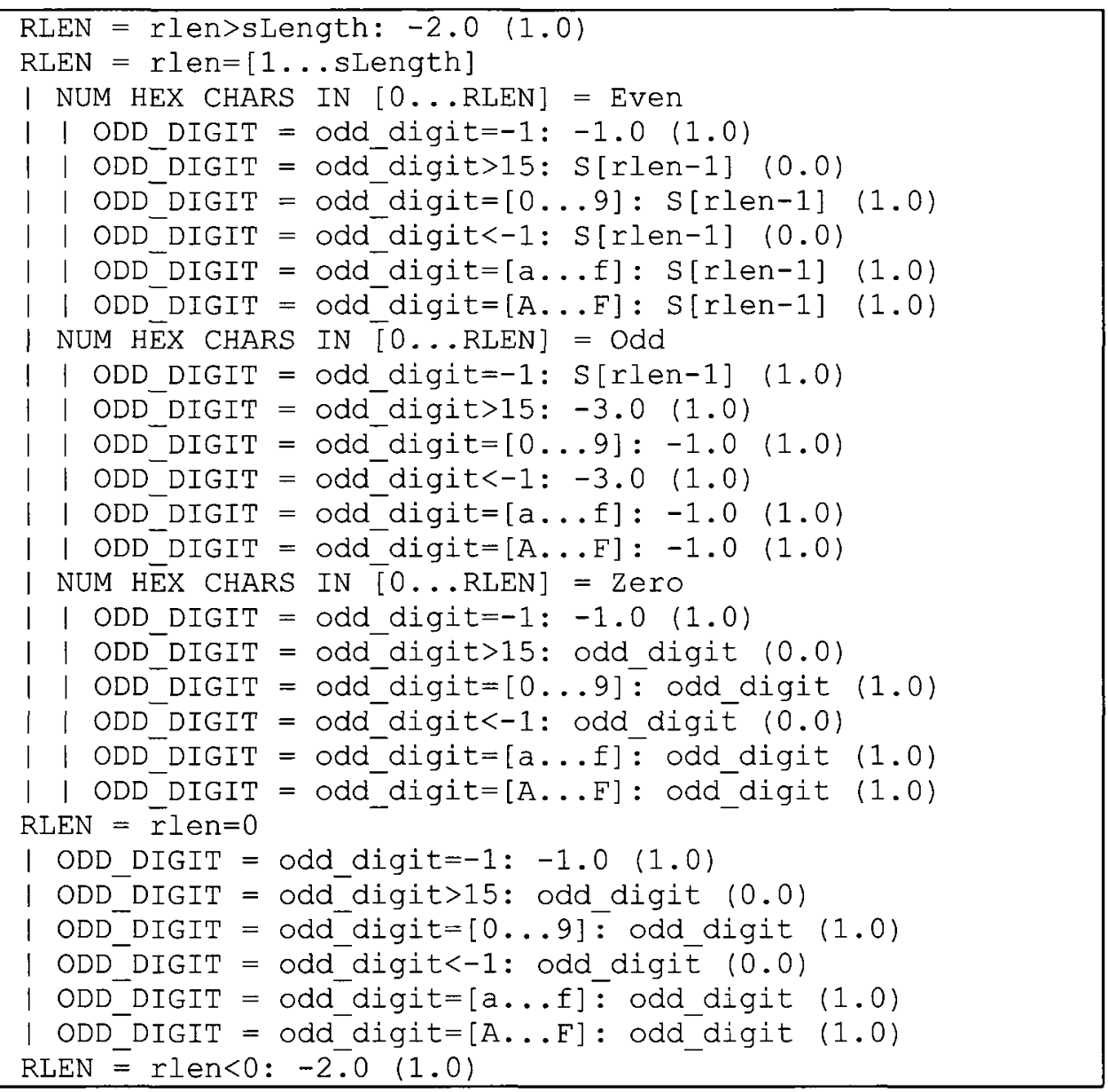

Figure 26 X's TS + Expert CP - Final Tree

\subsubsection{Student Y's TS with the Expert CP}

Student Y's test suite contains 20 test cases. The corresponding abstract test cases are used as inputs to $\mathrm{C} 4.5$ which generates the decision tree in Figure 27 with nine rules. Similarly to X's TS, the decision tree shows no misclassification but some categories are missing (Categories 5, 6, 7, 8, 10, and 11) and some choices of used categories are missing (specifically, C7 and c9): Case 2 in Section 4.2. The tree shows potentially missing choice combinations (rule 3): rule 3 is feasible and covers no test case. Using the heuristic described in Section 4.4 for adding choice combinations, the tree also 
suggests the following 15 missing combinations of choices, among which only three are already exercised by the test suite (identified with $\mathrm{a}^{\text {'*' }}$ in the list below):

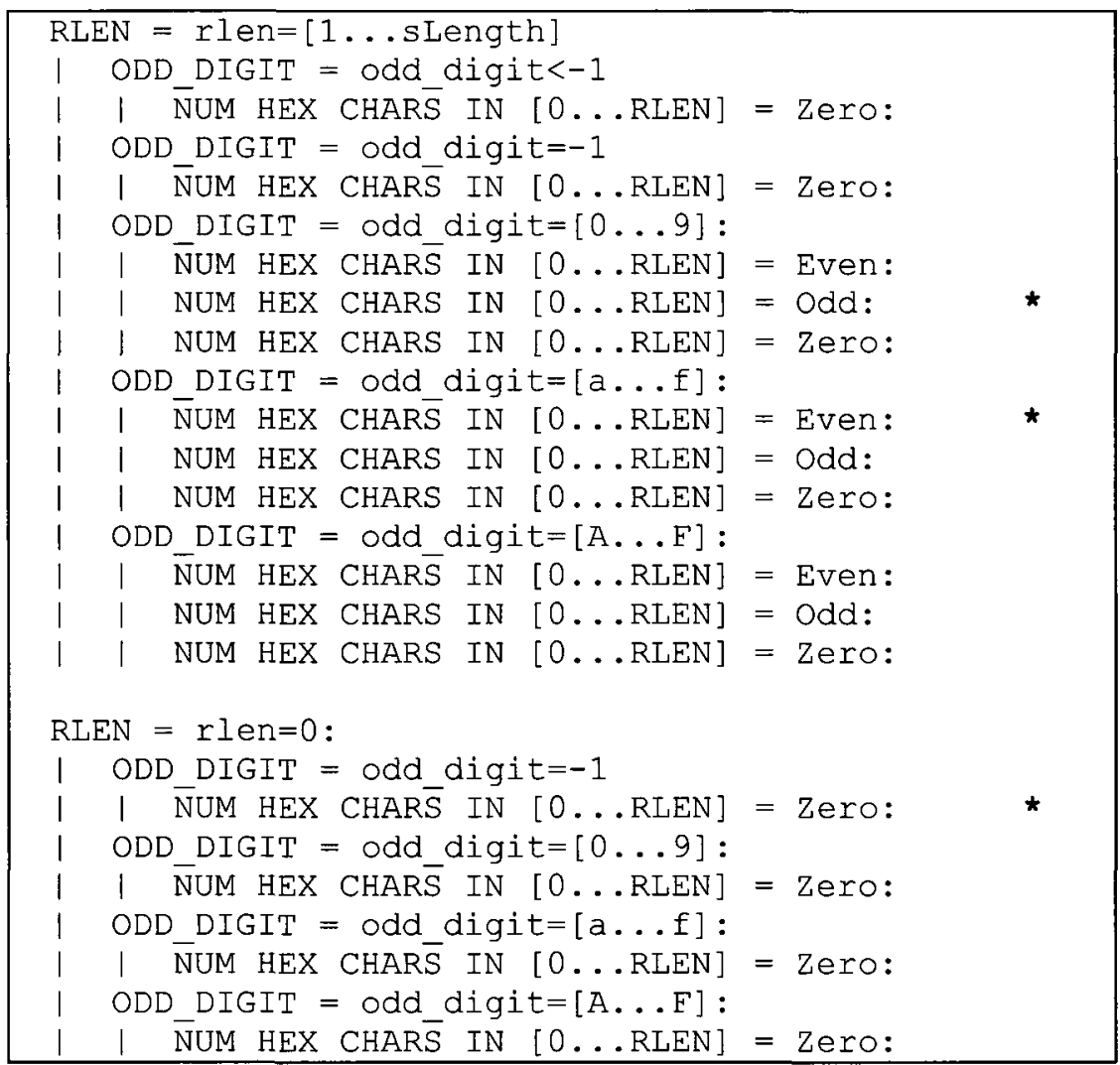

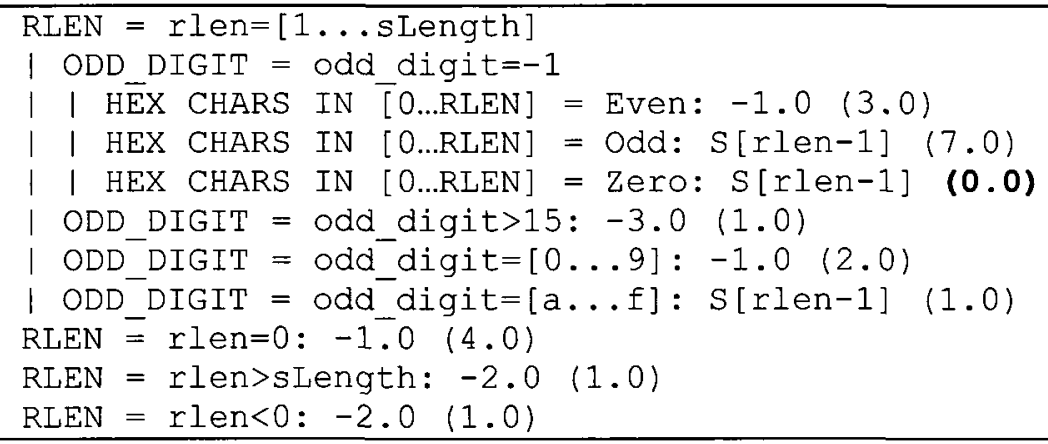

Figure 27 Y's TS + Expert CP - Iteration 1

This initial use of C4.5 therefore allows us to improve the test suite: Step 4 in Figure 4. 12 new test cases are transformed into abstract test cases, and C4.5 is re-executed with a total of 32 abstract test cases ( 20 original and 12 new ones). The result is the tree of Figure 28 with 26 rules. In this tree, rules $8,12,14,18,20$, and 24 are 
combinations of choices that are feasible but involve an error condition which already appears in rules 2 and 6 . Rule 10, on the other hand, is a feasible combination of choices that covers no test case.

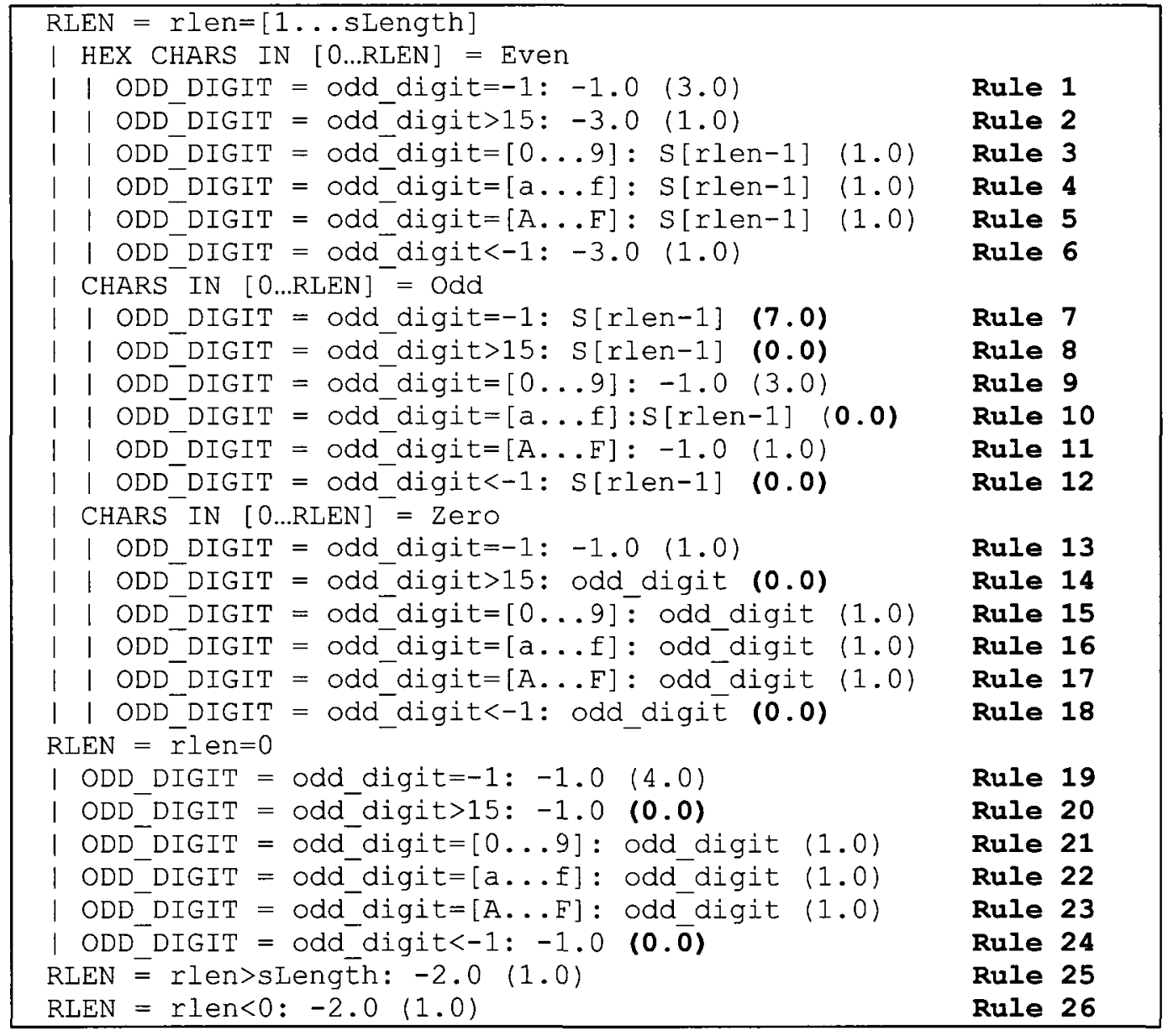

Figure 28 Y's TS + Expert CP - Iteration 2

We therefore create one new test case, (automatically) produce the corresponding abstract test case and re-run $\mathrm{C} 4.5$ with a total of 33 abstract test cases, which returns the decision tree in Figure 29 with 26 rules. The tree shows six rules with no instances (rules $8,12,14,18,20$, and 24): these are feasible combinations of choices but involves an error condition which already appears in rules 2 and 6 . Additionally, some rules in Figure 29 contain a large number of instances (test cases). 


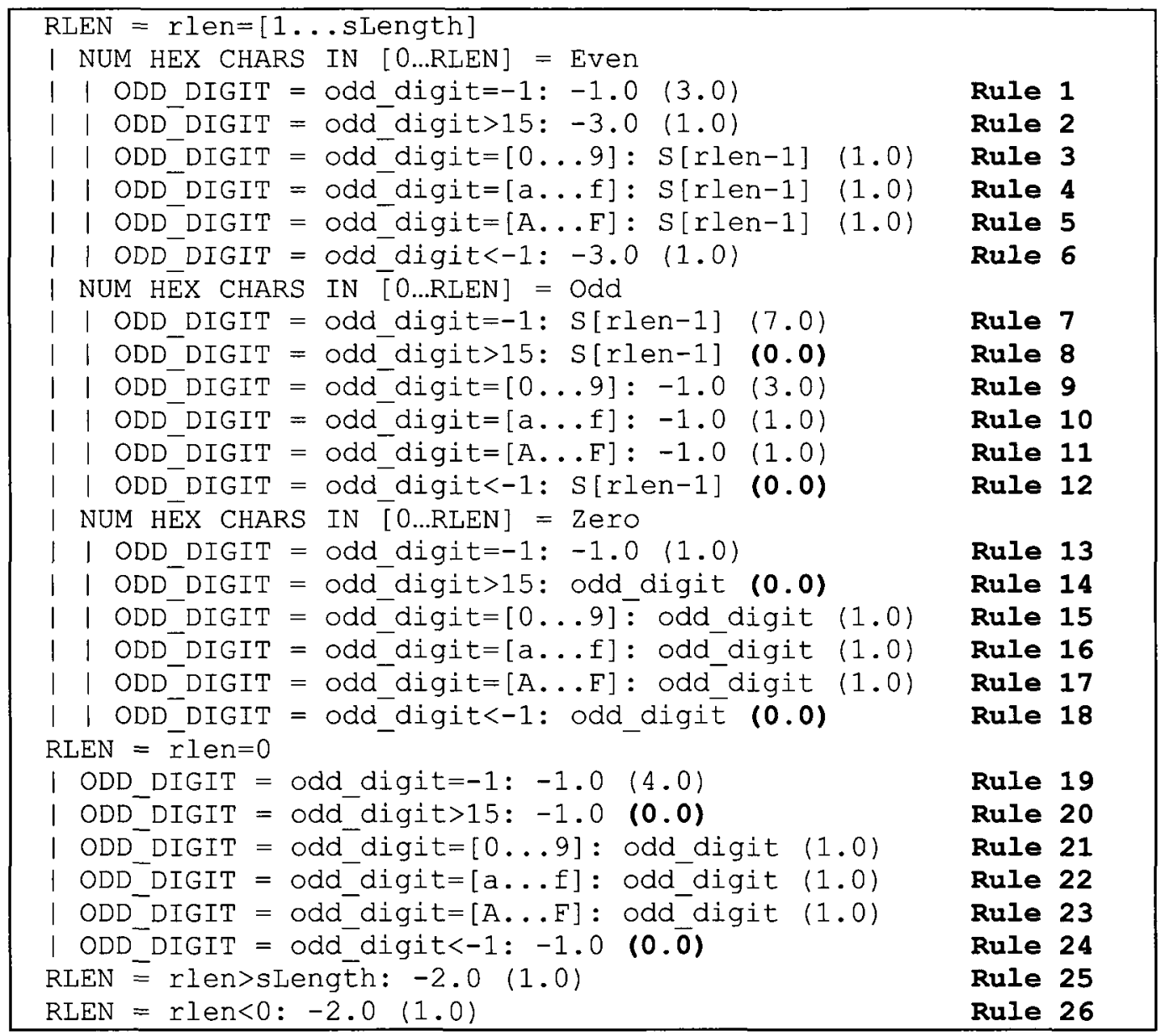

Figure 29 Y's TS + Expert CP - Iteration 3

We remove some of those test cases (randomly selected) and only keep one per rule without affecting the code coverage. We therefore obtain a test suite of 20 test cases, and a tree similar to the one in Figure 29 except that the number of instances for all feasible rules becomes one. No occurrence of the problems discussed in Section 4.2 can be found and the iterative process of Figure 4 stops. The decision tree we obtain with student Y's TS and Expert CP uses the same categories and choices as when using the Expert's CP and the Expert's test suite together (Appendix C.1): the CPs are equivalent. Keep also in mind that the test suite we obtained in Iteration 3 and the reduced test suite have the same code coverage. 


\subsubsection{Student Z's TS with the Expert CP}

Student Z's test suite contains eleven test cases. The corresponding abstract test cases are used as inputs to $\mathrm{C} 4.5$ which generates the decision tree in Figure 30 with 13 rules. Similarly to X's DT, the decision tree shows no misclassification but some categories are missing (Categories 5, 6, 7, 9, 10, an 11 in Appendix A.2) and some choices of used categories are missing (specifically, c7): Case 2. This tree shows potentially missing choice combinations (rules $4,5,6$, and 7 ): these are four feasible rules that cover no test case. Using the heuristic described in Section 4.4 for adding choice combinations, the tree also suggests the following 36 missing combinations of choices, among which four combinations (identified with $a^{(* *)}$ ) are already exercised

by the test suite:

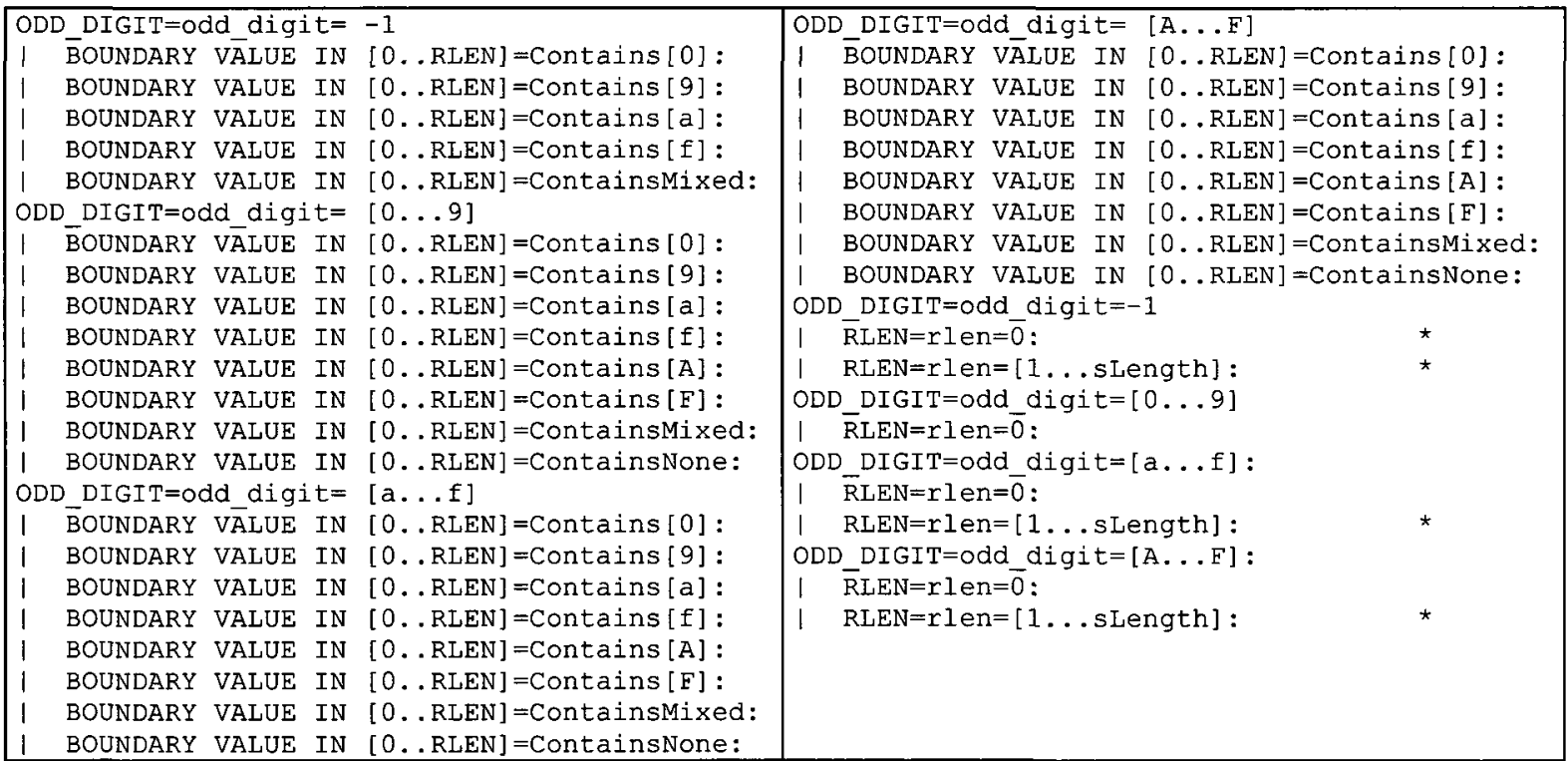




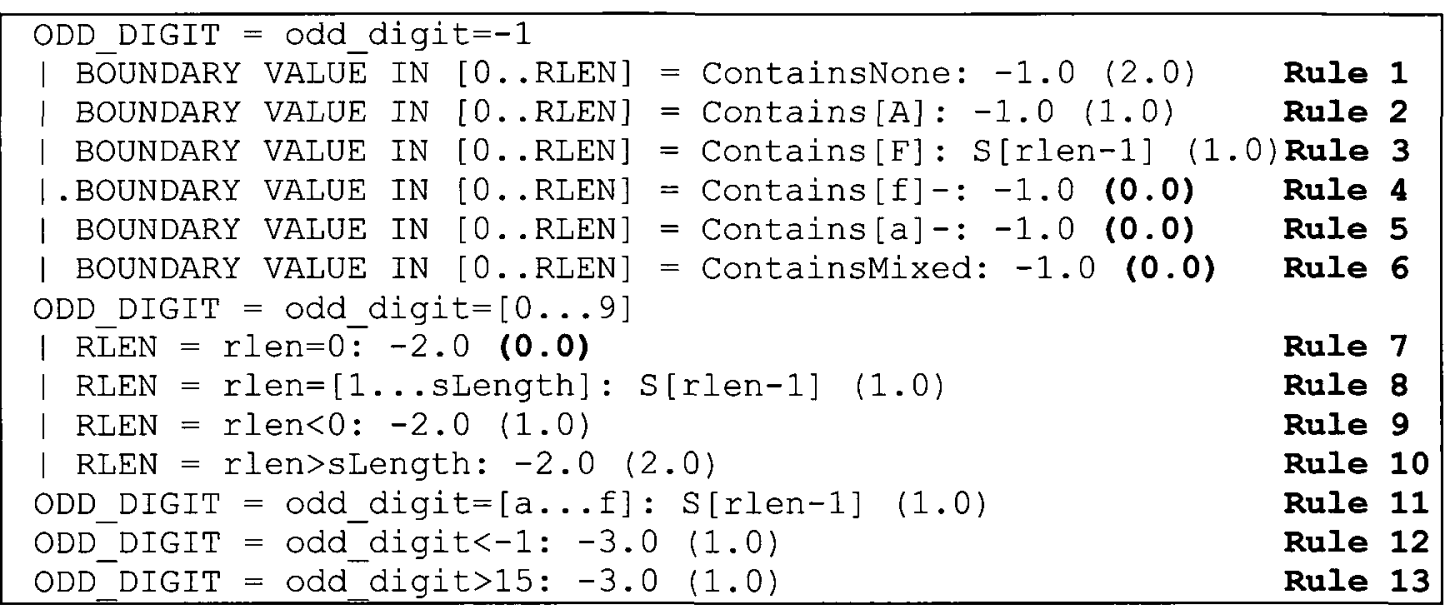

Figure 30 Z's TS + Expert CP - Iteration 1

We refine the test suite by adding 32 new test cases. They are transformed into abstract test cases, and C4.5 is re-executed with a total of 43 abstract test cases (11 original and 32 new ones). The result is the tree of Figure 31 with 21 rules. This tree shows potentially missing choice combinations (rules, 4, 5, 11, 17, and 18): rules 5 and 18 are feasible combinations of choices but involve an error condition which already appears in rule 12 . Rules 4,11 , and 17 are also feasible combinations of choices and involve the same error condition (odd_digit<-1) for which there is no test case. In the end, using our heuristic, the tree suggests the following five missing combinations of choices of which none is exercised by the test suite:

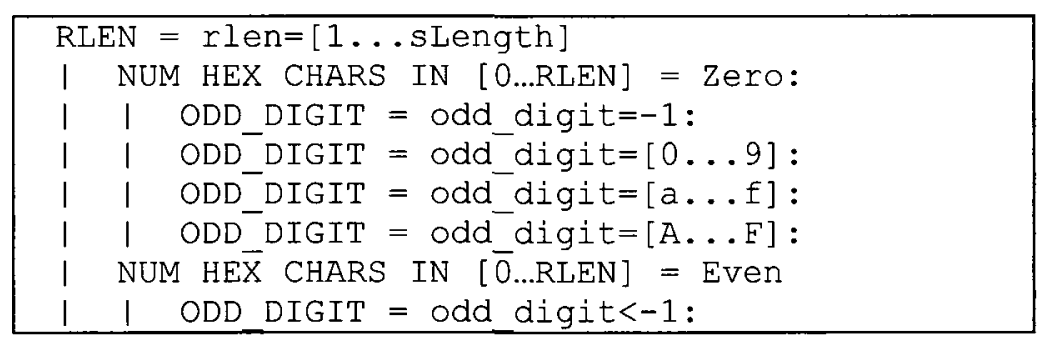




\begin{tabular}{|c|c|c|}
\hline \multicolumn{3}{|l|}{ RLEN $=r l e n=0$} \\
\hline | ODD_DIGIT $=$ odd_digit $=-1:-1.0(1.0)$ & Rule & \\
\hline | $\mathrm{ODD}^{-} \mathrm{DIGIT}=\mathrm{odd}^{-}$digit $=[0 \ldots 9]:$ odd digit & Rule & 2 \\
\hline | ODD_DIGIT = odd digit=[a...f] : odd_digit (1.0) & Rule & 3 \\
\hline | ODD DIGIT = odd digit<-1: odd digit $(0.0)$ & Rule & 4 \\
\hline | ODD_DIGIT $=$ odd_digit $>15$ : odd_digit $(0.0)$ & Rule & 5 \\
\hline | ODD_DIGIT $=$ odd_digit $=[\mathrm{A} . . . \mathrm{F}] \overline{\mathrm{l}}$ odd_digit $(1.0)$ & Rule & 6 \\
\hline \multicolumn{3}{|l|}{ RLEN $=$ rlen=[1... sLength $]$} \\
\hline I NUM HEX CHARS IN $[0 \ldots$ RLEN $]=$ Zero: $-3.0(1.0)$ & Rule & 7 \\
\hline I NUM HEX CHARS IN $[0 \ldots$ RLEN $]=$ Even & & \\
\hline I $\mid$ ODD_DIGIT $=$ odd_digit=-1: $-1.0(4.0)$ & Rule & 8 \\
\hline $1 \mathrm{ODD}^{-}$DIGIT $=$odd $^{-}$digit $=[0 \ldots 9]: \mathrm{S}[\mathrm{rlen}-1]$ & Rule & 9 \\
\hline $1 \mid$ ODD DIGIT $^{-}$odd digit $=[a \ldots f]: S[r l e n-1]$ & Rule & 10 \\
\hline | $\mid$ ODD_DIGIT = odd_digit<-1: S[rlen-1] (0.0) & Rule & 11 \\
\hline | $\mid$ ODD_DIGIT = odd_digit>15: $-3.0(1.0)$ & Rule & 12 \\
\hline $\mid$ ODD_DIGIT $=$ odd_digit $=[A \ldots F]: S[r l e n-1]$ (6.0) & Rule & 13 \\
\hline \multicolumn{3}{|l|}{ I NUM HEX CHARS IN $[0 \ldots$ RLEN $]=$ Odd } \\
\hline | | ODD_DIGIT = odd_digit=-1: S[rlen-1] (4.0) & Rule & 14 \\
\hline|| ODD $^{-}$DIGIT $=$odd $^{-}$digit $=[0 \ldots 9]:-1.0(1.0)$ & Rule & 15 \\
\hline | $\mid$ ODD DIGIT = odd digit=[a...f] $:-1.0$ (2.0) & Rule & 16 \\
\hline I $\mid$ ODD DIGIT = odd digit<-1: $-1.0 \quad(0.0)$ & Rule & 17 \\
\hline $1 \mid$ ODD DIGIT $^{-}$odd digit $>15:-1.0$ & Rule & 18 \\
\hline $\mid$ ODD_DIGIT $=$ odd_digit=[A...F] $:-1.0$ (2.0) & Rule & 19 \\
\hline RLEN $=\bar{r} l e n<0:-2.0(1.0)$ & Rule & 20 \\
\hline RLEN $=$ rlen>sLength: -2.0 & Rule & 21 \\
\hline
\end{tabular}

\section{Figure 31 Z's TS + Expert CP - Iteration 2}

We therefore create five test cases, (automatically) produce the corresponding abstract test cases and re-run $\mathrm{C} 4.5$ with a total of 48 abstract test cases (43 from Iteration 2 and five new ones) which returns the decision tree in Figure 32 with 26 rules. This tree shows five rules (rules $4,5,11,22$, and 23): these are feasible combinations of choices involving an error condition which already appears in rules 16 and 17. Some rules contain a large number of instances (test cases), e.g. rule 14. 


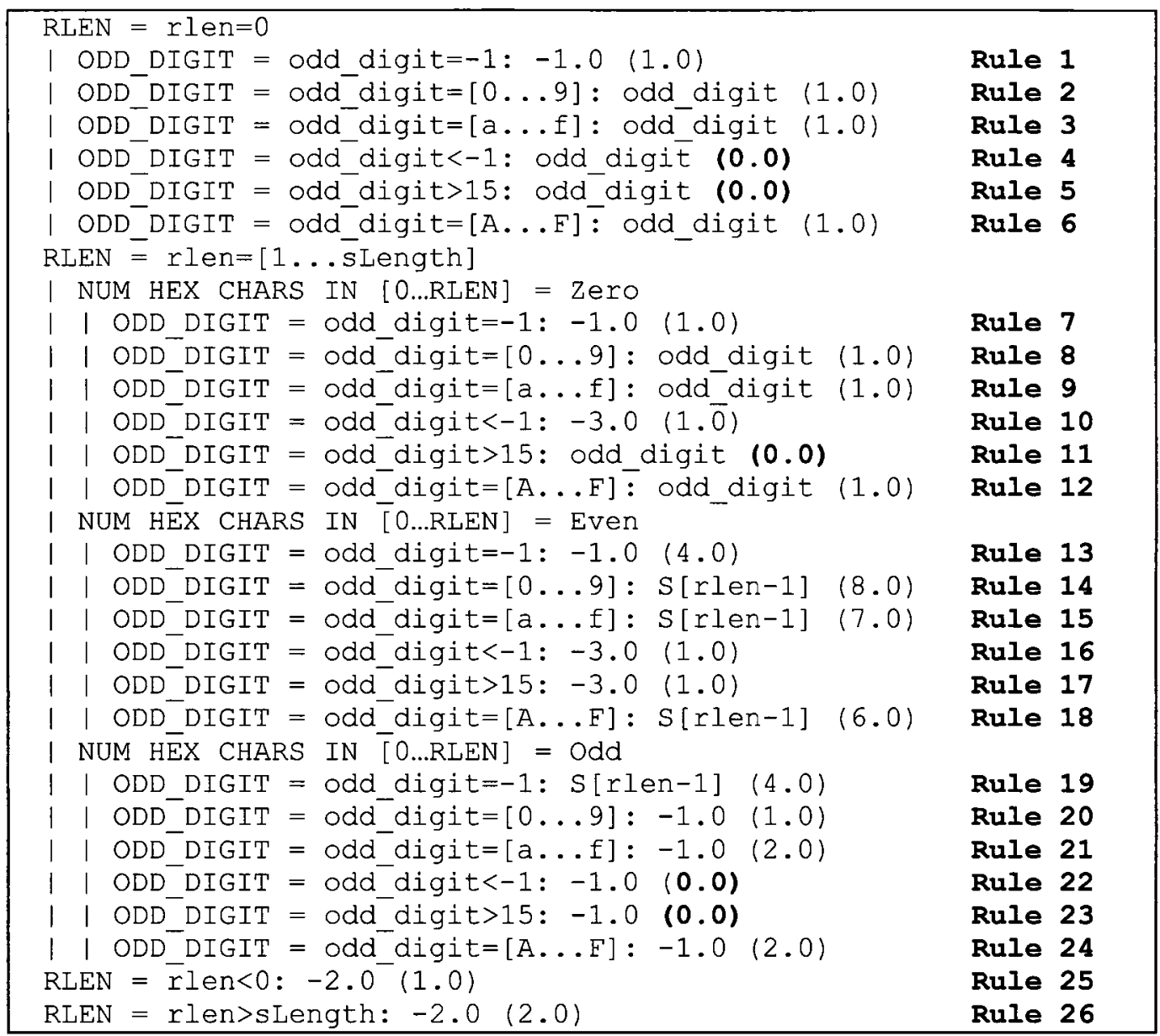

Figure 32 Z's TS + Expert CP - Iteration 3

We remove some of those test cases (randomly selected) and only keep one per rule unless code coverage is affected. We therefore obtain a test suite of 21 test cases, and a tree similar to the one in Figure 32 except that the number of instance for all feasible rules becomes one. No occurrence of the problems discussed in Section 4.2 can be found and the iterative process of Figure 4 stops. The decision tree we obtain with student Z's TS and Expert CP uses the same categories and choices as when using the Expert's CP and the Expert's test suite together (Appendix C.1): the CPs are equivalent. 


\subsection{Assessing the fault detection effectiveness of improved test suites}

To show the effectiveness of the improved test suites, we use method level mutation operators discussed in Section 5.7.1 using MuJava-a mutation system for java. The results are discussed in Section 5.7.2.

\subsubsection{Mutation Testing}

Mutation testing [30] is a fault based testing strategy that measures the quality/adequacy of test cases by examining whether they can reveal certain types of faults [31]. Mutation operators are applied to introduce single faults into the original program thereby creating a set of faulty versions, called mutants. Mutation operators describe syntactical changes to the program [32]. A mutant is said to be killed if a test set can differentiate a mutant from the original program, the mutant is called alive otherwise. A mutant may stay alive either because it is an equivalent mutant, i.e. it always produces the same output as the original program but is syntactically different, or the test suite is inadequate. A test suite that can kill all non-equivalent mutants is called an adequate test suite.

Our interest in using mutants is to assess the fault detection effectiveness of the improved test suites. Mutants have indeed been used to evaluate or compare test suites and testing techniques $[33,34]$.

MuJava [32], a mutation system for Java, is used to generate mutants for the PackHexChar program. A total of 254 mutants were generated of which 231 are non equivalent mutants (We consider a mutant as equivalent if it is not killed by the 
Expert TS). Figure 33 shows the number of mutants (equivalent and non equivalent) generated for each mutation operator. Note that there are no mutants for mutation operators COD, LOR, LOD, ASR, because the generated mutants were syntactically incorrect. MuJava uses both method and class-level mutation operators. Method-level mutation operators modify expression by replacing, deleting, and inserting primitive operators, whereas class-level mutation operators handle object-oriented specific features such as inheritance, polymorphism and dynamic binding [32]. We are interested in method-level mutation operators that modify expressions by replacing, deleting, and inserting primitive operators. (The PackHexChar program only has one class, therefore operators targeting inheritance, polymorphism ... are not adequate.) Table 2 lists all the method level operators implemented in MuJava. They are all used to generate mutants for the case study:

\begin{tabular}{|l|l|}
\hline Operator & Description \\
\hline AOR & Arithmetic Operator Replacement \\
\hline AOD & Arithmetic operator Deletion \\
\hline AOI & Arithmetic operator Insertion \\
\hline ROR & Relational Operator Replacement \\
\hline COR & Conditional Operator Replacement \\
\hline COD & Conditional operator Deletion \\
\hline COI & Conditional operator Insertion \\
\hline SOR & Shift Operator Replacement \\
\hline LOR & Logical Operator Replacement \\
\hline LOD & Logical operator Deletion \\
\hline LOI & Logical operator Insertion \\
\hline ASR & Assignment Operator Replacement \\
\hline
\end{tabular}

Table 2 Method-Level Mutation Operators for Java. 


\section{Number of Mutants for each Mutation Operator}

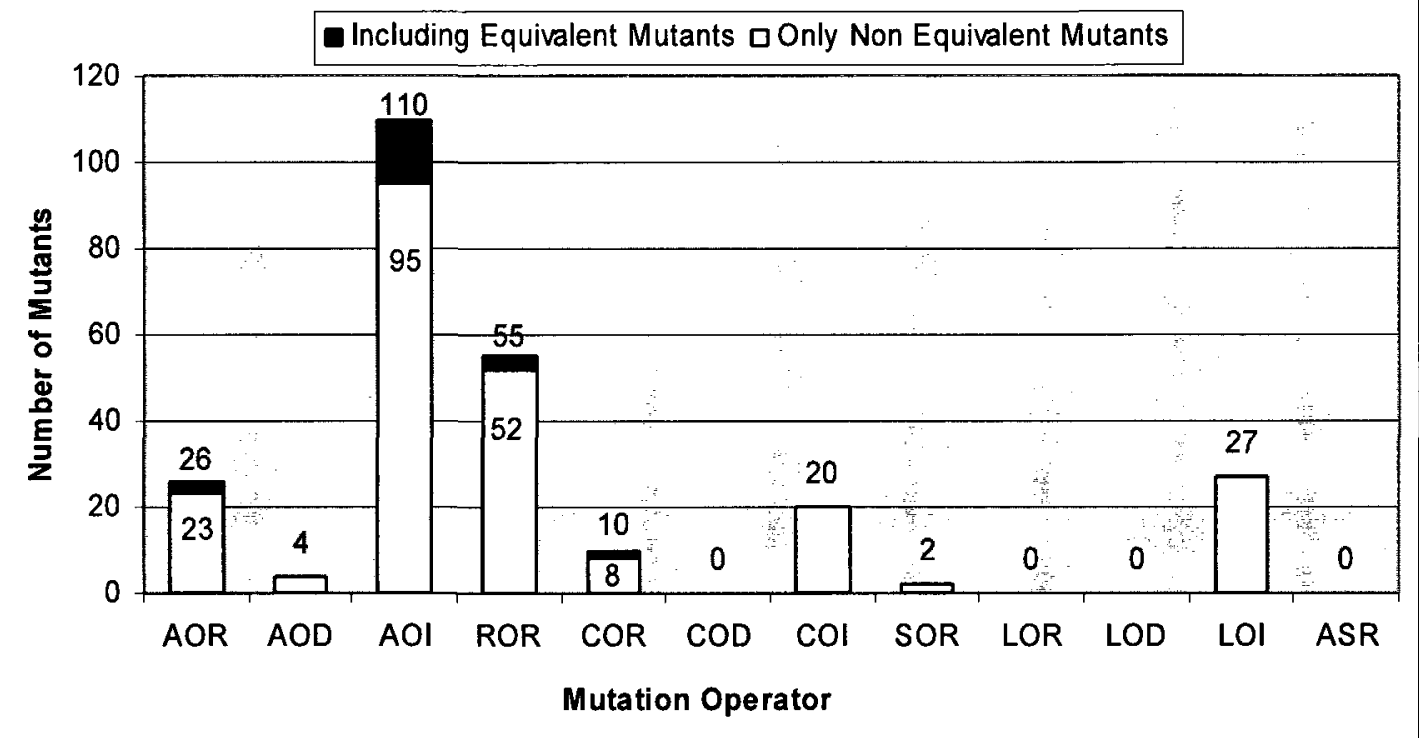

Figure 33 Number of (non-) Equivalent Mutants for each Mutation Operator

To show whether the improved test suites kill more mutants than the original test suite, we use mutated versions i.e. faulty version of the PackHexChar program, we then compare the size of the initial, improved and reduced test suites together with the number of mutants each one kills. We also investigate reasons why mutants remain alive by conducting a qualitative analysis.

\subsubsection{Results}

Table 3 shows the students ID and Iteration number (iteration 1 is the original student test suite), the size of the test suite (i.e., number of test cases), the number of mutants killed by the test suite (with percentage) and the coverage achieved by the test suite -

Recall from Section 5.3 that we use statement coverage. (The exact mutants not killed by the different students test suites can be found in Table 6, Appendix D.) 
First we can note differences between students initial test suites. Though the students all applied category partition from the same specification description, during the same amount of time, results show varying numbers of test cases with varying effectivenesses. Effectiveness varies between $75.3 \%$ (student X) and $97.8 \%$ (student Z), and size varies between 11 (student $Z$ ) and 31 (student $A$ ). Student $Z$ test suite was very cost effective, whereas Students B and C test suites are the worst.

\begin{tabular}{|c|c|c|c|c|c|}
\hline Student ID & Test Suite ID & Test Suite Size & Mutants Detected & $\%$ Of Mutants Detected & $\begin{array}{c}\% \text { Of Statement } \\
\text { Coverage }\end{array}$ \\
\hline \multirow{4}{*}{$X$} & Iteration 1 & 15 & $174 / 231$ & $75.3 \%$ & $88 \%$ \\
\hline & Iteration 2 & 29 & $224 / 231$ & $97 \%$ & $100 \%$ \\
\hline & Iteration 3 & 40 & $227 / 231$ & $98.3 \%$ & $100 \%$ \\
\hline & Iteration 4 & 20 & $225 / 231$ & $97.4 \%$ & $100 \%$ \\
\hline \multirow{4}{*}{$\mathrm{Y}$} & Iteration 1 & 20 & $225 / 231$ & $97.4 \%$ & $100 \%$ \\
\hline & Iteration 2 & 32 & $227 / 231$ & $98.3 \%$ & $100 \%$ \\
\hline & Iteration 3 & 33 & $227 / 231$ & $98.3 \%$ & $100 \%$ \\
\hline & Iteration 4 & 20 & $226 / 231$ & $97.8 \%$ & $100 \%$ \\
\hline \multirow{4}{*}{$\mathrm{Z}$} & Iteration 1 & 11 & $226 / 231$ & $97.8 \%$ & $100 \%$ \\
\hline & Iteration 2 & $\overline{43}$ & $230 / 231$ & $99.6 \%$ & $100 \%$ \\
\hline & Iteration 3 & 48 & $230 / 231$ & $99.6 \%$ & $100 \%$ \\
\hline & Iteration 4 & 21 & $230 / 231$ & $99.6 \%$ & $100 \%$ \\
\hline \multirow{3}{*}{ A } & Iteration 1 & 20 & $225 / 231$ & $97.4 \%$ & $100 \%$ \\
\hline & Iteration 2 & 26 & $226 / 231$ & $97.8 \%$ & $100 \%$ \\
\hline & Iteration 3 & 11 & $222 / 231$ & $96.1 \%$ & $100 \%$ \\
\hline \multirow{3}{*}{ B } & Iteration 1 & 31 & $200 / 231$ & $86.6 \%$ & $94 \%$ \\
\hline & Iteration 2 & 39 & $207 / 231$ & $89.6 \%$ & $95 \%$ \\
\hline & Iteration 3 & 12 & $205 / 231$ & $88.7 \%$ & $95 \%$ \\
\hline \multirow{3}{*}{$\mathrm{C}$} & Iteration 1 & 19 & $185 / 231$ & $80.1 \%$ & $90 \%$ \\
\hline & Iteration 2 & 21 & $200 / 231$ & $86.6 \%$ & $94 \%$ \\
\hline & Iteration 3 & 10 & $200 / 231$ & $86.6 \%$ & $94 \%$ \\
\hline \multirow{2}{*}{ Our $\mathrm{CP}+\mathrm{TS}$} & Iteration 1 & 221 & $231 / 231$ & $100 \%$ & $100 \%$ \\
\hline & Iteration 2 & 21 & $223 / 231$ & $96.5 \%$ & $100 \%$ \\
\hline
\end{tabular}

Table 3 Mutants killed and coverage by each test suite

Student X's original test suite (TS) contains 15 test cases which kill $75.3 \%$ of the mutants. After having improved the test suite in the second and third iterations with a test suite of 29 (resp. 40) test cases, 97\% (resp. 98.3\%) of the mutants are killed, which is quite an improvement, compared to the initial 75.3\%. Four mutants (ROR11, ROR18, ROR21, and ROR28) remain alive though, which is further discussed in Section 5.8. After having reduced student X's TS to 20 test cases, the number of 
mutants killed drops to $225(97.4 \%)$. By removing $50 \%$ of the test cases, the number of killed mutants drops only by two. The two mutants that are not killed by the reduced test suite are AOIU3 and AORB14 (see descriptions in Table 7, Appendix D). In order to kill AOIU3, a test case must map to the following choices of the Expert $\mathrm{CP}$ where feasible: $\mathrm{C} 1$, any choice in category $3, \mathrm{C} 12, \mathrm{C} 13$ or $\mathrm{C} 14, \mathrm{C} 17$ or $\mathrm{C} 18$, any choice in category $8, \mathrm{C} 28$ or $\mathrm{C} 29$, any choice in category 10 or 11 . For instance a test case with rlen $=3$, odd_digit $=-1$, and $S=" 0 a 1 "$, which maps to $C 2$, $\mathrm{C} 5, \mathrm{C} 12, \mathrm{C} 13, \mathrm{C} 19, \mathrm{C} 20$, and $\mathrm{C} 28$, will kill AOIU3. Note that the conditions to kill those mutants appear in the categories and choices of the Expert CP (which is used with student X's test suite-recall Section 5.6.1) but during (random) test suite reduction the test cases that killed those mutants where unluckily selected and removed, further discussion on why this is the case is discussed in Section 5.8.

Student Y's original TS kills $97.4 \%$ of the mutants. The test suite improvement process increases this value to $98.7 \%$ at iteration 2 (no further inmprovement at iteration 3). Four mutants (AOIS102, ROR8 ROR18, and ROR28) remain alive at the end of the improvement process, a discussion on why these mutants remain alive is given in Section 5.8. After having reduced the TS by 13 test cases, the percentage of mutants killed drops to $97.8 \%$ (two mutants survive), which is not a significant drop. The two mutants that are not killed after test suite reduction, shown in Table 8 (Appendix D), are for the same reason as for student X -see Section 5.8 for further discussion.

Student Z's original TS kills $97.8 \%$ of the mutants. The test suite improvement process increases this value to $99.6 \%$ at iteration 2: One mutant (AOIS102) remains 
alive. The reduced test suites kill $99.6 \%$ of the mutants as well. There is no such test case in the reduced test suite that will kill this mutant. The reason why this mutant stays alive is further discussed in Section 5.8.

Student A's original test suite kills $97.4 \%$ of the mutants. The test suite improvement process increases this value to $97.8 \%$. Four mutants (shown in Table 6) remain alive at the end of the improvement process, this is further discussed in Section 5.8. Student A's reduced test suite with eleven test cases kills $96.1 \%$ of the mutants. The four mutants that are not killed after test suite reduction are shown in Table 10 (Appendix D). The reason these mutants are not killed is because the test suite does not contain test cases that kill these mutant and we further discuss the reason in Section 5.8.

Student B's original test suite kills $86.6 \%$ of the mutatnts. The test suite improvement process increases this value to $89.6 \%$. Twenty four mutants (shown in Table 6) remain alive at the end of the improvement process, this is further discussed in Section 5.8. The reduced test suite with 12 test cases kills $88.7 \%$ of the mutants, which is slightly smaller than the number of mutants killed by the test suite before reduction $(89.6 \%)$. The twenty six mutants that are not killed after test suite reduction are shown in Table 11 (Appendix D).

Student C's original test suite kills $80.1 \%$ of the mutants. The test suite improvement process increases this value to $86.6 \%$. Thirty one mutants (shown in Table 6) remain alive at the end of the improvement process; this is further discussed in Section 5.8. The reduced test suite that contains ten test cases kills $86.6 \%$ of the mutants, which is 
the same as the test suite at the end of the improvement process, which contains 21 test cases.

Using expert CP and TS, all 231 mutants are killed (100\%), after having reduced the expert TS to 21 test cases ( $90 \%$ of reduction), the number of mutants killed drops to $96.5 \%$ ( 8 mutants less). The 8 mutants that are not killed after test suite reduction are shown in Table 12 (Appendix D). Recall that based on our test removal strategy (Section 4.4.2), test cases are randomly removed for each leaf (rule) unless the percentage of code coverage drops. We further discuss next our reduction strategy and why the number of alive mutant increases when test cases are removed.

The reason these mutants were not killed is because test cases satisfying some categories and choices related to the content of the input string s, i.e. not necessary to determine the ouput equivalence class, are not used in the tree and hence not used in the DT.

\subsection{Analysis of alive mutants after test suite improvement/reduction}

In Section 5.7.2, we noticed some issues with the improvement strategy as well the test suite reduction process. In this section, we explain why this happens and suggest a possible alternative.

\subsubsection{OSS Context}

We first discuss the context where we use a student TS with the Expert CP (OSS Context). Recall that in Section 4.4.1 two solutions are discussed for adding test cases The first is to follow the $\mathrm{CP}$ method and build all the feasible (according to properties 
and selectors) combinations of choices and select the ones that are missing in the abstract test suite and the second is to identify which combinations of choices may be relevant to determine the output class and could be missing from the test suite by using the decision tree. We decided to use the second solution: this heuristic is advantageous for it uses the information provided by the tree, when the tester intends to cover new choices, she does not have only the expensive option to exercise all the feasible combinations of choices, but can focus on combinations that are likely to affect the output. But by using the second solution when adding test cases we are potentially missing out some choices combinations that could potentially kill mutants that would otherwise not be killed by the first alternative since issing choice combinations would be identified not only based on the tree but also on the $\mathrm{CP}$ specification. Therefore, the number of mutants killed by using the first solution would be higher than the second solution. For instance, the main reason why mutants remain alive is that the test inputs do not contain boundary values ${ }^{9}$. In the case of Student X, four mutants (ROR11, ROR18, ROR21, ROR28) have not been killed by the improved test suite, and will only be killed if there are test cases where the first rlen characters in the input string contained an "a", "A", "f", or "F". Similarly, Student Y's improved test suite could have killed the mutants that involved boundary values (ROR8, ROR18, ROR28) if the first rlen characters in the input string contained 9, F, or f. By using the first solution, these mutants would have been killed.

\footnotetext{
${ }^{9}$ The input String may contains valid hex chars between [0...9], [a...f], and [A...F], where 0, 9, a, f, $\mathrm{A}$, and $\mathrm{F}$ are boundary values in the input string.
} 
Recall that in Section 4.4.2, we proposed a strategy for removing test cases to minimize functional test suites and ad hoc test suites which often turn out to contain redundancy. Following the heuristic described in Section 4.4.2, test cases are randomly removed per leaf (rule) unless the percentage of code coverage (we use statement coverage) drops. Obviously statement coverage was not a sufficient critieria for ensuring the reduced test suite kills the same number of mutants as would the initial test suite. One improvement to this strategy would be to combine Statement Coverage with Condition Coverage, which reports the true or false outcome of each boolean sub-expression, since most of the mutants that are not killed involve boundary values in the conditional statements. As discussed in Section 5.7.2, the nine mutants that are not killed by the expert reduced test suite are AOIS102, ROR1, ROR8, ROR11, ROR18, ROR21, ROR28, ROR31, and AOIS31 (see descriptions in Table 13, Appendix D). If condition coverage is used, during the reduction process only two (AOIS102, AOIS31) would have not been killed which is an improvement to our initial strategy. We currently further investigate other strategies to improve this process.

\subsubsection{Evolution Context}

We now discuss the context where we use a student TS with the student CP (Evolution Context). As discussed in Section 4.4.1 and the previous section, two solutions are discussed for adding test cases: The first one is to follow the CP method and build all the feasible (according to properties and selectors) combinations of choices and select the ones that are missing in the abstract test suite, and the second is to identify which combinations of choices may be relevant to determine the output 
class and could be missing from the test suite by using the decision tree. None of the two solutions is inherently better than the other in this context. The reason is that the student CPs' do not have the necessary categories and choices which can be used to add test cases that will kill mutants that would have been killed otherwise. But the argument for using the first solution is that it will result in a larger test suite, meaning that the resultant test suite will have a higher possibility of killing more mutants. An alternative to these two techniques will be investigated in our future work.

When removing test cases, the solution discussed for the OSS context applies to the evolution context as well. For instance, the four mutants (ROR5, ROR13, ROR14, and ROR36) that are not killed by student A's reduced test suite would have been killed if we use condition and statement coverage together.

\subsection{Discussions}

The previous sections illustrated the MELBA iterative process in two different application contexts: (1) when Category-Partition specifications are used to generate an initial, possibly incomplete test suite, (2) when no Category-Partition specification exists and must be reverse-engineered independently from the test suites, based on an intuitive functional understanding of the system under test. In both situations, we showed that using MELBA we were able to identify instances of problems in the decision trees and use this information to improve both test suites and CategoryPartition specifications. The iterative process stopped when no problem could be identified in the trees, at which point the test suites and Category-Partition specifications were reaching a quality level that would likely have been achieved by 
an expert and that was in any case equivalent to the best Category-Partition specifications we could derive.

From the case study, we can also conclude that our taxonomies of decision tree problems and their possible root causes (Sections 4.2 and 4.3) are complete with respect to the PackHexChar program. With the case study performed not all problemcause pairs were covered. For instance, we did not have a scenario where a misclassication led to too many test cases for a rule. We also did not have a scenario where a category is not used because of ill-defined choices.

The resulting test suites for students obtained in section 5.5 and section 5.6 are smaller than the expert TS. There are two reasons why this is the case: the first is that in the heuristic described in section 4.4.1, test cases are added only when needed not based on the Category-Partition, the second reason is that expert $\mathrm{CP}$ has categories and choices that do not impact the output but results in a larger test suite.

Furthermore, we have seen that based on our students' test suites, who can be considered competent testers in terms of training, we could achieve a final CateogryPartition specification and test suite in two to three improvement steps.

Recall the two solutions discussed in Section 4.4.1 for adding test cases and the solution for removing test cases in Section 4.4.2 have defencies as discussed in Section 5.8. The main reason why we were unable to kill all mutants at the end of the improvement process is because our improvement strategy added test cases based on the information provided by the tree and not the CP. The problem with the reduction strategy is that when removing test cases we only consider Statement Coverage, 
which does not seem to be sufficient. One improvement to this strategy would be to consider other code coverage condition such as Condition Coverage.

Of the two application contexts discussed, the evolution context (Section 5.5) requires upfront investment in creating a CP. However, the improvement process is the same for both OSS and Evolution context. The reduction process requires a lot of time since everytime test cases are removed we have to check the code coverage information and keep doing so until we reach a test suite with minimum number of test cases but still have the same code coverage as the improved test suite. 


\section{CHAPTER 6 CASE STUDY TWO: SPACE PROGRAM}

In this section, we first describe the second system used for the case study (Section 6.1). We then present the design of the case study (Section 6.2) and describe the results of applying C4.5 decision trees to drive the improvement of the test specification and test suite (Sections 6.3 and 6.4). Lastly, we discuss the results in section 6.6.

\subsection{The Space Program}

The Space program [35] was originally developed by the European Space Agency, and was first used in a software engineering study by Pasquini et al. [35]. It has subsequently been used in other experiments. Space allows users to describe the configuration of an array of antennas using a specific array definition language (ADL). It reads a text file containing ADL statements, checks its conformance to the ADL grammar as well as specific consistency rules, and performs other computations. It is a 5905-NBNC ${ }^{10}$-LOC C program. (See [36] for further details.) Vokolos and Frankl [37] used the program for a study in which they compared the effectiveness of regression test selection strategies. For that study, they generated 10,000 test cases using a randomized input generation tool. Rothermel et al. [38] later added enough test cases to ensure that each executable decision was covered by at least 30 test cases in each direction; this procedure added 3,585 test cases to the pool.

\footnotetext{
${ }^{10} \mathrm{NBNC}$ : №n-blank line, non-comments statements.
} 
Note that this suggests that the test suite contains redundant test cases. The resulting test pool covers $90,85,85$, and 80 percent of all blocks, decisions, c-uses, and p-uses present in the program, respectively. The total number of blocks, decisions, c-uses, and p-uses are $2995,1191,3733$, and 1707 , respectively.

During the course of their research, Rothermel et al. [38] identified and eliminated five more faults, bringing the total number of versions of the program to 38 ; however, they found that three of the original versions did not exhibit faulty behavior, reducing the number of non-equivalent versions to 35 . We obtained the program, and test pool from the Galileo Research Group Subject Infrastructure Repository at the University of Nebraska - Lincoln. In this case study, we used the Space program [35], and test cases generated by the authors of $[35,38,39]$ directly.

In our method, we use the category partition technique to characterize test cases instead of using this technique to devise test cases. Therefore, it is not necessary to identify constraints on choices as suggested by the category-partition technique [6]. The CP specification available from [23] contains 207 parameters, 83 categories, and 582 choices (Appendix E.1). Since the Space program is a language processing program, it is not surprising that such a large number of categories and choices are defined.

\subsection{Design of the Experiment}

Recall from Figure 4 that the MELBA method we propose can be applied in two broad contexts: The reuse, validation, and integration of open source software and software evolution. The Space program lies in the evolution context. We can however 
further distinguish two situations (in the evolution context) that require we perform two slightly different types of experiments (Sections 6.3 and 6.4), because of the specifics of the test suite of the Space program. In the first experiment (Sections 6.3) we use the CP specification with the original test suite $(13,585$ test cases) to generate the decision tree. In the second experiment (Section 6.4) we use a much smaller test suite (56 test cases) to go through the improvement process. The 56 test cases have been obtained from the original test suite by randomly removing test cases from the original test suite until the statement coverage of the test suite drops. The main reason is that, as suggested by the way the original test suite has been built, the test suite contains a lot of redundant test cases. So we expect to discover many rules with a lot of instances during the first experiment, and we therefore want to exercise the Melba procedure on a more realistic test suite.

Since the output equivalence classes have not been defined for the space program in [23], we have to define them. The experiments are carried out in Sections 6.3 and 6.4 using the following output equivalent classes, which are all the possible (error) messages the Space program returns (please refer to Space program for detailed descriptions of these errors):

i. INV_GEOMETRY_DEF: ERROR 34: Incorrect element GEOMETRY definition.

ii. INV_PHASE_EXC_DEF: ERROR 80: Incorrect PHASE excitation definition.

iii. INV_PORTS_DEF: ERROR 49: Incorrect element PORTS definition

iv. INV_AMP_EXC_DEF: ERROR 79: Incorrect AMPLITUDE excitation definition. 
v. INV_ADD/REMOVE_DEF: ERROR 64: Incorrect ADD or REMOVE statement.

vi. INV_GRID_DEF: ERROR 67: Missing ELEMENT definition after GRID definition.

vii. INV_POLARIZATION_DEF: ERROR 31: Incorrect element LINEAR polarization definition.

viii. NULL_POINTER

ix. SEC_ORD_NOT_ALLOWED_ON_IRREG_GROUPS: ERROR B5: SECOND ORDER law not allowed on irregular groups.

x. ADD_HEX_NOT_ALLOWED_ON_RECT_GEOM: ERROR B8: Error in group geometry

xi. RECT_NOT_ALLOWED_ON_HEX: ERROR B3: RECTANGULAR element not allowed on HEXAGGONAL grid in GROUP

xii. GRID_ANG_OUTOF_NON_AMBIG_RANGE: ERROR B4: GRID angle out of non ambiguity range

xiii. SEQ_ROT_NOT_ALLOWED_ON_IRREG_GROUPS: ERROR B11: SEQUENTIAL ROTATION not allowed on irregular shaped groups.

xiv. SEQ_ROT_NOT_ALLOWED_ON_MULTIPORT_ELEM: ERROR B10: SEQUENTIAL ROTATION not allowed to multiport elements.

xv. FILE_ERROR: File related errors occur when the file does not exit, the file is empty or an incorrect number of parameters is passed

xvi. VALID_INPUT: The test case input is valid and the space program produces an ouput.

We then try to identify occurrences of problems using the heuristics described in Figure 4. We go through the iterative improvement process illustrated in Figure 4 Both the test suite and the $\mathrm{CP}$ specification were iteratively improved using the patterns we specified in Section 4.3 for analyzing decision trees and their potential relationships to problems in the test suite or CP specification. Since we also require 
code coverage information when removing test cases, Coverage Validator ${ }^{11}$, for measuring and reporting $\mathrm{C}$ code coverage, is used for our purpose. Coverage Validator supports statement coverage. The results of applying $\mathrm{C} 4.5$ are reported in Section 6.3 and 6.4 for the two experiments, respectively.

\subsection{Experiment one}

In this experiment we use the complete Space test suite $(13,585$ test cases) with the original Space CP and our 16 output equivalence classes (Section 6.2). We generate the corresponding abstract test suite, and after running $\mathrm{C} 4.5$, we obtain the decision tree in Figure 35: this is only an excerpt of the decision tree, which contains a total of 6,892 rules; the confustion matrix is also very large and is not shown here. There are a number of issues with the tree. First, there are 125 misclassifications, which is relatively small considering that we have 13,585 test cases: $0.9 \%$ of them are misclassified. This suggests that our CP specification, including output equivalence classes is relatively good at specifying Space's functionalities. Second the tree is quite large and therefore difficult to analyze. This suggests that on such a large CP test suite and $\mathrm{CP}$ specification, tool support for Melba is warranted. There is redundancy in the test suite, as discussed earlier, and therefore, as expected, many rules cover more than one test case. The minimum and maximum numbers of test cases in rules are 0 and 339, respectively. Figure 34 shows the distribution of numbers of rules with specific numbers of test cases: more than $4000(\sim 60 \%)$ rules

\footnotetext{
${ }^{11} \mathrm{http}: / / \mathrm{www}$. softwareverify.com/cpp/coverage/index.htm
} 
have one test case, but a large number of rules have more than 10 test cases; 309 rules have more than 30 test cases (recall that each edge and node in the source code control flow graph is covered by at least 30 test cases, but apparently these white box test cases do not correspond to unique combinations of input/output combinations from our $\mathrm{CP}$ specification); redundancy may also ggestsu misclassification (Section 4.3.6).

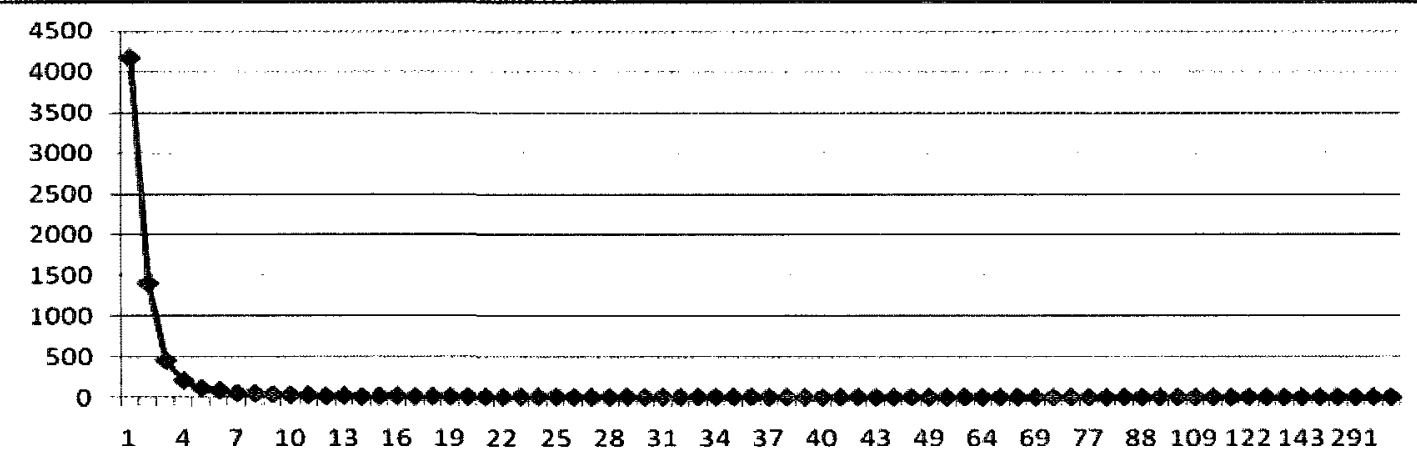

Figure 34 Normal distribution of test cases per rule

A careful analysis of the (huge) tree shows that the tree suggets some combinations of choices are not exercised by the complete test suite. We also make such an observation during the second experiment that we further discuss in the following section. On the one hand this is surprising considering the size of the test suite, and the way (redundancy) it was built. On the other hand, test cases where added to the test suite to increase the amount of code coverage (recall Section 6.1), but coverage of statements, edges, and data flow does not necessarily correspond to combinations of choices which is a functional aspect rather than structural one: we believe that combinations of choices better correspond to paths in the code than simple structural elements as the ones mentioned above. 
In the first scenario we do not report on the complete application of the Melba process since we quickly (first decision tree) reach the limits of the manual application of the process. This also justifies our second experiment, where we apply Melba on a reduced test suite, and the construction of tool support, which is left to future work.

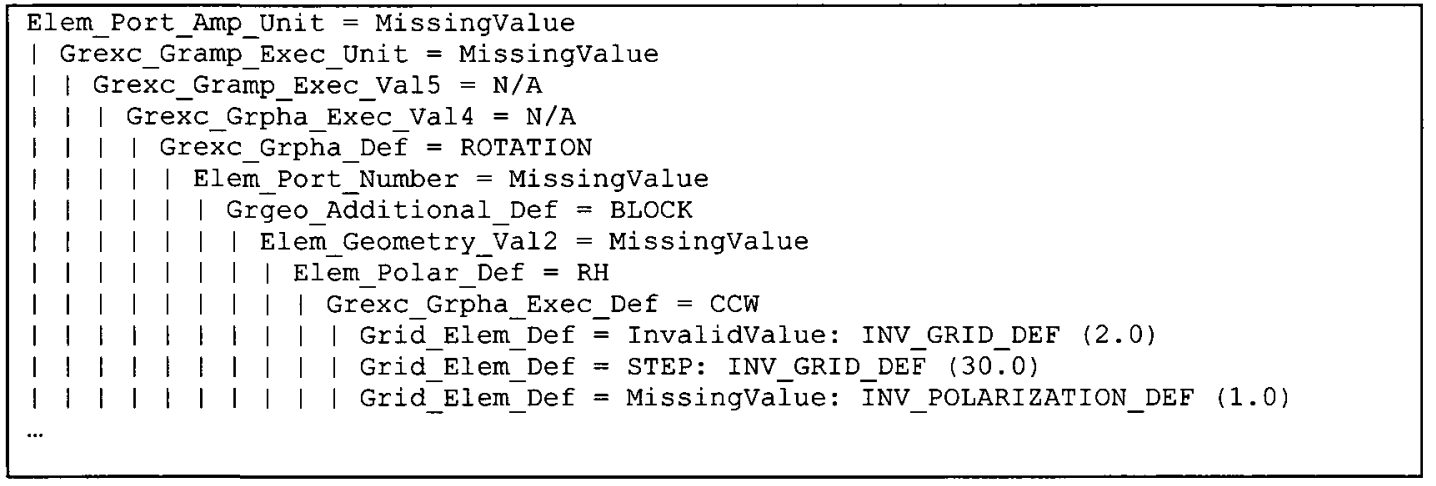

Figure 35 DT with complete TS and Original CP - 16 Equivalence classes

This experiment was also carried out in two different other scenarios where we used two different sets of (coarser grained) output equivalence classes. In one scenario we used only three output equivalence classes: VAL_INPUT, INV_INPUT (representing 14 of the classes of Section 6.2) and FILE_ERROR. In the other scenario, we used only four output equivalence classes: VAL_INPUT, INV_SYNTAX (representing seven, i.e., i-vii, of the classes of Section 6.2), INV_SEMANTICS (representing seven, i.e, viii-xiv of the classes of Section 6.2) and FILE_ERROR. In each scenario, we generated the corresponding abstract test suite, and after running $\mathrm{C} 4.5$, we obtained a decision tree with a very large number of rules (3100 and 3292, respectively), with larger numbers of instances per rule (the minimum and maximum numbers of test cases in rules are 0 and 817 , and 0 and 814 , respectively), which also showed misclassifications (73 and 74 respectively, i.e., $0.5 \%$ of the complete test 
suite in each case). Since these scenarios did not change our previous observations, we do not report on them. In addition, although the number of rules and misclassified instances in trees generated with those coarser grained output equivalence classes is almost half of that of the tree generated with 16 equivalence classes, we believe that the benefit of having a refined analysis with 16 classes provides in depth understanding of the system and better exposure to paths that are not excerscised in the test suite. This will have to be confirmed by additional studies.

\subsection{Experiment two}

In this experiment we use a reduced test suite (56 test cases) with the original Space $\mathrm{CP}$ and our 16 output equivalence classes (Section 6.2). We generated the corresponding abstract test suite and followed the Melba process.

\subsubsection{First Iteration}

After running C4.5 we obtain the decision tree (and confusion matrix) in Figure 36 with 55 rules. The tree in Figure 36 explicitely shows that 23 combinations of choices are missing (Case 3 ), as they correspond to rules with no instances (identified by $(*)$ ).

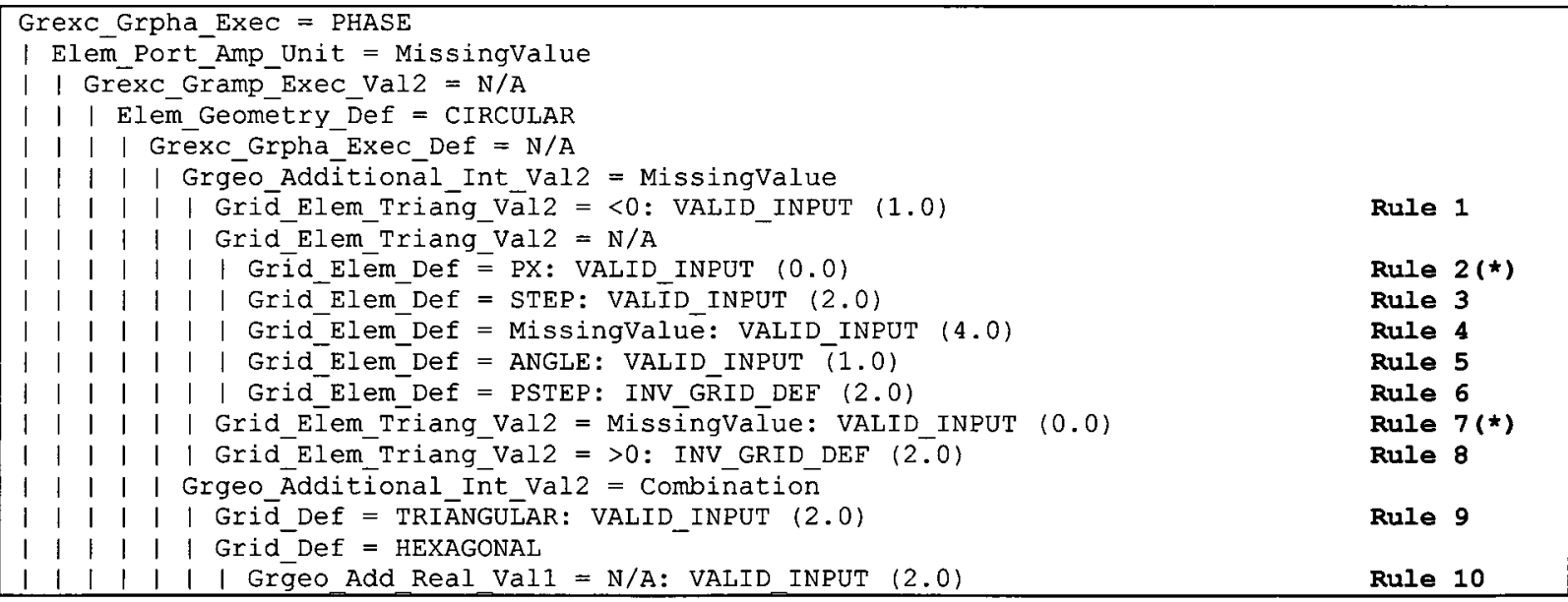




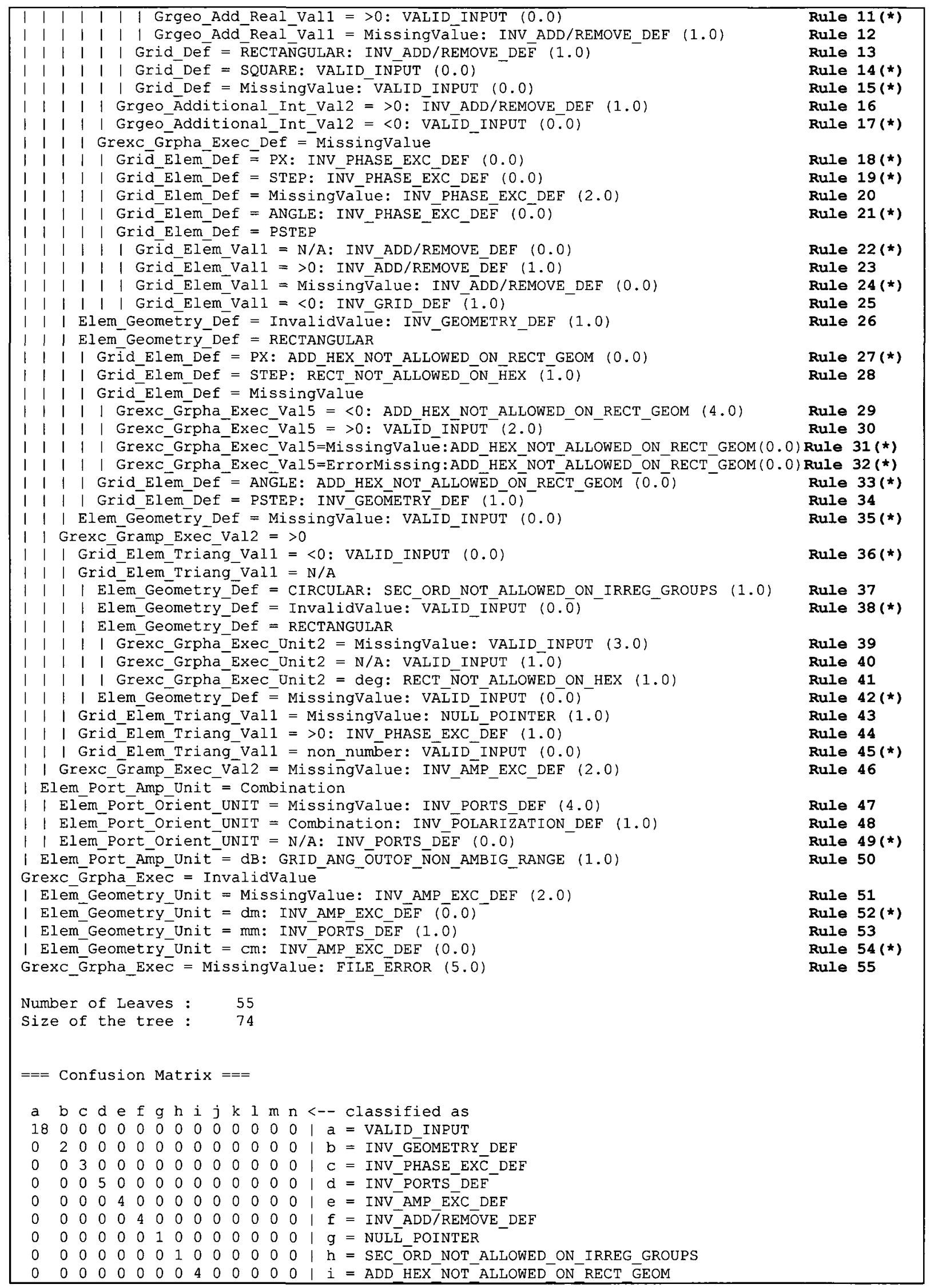




\begin{tabular}{|llllllllllllllll|}
\hline 0 & 0 & 0 & 0 & 0 & 0 & 0 & 0 & 0 & 2 & 0 & 0 & 0 & 0 & $j=$ RECT_NOT_ALLOWED_ON_HEX \\
0 & 0 & 0 & 0 & 0 & 0 & 0 & 0 & 0 & 0 & 1 & 0 & 0 & 0 & $\mathrm{k}=$ INV_POLARIZATION_DEF \\
0 & 0 & 0 & 0 & 0 & 0 & 0 & 0 & 0 & 0 & 0 & 5 & 0 & 0 & $\mathrm{i}=$ INV_GRID_DEF \\
0 & 0 & 0 & 0 & 0 & 0 & 0 & 0 & 0 & 0 & 0 & 0 & 1 & 0 & $\mathrm{~m}=$ GRID_ANG_OUTOF_NON_AMBIG_RANGE \\
0 & 0 & 0 & 0 & 0 & 0 & 0 & 0 & 0 & 0 & 0 & 0 & 0 & 5 & $\mathrm{n}=$ FILE_ERROR \\
\hline
\end{tabular}

Figure 36 DT with reduced TS and Original CP - 16 Equivalence classes

Following the heuristic described in Section 4.4.1 for adding test cases results in the identification of approximately $8,239,381$ missing combinations of choices. We consider this number to be too large and probably an indication that our heuristic for improving the test suite is probably not very adequate for large CP specification and test suite like Space's. Therefore, we lean towards a new heuristic based on the CP specification and decision tree, instead of a heuristic solely based on the decision tree. This new heuristic relies on the notion of base-choice (or nominal choice) which is a notion introduced in the original Category-Partition technique. The heuristic assumes that a base choice, or nominal choice, be defined for each category in the $\mathrm{CP}$ specification. (Base choices for the Space CP specification are available in Appendix E.) Category-Partition then defines a number of criteria for selecting combinations of choices to exercise. One of them, the All Combinations criterion, is to try all the feasible combinations of choices: one value for every choice of every category must be combined with one value of every choice of every other category (as long as the combination satisfies constraints). Another criterion, namely Base Choice, requires that a first base test be formed by using all the base choices for categories. Subsequent tests are chosen by holding all but one base choice constant (i.e., we select a non-base choice for one category) and forming choice combinations by covering all non-base choices of the selected category. This is repeated for each 
category. Instead of All Combinations, which leads to a too large number of test cases, we decided to use Base choice coverage.

Keep in mind, though, that we still want to rely on the information provided in the decision tree. Therefore, we only use categories and choices selected by the tree. Our heuristic is therefore defined as follows. We first identify combinations of choices that the tree suggests are missing (this is similar to the first heuristic discussed in Section 4.4.1). We then only add a test case for a combination if the combination has at most one non base choice.

Following the heuristic described above, we add a test case for a missing combination of choices only if the combination of choices it triggers (as indicated by the tree) contains at most one non base choice - there are 12 such instances. Of the 12 new choice combinations, only two abstract test cases matching the choice combinations were found in the complete Space test suite. Test cases for the remaining ten choice combinations identified in Figure 37 were added and the Abstract Test Cases generated.

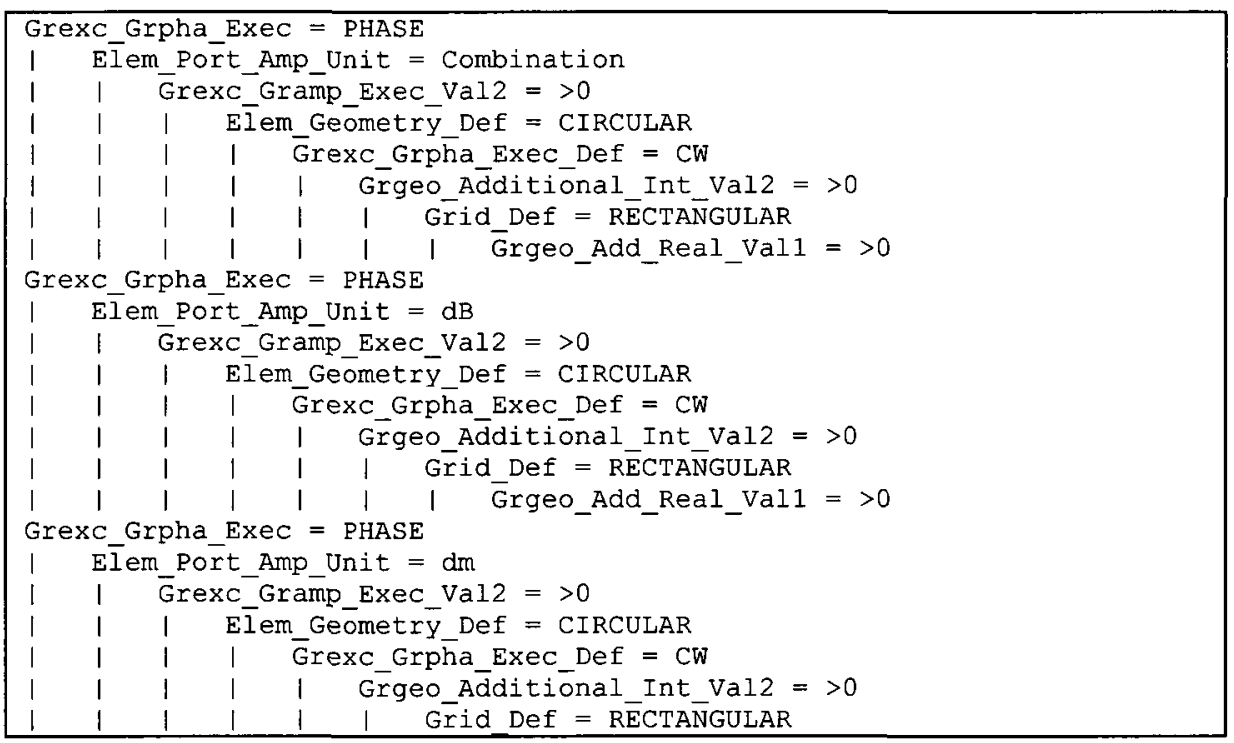




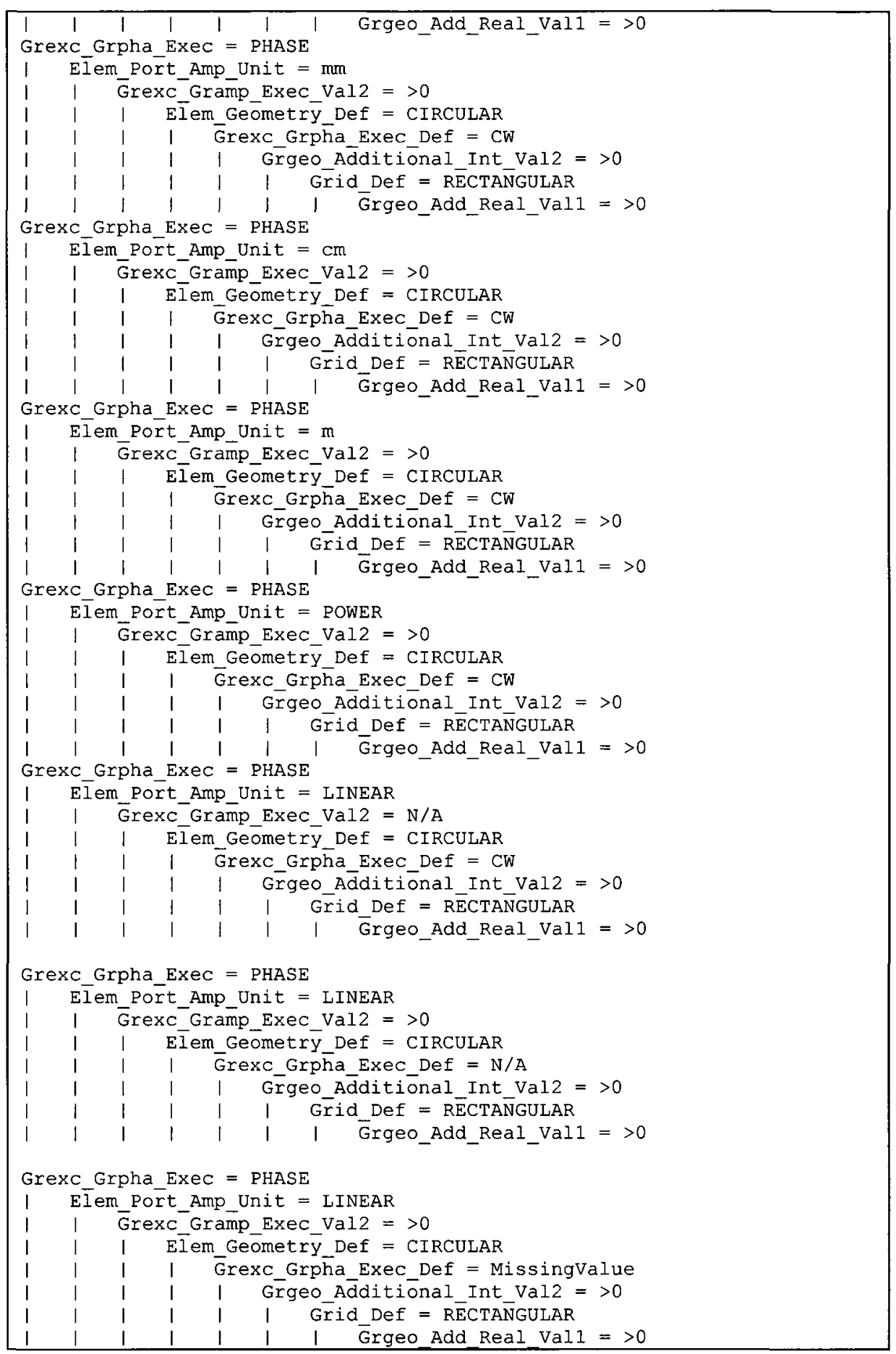

Figure 37 Missing combinations of choices for TC's are added

To add the test cases for these 12 combinations of choices we proceeded as follows.

For the two missing combinations that are exercised by some test cases in the 
complete test set, instead of creating test cases from scratch, we reused the complete test set as follows: (i) We identified the test cases in the complete test set that exercise each missing combination of choices; (ii) We randomly selected one such test case for each missing combination. For the ten missing combinations (Figure 37) that are not exercised by the complete test set, we specifically created test cases from scratch. The results are shown in the $2^{\text {nd }}$ iteration.

It is worth recalling that test cases in the test suite are mostly white-box test cases (e.g., to cover several times specific control and data flow elements) and therefore do not necessarily exercise specific functional aspects of the system, as specified by choice combinations. This first iteration of the Melba process does illustrate the fact that the process can lead to improvements to an industrially developed test suite: we found new interesting functional test cases for Space.

\subsubsection{Second Iteration}

Once the $56+12=68$ abstract test cases are (automatically) re-created from the improved TS, the execution of C4.5 produces the decision tree of Figure 38 made of 80 rules. The tree shows that some choice combinations are missing (Case 3), 39 of them being explicitly identified in rules with no instance (identified with a $(*)$ ), with some combinations feasible and some unfeasible.

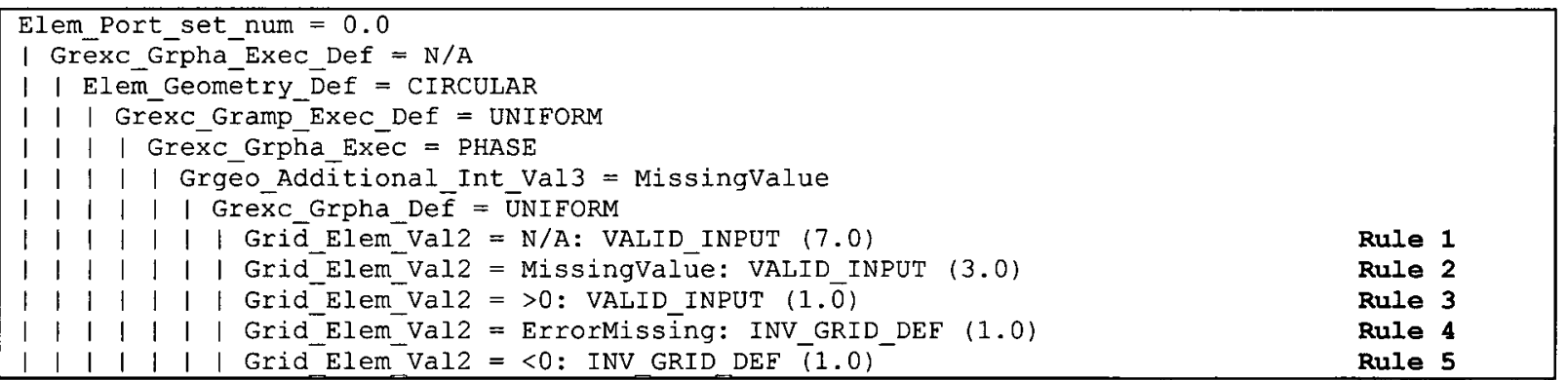




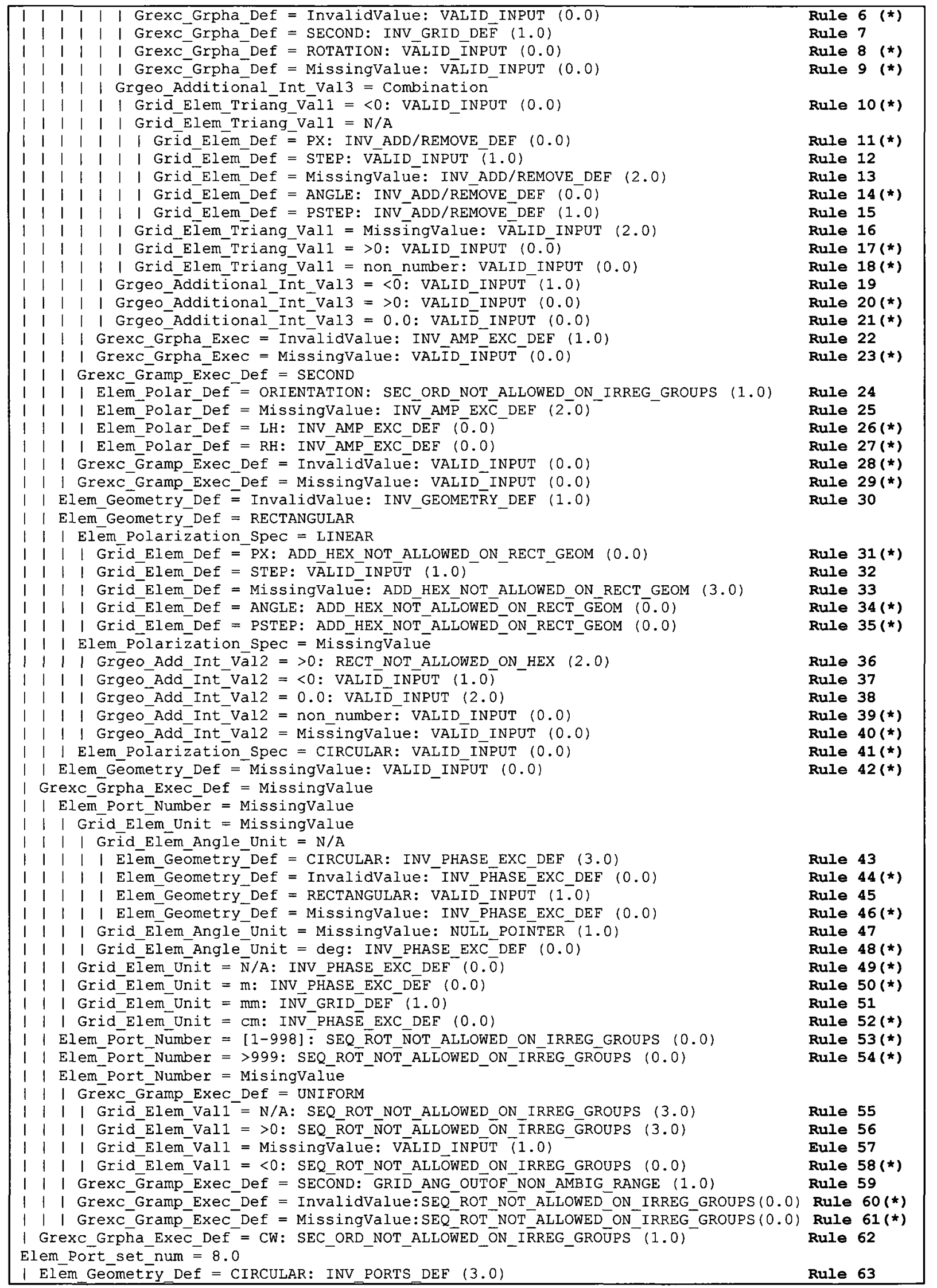




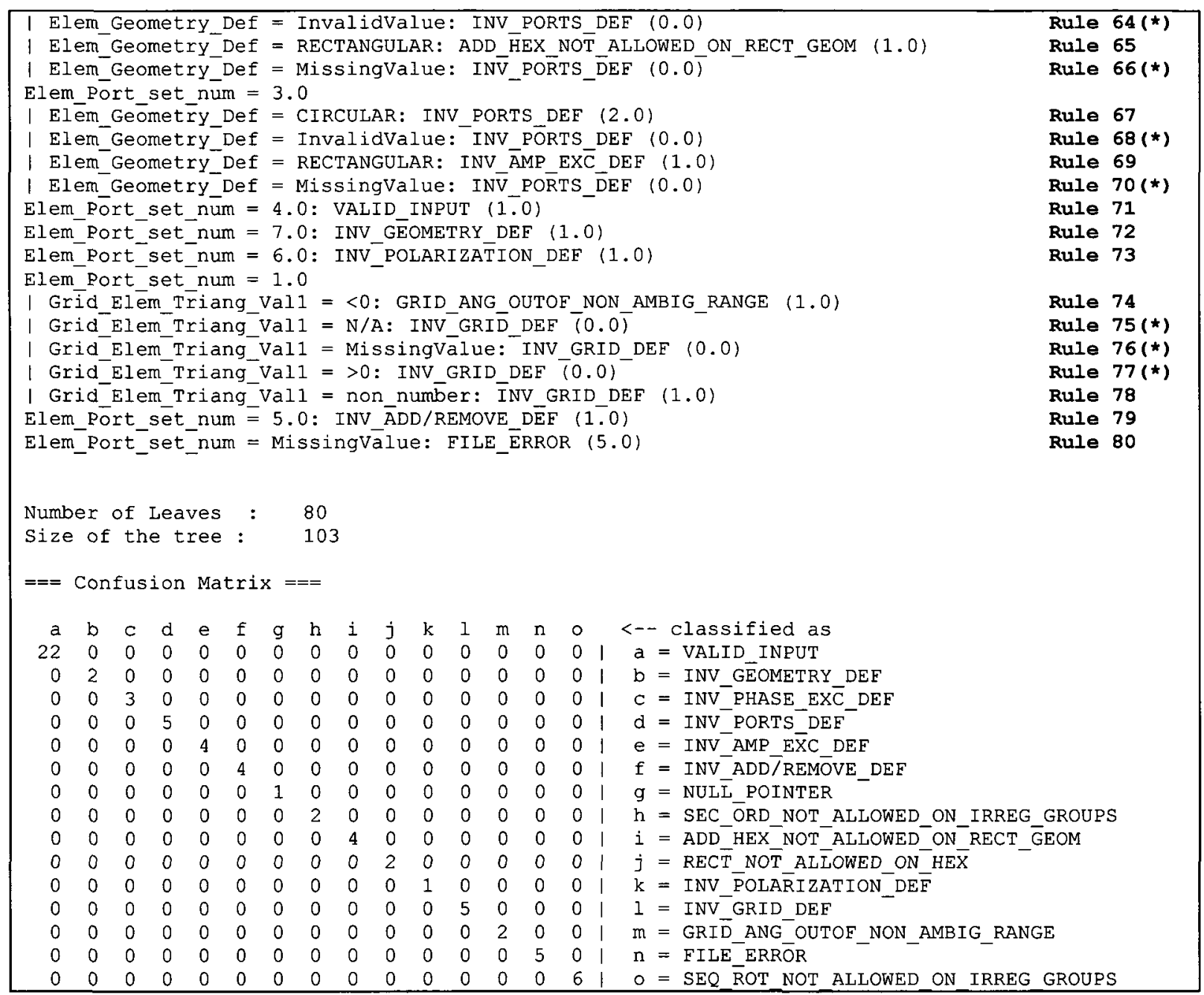

Figure 38 Space Decision Tree - Second Iteration

In order to reach the final iteration where no occurrence of the problems discussed in Section 4.2 can be found in the resultant decistion tree, we believe that it would require quite a large number of iterations, which appears to be unfeasible without tool support in the context of this thesis. Therefore, we stopped at that stage of the application of the Melba process. 


\subsection{Melba Process Automation - PackHexChar and Space}

As indicated in Figure 4, ativity 1 is fully automated. In our case study, we automated this activity through custom tools written in Java using Eclipse IDE. For each case study, a tool takes as input the CP specification and the raw test data; the tool then generates the abstract test cases by mapping each value in the test case to the appropriate choice(s) in the $\mathrm{CP}$ specification. The output file is a comma delimited (.csv) file where the last column indicates the output equivalence class, this file is refered to as the abstract test suite (ATS).

The file (ATS) generated in Activity 1 is in the format acceptable by the WEKA tool, which implements C4.5 to generate the DT (In Activity 2). For the packhexchar case study, it took less than a second for WEKA to generate a decision tree whereas it took eight seconds to generate a tree based on 13,585 abstract test cases for Space. (The execution times are reported on a PC with windows XP, AMD Athlon 4200+ processor, and a 2 GB RAM)

Activities 3, 4 and 5 are not automated at this point, as they rely on the know-how and expertise of the tester. However, we provide guidance to help identify which categories/choices need to be refined and which abstract test cases need to be defined. Test suite amendment (Activity 4) requires an effort that is anyway incurred if one is improving a test suite. Note however that this effort (e.g., building the test harness that include drivers and oracles) will only involve the newly created test cases: the tester will be able to reuse drivers and oracles for existing test cases. 


\subsection{Discussions}

The experiments on the Space program are carried out on a much larger problem than in CHAPTER 5: much larger test suite (though it contains redundancy) and much larger CP specification. We conducted in fact two different experiments in the Space program.

In the first experiment, we used the complete test suite (with 13,585 test cases). After the first iteration of the Melba process, the decision tree suggested that there are category-choice combinations missing in the test suite, potentially indicating that some paths are not excercised by the initial (complete) test suite.

In the second experiment we reduce the complete test suite by randomly removing test cases until further test suite reduction causes statement coverage to drop: we obtain a smaller test suite, equivalent to the initial, large one in terms of statement coverage. We then justify test case addition using the CP method and the BaseChoice (Nominal-Choice) criterion. During the first iteration of MELBA we were able to identify instances of problems in the decision trees and use this information to improve the test suite. In particular, the decision tree pointed to missing combinations of choices, some of which are not even exercised by the complete test suite (with 13,585 test cases). With retrospect, this is not entirely surprising since the complete test suite mostly contains white-box test cases (e.g., to cover several times specific control and data flow elements) and therefore does not necessarily exercise specific functional aspects of the system, as specified by choice combinations. 
In both experiments, due to lack of tool support for the Melba process, we limited our investigation: the problem is too large to be handled manually. We stopped early in the Melba process and were unable to show the final iteration where no problem could be identified in the generated trees.

Not all problem-cause pairs are illustrated in this case study. Better tool support will help reduce manual intervention and will enable applying our technique to applications with larger CP specifications. Tool support for Melba is the purpose of future work. 


\section{CHAPTER 7 CONSLUSIONS AND FUTURE WORK}

This thesis proposed the MELBA automated iterative method, based on machine learning, to help software engineers analyze the weaknesses of test specifications and test suites so as to be able to iteratively improve them.

The MELBA method takes as inputs the test suite and specifications to be reengineered, the latter following the Category-Partition (CP) strategy. Based on the Category-Partitions specification, test cases are transformed into abstract test cases which are tuples of pairs (category, choice) associated with an output equivalence class (instead of raw inputs/outputs). A machine learning algorithm (in our case, C4.5) is used to learn rules that relate pairs (category, choice), modeling input properties, to output equivalence classes. These rules are in turn analyzed to determine potential improvements of the test suite (e.g., redundant test cases, need for additional test cases) as well as improvements of the Category-Partition specification (e.g., need to add a category or choice).

We have illustrated the main aspects of the MELBA method on a running example (the Triangle program), and evaluated its effectiveness on test suites and CategoryPartition specifications created by fully trained $4^{\text {th }}$ year students on a small size but logically complex program. The study showed that the iterative process can indeed improve both the test suite and the Category-Partition specification to a level that is equivalent to what an expert would likely produce within two to three improvement cycles. An evaluation of the (improved) test suite effectiveness at finding faults (i.e., 
killing mutants) showed that the improved test suites kill more mutants than the original test suites. When removing redundant test cases though, the reduced test suite may not always detect the same faults as the improved test suite (though the drop is small), thus suggesting a trade-off between test cost and effectiveness. But as discussed in section 5.9, in our future work, we look into improving the test suite reduction heuristic by looking at different code coverage techniques (we used statement coverage as it is simple to apply, though it is known to not be sufficient-it has to be completed with edge coverage and/or some data flow coverage criterion).

We performed a second evaluation of the MELBA method on a more complex, industrial program, the Space program. The Space program originally had 13,585 test cases. In the first experiment, using 16 output equivalence classes defined in section 6.2, we generated a decision tree with 6892 rules based on the expert $\mathrm{CP}$ and complete TS. This appears to be too much if handled manually, as it was the case in this thesis. Tool support for Melba is warranted. In order to show the effectiveness of our technique, we then reduced the test suite to 56 test cases by randomly removing test cases while still keeping the original code coverage (i.e. statement coverage). Due to the lack of tool support, we only show one iteration of the improvement process in this second experiment. The decision tree suggests improvements to the test suite: there are missing combinations of choices that require the addition of test cases. To add the missing test cases, we selected adequate test cases (i.e., that would exercise the missing combinations) from the complete test suite. One interesting result is that some of the missing combinations of choices, as indicated by the decision tree appear to be missing in the complete test suite (i.e., none of the 13,585 test cases exercise 
them). This is not entirely surprising since the complete test suite mostly contains white-box test cases (e.g., to cover several times specific control and data flow elements) and therefore does not necessarily exercise specific functional aspects of the system, as specified by choice combinations. This shows that even on this larger problem, with a very large test suite that has been built with care by other researchers, the Melba process is able to suggest improvements to the test suite.

Future work will include investigating other black-box specifications than CategoryPartition, such as Boolean expressions and statecharts, additional evaluations of the iterative process on programs of varying sizes and complexities to illustrate the problem-causes pairs (Figure 7) that are not illustrated in our case studies, as well as user-friendly automated tool support. (Recall that most of the activities of the MELBA method are automated.) Other strategies to remove redundancy in a test suite will also be investigated, thereby trying to not lose in terms of effectiveness. As noticed during the case studies when adding test cases, not all mutants were killed. Since our test cases addition is based on the deicision tree, therefore, not all categories and choices are considered when adding test cases. Therefore, a new approach is to be investigated where both the decision tree and the CP specification are used when adding test cases, instead of solely using the decision tree. 


\section{REFERENCES}

[1] H. Muccini, "Software Architucture for Testing, Coordination and Views Model Checking," in Computer Science: University of L'Aquila, 2002.

[2] P. C. Jorgensen, Software Testing: A Craftsman's Approach 2nd ed.: CRC Press, 1995.

[3] M. Michlmayr, F. Hunt, and D. Probert, "Quality practices and problems in free software projects," in First International Conference on Open Source Systems, 2005, pp. 24-28.

[4] L. Zhao and S. Elbaum, "Quality assurance under the open source development model," Journal of Systems and Software, vol. 66, pp. 65-75, 2003.

[5] S. Demeyer, S. Ducasse, and O. Nierstrasz, Object-Oriented Reengineering Patterns: Morgan Kaufmann, 2003.

[6] T. J. Ostrand and M. J. Balcer, "The Category-Partition Method for Specifying and Generating Functional Test," Communications of the ACM, vol. 31, pp. 676686, June 1988.

[7] J. R. Quinlan, C4.5: Programs for Machine Learning: Morgan Kaufmann, 1993.

[8] G. Ammons, R. Bodik, and J. R. Larus, "Mining Specifications," in $A C M$ Principles of Programming Languages, 2002, pp. 4-16.

[9] J. F. Bowring, J. M. Rehg, and M. J. Harrold, "Active Learning for Automatic Classification of Software Behavior," in ACM International Symposium on Software Testing and analysis, 2004, pp. 195-205.

[10]M. Harder, J. Mellen, and M. D. Ernst, "Improving Test Suites via Operational Abstraction," in $25^{\text {th }}$ International Conference on Software Engineering, Portland, OR, USA, 2003, pp. 60-71.

[11]M. D. Ernst, J. Cockrell, W. G. Griswold, and D. Notkin, "Dynamically discovering likely program invariants to support program evolution," IEEE Transaction on Software Engineering, vol. 27, pp. 1-25, 2001.

[12]C. Pacheco and M. D. Ernst, "Eclat: Automatic generation and Classification of Test Inputs," in European Conference on Object-Oriented Programming, 2005, pp. 504-527.

[13]C. Csallner and Y. Smaragdakis, "DSD-Crasher: A Hybrid Analyusis Tool for Bug Finding," in ACM International Symposium on Software Testing and Analysis, 2006, pp. 245-254.

[14]S. Elbaum and M. Diep, "Profiling Deployed Software: Assessing Strategies and Testing Opportunities," Transactions on Software Engineering, vol. 31, pp. 312327, 2005. 
[15] M. Haran, A. Karr, M. Last, A. Orso, A. Porter, A. Sanil, and S. Fouche, "Techniques for Classifying Executions of Deployed Software to Support Software Engineering Tasks," Transactions on Software Engineering, vol. 33, pp. $1-18,2007$.

[16]B. Baudry, F. Fleurey, and Y. Le Traon, "Improving Test Suites for Efficient Fault Localization," in Internatilnal Conference on Software Engineering, 2006, pp. 82-91.

[17] J. A. Jones, M. J. Harrold, and J. Stasko, "Visualization of Test Information to Assist Fault Localization," in Proceedings of ICSE'02 (Int. Conference in Software Engineering), Orlando, FL, USA, May 2002.

[18]D. E. Goldberg, Algorithms in Search Optimization and Machine Learning: Addison-Wesley, 1989.

[19]N. Baskiotis, M. Sebag, M.-C. Gaudel, and S. Gouraud, "A Machine Learning Approach for Statistical Software Testing," in Twentieth International Joint Conference on Artificial Intelligence, Hyderabad, India, 2007, pp. 2274-2279.

[20]H. Singh, M. Conrad, and S. Sadeghipour, "Test Case Design Based on Z and the Classification-Tree Method," in Proceedings of $t^{e}$ 1st International Conference on Formal Engineering Methods, 1997, pp. 81-90.

[21]M. Grochtmann and K. Grimm, "Classification Trees for Partition Testing," Software Testing, Verification and Reliability, vol. 3, pp. 63-82, 1993.

[22]P. Francis, D. Leon, M. Minch, and A. Podgurski, "Tree-Based Methods for Classifying Software Failures," in Proc. International Symposium on Software Reliability Engineering, 2004, pp. 451-462.

[23]L. C. Briand, Y. Labiche, and X. Liu, "Using Machine Learning to Support Debugging with Tarantula," Ottawa, Technical Report February 2007.

[24]I. H. Witten and E. Frank, Data Mining: Practical Machine Learning Tools and Techniques 2nd ed.: Morgan Kaufman, 2005.

[25] W. W. Cohen and Y. Singer, "Simple, Fast, and Effective Rule Learner," in $A A A I / I A A I, 1999$, pp. 335-342.

[26]R. Kohavi and F. Provost, "Glossary of Terms," Editorial for the Special Issue on Applications of Machine Learning and the Knowledge Discovery Process, vol. 30, pp. 271 - 274, February/March 1998.

[27]P. D. L. Machado, "Testing from Structured Algebraic Specifications: The Oracle Problem," in College of Science and Engineering. School of Informatics: University of Edinburg, July 2000.

[28]B. Marick, The Craft of Software Testing: Prentice Hall, 1995.

[29]Emma, "A free Java code coverage tool," http://emma.sourceforge.net/index.html, 2001-2005. 
[30]R. J. Lipton, R. A. DeMillo, and F. G. Sayward, "Hints on test data selection: help for the practicing programmer.," IEEE Computer, vol. 11, pp. 34-41, April 1978.

[31]S. Kim, J. Clark, and J. McDermid, "Class Mutation: Mutation Testing for Object-Oriented Programs," in OOSS: Object-Oriented Software Systems, Germany, Octobor 2000.

[32] Y.-S. Ma, J. Offutt, and Y. R. Kwon, "MuJava: A Mutation System for Java," International Conference on Software Engineering, pp. 827-830, May 20-28, 2006.

[33]J. H. Andrews, L. C. Briand, and Y. Labiche, "Is Mutation an Appropriate Tool for Testing Experiments?," in Proc. of IEEE International Conference on Software Engineering (ICSE) 2005, St. Louis, Missouri, USA, 2005, pp. 402411.

[34]J. H. Andrews, L. C. Briand, Y. Labiche, and A. S. Namin, "Using Mutation Analysis for Assessing and Comparing Testing Coverage Criteria," IEEE Transactions on Software Engineering, vol. 32 (8), pp. 608-624, August 2006.

[35]A. Pasquini, A. Crespo, and P. Matrelle, "Sensitivity of reliability-growth models to operational profiles errors vs testing accuracy," IEEE Transactions on Reliability, vol. 45, pp. 531-540, 1996.

[36] W. E. Wong, J. R. Horgan, A. P. Mathur, and A. Pasquini, "Test Set Size Minimization and Fault Detection Effectiveness: A Case Study in a Space Application," Software Engineering Research Center (SERC), Technical Report TR-173-P, 1997.

[37]F. I. Vokolos and P. G. Frankl, "Empirical evaluation of the textual differencing regression testing technique," in IEEE International Conference on Software Maintenance, Bethesda, MD, USA, 1998, pp. 44-53.

[38]G. Rothermel, R. H. Untch, C. Chu, and M. J. Harrold, "Prioritizing test cases for regression testing," IEEE Transactions on Software Engineering, vol. 27, pp. 929-948, 2001.

[39]L. C. Briand, Y. Labiche, and Z. Bawar, "Using Machine Learning to Refine Black-box Test Specifications and Test Suites," Software Quality Engineering Laboratory, Carleton University TR SCE-07-05, 2007.

[40]Usama M. Fayyad and Keki B. Irani, "On the Handling of Continuous-Valued Attributes in Decision Tree Generation," Machine Learning, vol 8, pp. 87-102, 1992. 


\section{Appendix A CP Specifications}

\section{A.1 Triangle Program}

\section{A.1.1 Description}

The input values $(a, b, c)$ characterize the length of triangle sides. We use Category-Partition and define properties and relationships between sides. We then perform the mapping between the test cases and Category-Partition in order to generate an abstract test suite. Based on the abstract test suite, the learning algorithm will be able to determine whether the triangle is Equilateral, Isosceles, Irregular, not a triangle, or a null triangle.

\section{A.1.2 CP Specifications}

\section{Parameter a}

Category 1 -values for a

Choice C1: $\quad \mathrm{a}>0$

Choice C2: $\quad a<=0$

Category 2 -a compared to $b$

Choice C3: $\quad a=b$

Choice C4: $\quad a !=b$

Category 3-a compared to $b$ and $c$

Choice C5: $\quad a<=b+c$

Choice C6: $\quad a>b+c$

Parameter b

Category 4-values for $\mathrm{b}$ Choice C7: $\quad b>0$

Choice C8: $\quad b<=0$

Category $5-b$ compared to $\mathrm{c}$

Choice C9: $\quad \mathrm{b}=\mathrm{c}$

Choice C10: $\quad$ b $!=c$

Category $6-b$ compared to a and $c$

Choice C11: $\quad \mathrm{b}<=\mathrm{a}+\mathrm{c}$

Choice C12: $\quad b>a+c$

Parameter c

Category 7-values for $\mathrm{c}$

Choice C13: $\quad c>0$

Choice C14: $\quad c<=0$

Category $8-c$ compared to a

Choice C15: $\quad \mathrm{c}=\mathrm{a}$

Choice C16: $\quad c !=a$

Category $9-c$ compared to $a$ and $b$ 


\section{Choice C17: $\quad \mathrm{c}<=\mathrm{a}+\mathrm{b}$}

Choice C18: $\quad c>a+b$

\section{A.1.3 Triangle Decision Tree}

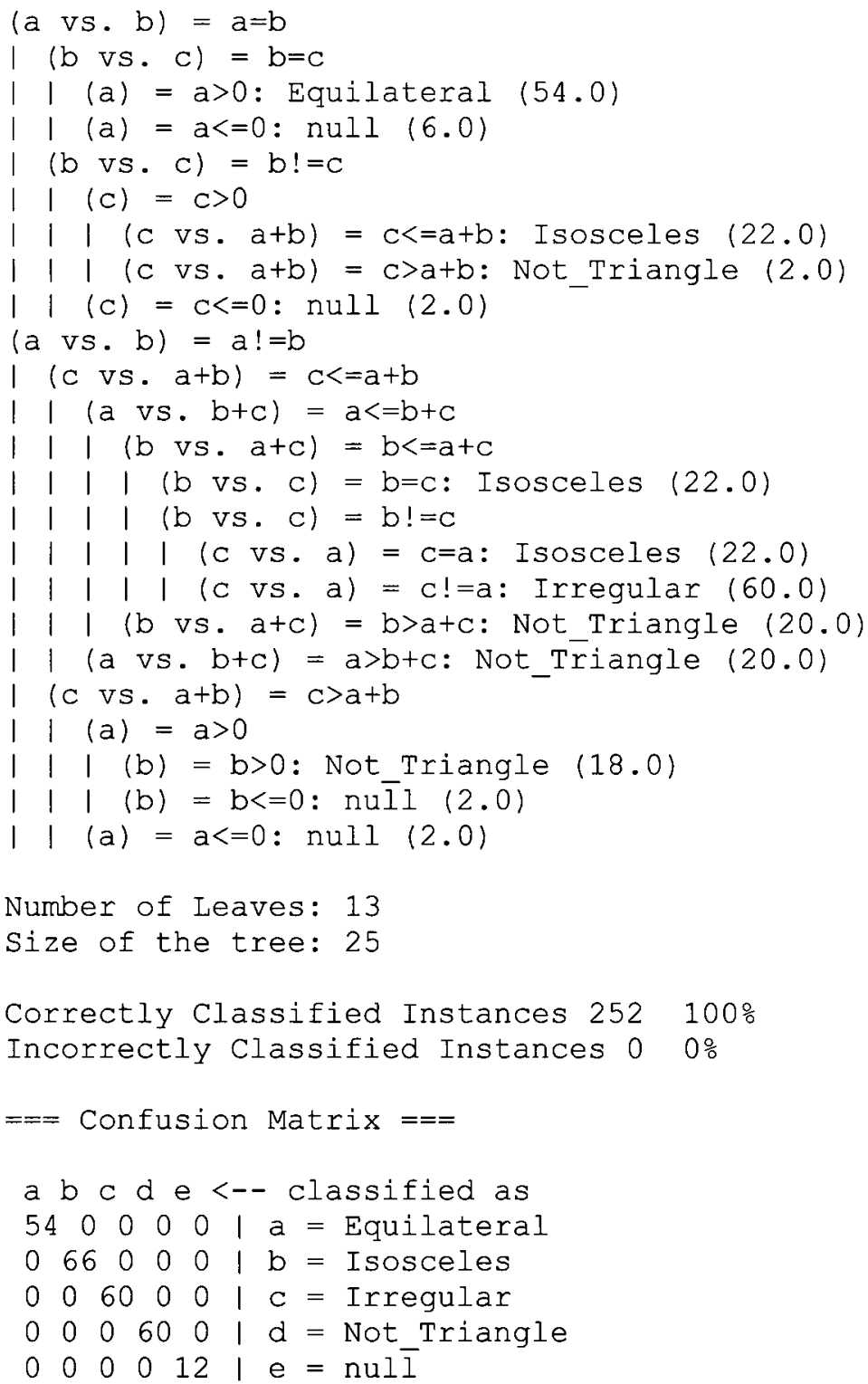




\section{A.1.4 Test Suite for the Triangle program}

\begin{tabular}{|l|l|l|l|}
\hline (a) & (b) & (c) & (Class) \\
\hline 1 & 1 & 1 & Equilateral \\
\hline 1 & 1 & 2 & Isosceles \\
\hline 6 & 6 & 10 & Isosceles \\
\hline 8 & 9 & 9 & Isosceles \\
\hline 1 & 1.5 & 2 & Irregular \\
\hline 3 & 5 & 7 & Irregular \\
\hline 1 & 1 & 2.5 & Not Triangle \\
\hline 0.5 & 1 & 2.5 & Not Triangle \\
\hline-1 & 1 & 1 & null \\
\hline
\end{tabular}

Table 4 Test Cases for the Triangle Program

\begin{tabular}{|c|c|c|c|c|c|c|c|c|c|}
\hline (a) & (b) & (c) & (a vs. b) & (b vs. c) & (c vs. a) & $(\mathbf{a}$ vs. $\mathbf{b}+\mathbf{c})$ & $(b$ vs. $a+c)$ & $(\mathbf{c}$ vs. $\mathbf{a}+\mathbf{b})$ & Class \\
\hline$a>0$ & $b>0$ & $c>0$ & $a=b$ & $b=c$ & $\mathrm{c}=\mathrm{a}$ & $a<=b+c$ & $\mathrm{~b}<=\mathrm{a}+\mathrm{c}$ & $c<=a+b$ & Equilateral \\
\hline$a>0$ & $b>0$ & $c>0$ & $a=b$ & $\mathrm{~b} !=\mathrm{c}$ & $\mathrm{c} !=\mathrm{a}$ & $a<=b+c$ & $\mathrm{~b}<=\mathrm{a}+\mathrm{c}$ & $c<=a+b$ & Isosceles \\
\hline$a>0$ & $b>0$ & $c>0$ & $a=b$ & $\mathrm{~b} !=\mathrm{c}$ & $\mathrm{c} !=\mathrm{a}$ & $a<=b+c$ & $b<=a+c$ & $c<=a+b$ & \\
\hline$a>0$ & $b>0$ & $c>0$ & $a !=b$ & $b=c$ & $c !=a$ & $a<=b+c$ & $\mathrm{~b}<=\mathrm{a}+\mathrm{c}$ & $\mathrm{c}<=\mathrm{a}+\mathrm{b}$ & Isosceles \\
\hline$a>0$ & $b>0$ & $c>0$ & $a !=b$ & $\mathrm{~b} !=\mathrm{c}$ & $\mathrm{c} !=\mathrm{a}$ & $a<=b+c$ & $\mathrm{~b}<=\mathrm{a}+\mathrm{c}$ & $\mathbf{c}<=\mathbf{a}+\mathbf{b}$ & Irres \\
\hline$a>0$ & $b>0$ & $c>0$ & $a !=b$ & $\mathrm{~b} !=\mathrm{c}$ & $\mathrm{c} !=\mathrm{a}$ & $a<=b+c$ & $\mathrm{~b}<=\mathrm{a}+\mathrm{c}$ & $\mathrm{c}<=\mathrm{a}+\mathrm{b}$ & Irregular \\
\hline$a>0$ & $b>0$ & $c>0$ & $a=b$ & $\mathrm{~b} !=\mathrm{c}$ & $\mathrm{c} !=\mathrm{a}$ & $a<=b+c$ & $\mathrm{~b}<=\mathrm{a}+\mathrm{c}$ & $c>a+b$ & Not Triangle \\
\hline$a>0$ & $b>0$ & $c>0$ & $a !=b$ & $\mathrm{~b} !=\mathrm{c}$ & $\mathrm{c} !=\mathrm{a}$ & $a<=b+c$ & $\mathrm{~b}<=\mathrm{a}+\mathrm{c}$ & $c>a+b$ & Not Triangle \\
\hline $\mathbf{a}<=0$ & $b>0$ & $c>0$ & $a !=b$ & $\mathrm{~b}=\mathrm{c}$ & $\mathrm{c} !=\mathrm{a}$ & $a<=b+c$ & $b>a+c$ & $c>a+b$ & null \\
\hline
\end{tabular}

Table 5 Abstract Test Cases using the Test Cases of Table 4 and CP specification

of Section A.1.2 


\section{A.2 PackHexChar: CP Specifications}

\section{Parameter RLEN}

CATEGORY 1-Valid values for rlen
C1: $\quad$ rlen $=0$
[Property rlen0]
C2: $\quad$ rlen $=[1 \ldots$ sLength $] \quad$ [Property rlen 1 ToS]

CATEGORY 2-Invalid values for rlen
C3: $\quad$ rlen $<0$
[Error] [Single] [Property rlenL T0]
C4: $\quad$ rlen $>$ sLength
[Error] [Single]

\section{Parameter ODD_DIGIT}

CATEGORY 3 3-Valid values for odd_digit

C5: odd_digit $=-1$

C6: odd_digit $=[0 \ldots 9]$

C7: odd_digit $=[\mathrm{A} \ldots \mathrm{F}]$

C8: odd_digit $=[\mathrm{a} \ldots \mathrm{f}]$

CATEGORY 4-Invalid values for odd_digit
C9: odd digit $<-1$
C10: odd_digit $>15$
[Error] [Single]
[Error] [Single]

Parameter String $\mathbf{S}$

CATEGORY 5-Length of $S$
C11: $\quad$ sLength $=0$
[Property S0]
C12: $\quad$ sLength $>0$
[Property SGT0]

CATEGORY 6-Type of characters in the first rlen characters of $S$

C13: AllHexadecimal [Property allHD] [if SGT0 AND not rlen0 AND not rlenLT0]

C14: AllNonHexadecimal [Property allNHD] [if SGT0 AND not rlen0 AND not rlenLT0]

C15: MixedChars [Property Mixed] [if SGT0 AND not rlen0 AND not rlenLT0]

CATEGORY 7- Case of chars in the first rlen chars of the string $S$
C16: allNumbers
C17: allLowerCase
[if not allNHD AND not rlen0 AND not rlenLT0]
C18: allUpperCase
[if not allNHD AND not rlen0 AND not rlenLT0]
C19: $\quad$ MixedCase
[if not allNHD AND not rlen0 AND not rlenLT0]
[if not allNHD AND not rlen0 AND not rlenLT0]

CATEGORY 8- The first rlen characters of the string contains boundary Value $[0,9, a, f, A$, F]
C20: Contains [0]
[Single] [if MixedCase]
C21: Contains [9]
[Single] [if MixedCase]
C22: Contains [a]
[Single] [if MixedCase]
C23: Contains [f]
[Single] [if MixedCase]
C24: Contains [A]
[Single] [if MixedCase]
C25:
C26:
Contains [F]
[Single] [if MixedCase]
C27:
ContainsMixed
[Single] [if MixedCase]

CATEGORY 9-Number of hexadecimal characters in the first rlen characters of $S$

C28: Odd

C29: Even

[if not rlenLT0]

[if not rlenLT0] 
C30: Zero

CATEGORY 10-Position of the first non-hexadecimal character in the first rlen characters of $\mathbf{S}$

C31: First [Single] [if SGT0 AND Mixed]

C32: Middle [Single] [if SGT0 AND Mixed]

C33: Last [Single] [if SGT0 AND Mixed]

CATEGORY 11-Consecutive non-hexadecimal characters in the first rlen characters of $S$

C34: Yes

C35: No [if SGT0 AND not rlen0 AND not rlenLT0]

\section{A.2.1 Note on PackHexChar CP Specifications:}

Given the selectors on the choices, some combinations may not feasible; in some cases a whole category will be skipped. For instance when $r l e n<0$ then the following categories $6,7,8$, and 9 are skipped, instead a value of $\mathrm{N} / \mathrm{A}$ is placed in the ATS. Or for instance, if the string $\mathrm{S}$ contains all hexadecimal characters then category 8 does not apply since there are no non-hexadecimal characters and therefore a N/A is placed in the ATS to indicate that a category was skipped because of the selectors on the choices. 


\section{A.2.2 Mapping between Abstract Test Suite and CP}

\begin{tabular}{|c|c|c|}
\hline Categories in the CP & Choices in the CP & $\begin{array}{l}\text { Output } \\
\text { Equivalence } \\
\text { Class }\end{array}$ \\
\hline $\begin{array}{l}\text { Category 1: Contains } \\
\text { choices for all the } \\
\text { valid values of RLEN }\end{array}$ & $\begin{array}{l}\text { C1: RLEN }=0 \\
\text { C2: RLEN is between } 1 \text { and the } \\
\text { length of } S\end{array}$ & \multirow[b]{2}{*}{ RLEN } \\
\hline $\begin{array}{l}\text { Category 2: Contains } \\
\text { all invalid values of } \\
\text { RLEN }\end{array}$ & $\begin{array}{l}\text { C3: RLEN < } 0 \\
\text { C4: RLEN is greater than the } \\
\text { length of String }\end{array}$ & \\
\hline $\begin{array}{l}\text { Category } 3 \text { : Contains } \\
\text { choices for all valid } \\
\text { values for ODD_DIGIT }\end{array}$ & $\begin{array}{l}\text { C5: Odd_digit }=-1 \\
\text { C6: Odd_digit is between } 0 \text { and } 9 \\
\text { C7: Odd_digit is between } a \text { and } f \\
\text { C8: Odd_digit is between } A \text { and } F\end{array}$ & \multirow[b]{2}{*}{ ODD_DIGIT } \\
\hline $\begin{array}{l}\text { Category } 4: \text { Contains } \\
\text { choices for all } \\
\text { invalid values for } \\
\text { ODD DIGIT }\end{array}$ & $\begin{array}{l}\text { C9: Odd_digit is less than } 0 \\
\text { c10: Odd_digit is greater than } 15\end{array}$ & \\
\hline $\begin{array}{l}\text { Category 5: Contains } \\
\text { choices for the length } \\
\text { of the string } \\
\end{array}$ & $\begin{array}{l}\text { C11: String is empty } \\
\text { C12: String is not empty }\end{array}$ & S LENGTH \\
\hline $\begin{array}{l}\text { Category } 6 \text { : Contains } \\
\text { choices for the type } \\
\text { of the first rlen } \\
\text { character in the } \\
\text { string } S \text { (Applies only } \\
\text { when } C 2 \text { or C4 holds) }\end{array}$ & $\begin{array}{l}\text { C13: All rlen characters are } \\
\text { hexadecimal } \\
\text { C14: All rlen characters are non } \\
\text { hexadecimal } \\
\text { C15: rlen characters are a mix of } \\
\text { hexadecimal and non hexadecimal } \\
\text { characters }\end{array}$ & $\begin{array}{l}\text { S CHAR TYPE } \\
\text { IN } \\
{[0 \ldots \text { RLEN] }}\end{array}$ \\
\hline $\begin{array}{l}\text { CATEGORY } 7: \text { Case of } \\
\text { chars in the first } \\
\text { rlen chars of the } \\
\text { string } s\end{array}$ & $\begin{array}{l}\text { C16: First rlen characters of the } \\
\text { string } S \text { are all numbers } \\
\text { C17: First rlen characters of the } \\
\text { string S are all lower case } \\
\text { letters } \\
\text { C18: First rlen characters of the } \\
\text { string S are all upper case } \\
\text { letters } \\
\text { C19: First rlen characters of the } \\
\text { string S is a mix of numbers, } \\
\text { lower case letters and upper case } \\
\text { letters ( combination of either } \\
\text { C16 and C17 or C16 and C18 or C17 } \\
\text { and C18 or C16, C17 and C18) }\end{array}$ & $\begin{array}{l}\text { CHAR CASE } \\
\text { IN } \\
{[0 . \text { RLEN] }}\end{array}$ \\
\hline $\begin{array}{l}\text { CATEGORY } 8-\text { The first } \\
\text { rlen characters of the } \\
\text { string contains } \\
\text { boundary value }[0,9, \\
\text { a, } f, A, F]\end{array}$ & $\begin{array}{l}\text { C20: The first rlen chars of the } \\
\text { string S contain } 0 \text { but no other } \\
\text { boundary value. } \\
\text { C21: The first rlen chars of the } \\
\text { string S contain } 9 \text { but no other } \\
\text { boundary value. } \\
\text { C22: The first rlen chars of the } \\
\text { string S contain "a" but no other } \\
\text { boundary value. } \\
\text { C23: The first rlen chars of the } \\
\text { string s contain f but no other } \\
\text { boundary value. } \\
\text { C24: The first rlen chars of the } \\
\text { string S contain A, but no other } \\
\text { boundary value. } \\
\text { C25: The first rlen chars of the }\end{array}$ & $\begin{array}{l}\text { BOUNDARY } \\
\text { VALUE IN } \\
{[0 . . \text { RLEN] }}\end{array}$ \\
\hline
\end{tabular}




\begin{tabular}{|c|c|c|}
\hline & $\begin{array}{l}\text { string } S \text { contain } F \text { but no other } \\
\text { boundary value. } \\
\text { C26: The first rlen chars of the } \\
\text { string S contain a mix of (either } \\
\text { all or some) of the boundary } \\
\text { values } \\
\text { C27: The first rlen chars of the } \\
\text { string S contain no boundary value }\end{array}$ & \\
\hline $\begin{array}{l}\text { Category } 9 \text { : Contains } \\
\text { choices for whether } \\
\text { the number of } \\
\text { hexadecimal characters } \\
\text { in the first rlen } \\
\text { characters of the } \\
\text { String } S \text { is Odd, Even } \\
\text { or zero. This category } \\
\text { holds if rlen not } 0 .\end{array}$ & $\begin{array}{l}\text { C28: Number of hexadecimal } \\
\text { characters in the first rlen } \\
\text { characters of the String } S \text { is odd } \\
\text { C29: Number of hexadecimal } \\
\text { characters in the first rlen } \\
\text { characters of the String } S \text { is Even } \\
\text { C30: Number of hexadecimal } \\
\text { characters in the first rlen } \\
\text { characters of the String } S \text { is zero }\end{array}$ & $\begin{array}{l}\text { NUM HEX } \\
\text { CHARS IN } \\
\text { [0...RLEN] }\end{array}$ \\
\hline $\begin{array}{l}\text { Category } 10 \text { : Contains } \\
\text { value for the position } \\
\text { of the first non- } \\
\text { hexadecimal character } \\
\text { in the first rlen } \\
\text { characters of S. This } \\
\text { category only applies } \\
\text { if c12 and C15 hold. }\end{array}$ & $\begin{array}{l}\text { C31: The position of the first non } \\
\text { hexadecimal character is in the } \\
\text { first position of the first rlen } \\
\text { characters of the string } \mathrm{s} \text {. } \\
\text { C32: The position of the first non } \\
\text { hexadecimal character is in the } \\
\text { middle of the first rlen } \\
\text { characters of the string s. } \\
\text { C33: The position of the first non } \\
\text { hexadecimal character is in the } \\
\text { last position of the first rlen } \\
\text { characters of the string s. }\end{array}$ & $\begin{array}{l}\text { NON HEX } \\
\text { CHAR } \\
\text { POSTION IN } \\
{[0 . \text { RLEN] }}\end{array}$ \\
\hline $\begin{array}{l}\text { Category 11: This } \\
\text { category contains } \\
\text { choices for whether } \\
\text { there are consecutive } \\
\text { non-hexadecimal } \\
\text { characters in the } \\
\text { first rlen characters } \\
\text { of } \mathrm{S} \text {. Applies if C3 } \\
\text { does not hold. }\end{array}$ & $\begin{array}{l}\text { C34: There are consecutive non } \\
\text { hexadecimal characters in the } \\
\text { first rlen characters of the } \\
\text { string } \mathrm{s} \text {. } \\
\text { C35: There are no consecutive non } \\
\text { hexadecimal characters in the } \\
\text { first rlen characters of the } \\
\text { string } \mathrm{s} \text {. }\end{array}$ & $\begin{array}{l}\text { CONSECUTIVE } \\
\text { NON HEX } \\
\text { CHARS IN } \\
{[0 . . \text { RLEN ] }}\end{array}$ \\
\hline
\end{tabular}




\section{A.3 Student A: CP Specification}

Parameter RLEN

Category 1: In Range

Choice 1: $\{0\}$

Choice 2: Length of $\mathrm{S}-1$

Category 2: Out of Range

Choice 3: Negative

Choice 4: Length of S

[if not OutOfRange]
[if not OutOfRange]

[Property OutOfRangeNeg] [Error]

[Property OutOfRangePos] [Error]

Parameter ODD_DIGIT

Category 3: No Ödd Digit

Choice 5: $\{-1\}$

Choice 6: [0-15]

Category 4: Out of Range

Choice 7: $\{-2\}$

Choice 8: $\{16\}$

[Property OddDigit] [Single]

[if not OutOfRange] [if not OddDigit]

[Error]

[Property OutOfRange] [Error]

Parameter S

Category 5: Length

Choice 9: Odd

Choice 10: Even

Choice 11: Empty

Category 6: Valid or Invalid Characters

Choice 12: Valid

Choice 13: Invalid (By Invalid the student means Mixe of valid and invalid)

//This category is added to fix student A's CP

Category 7: Number of valid hex characters from 0 to rlen

Choice 14: Odd

Choice 15: Even

Choice 16: Zero 


\section{A.4 Student B: CP Specifications}

Parameter: rlen

Category 1: Legal

Choice 1: 0

Choice 2: $<$ S

Category 2: Not Legal

Choice 3: $<0$

Choice 4: $>$ S
[Property noCharToPack, isLegal] [single]

[Property isLegal]

[Property not isLegal] [error]

[error] [Property not isLegal] [error]

[Property digitIsLegal]

[Property digitIsLegal]

[Property not digitIsLegal] [error]

[Property not digitIsLegal] [error]

Parameter: string

Category 5: Case of characters

Choice 9: uppercase

[if not noCharInString] [single]

Choice 10: uppercase \& lowercase [if not noCharInString]

Choice 11: lowercase

[if not noCharInString]

Category 6: Type of characters

Choice 12: valid

[if not noCharInString]

Choice 13: valid \& invalid [if not noCharInString]

Choice 14: invalid

Category 7: length of string

Choice 15: zero

[if not noCharInString] [single]

Choice 16: < rlen

[Property noCharInString]

Choice 17: $=$ rlen

[error] [if not noCharToPack]

Choice 18: > rlen

Category 8: \# characters in string

Choice 19: even

Choice 20: odd

//This category is added to fix student A's CP

Category 9: Number of valid hex characters from 0 to rlen

Choice 21: Odd

Choice 22: Even

Choice 23: Zero 


\section{A.5 Student C: CP Specifications}

Parameter: rlen

Category 1: number of characters to read

Choice 1: $\{0\}$ [property readNone]

Choice 2: -1 - s.length] [property readSome]

Choice 3: anything else [property readTooMany][error]

Parameter: odd_digit

Category 2: Extra digit to parse

Choice 4: $\{-1\}$

Choice 5: -0 - 15]

Choice 6: anything else [error]

Parameter: $s$

Category 3: length of string

Choice 7: $\{0\}$

[if readNone and if not readSome and if not readTooMany]

Choice 8: anything else

[if not readTooMany]

Category 4: number of invalid characters

Choice 9: none

[if not readNone and if not readTooMany]

Choice 10: several

[if not readNone and if not readTooMany]

Choice 11: maximum

[if not readNone and if not readTooMany][single]

//This category is added to fix student A's CP

Category 5: Number of valid hex characters from 0 to rlen

Choice 12: Odd

Choice 13: Even

Choice 14: Zero 


\section{Appendix B Mapping of Categories/Choices of Expert CP and Student CPs}

The tuple $\left(\mathrm{cat}_{\mathrm{i}}, \mathrm{ch}_{\mathrm{i}}\right)$ represents a choice $\mathrm{ch}_{\mathrm{i}}$ of category cat $\mathrm{t}_{\mathrm{i}}$. The three table below represents Categories and choices in the $\mathrm{CP}_{\text {sub }} \mathrm{s}$. Studetn $\mathrm{A}$, and B's $\mathrm{CP}_{\mathrm{sub}} \mathrm{s}$ can be compared to the Expert $\mathrm{CP}_{\text {sub }}$ in the same way as student $\mathrm{C}^{\prime} \mathrm{SCP}_{\text {sub }}$ was compared to the expert $\mathrm{CP}_{\text {sub }}$ in section 5.4.1.

\begin{tabular}{|c|c|c|}
\hline Expert CP & Relation & Student $\mathbf{A}$ \\
\hline (Cat 1, Ch1) & $=$ & (Cat1, Ch1) \\
\hline (Cat1, Ch2) & $=$ & (Cat1, Ch2) \\
\hline (Cat2, Ch3) & $=$ & (Cat2, Ch3) \\
\hline (Cat2, Ch4) & $=$ & $(\operatorname{Cat} 2, \operatorname{Ch} 4)$ \\
\hline (Cat3, Ch5) & $=$ & $($ Cat 3, Ch5) \\
\hline (Cat 3, Ch6) & $\subset$ & $(\operatorname{Cat} 3, \operatorname{ch} 6)$ \\
\hline$(\operatorname{Cat} 3, \operatorname{Ch} 7)$ & $c$ & $(\operatorname{Cat} 3, \operatorname{ch} 6)$ \\
\hline (Cat3, Ch8) & $\bar{c}$ & (Cat3, Ch6) \\
\hline (Cat4, Ch9) & $=$ & (Cat4, Ch7) \\
\hline (Cat4, Ch10) & $=$ & (Cat4, Ch8) \\
\hline (Cat9, Ch28) & $=$ & (Cat7, Ch14) \\
\hline (Cat9, Ch29) & $=$ & (Cat7, Ch15) \\
\hline (Cat9, $\operatorname{ch} 30)$ & $=$ & $(\operatorname{Cat} 7, \operatorname{Ch} 16)$ \\
\hline
\end{tabular}

\begin{tabular}{|c|c|c|}
\hline Expert CP & Relation & Student B CP \\
\hline (Cat1, Ch1) & $=$ & (Cat1, Ch1) \\
\hline$(\operatorname{cat} 1, \operatorname{ch} 2)$ & $=$ & (Cat1, $\operatorname{ch} 2)$ \\
\hline$(\operatorname{cat} 2, \operatorname{ch} 3)$ & $=$ & $(\operatorname{Cat} 2, \operatorname{Ch} 3)$ \\
\hline$(\operatorname{cat} 2, \operatorname{ch} 4)$ & $=$ & $(\operatorname{Cat} 2, \operatorname{Ch} 4)$ \\
\hline$(\operatorname{cat} 3, \operatorname{ch} 5)$ & $=$ & $(\operatorname{Cat} 3, \operatorname{ch} 5)$ \\
\hline$(\operatorname{cat} 3, \operatorname{ch} 6)$ & $\subset$ & (Cat3, Ch6) \\
\hline$(\operatorname{Cat} 3, \operatorname{Ch} 7)$ & $\subset$ & (Cat3, Ch6) \\
\hline (Cat $3, \operatorname{ch} 8)$ & $\subset$ & (Cat $3, \operatorname{ch} 6)$ \\
\hline$(\operatorname{Cat} 4, \operatorname{Ch} 9)$ & $=$ & $(\operatorname{Cat} 4, \operatorname{Ch} 7)$ \\
\hline (Cat 4, Ch10) & $=$ & (Cat $4, \operatorname{Ch} 8)$ \\
\hline (Cat9, $\operatorname{ch} 28)$ & $=$ & (Cat9, Ch14) \\
\hline (Cat9, Ch29) & $=$ & (Cat9, Ch15) \\
\hline$(\operatorname{cat} 9, \operatorname{ch} 30)$ & $=$ & (Cat9, Ch16) \\
\hline
\end{tabular}




\begin{tabular}{|c|c|c|}
\hline Expert CP & Relation & Student C CP \\
\hline$(\operatorname{Cat} 1, \operatorname{Ch} 1)$ & $=$ & (Cat1, Ch1) \\
\hline (Cat1, Ch2) & $=$ & (Cat1, Ch2) \\
\hline (Cat2, Ch3) & $c$ & (Cat1, Ch3) \\
\hline$($ Cat 2, Ch 4$)$ & $c$ & (Cat1, Ch3) \\
\hline$(\operatorname{Cat} 3$, Ch5) & $=$ & $(\operatorname{cat} 2, \operatorname{ch} 4)$ \\
\hline$(\operatorname{Cat} 3$, Ch6) & $\subset$ & (Cat2, Ch5) \\
\hline$(\operatorname{Cat} 3, \operatorname{Ch} 7)$ & $c$ & (Cat2, Ch5) \\
\hline (Cat3, Ch8) & $c$ & (Cat2, Ch5) \\
\hline (Cat4, Ch9) & $c$ & (Cat2, Ch6) \\
\hline$(\operatorname{Cat} 4, \operatorname{Ch} 10)$ & $\subset$ & (Cat2, Ch6) \\
\hline (Cat9, Ch28) & $=$ & $(\operatorname{Cat} 5, \operatorname{Ch} 12)$ \\
\hline (Cat9, Ch29) & $=$ & (Cat5, Ch13) \\
\hline$($ Cat9, $\operatorname{Ch} 30)$ & $=$ & (Cat5, Ch14) \\
\hline
\end{tabular}




\section{Appendix C PackHexChar: Decision Trees before TS Reduction}

\section{C.1 Expert CP and Expert TS}

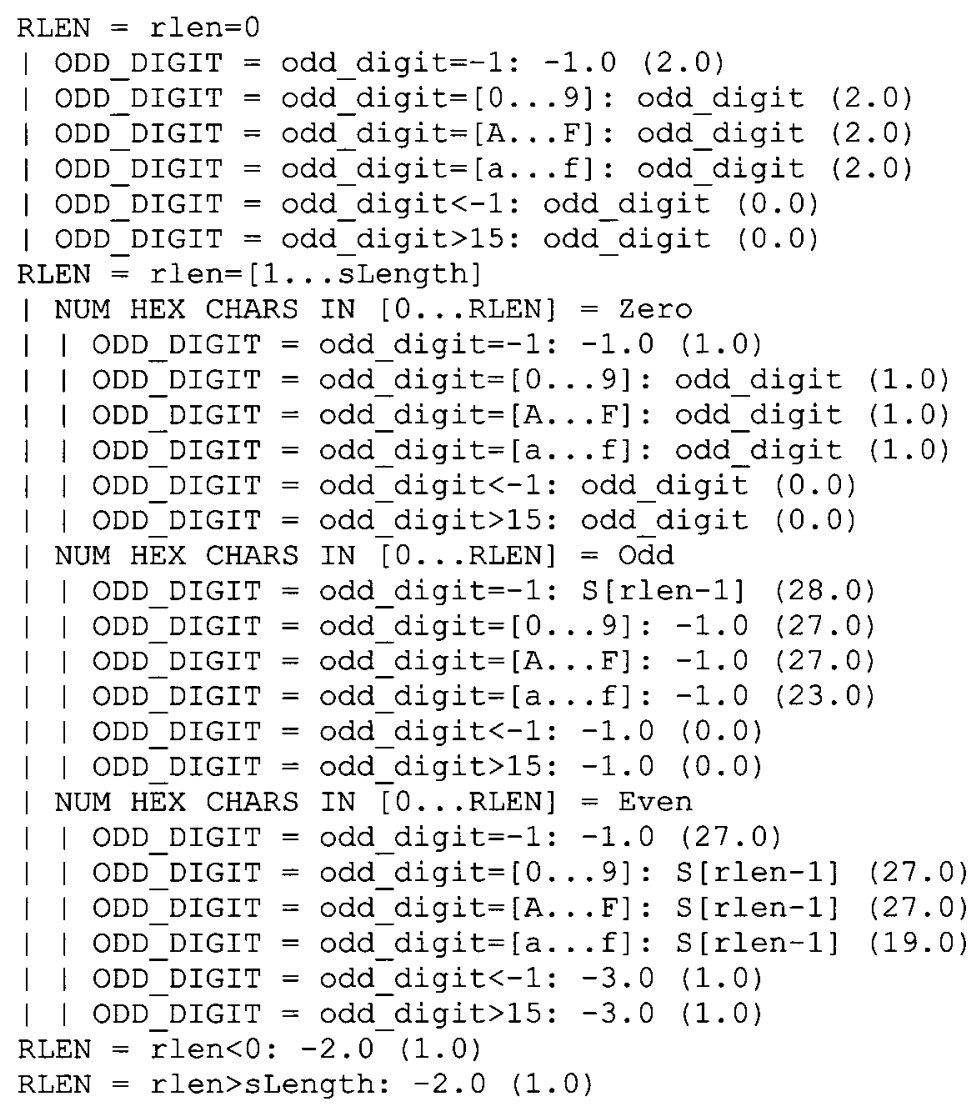




\section{C.2 Expert CP and Student X TS (Iteration 3)}

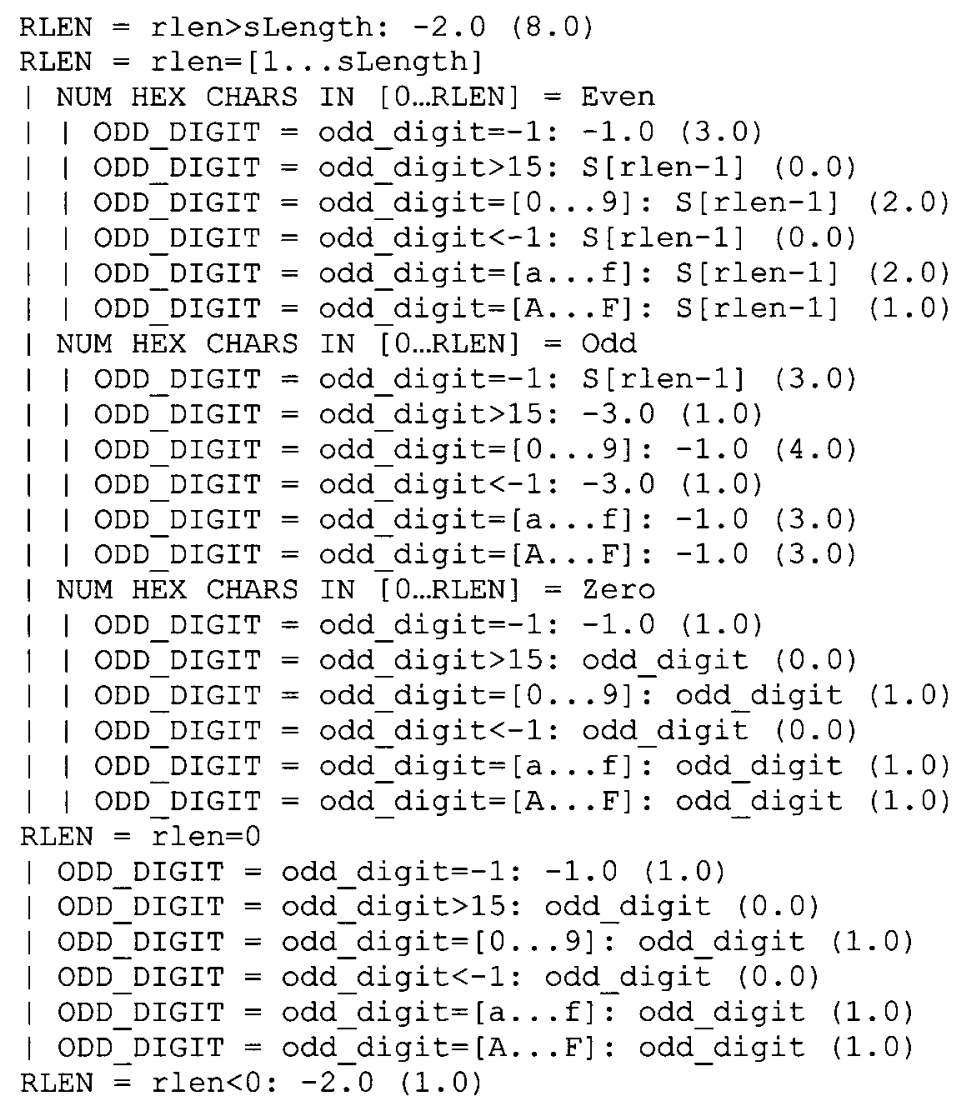




\section{C.3 Expert CP and Student Y TS (Iteration 3)}

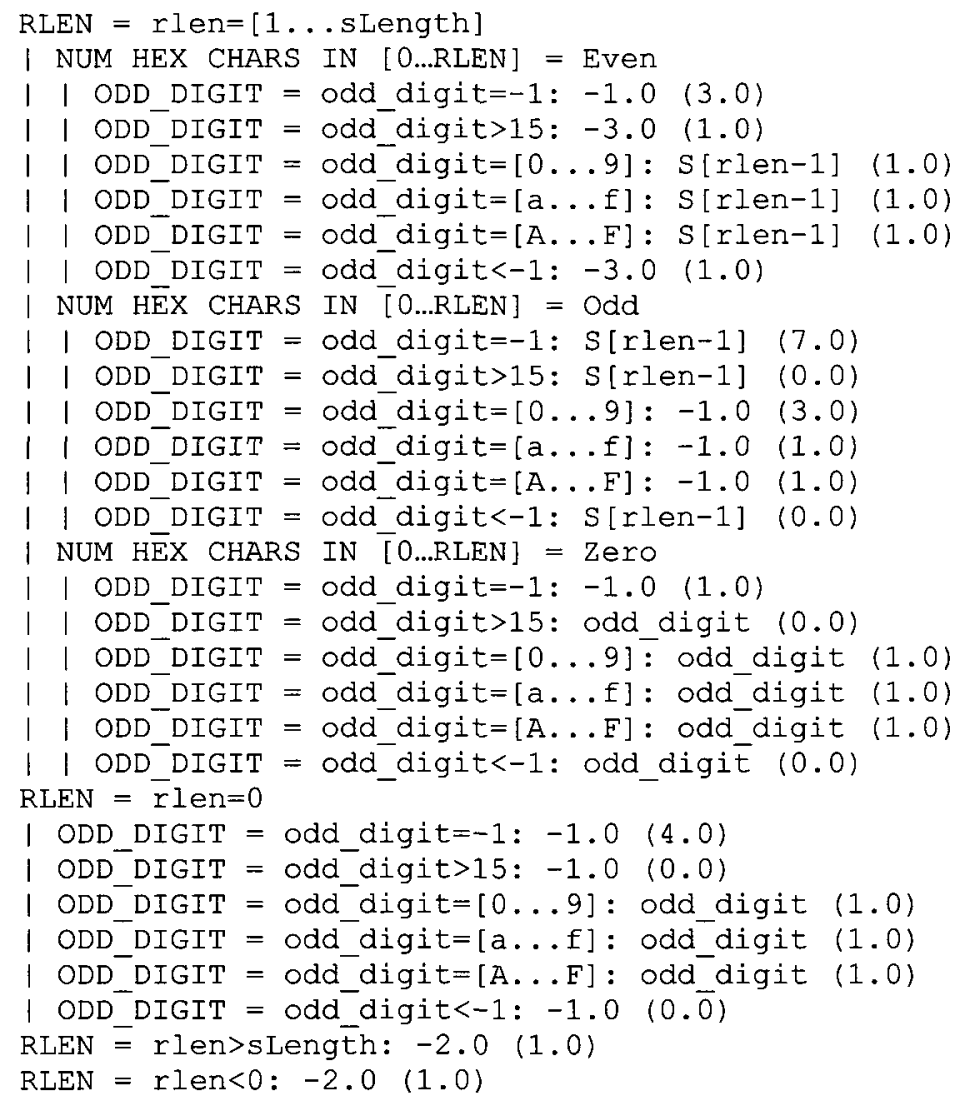




\section{C.4 Expert CP and Student Z TS (Iteration 3)}

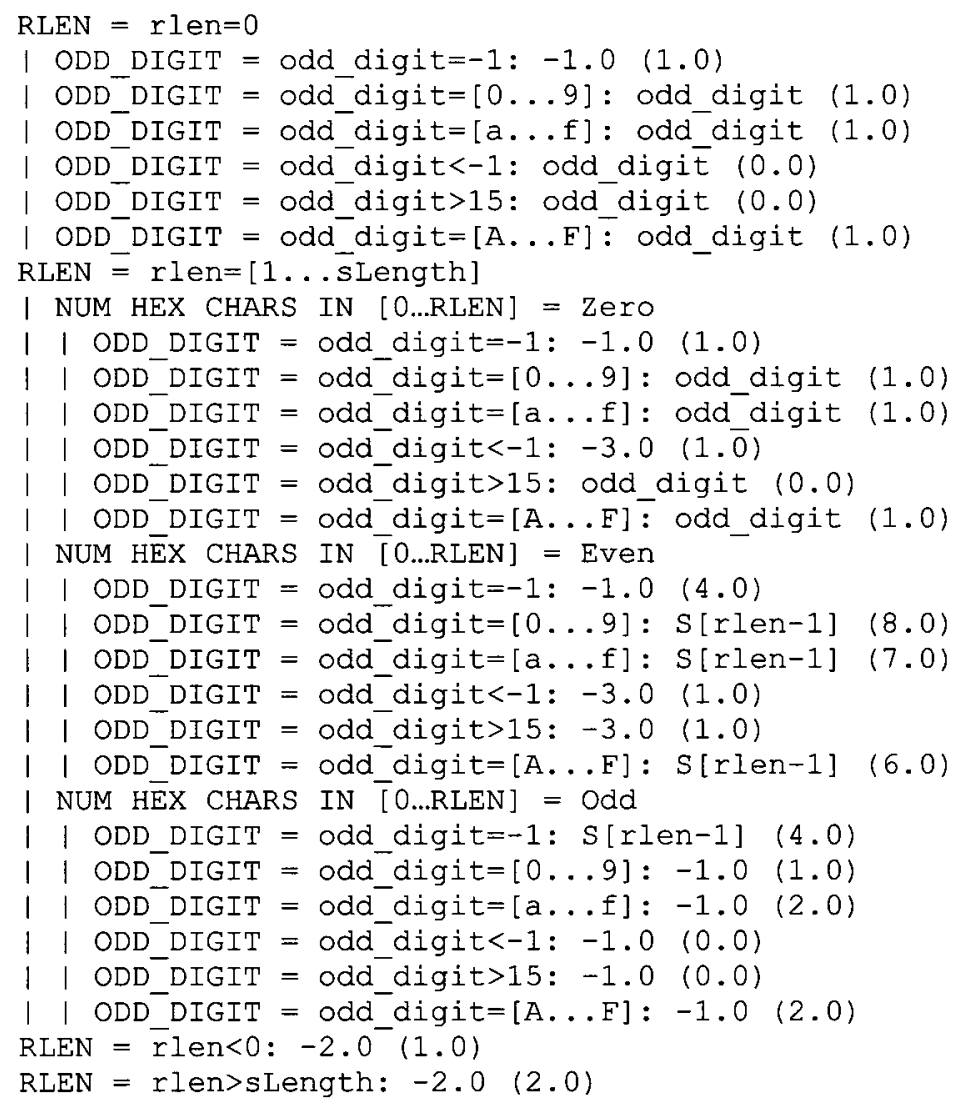

\section{C.5 Student A CP and TS (Iteration 3)}

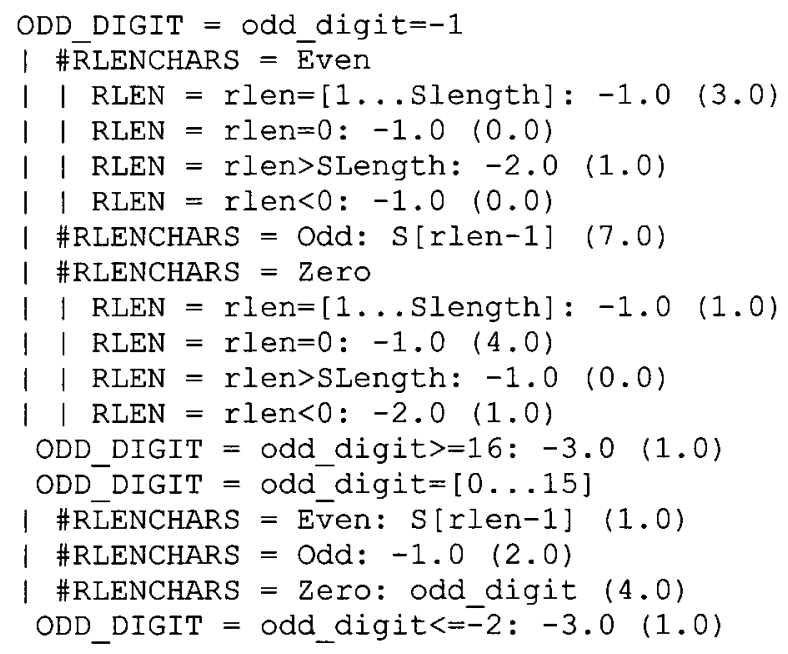




\section{C.6 Student B CP and TS (Iteration 3)}

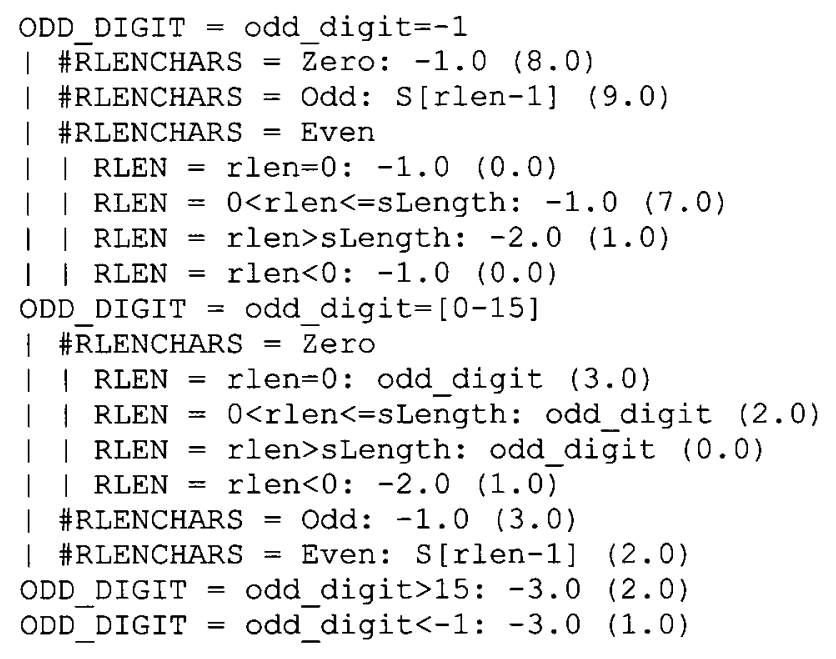

\section{C.7 Student C CP and TS (Iteration 3)}

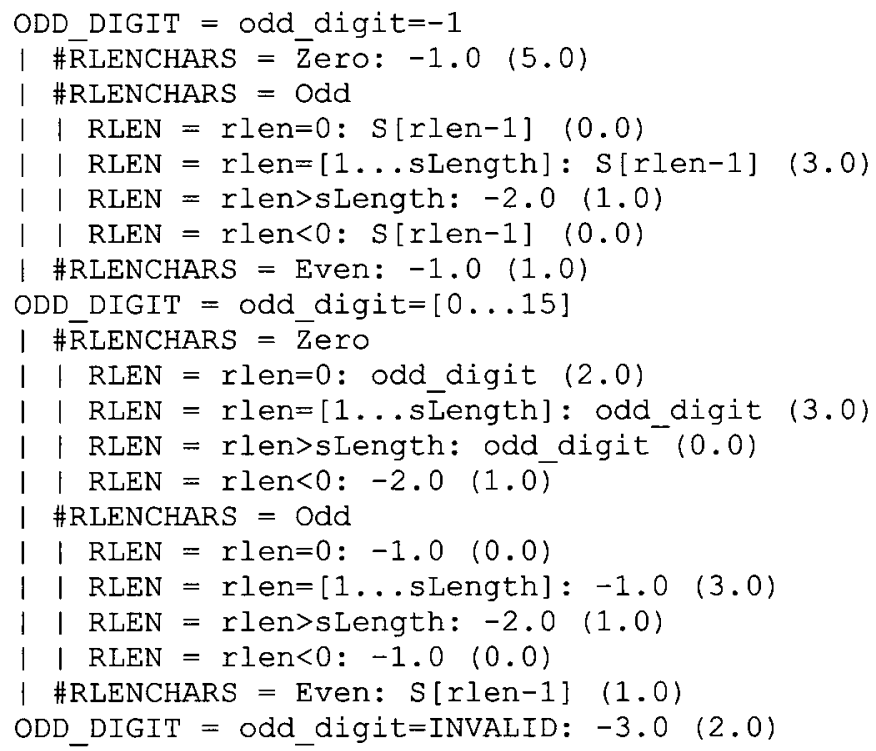




\section{Appendix D Mutation Results-PackHexChar Program}

\begin{tabular}{|c|c|c|}
\hline Student ID & Test Suite ID & Mutant ID (Not killed) \\
\hline \multirow{2}{*}{ (6) } & Iteration 3 & ROR11, ROR18, ROR21, ROR28 \\
\hline & Iteration 4 & AOIU3, AORB14, ROR11, ROR18, ROR21, ROR28 \\
\hline \multirow{2}{*}{ Y } & Iteration 3 & AOIS102, ROR8 ROR18, ROR28 \\
\hline & Iteration 4 & AOIS102, ROR8, ROR14, ROR18, ROR28 \\
\hline \multirow{2}{*}{$Z$} & Iteration 3 & AOIS102 \\
\hline & Iteration 4 & AOIS102 \\
\hline \multirow[b]{2}{*}{ A } & Iteration 3 & AOIS102, ROR1, ROR8, ROR18,ROR28 \\
\hline & Iteration 4 & $\begin{array}{l}\text { AOIS102, ROR1, ROR5, ROR8, ROR13, ROR14, ROR18, } \\
\text { ROR28, ROR36 }\end{array}$ \\
\hline \multirow{2}{*}{ B } & Iteration 3 & $\begin{array}{l}\text { AOIS102, AOIS37, AOIS39, AOIS40, AOIS41, AOIS42, AOIS43, } \\
\text { AOIS44, AOIU3, AORB13, AORB14, AORB16, AORB17, } \\
\text { AORB18, AORB19, AORB20, LOI12, ROR8, ROR18, ROR21, } \\
\text { ROR24, ROR28, ROR29, ROR36 }\end{array}$ \\
\hline & Iteration 4 & $\begin{array}{l}\text { OIS102, AOIS31, AOIS37, AOIS39, AOIS40, AOIS41, AOIS42, } \\
\text { AOIS43, AOIS44, AOIU3, AORB13, AORB14, AORB16, } \\
\text { AORB17, AORB18, AORB19, AORB20, LOI12, ROR18, ROR8, } \\
\text { ROR21, ROR24, ROR28, ROR29, ROR31, ROR36 }\end{array}$ \\
\hline \multirow{2}{*}{$\mathrm{C}$} & Iteration 3 & $\begin{array}{l}\text { AOIS37, AOIS38, AOIS39, AOIS40, AOIS41, AOIS42, AOIS43, } \\
\text { AOIS44, AOIU3, AORB13, AORB14, AORB16, AORB17, } \\
\text { AORB18, AORB19, AORB20, COI9, LOI11, LOI12, ROR8, } \\
\text { ROR18, ROR21, ROR24, ROR26, ROR27, ROR28, ROR29, } \\
\text { ROR3, ROR30, ROR36, ROR4 }\end{array}$ \\
\hline & Iteration 4 & $\begin{array}{l}\text { AOIS37, AOIS38, AOIS39, AOIS40, AOIS41, AOIS42, AOIS43, } \\
\text { AOIS44, AOIU3, AORB13, AORB14, AORB16, AORB17, } \\
\text { AORB18, AORB19, AORB20, COI9, LOI11, LOI12, ROR8, } \\
\text { ROR18, ROR21, ROR24, ROR26, ROR27, ROR28, ROR29, } \\
\text { ROR3, ROR30, ROR36, ROR4 }\end{array}$ \\
\hline \multirow{2}{*}{$\begin{array}{l}\text { Our } \\
\text { CP+TS }\end{array}$} & Iteration 1 & $\mathrm{~N} / \mathrm{A}$ \\
\hline & Iteration 2 & $\begin{array}{l}\text { AOIS102, ROR1, ROR11, ROR18, ROR21, ROR28, ROR31, } \\
\text { ROR8 }\end{array}$ \\
\hline
\end{tabular}

Table 6 Mutants not killed in the last two iterations for all students

\begin{tabular}{|l|l|l|}
\hline $\begin{array}{l}\text { Mutant } \\
\text { Name }\end{array}$ & Mutation Operator & How to Kill? \\
\hline AOIU3 & $+\rightarrow-$ (sign change) & $\begin{array}{l}\text { The first rlen characters of the string S must contain } \\
\text { one or more hex char between [a...f] but not at the } \\
0^{\text {th }} \text { index. e.g."0al" }\end{array}$ \\
\hline AORB14 & $-\rightarrow /$ & $\begin{array}{l}\text { The first rlen characters of the string S must contain } \\
\text { a hex char between [a...f] other than b. e.g. "a31" }\end{array}$ \\
\hline
\end{tabular}

Table 7 Mutants not killed by reduced student X TS 


\begin{tabular}{|l|l|l|}
\hline Mutant Name & Mutation Operator & How to Kill? \\
\hline ROR14 & $>=\rightarrow==$ & $\begin{array}{l}\text { The first rlen characters of the string S must contain } \\
\text { one or more hex chars between [B...F]. }\end{array}$ \\
\hline ROR18 & $<=\rightarrow<$ & $\begin{array}{l}\text { The first rlen characters of the string S must contain } \\
\text { F. }\end{array}$ \\
\hline
\end{tabular}

Table 8 Mutants not killed by reduced student Y TS

\begin{tabular}{|l|l|l|}
\hline Mutant Name & Mutation Operator & How to Kill? \\
\hline AOIS102 & Post decrement(--) & $\begin{array}{l}\text { The number of hex chars in the first rlen } \\
\text { characters of the string is more than one where } \\
\text { the last character is 0. }\end{array}$ \\
\hline
\end{tabular}

Table 9 Mutants not killed by reduced student $Z$ TS

\begin{tabular}{|l|l|l|}
\hline Mutant Name & Mutation Operator & How to Kill? \\
\hline ROR5 & $>=\rightarrow !=$ & $\begin{array}{l}\text { The first rlen characters of the string S must contain } \\
\text { one or more characters whose value is less than } 48 \\
(0)\end{array}$ \\
\hline ROR13 & $>=\rightarrow<=$ & $\begin{array}{l}\text { The first rlen characters of the string S must contain } \\
\text { one or more characters whose value is less than } 65 \\
\text { (A) and is not between [0...9] }\end{array}$ \\
\hline ROR14 & $>=\rightarrow==$ & $\begin{array}{l}\text { The first rlen characters of the string S must contain } \\
\text { one or more hex chars between [B...F] }\end{array}$ \\
\hline ROR36 & $>\rightarrow<=$ & Odd digit must have a value 15. \\
\hline
\end{tabular}

Table 10 Mutants not killed by reduced student A TS 


\begin{tabular}{|c|c|c|}
\hline Mutant Name & Mutation Operator & How to Kill? \\
\hline AOIS37 & Pre-Increment $(++)$ & $\begin{array}{l}\text { The first rlen characters of the string } \mathrm{S} \text { contains one or } \\
\text { more characters in [a...f], and if the last letter in the first } \\
\text { rlen characters of the Sring } \mathrm{S} \text { is between [a...f] then the } \\
\text { following letter should not be the same one. }\end{array}$ \\
\hline AOIS39 & Post-Incremen $(++)$ & $\begin{array}{l}\text { The first rlen characters of the string } S \text { contains one or } \\
\text { more characters in }[a \ldots f] \text {, and if the last letter in the first } \\
\text { rlen characters of the Sring } S \text { is between }[a \ldots f] \text { then the } \\
\text { following letter should not be the same one. }\end{array}$ \\
\hline AOIS40 & Post-Decremen(--) & $\begin{array}{l}\text { The first rlen characters of the string } \mathrm{S} \text { contains one or } \\
\text { more characters in }[\mathrm{a} \ldots \mathrm{f}]\end{array}$ \\
\hline AOIS41 & Pre-Increment $(++)$ & $\begin{array}{l}\text { The first rlen characters of the string } \mathrm{S} \text { contains one or } \\
\text { more characters in [a...f], and if the last letter in the first } \\
\text { rlen characters of the Sring } \mathrm{S} \text { is between [a...f] then the } \\
\text { following letter should not be the same one. }\end{array}$ \\
\hline AOIS42 & Pre-Decrement(--) & $\begin{array}{l}\text { The first rlen characters of the string } \mathrm{S} \text { contains one or } \\
\text { more characters in [a...f] }\end{array}$ \\
\hline AOIS43 & Post-Increment(++) & $\begin{array}{l}\text { The first rlen characters of the string } S \text { contains one or } \\
\text { more characters in [a...f], and if the last letter in the first } \\
\text { rlen characters of the Sring } S \text { is between [a...f] then the } \\
\text { following letter should not be the same one. }\end{array}$ \\
\hline \multicolumn{3}{|r|}{ 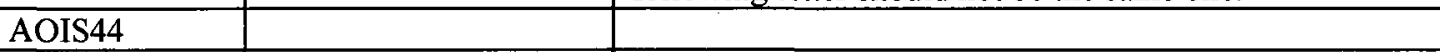 } \\
\hline AOIS102 & Post decrement(--) & $\begin{array}{l}\text { The number of hex chars in the first rlen characters of the } \\
\text { string is more than one where the last character is } 0 \text {. }\end{array}$ \\
\hline AOIU3 & $+\rightarrow-$ & $\begin{array}{l}\text { The first rlen characters of the string S contains one or } \\
\text { more characters in [a...f] anywhere except at index } 0\end{array}$ \\
\hline AORB13 & $-\rightarrow *$ & $\begin{array}{l}\text { The first rlen characters of the string S contains one or } \\
\text { more characters in [a...f] }\end{array}$ \\
\hline AORB14 & $-\rightarrow 1$ & The first rlen characters of the string $S$ contains the char a. \\
\hline AORB 16 & $-\rightarrow+$ & $\begin{array}{l}\text { The first rlen characters of the string } S \text { contains one or } \\
\text { more characters in }[\mathrm{a} \ldots \mathrm{f}]\end{array}$ \\
\hline AORB 17 & $+\rightarrow *$ & $\begin{array}{l}\text { The first rlen characters of the string S contains one or } \\
\text { more characters in [a...f] }\end{array}$ \\
\hline AORB18 & $+\rightarrow 1$ & $\begin{array}{l}\text { The first rlen characters of the string S contains one or } \\
\text { more characters in [a...f] }\end{array}$ \\
\hline AORB 19 & $+\rightarrow \%$ & $\begin{array}{l}\text { The first rlen characters of the string S contains one or } \\
\text { more characters in [a...f] }\end{array}$ \\
\hline AORB20 & $+\rightarrow-$ & $\begin{array}{l}\text { The first rlen characters of the string } \mathrm{S} \text { contains one or } \\
\text { more characters in [a...f] }\end{array}$ \\
\hline ROR8 & $<=\rightarrow<$ & The first rlen characters of the string S must contain 9 . \\
\hline ROR18 & $<=\rightarrow<$ & The first rlen characters of the string S must contain F. \\
\hline ROR21 & $>=\rightarrow>$ & The first rlen characters of the string S must contain a. \\
\hline ROR24 & $>=\rightarrow==$ & $\begin{array}{l}\text { The first rlen characters of the string S contains one or } \\
\text { more characters in [b...f] }\end{array}$ \\
\hline ROR28 & $<=\rightarrow<$ & The first rlen characters of the string S must contain $\mathrm{F}$. \\
\hline ROR29 & $<=\rightarrow==$ & $\begin{array}{l}\text { The first rlen characters of the string S contains one or } \\
\text { more characters in [a...e] }\end{array}$ \\
\hline ROR36 & & \\
\hline
\end{tabular}

Table 11 Mutants not killed by reduced student B TS 


\begin{tabular}{|c|c|c|}
\hline Mutant Name & Mutation Operator & How to Kill? \\
\hline AOIS37 & Pre-Increment $(++)$ & $\begin{array}{l}\text { The first rlen characters of the string } S \text { contains one } \\
\text { or more characters in [a...f], and if the last letter in } \\
\text { the first rlen characters of the Sring } S \text { is between } \\
{[\text { a...f] then the following letter should not be the }} \\
\text { same one. }\end{array}$ \\
\hline AOIS38 & Pre-Decrement (--) & $\begin{array}{l}\text { The first rlen characters of the string S contains one } \\
\text { or more characters in }[\mathrm{a} . . \mathrm{f}]\end{array}$ \\
\hline AOIS39 & Post-Incremen $(++)$ & $\begin{array}{l}\text { The first rlen characters of the string S contains one } \\
\text { or more characters in [a...f], and if the last letter in } \\
\text { the first rlen characters of the Sring } S \text { is between } \\
\text { [a...f] then the following letter should not be the } \\
\text { same one. }\end{array}$ \\
\hline AOIS40 & Post-Decremen(--) & $\begin{array}{l}\text { The first rlen characters of the string } S \text { contains one } \\
\text { or more characters in }[\mathrm{a} \ldots \mathrm{f}]\end{array}$ \\
\hline AOIS41 & Pre-Increment $(++)$ & $\begin{array}{l}\text { The first rlen characters of the string S contains one } \\
\text { or more characters in [a...f], and if the last letter in } \\
\text { the first rlen characters of the Sring } S \text { is between } \\
\text { [a...f] then the following letter should not be the } \\
\text { same one. }\end{array}$ \\
\hline AOIS42 & Pre-Decrement(--) & $\begin{array}{l}\text { The first rlen characters of the string S contains one } \\
\text { or more characters in }[\mathrm{a} . . . \mathrm{f}]\end{array}$ \\
\hline AOIS43 & Post-Increment(+) & $\begin{array}{l}\text { The first rlen characters of the string S contains one } \\
\text { or more characters in [a...f], and if the last letter in } \\
\text { the first rlen characters of the Sring } S \text { is between } \\
\text { [a...f] then the following letter should not be the } \\
\text { same one. }\end{array}$ \\
\hline AOIU3 & $+\rightarrow$ & $\begin{array}{l}\text { The first rlen characters of the string S contains one } \\
\text { or more characters in [a...f] anywhere except at } \\
\text { index } 0\end{array}$ \\
\hline AORB13 & $-\rightarrow *$ & $\begin{array}{l}\text { The first rlen characters of the string S contains one } \\
\text { or more characters in }[\mathrm{a} \ldots \mathrm{f}]\end{array}$ \\
\hline AORB14 & $-\rightarrow 1$ & $\begin{array}{l}\text { The first rlen characters of the string } \mathrm{S} \text { contains the } \\
\text { char a. }\end{array}$ \\
\hline AORB 16 & $-\rightarrow+$ & $\begin{array}{l}\text { The first rlen characters of the string S contains one } \\
\text { or more characters in }[\mathrm{a} . . . \mathrm{f}]\end{array}$ \\
\hline AORB 17 & $+\rightarrow *$ & $\begin{array}{l}\text { The first rlen characters of the string S contains one } \\
\text { or more characters in }[\mathrm{a} \ldots \mathrm{f}]\end{array}$ \\
\hline AORB 18 & $+\rightarrow 1$ & $\begin{array}{l}\text { The first rlen characters of the string } \mathrm{S} \text { contains one } \\
\text { or more characters in }[\mathrm{a} \ldots \mathrm{f}]\end{array}$ \\
\hline AORB 19 & $+\rightarrow \%$ & $\begin{array}{l}\text { The first rlen characters of the string } S \text { contains one } \\
\text { or more characters in }[\mathrm{a} \ldots \mathrm{f}]\end{array}$ \\
\hline AORB20 & $+\rightarrow-$ & $\begin{array}{l}\text { The first rlen characters of the string } S \text { contains one } \\
\text { or more characters in }[\mathrm{a} . . . \mathrm{f}]\end{array}$ \\
\hline COI9 & ! (Condtion Negated) & $\begin{array}{l}\text { The first rlen characters of the string S contains one } \\
\text { or more characters in }[\mathrm{a} \ldots \mathrm{f}]\end{array}$ \\
\hline LOI11 & $\sim$ & $\begin{array}{l}\text { The first rlen characters of the string } \mathrm{S} \text { contains one } \\
\text { or more characters in }[\mathrm{a} \ldots \mathrm{f}]\end{array}$ \\
\hline LOI12 & $\sim$ & $\begin{array}{l}\text { The first rlen characters of the string } S \text { contains one } \\
\text { or more characters in }[\mathrm{a} \ldots \mathrm{f}]\end{array}$ \\
\hline
\end{tabular}




\begin{tabular}{|l|l|l|}
\hline ROR3 & $>=\rightarrow<=$ & $\begin{array}{l}\text { The first rlen characters of the string S contains one } \\
\text { or more characters in [1..9] }\end{array}$ \\
\hline ROR4 & $>=\rightarrow==$ & $\begin{array}{l}\text { The first rlen characters of the string S contains one } \\
\text { or more characters in [1...9] }\end{array}$ \\
\hline ROR8 & $<=\rightarrow<$ & $\begin{array}{l}\text { The first rlen characters of the string S must contain } \\
9 .\end{array}$ \\
\hline ROR18 & $>=\rightarrow>$ & $\begin{array}{l}\text { The first rlen characters of the string S must contain } \\
\text { F. }\end{array}$ \\
\hline ROR21 & $\begin{array}{l}\text { The first rlen characters of the string S must contain } \\
\text { a. }\end{array}$ \\
\hline ROR24 & $>=\rightarrow==$ & $\begin{array}{l}\text { The first rlen characters of the string S contains one } \\
\text { or more characters in [b...f] }\end{array}$ \\
\hline ROR26 & $<=\rightarrow>$ & $\begin{array}{l}\text { The first rlen characters of the string S contains one } \\
\text { or more characters in [a...f] }\end{array}$ \\
\hline ROR27 & $<=\rightarrow>=$ & $\begin{array}{l}\text { The first rlen characters of the string S contains one } \\
\text { or more characters in [a...e] }\end{array}$ \\
\hline ROR28 & $<=\rightarrow<$ & $\begin{array}{l}\text { The first rlen characters of the string S must contain } \\
\text { F. }\end{array}$ \\
\hline ROR29 & $<=\rightarrow==$ & $\begin{array}{l}\text { The first rlen characters of the string S contains one } \\
\text { or more characters in [a...e] }\end{array}$ \\
\hline ROR30 & $<=\rightarrow !=$ & $\begin{array}{l}\text { The first rlen characters of the string S must contain } \\
\text { F. }\end{array}$ \\
\hline
\end{tabular}

Table 12 Mutants not killed by reduced student C TS

\begin{tabular}{|l|l|l|}
\hline Mutant Name & Mutation Operator & How to Kill? \\
\hline AOIS102 & -- ( decrement) & $\begin{array}{l}\text { The number of hex chars in the first rlen characters } \\
\text { of the string is more than one and the last character } \\
\text { is } 0\end{array}$ \\
\hline ROR1 & $>=\rightarrow>$ & $\begin{array}{l}\text { The first rlen characters of the string S must contain } \\
0 .\end{array}$ \\
\hline ROR8 & $<=\rightarrow<$ & $\begin{array}{l}\text { The first rlen characters of the string S must contain } \\
\text { 9. }\end{array}$ \\
\hline ROR11 & $>=\rightarrow>$ & $\begin{array}{l}\text { The first rlen characters of the string S must contain } \\
\text { A. }\end{array}$ \\
\hline ROR18 & $\langle=\rightarrow<$ & $\begin{array}{l}\text { The first rlen characters of the string S must contain } \\
\text { F. }\end{array}$ \\
\hline ROR21 & $>=\rightarrow>$ & $\begin{array}{l}\text { The first rlen characters of the string S must contain } \\
\text { a. }\end{array}$ \\
\hline ROR28 & $\langle=\rightarrow<$ & $\begin{array}{l}\text { The first rlen characters of the string S must contain } \\
\text { F. }\end{array}$ \\
\hline ROR31 & $>\rightarrow>=$ & $\begin{array}{l}\text { The first rlen characters of the string S must contain } \\
\text { f. }\end{array}$ \\
\hline AOIS31 & $\begin{array}{l}\text { The first rlen characters of the string S must contain } \\
\text { two or more consecutive hex chars with any but the } \\
\text { last between [A...F] }\end{array}$ \\
\hline
\end{tabular}

Table 13 Mutants not killed by reduced Expert TS 


\section{Appendix E The Space Program}

\section{E.1 CP Definition for the Space Program}

\begin{tabular}{|c|c|c|c|}
\hline POOO & Input & Parameter & \\
\hline & CA0001 & Valid Input & \\
\hline B & & CH000101 ValidFile & [property valid File] \\
\hline & & \begin{tabular}{l|l|} 
CH000102 & EmptyFile \\
\end{tabular} & [ERROR] \\
\hline & $\mathrm{CA} 0002$ & Invalid File & \\
\hline & & \begin{tabular}{|l|l|} 
CH000201 & Nonexist File \\
\end{tabular} & [ERROR] \\
\hline & & \begin{tabular}{l|l}
$\mathrm{CH} 000202$ & $\begin{array}{l}\text { Wrong_Parameter_N } \\
\text { um }\end{array}$ \\
\end{tabular} & [ERROR] \\
\hline PAO1 & Group & Name & \\
\hline & CA0101 & Length of name & \\
\hline & & CH010101 length $=0$ & [property no name] \\
\hline B & & \begin{tabular}{|l|l|}
$\mathrm{CH} 010102$ & length $[1 . .29]$ \\
\end{tabular} & [property gname] \\
\hline & & \begin{tabular}{|l|l|} 
CH010103 & length $>=30$ \\
\end{tabular} & [property too long gname] \\
\hline & CA0102 & Context of name & \\
\hline B & & \begin{tabular}{|l|l|l|l} 
CH020101 & start with Alpha \\
\end{tabular} & [if gname] \\
\hline & & \begin{tabular}{|l|l|}
$\mathrm{CH} 020102$ & start with Number \\
\end{tabular} & [if gname] \\
\hline & & CH020103 $\begin{array}{l}\text { start_with_other_ } \\
\text { Char }\end{array}$ & [if gname] \\
\hline & & \begin{tabular}{|l|l|} 
CH020104 & in Keyword list \\
\end{tabular} & [if gname] \\
\hline & & \begin{tabular}{|l|l|} 
CH020105 & missingValue \\
\end{tabular} & [if no name] \\
\hline $\mathrm{PAO} 2$ & GRID D & ef & \\
\hline & CA0201 & definition in I SQUARE, RE & CTANGULAR, HEXAGONAL, TRIANGULAR \\
\hline & & \begin{tabular}{|l|l|l|} 
CH020101 & SQUARE \\
\end{tabular} & [property G Square] \\
\hline B & & \begin{tabular}{|l|l|} 
CH020102 & RECTANGULAR \\
\end{tabular} & [property G Rectangular] \\
\hline & & \begin{tabular}{|l|l|} 
CH020103 & HEXAGONAL \\
\end{tabular} & [property G Hexagonal] \\
\hline & & \begin{tabular}{|l|l|} 
CH020104 TRIANGULAR \\
\end{tabular} & [property G Triangular] \\
\hline & CA0202 & other nominal value & \\
\hline & & CH020201 INVALID VALUE & [ERROR] \\
\hline B & & \begin{tabular}{|l|l|} 
CH020202 & missingValue \\
\end{tabular} & [property G Missing] \\
\hline PA03 & Grid E & lem Def & \\
\hline & CA0301 & definition in \{ STEP, PSTE & P, ANGLE, PX $\}$ \\
\hline & & \begin{tabular}{|l|l|} 
CH030101 & STEP \\
\end{tabular} & $\begin{array}{l}{[\text { if G_Square or G_Hexagonal, }} \\
\text { property step] }\end{array}$ \\
\hline B & & \begin{tabular}{|l|l|} 
CH030102 & PSTEP \\
\end{tabular} & $\begin{array}{l}\text { [if G_Rectangular or G_Triangular, } \\
\text { property pstep] }\end{array}$ \\
\hline & & \begin{tabular}{|l|l|l} 
CH030103 ANGLE \\
\end{tabular} & [if G Triangular, property angle] \\
\hline & & \begin{tabular}{|l|l|}
$\mathrm{CH} 030104$ & $\mathrm{PX}$ \\
\end{tabular} & [if G Triangular, property lateral] \\
\hline & CA0301 & Other value & \\
\hline & & CH030201 INVALID VALUE & [ERROR] \\
\hline B & & CH030202 missingValue & [if G Missing] \\
\hline PA0 4 & Grid E & Iem Val1 & \\
\hline & $\mathrm{CA0401}$ & valid value & \\
\hline & & $\mathrm{CH040101}=0$ & [if step or pstep or angle] \\
\hline B & & CH040102 $>0$ & [if step or pstep or angle] \\
\hline & & \begin{tabular}{|l|l|l} 
CH040103 & missingValue \\
\end{tabular} & [if G Missing or lateral] \\
\hline & CA0 402 & invalid value & \\
\hline & & CHO40201 $<0$ & [ERROR] [if step or pstep or angle] \\
\hline & & \begin{tabular}{|l|l|l}
$\mathrm{CH} 040202$ & non-number \\
\end{tabular} & [ERROR] [if step or pstep or angle] \\
\hline
\end{tabular}




\begin{tabular}{|c|c|c|}
\hline \multirow{4}{*}{\begin{tabular}{|l|}
$\mathrm{BA05}$ \\
$\mathrm{PA}$ \\
\end{tabular}} & \begin{tabular}{|l|l|} 
CH040203 & ErrorMissing \\
\end{tabular} & [ERROR] [if step or pstep or angle] \\
\hline & Grid Elem Val2 & \\
\hline & \begin{tabular}{l|l} 
CA0501 valid value \\
\end{tabular} & \\
\hline & $\mid$\begin{tabular}{l|l} 
CH050101 & $=0$ \\
\end{tabular} & [if pstep or angle] \\
\hline \multirow[t]{3}{*}{ B } & \begin{tabular}{|c|c|c|}
$\mathrm{CH} 050102$ & $>0$ \\
\end{tabular} & [if pstep or angle] \\
\hline & $\begin{array}{l}\text { CH050103 missingValue } \\
\text { miss }\end{array}$ & [if step or lateral] \\
\hline & \begin{tabular}{|l|l|l|} 
CA0502 & invalid value \\
\end{tabular} & \\
\hline \multirow[t]{3}{*}{ B } & \begin{tabular}{|l|l|l|} 
& CH050201 & $<0$ \\
\end{tabular} & [ERROR] [if pstep or angle] \\
\hline & \begin{tabular}{|l|l|}
$\mathrm{CH} 050202$ & non-number \\
\end{tabular} & [ERROR] [if pstep or angle] \\
\hline & \begin{tabular}{|l|l|} 
CH050203 & ErrorMissing \\
\end{tabular} & [ERROR] [if G Square or lateral] \\
\hline \multirow{5}{*}{ PA06 } & Grid Elem Triang Vall & \\
\hline & CA0601|number & \\
\hline & $\mid$ CH060101 $\mid=0$ & [if angle or literal] \\
\hline & \begin{tabular}{l|l|l}
$\mathrm{CH} 060102$ & $>0$ \\
\end{tabular} & [if angle or literal] \\
\hline & $\begin{array}{l}\mathrm{CH} 060103 \\
\end{array}$ & [if angle or literal] \\
\hline \multirow[t]{3}{*}{ B } & $\begin{array}{l}\text { CH060104 } \\
\text { missingValue }\end{array}$ & [if not (angle or literal)] \\
\hline & \begin{tabular}{|l|l|} 
CA0602 & non number \\
\end{tabular} & \\
\hline & \begin{tabular}{|l|l|l|} 
& CH060201 & non-number \\
\end{tabular} & [ERROR] [if angle or literal] \\
\hline $\mathrm{B}$ & \begin{tabular}{|l|l|} 
CH060202 & ErrorMissing \\
\end{tabular} & [ERROR] [if angle or literal] \\
\hline \multirow[t]{3}{*}{ PA07 } & Grid Elem Triang Val2 & \\
\hline & CA0701|number & \\
\hline & \begin{tabular}{|l|l|l} 
& $\mathrm{CH} 070101$ & $=0$
\end{tabular} & [if literal] \\
\hline \multirow[t]{5}{*}{$\mathrm{B}$} & $\mathrm{CHO70102}>0$ & [if literal] \\
\hline & \begin{tabular}{|l|l|}
$\mathrm{CH} 070103$ & $<0$ \\
\end{tabular} & [if literal] \\
\hline & \begin{tabular}{|l|l|} 
CH070104 missingValue \\
\end{tabular} & [if not literal] \\
\hline & \begin{tabular}{|l|l|} 
CA0702 & non number \\
\end{tabular} & \\
\hline & \begin{tabular}{|l|l|l|} 
& CH070201 & non-number \\
\end{tabular} & [ERROR] [if literal] \\
\hline $\mathrm{B}$ & \begin{tabular}{|l|l|} 
CH070202 & ErrorMissing \\
\end{tabular} & [ERROR] [if literal] \\
\hline \multirow[t]{3}{*}{ PA08 } & Grid Elem Triang Val3 & \\
\hline & CA0801|number & \\
\hline & $|\mathrm{CH} 080101|=0$ & [if literal] \\
\hline \multirow[t]{4}{*}{$\mathrm{B}$} & CH080102 $>0$ & [if literal] \\
\hline & \begin{tabular}{|l|l|}
$\mathrm{CH} 080103$ & $<0$ \\
\end{tabular} & [if literal] \\
\hline & \begin{tabular}{|l|l|l|} 
& CH080104 & missingValue \\
\end{tabular} & [if not literal] \\
\hline & \begin{tabular}{|l|l|} 
CA0802 & non number \\
\end{tabular} & \\
\hline \multirow[t]{2}{*}{ B } & \begin{tabular}{|l|l|l|} 
& CH080201 & non-number \\
\end{tabular} & [ERROR] [if literal] \\
\hline & \begin{tabular}{|l|l|} 
CH080202 & ErrorMissing \\
\end{tabular} & [ERROR] [if literal] \\
\hline \multirow[t]{3}{*}{ PA09 } & Grid Elem Triang Val3 & \\
\hline & CA0901 number & \\
\hline & CH090101 $=0$ & [if literal] \\
\hline \multirow[t]{5}{*}{ B } & $\mathrm{CH} 090102>0$ & [if literal] \\
\hline & CH090103 $<0$ & [if literal] \\
\hline & \begin{tabular}{l|l|l|l} 
& CH090104 & missingValue \\
\end{tabular} & [if not literal] \\
\hline & $\begin{array}{ll}\text { CA0902 } & \text { non number } \\
\end{array}$ & \\
\hline & CH090201 non-number & [ERROR] [if literal] \\
\hline $\mathrm{B}$ & \begin{tabular}{|l|l|l|} 
& CH090202 & ErrorMissing \\
\end{tabular} & [ERROR] [if literal] \\
\hline \multirow{3}{*}{ PA10 } & Grid_Elem Unit & \\
\hline & CA1001 Definition in $1 \mathrm{dm}, \mathrm{cm}$, & $\mathrm{m} \mathrm{\}}$ \\
\hline & \begin{tabular}{|l|l|l|} 
& $\mathrm{CH} 100101$ & $\mathrm{dm}$ \\
\end{tabular} & [if step or pstep] \\
\hline \multirow[t]{5}{*}{$\mathrm{B}$} & \begin{tabular}{|l|l|}
$\mathrm{CH} 100102$ & $\mathrm{~cm}$ \\
\end{tabular} & [if step or pstep] \\
\hline & $\mathrm{CH} 100103 \mathrm{~mm}$ & [if step or pstep] \\
\hline & \begin{tabular}{l|l|l} 
& $\mathrm{CH} 100104$ & $\mathrm{~m}$ \\
\end{tabular} & [if step or pstep] \\
\hline & \begin{tabular}{|l|l|l|l} 
CA1002 & Other value \\
\end{tabular} & \\
\hline & \begin{tabular}{|l|l|l|l|l} 
& CH100201 & INVALID VALUE
\end{tabular} & [if step or pstep] \\
\hline
\end{tabular}




\begin{tabular}{|c|c|c|c|}
\hline \multirow{4}{*}{\begin{tabular}{|l|l|} 
PA11 \\
\end{tabular}} & & CH100202 MissingValue & \\
\hline & Grid_E & lem Angle_Unit & \\
\hline & CA1101 & Definition in $\{\mathrm{deg}, \mathrm{rad}\}$ & \\
\hline & & \begin{tabular}{|l|l|} 
CH110101 deg \\
\end{tabular} & [if angle] \\
\hline \multirow[t]{3}{*}{$\mathrm{B}$} & & \begin{tabular}{|l|l|l|l} 
CH110102 & $\mathrm{rad}$ \\
\end{tabular} & [if angle] \\
\hline & CA1102 & Other value & \\
\hline & & \begin{tabular}{|l|l|} 
CH110201 INVALID VALUE \\
\end{tabular} & [if angle] \\
\hline$B$ & & CH110202 missingValue & \\
\hline \multirow[t]{2}{*}{ PA12 } & Elemen & tt Name & \\
\hline & CA1201 & Definition in \{GEOMETRY\} & \\
\hline \multirow[t]{3}{*}{ B } & & \begin{tabular}{|l|l|} 
CH120101 & GEOMETRY \\
\end{tabular} & [property geometry] \\
\hline & CA1202 & length of string & \\
\hline & & CH120201 length $=0$ & $\begin{array}{l}\text { [if not geometry, property } \\
\text { missing Name] }\end{array}$ \\
\hline \multirow[t]{3}{*}{$B$} & & \begin{tabular}{|l|l|} 
CH120202 & length [1..29] \\
\end{tabular} & [if not geometry, property E Name] \\
\hline & & \begin{tabular}{|l|l|} 
CH120203 & length $>=30$ \\
\end{tabular} & $\begin{array}{l}\text { [if not geometry, property } \\
\text { long Name] }\end{array}$ \\
\hline & CA1203 & context of name & \\
\hline \multirow[t]{4}{*}{ B } & & \begin{tabular}{|l|l|l|l} 
CH120301 & start with Alpha \\
\end{tabular} & [if E Name] \\
\hline & & \begin{tabular}{|l|l|} 
CH120302 & start with Number \\
\end{tabular} & [if E Name] \\
\hline & & \begin{tabular}{|l|l|} 
CH120303 & $\begin{array}{l}\text { start_with_other_ } \\
\text { Char }\end{array}$ \\
\end{tabular} & [if E_Name. \\
\hline & & \begin{tabular}{|l|l|} 
CH120304 & in Keyword list \\
\end{tabular} & [if E Name \\
\hline \multirow[t]{2}{*}{ PA13 } & Elem $\mathrm{G}$ & Seometry Def & \\
\hline & CA1301 & Definition in \{CIRCULAR, R] & ECTANGULAR \} \\
\hline \multirow[t]{3}{*}{ B } & & \begin{tabular}{|l|l|} 
CH130101 & CIRCULAR \\
\end{tabular} & [if geometry, property E Circular] \\
\hline & & \begin{tabular}{|l|l|} 
CH130102 & RECTANGULAR \\
\end{tabular} & $\begin{array}{l}\text { [if geometry, property } \\
\text { E Rectangular] }\end{array}$ \\
\hline & $\overline{\mathrm{CA} 1302}$ & $\begin{array}{l}\text { Other } \\
\text { value }\end{array}$ & \\
\hline \multirow[t]{2}{*}{$B$} & & \begin{tabular}{|l|l|l|} 
CH130201 & INVALID VALUE \\
\end{tabular} & [if geometry, property wrong E Def] \\
\hline & & CH130202 missingValue & [if missing Name] \\
\hline \multirow[t]{3}{*}{ PA14 } & Elem G & Seometry Vall & \\
\hline & CA1401 & unsigned real number & \\
\hline & & \begin{tabular}{|l|l|} 
CH140101 & $=0$ \\
\end{tabular} & [if E Circular or E Rectangular] \\
\hline \multirow[t]{2}{*}{$\mathrm{B}$} & & \begin{tabular}{|l|l|l|l|l|l|}
$\mathrm{CH} 140102$ & $>0$ \\
\end{tabular} & [if E Circular or E Rectangular] \\
\hline & CA1402 & wrong value & \\
\hline \multirow[t]{3}{*}{ B } & & $\mid \mathrm{CH} 140201<0$ & $\begin{array}{l}\text { [ERROR] [if E_Circular or } \\
\text { E Rectangular] }\end{array}$ \\
\hline & & CH140202 non-number & $\begin{array}{l}\text { [ERROR] [if E_Circular or } \\
\text { E Rectangular] }\end{array}$ \\
\hline & & \begin{tabular}{|l|l|l} 
CH140203 & missingValue \\
\end{tabular} & [if wrong_E_Def or not geometry] \\
\hline \multirow[t]{3}{*}{ PA15 } & Elem_G & Geometry Val2 & \\
\hline & CA1501 & unsigned real number & \\
\hline & & \begin{tabular}{|l|l|l|l|l|} 
CH150101 & $=0$ \\
\end{tabular} & [if E Rectangular] \\
\hline \multirow[t]{4}{*}{$B$} & & \begin{tabular}{ll|l}
$\mathrm{CH} 150102$ & $>0$ \\
\end{tabular} & [if E Rectangular] \\
\hline & CA1502 & wrong value & \\
\hline & & $\mid \mathrm{CH} 150201<0$ & [ERROR] [if E Rectangular] \\
\hline & & \begin{tabular}{|l|l|} 
CH150202 & non-number \\
\end{tabular} & [ERROR] [if E Rectangular] \\
\hline \multirow[t]{2}{*}{ B } & & \begin{tabular}{|l|l|} 
CH150203 & ErrorMissing \\
\end{tabular} & [ERROR] [if E Rectangular] \\
\hline & & CH150204 missingValue & [if not E Rectangular] \\
\hline \multirow{4}{*}{ PA16 } & Elem G & seometry Unit & \\
\hline & CA1601 & Definition in $\{\mathrm{dm}, \mathrm{cm}, \mathrm{mm}, \mathrm{m}\}$ & \\
\hline & & \begin{tabular}{|l|l|} 
CH160101 & $\mathrm{dm}$ \\
\end{tabular} & [if E Circular or E Rectangular] \\
\hline & & \begin{tabular}{|l|l|l|l}
$\mathrm{CH} 160102$ & $\mathrm{~cm}$ \\
\end{tabular} & [if E Circular or E Rectangular] \\
\hline
\end{tabular}




\begin{tabular}{|c|c|c|c|c|}
\hline B & & $\frac{\mathrm{CH} 160103}{\mathrm{CH} 160104}$ & $\mathrm{~mm}$ & [if E Circular or E Rectangular] \\
\hline & & & & \\
\hline & CA1602 & Other va & lue & \\
\hline & & $\mathrm{CH} 160201$ & INVALID_VALUE & $\begin{array}{l}\text { [ERROR] [if E_Circular or } \\
\text { E Rectangular] }\end{array}$ \\
\hline $\mathrm{B}$ & & CH160202 & missingValue & \\
\hline PA17 & Elem $\mathrm{B}$ & olarizati & on Spec & \\
\hline & CA1701 & Definitis & on in $\{$ LINEAR, & RCULAR\} \\
\hline B & & CH170101 & LINEAR & [if geometry, property E P linear \\
\hline & & CH170102 & CIRCULAR & $\begin{array}{l}\text { [if geometry, property } \\
\text { E P circular] }\end{array}$ \\
\hline & CA1702 & $\begin{array}{l}\text { Other } \\
\text { value }\end{array}$ & & \\
\hline & & CH170201 & INVALID VALUE & [ERROR] \\
\hline $\mathrm{B}$ & & CH170202 & missingValue & \\
\hline PA18 & Elem $\mathrm{F}$ & olar Def & & \\
\hline & CA1801 & Definiti & on in [ORIENTAT & LH, $\mathrm{RH}\}$ \\
\hline B & & $\mathrm{CH} 180101$ & ORIENTATION & $\begin{array}{l}{[\text { if E P linear, property }} \\
\text { E P orientation] }\end{array}$ \\
\hline & & CH180102 & $\mathrm{LH}$ & {$\left[\begin{array}{llll}\text { if } & E & \text { P } & \text { circular }\end{array}\right.$} \\
\hline & & $\mathrm{CH} 180103$ & $\mathrm{RH}$ & [if \\
\hline & CA1802 & Other va. & Iue & \\
\hline B & & CH180201 & INVALID_VALUE & $\begin{array}{l}\text { [ERROR] [if E P_linear or } \\
\text { E P circular] }\end{array}$ \\
\hline & & CH180202 & missingValue & \\
\hline PA19 & Elem_B & Olar Line & Pol Val & \\
\hline & CA1901 & Definiti & on in $\{X, Y\}$ & \\
\hline $\mathrm{B}$ & & CH190101 & $\mathrm{X}$ & [if E P orientation] \\
\hline & & CH190102 & $\mathrm{Y}$ & [if E P orientation] \\
\hline & CA1902 & real numb & ber & \\
\hline & & CH190201 & $=0$ & [if E P orientation] \\
\hline $\bar{B}$ & & CH190202 & $>0$ & [if E P orientation] \\
\hline & & CH190203 & $<0$ & {$\left[\begin{array}{llll}\text { if } & E & P & \text { linear }\end{array}\right]$} \\
\hline & CA1903 & Other va. & lue & \\
\hline & & CH190301 & INVALID VALUE & [ERROR] [if $\mathrm{E}$ P orientation] \\
\hline & & CH190302 & errorMissing & [ERROR] [if E $\mathrm{P}$ orientation] \\
\hline B & & CH190302 & missingValue & \\
\hline PA20 & $\sqrt{E l e m ~}$ & olar Line & Pol Unit & \\
\hline & CA2001 & Definiti & on in deg, rad & \\
\hline $\mathrm{B}$ & & CH200101 & $\operatorname{deg}$ & [if $\mathrm{E}$ P orientation] \\
\hline & & $\mathrm{CH} 200102$ & rad & [if E P orientation] \\
\hline & $\overline{C A 2002}$ & null valu & & \\
\hline $\mathrm{B}$ & & $\mathrm{CH} 200201$ & INVALID VALUE & [ERROR] [if $\mathrm{E}$ P orientation] \\
\hline & & $\mathrm{CH} 200202$ & missingvalue & \\
\hline PA21 & Elem $\mathrm{B}$ & ort Numbe & & \\
\hline & CA2101 & Natural & number & \\
\hline & & CH210101 & $=0$ & [if geometry, property no Port] \\
\hline $\mathrm{B}$ & & $\mathrm{CH} 210102$ & {$[1,998]$} & $\begin{array}{l}\text { [if geometry, property } \\
\text { medium size Port] }\end{array}$ \\
\hline & & $\mathrm{CH} 210103$ & $=999$ & $\begin{array}{l}\text { [if geometry, property } \\
\text { large Size Port] }\end{array}$ \\
\hline & CA2102 & other nur & mber & \\
\hline & & $\mathrm{CH} 210201$ & $>999$ & if geometry] \\
\hline $\mathrm{B}$ & & CH210202 & $<0$ & if geometry] \\
\hline
\end{tabular}




\begin{tabular}{|c|c|c|c|}
\hline & & \begin{tabular}{|l|l|} 
CH210203 & real number \\
\end{tabular} & [ERROR] [ if geometry] \\
\hline & CA2103 & other value & \\
\hline & & \begin{tabular}{|l|l|l|} 
CH210301 & INVALID_VALUE \\
\end{tabular} & [ERROR] [ if geometry] \\
\hline B & & \begin{tabular}{|l|l|l|l|l} 
CH21030ingValue \\
\end{tabular} & \\
\hline \multirow[t]{4}{*}{ PA22 } & Elem $P$ & ort set ENum & \\
\hline & CA2201 & number of the element & \\
\hline & & CH220101 $=0$ & $\begin{array}{l}\text { [if no Port, property } \\
\text { missingPortDef] }\end{array}$ \\
\hline & & $\mathrm{CH} 220102$ & $\begin{array}{l}\text { [if medium_Size_Port, property } \\
\text { onePortDef] }\end{array}$ \\
\hline B & & CH220103 & $\begin{array}{l}\text { [if medium_Size Port or } \\
\text { large Size Port] }\end{array}$ \\
\hline \multirow[t]{4}{*}{ PA23 } & Elem $\mathrm{PC}$ & ort Orient Val & \\
\hline & CA2301 & content of the set & \\
\hline & & $\mathrm{CH} 230101 \mathrm{X}$ & [if not missingPortDef] \\
\hline & & \begin{tabular}{|l|l|l}
$\mathrm{CH} 230102$ & $\mathrm{Y}$ \\
\end{tabular} & [if not missingPortDef] \\
\hline \multirow[t]{7}{*}{$B$} & & CH230103 $>0$ & [if not missingPortDef] \\
\hline & & \begin{tabular}{|l|l|l|l|l}
$\mathrm{CH} 230104$ & $=0$ \\
\end{tabular} & [if not missingPortDef] \\
\hline & & $\mathrm{CH} 230105$ & [if not missingPortDef] \\
\hline & & combination & $\begin{array}{l}\text { [if not (missingPortDef or } \\
\text { onePortDef)] }\end{array}$ \\
\hline & & \begin{tabular}{|l|l|}
$\mathrm{CH} 230107$ & ContainInvalid \\
\end{tabular} & $\begin{array}{l}\text { [if not (missingPortDef or } \\
\text { onePortDef)] }\end{array}$ \\
\hline & & \begin{tabular}{|l|l|} 
CH230108 & allinvalid \\
\end{tabular} & [if not missingPortDef] \\
\hline & & CH230109 missingValue & \\
\hline \multirow[t]{4}{*}{ PA24 } & Elem $\mathrm{P}$ & ort orient UNIT & \\
\hline & CA2401 & content of the set & \\
\hline & & \begin{tabular}{ll|l}
$\mathrm{CH} 240101$ & deg \\
\end{tabular} & [if not missingPortDef] \\
\hline & & \begin{tabular}{|l|l|}
$\mathrm{CH} 240102$ & $\mathrm{rad}$ \\
\end{tabular} & [if not missingPortDef] \\
\hline \multirow[t]{4}{*}{ B } & & CH240103 combination & $\begin{array}{l}\text { [if not (missingPortDef or } \\
\text { onePortDef)] }\end{array}$ \\
\hline & & \begin{tabular}{|l|l} 
CH240104 mixedValue \\
\end{tabular} & $\begin{array}{l}\text { [if not (missingPortDef or } \\
\text { onePortDef)] }\end{array}$ \\
\hline & & CH240105 allinvalid & [if not missingPortDef] \\
\hline & & \begin{tabular}{|l|l|l} 
CH240106 & missingValue \\
\end{tabular} & \\
\hline \multirow[t]{5}{*}{ PA25 } & Elem $P C$ & ort Amp Val & \\
\hline & CA2501 & content of the set & \\
\hline & & $\mathrm{CH} 250101>0$ & [if not missingPortDef] \\
\hline & & $\mathrm{CH} 250102=0$ & [if not missingPortDef] \\
\hline & & CH250103 $<0$ & [if not missingPortDef] \\
\hline \multirow[t]{4}{*}{ B } & & combination & $\begin{array}{l}\text { [if not (missingPortDef or } \\
\text { onePortDef)] }\end{array}$ \\
\hline & & CH250105 ContainInvalid & $\begin{array}{l}\text { [if not (missingPortDef or } \\
\text { onePortDef)] }\end{array}$ \\
\hline & & \begin{tabular}{|l|l|} 
CH250106 & allinvalid \\
\end{tabular} & [if not missingPortDef] \\
\hline & & \begin{tabular}{|l|l|}
$\mathrm{CH} 250107$ & missingValue \\
\end{tabular} & \\
\hline \multirow[t]{8}{*}{ PA26 } & Elem Pc & ort Amp Unit & \\
\hline & CA2 601 & content of the set & \\
\hline & & \begin{tabular}{|l|l|}
$\mathrm{CH} 260101$ & $\mathrm{dm}$ \\
\end{tabular} & [if not missingPortDef] \\
\hline & & \begin{tabular}{|l|l|}
$\mathrm{CH} 260102$ & $\mathrm{~mm}$ \\
\end{tabular} & [if not missingPortDef] \\
\hline & & \begin{tabular}{|l|l|}
$\mathrm{CH} 260103$ & $\mathrm{~cm}$ \\
\end{tabular} & [if not missingPortDef] \\
\hline & & \begin{tabular}{|l|l|l}
$\mathrm{CH} 260104 \mathrm{~m}$ \\
\end{tabular} & [if not missingPortDef] \\
\hline & & \begin{tabular}{|l|l|}
$\mathrm{CH} 260105$ & $\mathrm{~dB}$ \\
\end{tabular} & [if not missingPortDef] \\
\hline & & \begin{tabular}{|l|l|} 
CH260106 & POWER \\
\end{tabular} & [if not missingPortDef] \\
\hline B & & \begin{tabular}{|l|l|} 
CH260107 LINEAR \\
\end{tabular} & [if not missingPortDef] \\
\hline
\end{tabular}




\begin{tabular}{|c|c|c|c|c|}
\hline & $\mathrm{CH} 260108$ & combination & $\begin{array}{l}\text { [if not (missingPortDef } 0 \\
\text { onePortDef)] }\end{array}$ & \\
\hline & $\mathrm{CH} 260109$ & ContainInvalid & $\begin{array}{l}\text { [if not (missingPortDef } \\
\text { onePortDef)] }\end{array}$ & \\
\hline & $\mathrm{CH} 260110$ & allinvalid & [if not missingPortDef] & \\
\hline & $\mathrm{CH} 260111$ & missingValue & & \\
\hline PA27 & Elem Port Shdef & Val & & \\
\hline & CA2701 content & of the set & & \\
\hline & \begin{tabular}{|l|l|} 
& $\mathrm{CH} 270101$ \\
\end{tabular} & $>0$ & [if not missingPortDef] & \\
\hline & $\mathrm{CH} 270102$ & $=0$ & [if not missingPortDef] & \\
\hline & $\mathrm{CH} 270103$ & $<0$ & [if not missingPortDef] & \\
\hline B & $\mathrm{CH} 270104$ & combination & $\begin{array}{l}\text { [if not (missingPortDef } \circ \\
\text { onePortDef)] }\end{array}$ & or \\
\hline & $\mathrm{CH} 270105$ & ContainInvalid & $\begin{array}{l}\text { [if not (missingPortDef o } \\
\text { onePortDef)] }\end{array}$ & or \\
\hline & $\mathrm{CH} 270106$ & allInvalid & [if not missingPortDef] & \\
\hline & $\mathrm{CH} 270107$ & MissingValue & & \\
\hline PA28 & Elem Port Shdef & Unit & & \\
\hline & \begin{tabular}{|l|l|} 
CA280ntent \\
\end{tabular} & of the set & & \\
\hline & \begin{tabular}{|l|l|}
$\mathrm{CH} 280101$ \\
\end{tabular} & deg & [if not missingPortDef] & \\
\hline $\mathrm{B}$ & $\mathrm{CH} 280102$ & rad & [if not missingPortDef] & \\
\hline & $\mathrm{CH} 280103$ & combination & $\begin{array}{l}\text { [if not (missingPortDef o } \\
\text { onePortDef)] }\end{array}$ & or \\
\hline & $\mathrm{CH} 280104$ & ContainInvalid & $\begin{array}{l}\text { [if not (missingPortDef o } \\
\text { onePortDef)] }\end{array}$ & or \\
\hline & CH280105 & allinvalid & [if not missingPortDef] & \\
\hline & $\mathrm{CH} 280106$ & missingValue & & \\
\hline PA29 & Elem Port Scdef & Val & & \\
\hline & \begin{tabular}{|l|l|} 
CA2901 content \\
\end{tabular} & of the set & & \\
\hline & \begin{tabular}{|l|l|} 
& $\mathrm{CH} 290101$ \\
\end{tabular} & $>0$ & [if not missingPortDef] & \\
\hline & $\mathrm{CH} 290102$ & $=0$ & [if not missingPortDef] & \\
\hline & $\mathrm{CH} 290103$ & $<0$ & [if not missingPortDef] & \\
\hline$B$ & $\mathrm{CH} 290104$ & combination & $\begin{array}{l}{[\text { if not (missingPortDef o }} \\
\text { onePortDef)] }\end{array}$ & or \\
\hline & CH290105 & ContainInvalid & $\begin{array}{l}\text { [if not (missingPortDef o } \\
\text { onePortDef)] }\end{array}$ & or \\
\hline & CH290106 & allinvalid & [if not missingPortDef] & \\
\hline & \begin{tabular}{l|l}
$\mathrm{CH} 290107$ \\
\end{tabular} & missingvalue & & \\
\hline PA30 & Elem Port Scdef & Enit & & \\
\hline & CA3001 content & of the set & & \\
\hline & \begin{tabular}{|l|l|} 
& $\mathrm{CH} 300101$ \\
\end{tabular} & deg & [if not missingPortDef] & \\
\hline B & $\mathrm{CH} 300102$ & $\mathrm{rad}$ & [if not missingPortDef] & \\
\hline & CH300103 & combination & $\begin{array}{l}{[\text { if not (missingPortDef o }} \\
\text { onePortDef)] }\end{array}$ & or \\
\hline & CH300104 & ContainInvalid & $\begin{array}{l}\text { [if not (missingPortDef } \\
\text { onePortDef)] }\end{array}$ & or \\
\hline & CH300105 & allinvalid & [if not missingPortDef] & \\
\hline & Elem Port Padef & E Val & & \\
\hline & \begin{tabular}{|l|l|} 
CA3101 & content \\
\end{tabular} & of the set & & \\
\hline & CH310101 & $>0$ & [if not missingPortDef] & \\
\hline & CH310102 & $=0$ & [if not missingPortDef] & \\
\hline & $\mathrm{CH} 310103$ & $<0$ & [if not missingPortDef] & \\
\hline & $\mathrm{CH} 310104$ & combination & $\begin{array}{l}\text { [if not (missingPortDef o } \\
\text { onePortDef)] }\end{array}$ & or \\
\hline & $\mathrm{CH} 310105$ & ContainInvalid & [if not (missingPortDef & or \\
\hline
\end{tabular}




\begin{tabular}{|c|c|c|c|c|}
\hline & & & & onePortDef)] \\
\hline & & CH310106 & allinvalid & [if not missingPortDef] \\
\hline & & CH310107 & missingValue & \\
\hline \multirow{3}{*}{ PA32 } & Elem P & ort Padef & Unit & \\
\hline & CA3201 & content 0 & of the set & \\
\hline & & CH320101 & deg & [if not missingPortDef] \\
\hline \multirow[t]{4}{*}{ B } & & CH320102 & rad & [if not missingPortDef] \\
\hline & & $\mathrm{CH} 320103$ & combination & $\begin{array}{l}\text { [if not (missingPortDef or } \\
\text { onePortDef)] }\end{array}$ \\
\hline & & $\mathrm{CH} 320104$ & ContainInvalid & $\begin{array}{l}\text { [if not (missingPortDef or } \\
\text { onePortDef)] }\end{array}$ \\
\hline & & CH320105 & allinvalid & [if not missingPortDef] \\
\hline \multirow[t]{2}{*}{ PA33 } & Grgeo & Add Def & & \\
\hline & CA3301 & Definitic & on in HEXAGON, & OCK, NODE, POLYGON\} \\
\hline \multirow[t]{7}{*}{ B } & & $\mathrm{CH} 330101$ & NODE & [property node] \\
\hline & & $\mathrm{CH} 330102$ & HEXAGON & [property hexagon] \\
\hline & & CH330103 & BLOCK & [property block] \\
\hline & & $\mathrm{CH} 330104$ & POLYGON & [property polygon] \\
\hline & CA3302 & Other val & lue & \\
\hline & & $\mathrm{CH} 330201$ & INVALID VALUE & [ERROR] \\
\hline & & CH330202 & errorMissing & [ERROR] \\
\hline \multirow[t]{4}{*}{ PA34 } & Grgeo & Add Int $V$ & lal1 & \\
\hline & CA3401 & integer & & \\
\hline & & CH340101 & $<0$ & $\begin{array}{l}\text { [if node or hexagon or block or } \\
\text { polygon] }\end{array}$ \\
\hline & & CH340102 & $=0$ & $\begin{array}{l}\text { [if node or hexagon or block or } \\
\text { polygon] }\end{array}$ \\
\hline \multirow[t]{4}{*}{ B } & & $\mathrm{CH} 340103$ & $>0$ & \begin{tabular}{|l} 
[if node or hexagon or block or \\
polygon]
\end{tabular} \\
\hline & CA3402 & Other val & lue & \\
\hline & & CH340201 & real number & $\begin{array}{l}\text { [ERROR] [if node or hexagon or } \\
\text { block or polygon] }\end{array}$ \\
\hline & & $\mathrm{CH} 340202$ & non-number & $\begin{array}{l}\text { [ERROR] [if node or hexagon or } \\
\text { block or polygon] }\end{array}$ \\
\hline $\mathrm{B}$ & & $\mathrm{CH} 340203$ & errorMissing & $\begin{array}{l}\text { [ERROR] [if node or hexagon or } \\
\text { block or polygon] }\end{array}$ \\
\hline \multirow[t]{4}{*}{ PA35 } & Grgeo & Add Int $V$ & Tal2 & \\
\hline & CA3501 & integer & & \\
\hline & & CH350101 & $<0$ & $\begin{array}{l}\text { [if node or hexagon or block or } \\
\text { polygon] }\end{array}$ \\
\hline & & CH350102 & $=0$ & $\begin{array}{l}\text { [if node or hexagon or block or } \\
\text { polygon] }\end{array}$ \\
\hline \multirow[t]{2}{*}{$B$} & & СH350103 & $>0$ & $\begin{array}{l}\text { [if node or hexagon or block or } \\
\text { polygon] }\end{array}$ \\
\hline & $\mathrm{CA} 3502$ & Other val & lue & \\
\hline \multirow[t]{3}{*}{ B } & & CH350201 & real number & $\begin{array}{l}\text { [ERROR] [if node or hexagon or } \\
\text { block or polygon] }\end{array}$ \\
\hline & & $\mathrm{CH} 350202$ & non-number & $\begin{array}{l}\text { [ERROR] [if node or hexagon or } \\
\text { block or polygon] }\end{array}$ \\
\hline & & CH350203 & errorMissing & $\begin{array}{l}\text { [ERROR] [if node or hexagon or } \\
\text { block or polygon] }\end{array}$ \\
\hline \multirow[t]{4}{*}{ PA36 } & Grgeo & Add Int V & $7 a 13$ & \\
\hline & CA 3601 & integer & & \\
\hline & & $\mathrm{CH} 360101$ & $<0$ & [if block or polygon] \\
\hline & & $\mathrm{CH} 360102$ & $=0$ & [if block or polygon] \\
\hline
\end{tabular}




\begin{tabular}{|c|c|c|c|}
\hline \multirow{3}{*}{$\mathrm{B}$} & & \begin{tabular}{|c|c|}
$\mathrm{CH} 360103$ & $>0$ \\
\end{tabular} & \multirow[t]{2}{*}{ [if block or polygon] } \\
\hline & CA3602 & Other value & \\
\hline & & \begin{tabular}{l|l|l} 
CH360201 & real number \\
\end{tabular} & [ERROR] [if block or polygon] \\
\hline & & \begin{tabular}{|l|l|} 
CH360202 & non-number \\
\end{tabular} & [ERROR] [if block or polygon] \\
\hline & & \begin{tabular}{|l|l|} 
CH360203 & errorMissing \\
\end{tabular} & [ERROR] [if block or polygon] \\
\hline$B$ & & \begin{tabular}{|l|l|} 
CH360204 & missingValue \\
\end{tabular} & [if not(block or polygon)] \\
\hline \multirow{4}{*}{ PA37 } & Grgeo & Add Int Val4 & \\
\hline & CA3701 & integer & \\
\hline & & \begin{tabular}{|l|l|l|l|l|} 
CH370101 & $<0$ \\
\end{tabular} & [if block or polygon] \\
\hline & & $\mathrm{CH} 370102=0$ & [if block or polygon] \\
\hline \multirow[t]{4}{*}{$\mathrm{B}$} & & CH370103 $>0$ & [if block or polygon] \\
\hline & CA3702 & Other value & \\
\hline & & \begin{tabular}{|l|l|} 
CH370201 & real number \\
\end{tabular} & [ERROR] [if block or polygon] \\
\hline & & \begin{tabular}{|l|l|} 
CH370202 & non-number \\
\end{tabular} & [ERROR] [if block or polygon] \\
\hline \multirow[t]{2}{*}{$\mathrm{B}$} & & \begin{tabular}{|l|l|} 
CH370203 & errorMissing \\
\end{tabular} & [ERROR] [if block or polygon] \\
\hline & & \begin{tabular}{|l|l} 
CH370204 missingValue \\
\end{tabular} & [if not(block or polygon)] \\
\hline \multirow[t]{4}{*}{ PA38 } & Grgeo & Add Int Val5 & \\
\hline & CA3801 & integer & \\
\hline & & $\mid$ CH380101 $<0$ & [if polygon] \\
\hline & & \begin{tabular}{|l|l}
$\mathrm{CH} 380102$ & $=0$ \\
\end{tabular} & [if polygon] \\
\hline \multirow[t]{5}{*}{$\mathrm{B}$} & & CH380103 $>0$ & [if polygon] \\
\hline & CA3802 & Other value & \\
\hline & & \begin{tabular}{|l|l|} 
CH380201 & real number \\
\end{tabular} & [ERROR] [if polygon] \\
\hline & & \begin{tabular}{|l|l|} 
CH380202 & non-number \\
\end{tabular} & [ERROR] [if polygon] \\
\hline & & \begin{tabular}{|l|l|} 
CH380203 & errorMissing \\
\end{tabular} & [ERROR] [if polygon] \\
\hline $\mathrm{B}$ & & \begin{tabular}{|l|l} 
CH380204 & missingValue \\
\end{tabular} & [if not (polygon) ] \\
\hline \multirow{4}{*}{ PA39 } & Grgeo & Add Int Val6 & \\
\hline & CA3901 & integer & \\
\hline & & \begin{tabular}{|l|l|l|l|} 
CH390101 & $<0$ \\
\end{tabular} & [if polygon] \\
\hline & & \begin{tabular}{|l|l} 
CH390102 $=0$ \\
\end{tabular} & [if polygon] \\
\hline \multirow[t]{3}{*}{$\mathrm{B}$} & & \begin{tabular}{|l|l|} 
CH390103 & $>0$
\end{tabular} & [if polygon] \\
\hline & CA3802 & Other value & \\
\hline & & \begin{tabular}{|l|l|} 
CH390201 & real number \\
\end{tabular} & [ERROR] [if polygon] \\
\hline \multirow[t]{3}{*}{$\mathrm{B}$} & & $\begin{array}{ll}\mathrm{CH} 390202 & \text { non-number } \\
\end{array}$ & [ERROR] [if polygon] \\
\hline & & CH390203 errorMissing & [ERROR] [if polygon] \\
\hline & & CH390204 missingValue & [if not(polygon)] \\
\hline \multirow[t]{4}{*}{ PA40 } & Grgeo & Add Real Vall & \\
\hline & CA4001 & real number & \\
\hline & & $\mid \mathrm{CH} 400101<0$ & [if node] \\
\hline & & \begin{tabular}{|l|l|}
$\mathrm{CH} 400102$ & $=0$
\end{tabular} & [if node] \\
\hline \multirow[t]{3}{*}{$\mathrm{B}$} & & \begin{tabular}{|c|c|}
$\mathrm{CH} 400103>0$ \\
\end{tabular} & [if node] \\
\hline & $\overline{\mathrm{CA} 4002}$ & $\begin{array}{l}\text { Other } \\
\text { value }\end{array}$ & \\
\hline & & \begin{tabular}{|l|l|}
$\mathrm{CH} 400201$ & non-number \\
\end{tabular} & [ERROR] [if node] \\
\hline $\bar{B}$ & & \begin{tabular}{|l|l}
$\mathrm{CH} 400202$ & missingValue \\
\end{tabular} & \\
\hline \multirow[t]{4}{*}{ PA41 } & Grgeo & Add Real Val2 & \\
\hline & CA4101 & real number & \\
\hline & & \begin{tabular}{|l|l|l|l|l|l|}
$\mathrm{CH} 410101$ & $<0$ \\
\end{tabular} & [if node] \\
\hline & & \begin{tabular}{|l|l}
$\mathrm{CH} 410102$ & $=0$ \\
\end{tabular} & [if node] \\
\hline \multirow[t]{3}{*}{$\mathrm{B}$} & & \begin{tabular}{|l|l|} 
CH4 10103 & $>0$ \\
\end{tabular} & [if node] \\
\hline & CA4102 & Other value & \\
\hline & & \begin{tabular}{|l|l}
$\mathrm{CH} 410201$ & non-number
\end{tabular} & [ERROR] [if node] \\
\hline $\mathrm{B}$ & & \begin{tabular}{|l|l} 
CH410202 missingValue \\
\end{tabular} & \\
\hline PA42 & & Add Real Val3 & \\
\hline
\end{tabular}




\begin{tabular}{|c|c|c|c|}
\hline & CA4201 & real number & \\
\hline & & \begin{tabular}{|l|l|l|l|}
$\mathrm{CH} 420101$ & $<0$ \\
\end{tabular} & [if node] \\
\hline & & \begin{tabular}{|l|l}
$\mathrm{CH} 420102$ & $=0$ \\
\end{tabular} & [if node] \\
\hline \multirow[t]{2}{*}{$\mathrm{B}$} & & $|\mathrm{CH} 420103|>0$ & [if node] \\
\hline & CA4202 & \begin{tabular}{|l|l|} 
Other & \\
value & \\
\end{tabular} & \\
\hline \multirow[t]{2}{*}{$\mathrm{B}$} & & \begin{tabular}{|l|l|} 
CH420201 & non-number \\
\end{tabular} & [ERROR] [if node] \\
\hline & & \begin{tabular}{|l|l|} 
CH4 20202 & missingValue \\
\end{tabular} & \\
\hline \multirow[t]{2}{*}{ PA43 } & Grgeo & Add Unit & \\
\hline & CA4301 & Definition in $\{$ deg, rad $\}$ & \\
\hline \multirow[t]{4}{*}{$\mathrm{B}$} & & \begin{tabular}{|l|l|l|}
$\mathrm{CH} 430101$ & deg \\
\end{tabular} & [if node] \\
\hline & & \begin{tabular}{|l|l|}
$\mathrm{CH} 430102$ & rad \\
\end{tabular} & [if node] \\
\hline & CA4302 & other value & \\
\hline & & \begin{tabular}{|l|l|} 
CH4 30201 & INVALID VALUE \\
\end{tabular} & [ERROR] [if node] \\
\hline $\mathrm{B}$ & & CH430202 missingValue & \\
\hline \multirow[t]{3}{*}{$\overline{\mathrm{PA} 44}$} & Grgeo & Additional def & \\
\hline & CA4401 & number of set element & \\
\hline & & \begin{tabular}{|l|l|}
$\mathrm{CH} 440101$ & $=0$ \\
\end{tabular} & [property missingAddset] \\
\hline \multirow[t]{3}{*}{ B } & & \begin{tabular}{|l|l|}
$\mathrm{CH} 440102$ & $=1$ \\
\end{tabular} & [property singleAddSet] \\
\hline & & \begin{tabular}{|l|l|}
$\mathrm{CH} 440103$ & $>1$ \\
\end{tabular} & [property moreAddElem] \\
\hline & CA4402 & content of the set & \\
\hline \multirow[t]{5}{*}{ B } & & \begin{tabular}{|l|l|} 
CH440201 & NODE \\
\end{tabular} & [if singleAddSet] \\
\hline & & \begin{tabular}{|l|l|l|}
$\mathrm{CH} 440202$ & BLOCK \\
\end{tabular} & [if singleAddSet] \\
\hline & & \begin{tabular}{|l|l|}
$\mathrm{CH} 440203$ & HEXAGON \\
\end{tabular} & [if singleAddSet] \\
\hline & & \begin{tabular}{|l|l|} 
CH440204 & combination \\
\end{tabular} & [if moreAddElem] \\
\hline & & \begin{tabular}{|l|l|} 
CH440205 & missingValue \\
\end{tabular} & [if missingAddSet] \\
\hline \multirow[t]{2}{*}{ PA45 } & Grgeo & Additional Int Vall & \\
\hline & СА4501 & content of the set & \\
\hline \multirow[t]{6}{*}{$\mathrm{B}$} & & \begin{tabular}{|l|l|l|l|l|l|}
$\mathrm{CH} 450101$ & $>0$ \\
\end{tabular} & [if not missingAddSet] \\
\hline & & \begin{tabular}{ll|l}
$\mathrm{CH} 450102$ & $<0$ \\
\end{tabular} & [if not missingAddset] \\
\hline & & \begin{tabular}{l|l}
$\mathrm{CH} 450103$ & $=0$ \\
\end{tabular} & [if not missingAddset] \\
\hline & & \begin{tabular}{|l|l|} 
CH450104 & combination \\
\end{tabular} & [if not missingAddSet] \\
\hline & & \begin{tabular}{l|l} 
CH450105 & Invalidvalue \\
\end{tabular} & [if not missingAddSet] \\
\hline & & \begin{tabular}{|l|l|} 
CH450106 & missingValue
\end{tabular} & \\
\hline \multirow[t]{2}{*}{ PA46 } & Grgeo & Additional Int Val2 & \\
\hline & CA4601 & content of the set & \\
\hline \multirow[t]{6}{*}{$B$} & & $|\mathrm{CH} 460101|>0$ & [if not missingAddSet] \\
\hline & & \begin{tabular}{|l|l|}
$\mathrm{CH} 460102$ & $<0$ \\
\end{tabular} & [if not missingAddset] \\
\hline & & \begin{tabular}{|l|l|}
$\mathrm{CH} 460103$ & $=0$ \\
\end{tabular} & [if not missingAddSet] \\
\hline & & \begin{tabular}{|l|l|} 
CH4 60104 & Combination \\
\end{tabular} & [if not missingAddset] \\
\hline & & \begin{tabular}{|l|l|} 
CH 460105 & InvalidValue \\
\end{tabular} & [if not missingAddSet] \\
\hline & & \begin{tabular}{|l|l} 
CH4 60106 & missingValue \\
\end{tabular} & \\
\hline \multirow{2}{*}{ PA47 } & Grgeo & Additional Int Val3 & \\
\hline & CA4701 & content of the set & \\
\hline \multirow[t]{6}{*}{ B } & & \begin{tabular}{|l|l|} 
CH470101 & $>0$ \\
\end{tabular} & [if not missingAddSet] \\
\hline & & \begin{tabular}{|c|c|c|}
$\mathrm{CH} 470102$ & $<0$ \\
\end{tabular} & [if not missingAddset] \\
\hline & & $\mathrm{CH} 470103=0$ & [if not missingAddset] \\
\hline & & \begin{tabular}{l|l|l} 
CH470104 & combination \\
\end{tabular} & [if not missingAddSet] \\
\hline & & CH470105 Invalidvalue & [if not missingAddSet] \\
\hline & & \begin{tabular}{|c|c|c|} 
CH4 70106 & missingValue
\end{tabular} & \\
\hline \multirow[t]{2}{*}{ PA48 } & Grgeo & Additional Int Val4 & \\
\hline & CA4801 & content of the set & \\
\hline \multirow[t]{2}{*}{$B$} & & \begin{tabular}{|c|c|}
$\mathrm{CH} 480101$ & $>0$ \\
\end{tabular} & [if not missingAddSet] \\
\hline & & \begin{tabular}{l|l|}
$\mathrm{CH} 480102$ & $<0$ \\
\end{tabular} & [if not missingAddSet] \\
\hline
\end{tabular}




\begin{tabular}{|c|c|c|c|}
\hline & & \begin{tabular}{|l|l|l}
$\mathrm{CH} 480103$ & $=0$ \\
\end{tabular} & [if not missingAddset] \\
\hline & & \begin{tabular}{|l|l|}
$\mathrm{CH} 480104$ & combination \\
\end{tabular} & [if not missingAddset] \\
\hline & & \begin{tabular}{|l|l|} 
CH480105 & InvalidValue \\
\end{tabular} & [if not missingAddSet] \\
\hline & & \begin{tabular}{|l|l}
$\mathrm{CH} 480106$ & missingValue
\end{tabular} & \\
\hline PA49 & Grgeo & Additional Int val5 & \\
\hline & CA4901 & content of the set & \\
\hline$B$ & & \begin{tabular}{|l|l|}
$\mathrm{CH} 490101$ & $>0$ \\
\end{tabular} & [if not missingAddset] \\
\hline & & \begin{tabular}{|l|l|}
$\mathrm{CH} 490102$ & $<0$ \\
\end{tabular} & [if not missingAddSet] \\
\hline & & \begin{tabular}{|l|l}
$\mathrm{CH} 490103$ & $=0$ \\
\end{tabular} & [if not missingAddset] \\
\hline & & \begin{tabular}{|l|l|} 
CH490104 & combination \\
\end{tabular} & [if not missingAddSet] \\
\hline & & CH490105 InvalidValue & [if not missingAddSet] \\
\hline & & \begin{tabular}{|l|l} 
CH490106 & missingValue \\
\end{tabular} & \\
\hline PA50 & Grgeo & Additional Int Val6 & \\
\hline & CA5001 & content of the set & \\
\hline$B$ & & $|\mathrm{CH} 500101|>0$ & [if not missingAddset] \\
\hline & & CH500102 $<0$ & [if not missingAddset] \\
\hline & & CH500103 $=0$ & [if not missingAddSet] \\
\hline & & CH500104 combination & [if not missingAddSet] \\
\hline & & CH500105 InvalidValue & [if not missingAddSet] \\
\hline & & \begin{tabular}{|l|l|} 
CH500106 & missingValue \\
\end{tabular} & \\
\hline PA51 & Grgeo & Additional Real Vall & \\
\hline & CA5101 & content of the set & \\
\hline B & & \begin{tabular}{|l|l|} 
CH510101 & $>0$ \\
\end{tabular} & [if not missingAddSet] \\
\hline & & \begin{tabular}{|l|l|}
$\mathrm{CH} 510102$ & $<0$ \\
\end{tabular} & [if not missingAddSet] \\
\hline & & \begin{tabular}{|l|l|}
$\mathrm{CH} 510103$ & $=0$ \\
\end{tabular} & [if not missingAddSet] \\
\hline & & \begin{tabular}{|l|l} 
CH510104 & combination \\
\end{tabular} & [if not missingAddset] \\
\hline & & \begin{tabular}{|l|l|} 
CH510105 & InvalidValue \\
\end{tabular} & [if not missingAddSet] \\
\hline & & $\begin{array}{ll}\text { CH510106 missingValue } \\
\end{array}$ & \\
\hline PA52 & Grgeo & Additional Real Val2 & \\
\hline & CA5201 & content of the set & \\
\hline $\mathrm{B}$ & & \begin{tabular}{|l|l|} 
CH520101 & $>0$ \\
\end{tabular} & [if not missingAddSet] \\
\hline & & \begin{tabular}{|l|l|l|l|} 
CH520102 & $<0$ \\
\end{tabular} & [if not missingAddset] \\
\hline & & \begin{tabular}{|l|l}
$\mathrm{CH} 520103$ & $=0$ \\
\end{tabular} & [if not missingAddset] \\
\hline & & \begin{tabular}{|l|l|} 
CH520104 & combination \\
\end{tabular} & [if not missingAddSet] \\
\hline & & \begin{tabular}{|l|l|} 
CH520105 & InvalidValue \\
\end{tabular} & [if not missingAddSet] \\
\hline & & $\begin{array}{l}\text { CH520106 } \\
\text { missingValue }\end{array}$ & \\
\hline PA53 & Grgeo & Additional Real Val3 & \\
\hline & CA5301 & content of the set & \\
\hline $\mathrm{B}$ & & \begin{tabular}{|l|l|} 
CH530101 & $>0$ \\
\end{tabular} & [if not missingAddSet] \\
\hline & & \begin{tabular}{|c|c|}
$\mathrm{CH} 530102$ & $<0$ \\
\end{tabular} & [if not missingAddSet] \\
\hline & & CH530103 $=0$ & [if not missingAddset] \\
\hline & & CH530104 combination & [if not missingAddSet] \\
\hline & & CH530105 Invalidvalue & [if not missingAddset] \\
\hline & & \begin{tabular}{|l|l} 
CH530106 & missingValue \\
\end{tabular} & \\
\hline PA54 & Grgeo & Additional Unit & \\
\hline & CA5401 & content of the set & \\
\hline $\mathrm{B}$ & & CH540101 deg & [if not missingAddSet] \\
\hline & & \begin{tabular}{|l|l|} 
CH540102 & rad \\
\end{tabular} & [if not missingAddSet] \\
\hline & & \begin{tabular}{|l|l|} 
CH540103 & combination \\
\end{tabular} & [if not missingAddSet] \\
\hline & & \begin{tabular}{|l|l} 
CH540104 InvalidValue \\
\end{tabular} & [if not missingAddset] \\
\hline & & CH540105 missingValue & \\
\hline PA55 & Group & Grexc Def & \\
\hline & CA5501 & Definition in \{GROUP EXCIT & ATION \\
\hline & & CH550101 GROUP EXCITATION & [property G Excitation] \\
\hline
\end{tabular}




\begin{tabular}{|c|c|c|c|c|}
\hline & CA5502 & other val & Lue & \\
\hline \multirow[t]{2}{*}{$\mathrm{B}$} & & CH550201 & INVALID VALUE & [ERROR] \\
\hline & & CH550202 & errormissing & [ERROR] \\
\hline \multirow{2}{*}{ PA56 } & Grexc & Gramp Exed & & \\
\hline & CA5601 & Definitiol & on in $\{$ AMPLITUDE & \\
\hline \multirow[t]{3}{*}{$\mathrm{B}$} & & CH560101 & AMPLITUDE & [property G Amplitude] \\
\hline & CA5602 & other val & Lue & \\
\hline & & CH560201 & INVALID VALUE & [ERROR] \\
\hline $\bar{B}$ & & CH560202 & errorMissing & [ERROR] \\
\hline \multirow[t]{2}{*}{ PA57 } & Grexc & Gramp Exec & c Def & \\
\hline & CA5701 & Definitio & on in $\{$ UNIFORM, $S E$ & OND \\
\hline \multirow[t]{4}{*}{ B } & & CH570101 & UNIFORM & $\begin{array}{l}\text { [if G_Amplitude, property } \\
\text { gramp uniform] }\end{array}$ \\
\hline & & CH570102 & SECOND & $\begin{array}{l}\text { [if G_Amplitude, property } \\
\text { gramp-second] }\end{array}$ \\
\hline & CA5702 & $\begin{array}{l}\text { other } \\
\text { value }\end{array}$ & & \\
\hline & & CH570201 & INVALID VALUE & [ERROR] [if G Amplitude] \\
\hline $\mathrm{B}$ & & CH570202 & errorMissing & [ERROR] [if G Amplitude] \\
\hline \multirow[t]{3}{*}{ PA58 } & Grexc & Gramp Exec & C Vall & \\
\hline & CA5801 & real numb & & \\
\hline & & CH580101 & $=0$ & [if gramp uniform or gramp second] \\
\hline \multirow[t]{5}{*}{$B$} & & $\mathrm{CH} 580102$ & $>0$ & [if gramp uniform or gramp second] \\
\hline & & CH580103 & $<0$ & [if gramp uniform or gramp second] \\
\hline & CA5802 & Other val & lue & \\
\hline & & CH580201 & non-number & $\begin{array}{l}\text { [ERROR] [if gramp_uniform or } \\
\text { gramp second] }\end{array}$ \\
\hline & & CH580202 & errorMissing & [ERROR] [if gramp second] \\
\hline B & & CH580203 & missingValue & [if gramp uniform] \\
\hline \multirow[t]{2}{*}{ PA59 } & Grexc & Gramp Exec & C Def2 & \\
\hline & CA5901 & Definitio & on in $\{\mathrm{P} 1 \mathrm{VAL}\}$ & \\
\hline \multirow[t]{3}{*}{$B$} & & CH590101 & P1 VAL & [if gramp second] \\
\hline & CA5902 & Other val & lue & \\
\hline & & CH590201 & INVALID VALUE & [ERROR] [if gramp second] \\
\hline \multirow[t]{2}{*}{$\mathrm{B}$} & & CH590202 & errorMissing & [ERROR] [if gramp second] \\
\hline & & CH590203 & missingValue & \\
\hline \multirow[t]{3}{*}{ PA60 } & Grexc & Gramp Exed & C Val2 & \\
\hline & CA6001 & real numb & & \\
\hline & & CH600101 & $=0$ & [if gramp second] \\
\hline \multirow[t]{4}{*}{$\mathrm{B}$} & & $\mathrm{CH} 600102$ & $>0$ & [if gramp second] \\
\hline & & $\mathrm{CH} 600103$ & $<0$ & [if gramp second] \\
\hline & CA6002 & Other val & lue & \\
\hline & & CH600201 & non-number & [ERROR] [if gramp second] \\
\hline \multirow[t]{2}{*}{$\mathrm{B}$} & & $\mathrm{CH} 600202$ & errorMissing & [ERROR] [if gramp second] \\
\hline & & $\mathrm{CH} 600203$ & missingValue & \\
\hline \multirow[t]{2}{*}{ PA61 } & Grexc & Gramp_Exed & C_Def3 & \\
\hline & CA6101 & Definitio & on in $\{$ P2 2 VAL $\}$ & \\
\hline \multirow[t]{3}{*}{$\mathrm{B}$} & & CH610101 & P2 VAL & [if gramp second] \\
\hline & CA6102 & Other val & lue & \\
\hline & & CH610201 & INVALID VALUE & [ERROR] [if gramp second] \\
\hline \multirow[t]{2}{*}{$\mathrm{B}$} & & $\mathrm{CH} 610202$ & errorMissing & [ERROR] [if gramp_second] \\
\hline & & CH610203 & missingValue & \\
\hline A62 & & Gramp Exed & C Val3 & \\
\hline
\end{tabular}




\begin{tabular}{|c|c|c|c|}
\hline & & \begin{tabular}{|l|l|}
$\mathrm{CH} 620101$ & $=0$ \\
\end{tabular} & [if gramp second] \\
\hline \multirow[t]{5}{*}{ B } & & \begin{tabular}{|l|l|}
$\mathrm{CH} 620102$ & $>0$ \\
\end{tabular} & [if gramp second] \\
\hline & & \begin{tabular}{|l|l|}
$\mathrm{CH} 620103$ & $<0$ \\
\end{tabular} & [if gramp second] \\
\hline & CA6202 & Other value & \\
\hline & & \begin{tabular}{|l|l|} 
CH620201 & non-number \\
\end{tabular} & [ERROR] [if gramp second] \\
\hline & & CH620202 lerrorMissing & [ERROR] [if gramp second] \\
\hline $\mathrm{B}$ & & CH620203 missingValue & \\
\hline \multirow[t]{2}{*}{ PA63 } & Grexc & Gramp Exec Def4 & \\
\hline & CA6301 & Definition in \{ 21 VAL \} & \\
\hline \multirow[t]{4}{*}{$\mathrm{B}$} & & \begin{tabular}{|l|ll} 
CH630101 & VAL \\
\end{tabular} & [if gramp second] \\
\hline & CA 6302 & Other value & \\
\hline & & \begin{tabular}{|l|l|l}
$\mathrm{CH} 630201$ & INVALID VALUE \\
\end{tabular} & [ERROR] [if gramp second] \\
\hline & & CH630202 errorMissing & [ERROR] [if gramp second] \\
\hline $\mathrm{B}$ & & \begin{tabular}{|l|l} 
CH630203 & missingValue \\
\end{tabular} & \\
\hline \multirow[t]{3}{*}{$\mathrm{PA} 64$} & Grexc & Gramp_Exec_Val4 & \\
\hline & CA6401 & real number & \\
\hline & & $\mathrm{CH} 640101=0$ & [if gramp second] \\
\hline \multirow[t]{5}{*}{$\mathrm{B}$} & & $\mathrm{CH} 640102>0$ & [if gramp second] \\
\hline & & CH640103 $<0$ & [if gramp second] \\
\hline & CA6402 & Other value & \\
\hline & & \begin{tabular}{|l|l|} 
CH640201 & non-number \\
\end{tabular} & [ERROR] [if gramp second] \\
\hline & & \begin{tabular}{|l|l|} 
CH640202 & errorMissing \\
\end{tabular} & [ERROR] [if gramp second] \\
\hline $\mathrm{B}$ & & $\begin{array}{l}\text { CH640203 } \\
\text { missingValue }\end{array}$ & \\
\hline \multirow[t]{5}{*}{ PA65 } & Grexc & Gramp Exec Def5 & \\
\hline & CA6501 & Definition in $\{$ Q2 VAL $\}$ & \\
\hline & & \begin{tabular}{|l|l|l|} 
CH650101 & Q2 VAI \\
\end{tabular} & [if gramp second] \\
\hline & CA6502 & Other value & \\
\hline & & \begin{tabular}{|l|l|} 
CH650201 & INVALID VALUE \\
\end{tabular} & [if gramp second] \\
\hline $\mathrm{B}$ & & \begin{tabular}{|l|l|} 
CH650202 & missingValue \\
\end{tabular} & \\
\hline \multirow{3}{*}{ PA66 } & Grexc & Gramp Exec Val5 & \\
\hline & CA6601 & real number & \\
\hline & & \begin{tabular}{|l|l|} 
CH660101 & $=0$ \\
\end{tabular} & [if gramp second] \\
\hline \multirow[t]{5}{*}{$\mathrm{B}$} & & \begin{tabular}{|l|l|}
$\mathrm{CH} 660102$ & $>0$ \\
\end{tabular} & [if gramp second] \\
\hline & & \begin{tabular}{|l|l|}
$\mathrm{CH} 660103$ & $<0$ \\
\end{tabular} & [if gramp second] \\
\hline & CA6602 & Other value & \\
\hline & & \begin{tabular}{|l|l|} 
CH660201 & non-number \\
\end{tabular} & [ERROR] [if gramp second] \\
\hline & & \begin{tabular}{|l|l|} 
CH660202 & errorMissing \\
\end{tabular} & [ERROR] [if gramp second] \\
\hline B & & \begin{tabular}{|l|l} 
CH660203 & missingValue \\
\end{tabular} & \\
\hline \multirow[t]{2}{*}{ PA67 } & Grexc & Gramp Exec Unit & \\
\hline & CA6701 & Definition in $\{\mathrm{m}, \mathrm{mm}, \mathrm{dm}, \mathrm{cm}$ & LINEAR, POWER, dB \} \\
\hline \multirow[t]{9}{*}{$\mathrm{B}$} & & \begin{tabular}{|l|l|} 
CH670101 & LINEAR \\
\end{tabular} & [if gramp uniform] \\
\hline & & \begin{tabular}{|l|l|} 
CH670102 & POWER \\
\end{tabular} & [if gramp uniform] \\
\hline & & \begin{tabular}{|l|l|}
$\mathrm{CH} 670103$ & $\mathrm{~dB}$ \\
\end{tabular} & [if gramp uniform] \\
\hline & & \begin{tabular}{|l|l|l|l}
$\mathrm{CH} 670104$ & $\mathrm{~mm}$ \\
\end{tabular} & [if gramp second] \\
\hline & & \begin{tabular}{|l|l|l}
$\mathrm{CH} 670105$ & $\mathrm{dm}$ \\
\end{tabular} & [if gramp second] \\
\hline & & \begin{tabular}{|l|l|}
$\mathrm{CH} 670106$ & $\mathrm{~cm}$ \\
\end{tabular} & [if gramp second] \\
\hline & & \begin{tabular}{|l|l|}
$\mathrm{CH} 670107$ & $\mathrm{~m}$ \\
\end{tabular} & [if gramp second] \\
\hline & $\overline{\mathrm{CA}} 6702$ & \begin{tabular}{|l|l|} 
Other & \\
value
\end{tabular} & \\
\hline & & \begin{tabular}{|l|l|} 
CH670201 & INVALID_VALUE \\
\end{tabular} & $\begin{array}{l}\text { [ERROR] [if gramp_uniform or } \\
\text { gramp second] }\end{array}$ \\
\hline B & & \begin{tabular}{|l|l} 
CH670202 & missingValue \\
\end{tabular} & \\
\hline \multirow[t]{2}{*}{ PA68 } & Grexc & Grpha Exec & \\
\hline & & Definition in $\{$ PHASE $\}$ & \\
\hline
\end{tabular}




\begin{tabular}{|c|c|c|c|c|}
\hline B & & CH680101 & PHASE & [property phase] \\
\hline & CA6802 & other val & & \\
\hline B & & $\mathrm{CH} 680201$ & INVALID VALUE & [ERROR] \\
\hline PA69 & Grexc & Grpha Def & & \\
\hline & CA6901 & Definitio & on in i UNIFORM, & SECOND， ROTATION， POINT ING $\}$ \\
\hline $\mathrm{B}$ & & $\mathrm{CH} 690101$ & UNIFORM & [[property grpha uniform] \\
\hline & & CH690102 & SECOND & [property grpha second] \\
\hline & & $\mathrm{CH} 690103$ & ROTATION & [property grpha rotation] \\
\hline & & CH690104 & POINTING & [property grpha pointing] \\
\hline & CA6902 & other val & & \\
\hline & & CH690201 & INVALID VALUE & \\
\hline B & & CH 690202 & lerrorMissing & \\
\hline PA70 & Grexc & Grpha_Exe & C Def & \\
\hline & CA7001 & Definitic & on in $\{\mathrm{CW}, \mathrm{CCW}$, & U, THETA \} \\
\hline B & & $\mathrm{CH} 700101$ & $\mathrm{CW}$ & [if grpha rotation] \\
\hline & & $\mathrm{CH} 700102$ & $\mathrm{CCW}$ & [if grpha rotation] \\
\hline & & $\mathrm{CH} 700103$ & $\mathrm{U}$ & $\begin{array}{l}\text { [if grpha_pointing, property } \\
\text { pointing_axis] }\end{array}$ \\
\hline & & $\mathrm{CH} 700104$ & THETA & $\begin{array}{l}\text { [if grpha_pointing, property } \\
\text { pointing angle] }\end{array}$ \\
\hline & CA7002 & other val & & \\
\hline & & $\mathrm{CH} 700201$ & INVALID VALUE & [ERROR] \\
\hline $\mathrm{B}$ & & $\mathrm{CH} 700202$ & missingValue & \\
\hline PA71 & Grexc & Grpha Exe & C Vall & \\
\hline & CA7101 & values in & {$[-1.1]$} & \\
\hline & & $\mathrm{CH} 710101$ & $=0$ & [if not grpha uniform] \\
\hline & & $\mathrm{CH} 710102$ & $=-1$ & [if pointing axis] \\
\hline & & $\mathrm{CH} 710103$ & $=1$ & [if pointing axis] \\
\hline & & $\mathrm{CH} 710104$ & $(-1,0)$ & [if pointing axis] \\
\hline $\mathrm{B}$ & & $\mathrm{CH} 710105$ & $(0,1)$ & [if pointing axis] \\
\hline & CA7102 & real numb & & \\
\hline B & & CH710201 & $>0$ & $\begin{array}{l}\text { [if not (grpha uniform or } \\
\text { pointing axis)] }\end{array}$ \\
\hline & & $\mathrm{CH} 710202$ & $<0$ & $\begin{array}{l}\text { [if not (grpha uniform or } \\
\text { pointing axis)] }\end{array}$ \\
\hline & CA7103 & other val & & \\
\hline & & CH710301 & $<-1$ & [ERROR] [if pointing axis] \\
\hline $\mathrm{B}$ & & $\mathrm{CH} 710302$ & $\geq 1$ & [ERROR] [if pointing axis] \\
\hline & & $\mathrm{CH} 710303$ & non-number & [ERROR] [if not grpha uniform] \\
\hline & & $\mathrm{CH} 710304$ & missingvalue & \\
\hline PA72 & Grexc_ & Grpha Exe & C_Def1 & \\
\hline & CA7201 & Definitio & on in $\{\mathrm{P} 1$ VAL, ST & $\mathrm{EP}, \mathrm{V}, \mathrm{PHI}\}$ \\
\hline B & & CH720101 & P1 VAL & [if grpha_second] \\
\hline & & CH720102 & STEP & [if grpha rotation] \\
\hline & & CH720103 & $\mathrm{V}$ & [if pointing axis] \\
\hline & & CH720104 & PHI & [if pointing angle] \\
\hline & CA7202 & other val & Lue & \\
\hline & & $\mathrm{CH} 720201$ & INVALID VALUE & [ERROR] \\
\hline $\mathrm{B}$ & & $\mathrm{CH} 720202$ & missingValue & \\
\hline PA73 & Grexc & Grpha Exe & C Val2 & \\
\hline & CA7301 & values in & {$[-1,1]$} & \\
\hline & & $\mathrm{CH} 730101$ & $=0$ & $\begin{array}{l}\text { [if not (grpha_uniform or } \\
\text { pointing angle] }\end{array}$ \\
\hline & & $\mathrm{CH} 730102$ & $=-1$ & [if pointing axis] \\
\hline
\end{tabular}




\begin{tabular}{|c|c|c|c|c|}
\hline & & CH730103 & $=1$ & [if pointing axis] \\
\hline \multirow[t]{3}{*}{$B$} & & CH730104 & $(-1,0)$ & [if pointing axis] \\
\hline & & CH730105 & $(0,1)$ & [if pointing axis] \\
\hline & CA7302 & real numb & ser & \\
\hline \multirow[t]{4}{*}{ B } & & CH730201 & $>0$ & $\begin{array}{l}\text { [if not (grpha uniform or } \\
\text { grpha pointing)] }\end{array}$ \\
\hline & & $\mathrm{CH} 730202$ & $<0$ & $\begin{array}{l}{[\text { if not (grpha uniform or }} \\
\text { grpha pointing)] }\end{array}$ \\
\hline & CA7303 & other val & Lue & \\
\hline & & CH730301 & $<-1$ & [ERROR] [if pointing axis] \\
\hline \multirow[t]{3}{*}{$\mathrm{B}$} & & $\mathrm{CH} 730302$ & $>1$ & [ERROR] [if pointing axis] \\
\hline & & CH730303 & non-number & $\begin{array}{l}\text { [ERROR] [if not (grpha_uniform or } \\
\text { pointing angle] }\end{array}$ \\
\hline & & $\mathrm{CH730304}$ & missingValue & \\
\hline \multirow[t]{2}{*}{ PA74 } & Grexc & Grpha Exec & c Unit1 & \\
\hline & CA7401 & Definitio & on in $\{$ deg, rad $\}$ & \\
\hline B & & CH740101 & deg & [if grpha rotation] \\
\hline & & $\mathrm{CH} 740102$ & rad & [if grpha rotation] \\
\hline & CA7402 & other val & lue & \\
\hline & & CH740201 & INVALID VALUE & [ERROR] \\
\hline B & & CH740202 & missingValue & \\
\hline \multirow[t]{3}{*}{ PA75 } & Grexc & Grpha Exer & C Def2 & \\
\hline & CA7501 & Definitio & $\mathrm{Sn}$ in \{P2 VAI, STI & \\
\hline & & CH750101 & P2 VAI & [if grpha second] \\
\hline \multirow[t]{3}{*}{$\mathrm{B}$} & & CH750102 & START & [if grpha rotation] \\
\hline & CA7502 & other val & Lue & \\
\hline & & CH750201 & INVALID VALUE & [ERROR] \\
\hline B & & $\mathrm{CH} 750202$ & missingValue & \\
\hline \multirow[t]{3}{*}{ PA76 } & Grexc & Grpha Exer & C Val3 & \\
\hline & CA7601 & real numb & per & \\
\hline & & CH760101 & $=0$ & $\begin{array}{l}\text { [if not (grpha uniform or } \\
\text { grpha pointing)] }\end{array}$ \\
\hline \multirow[t]{4}{*}{ B } & & $\mathrm{CH} 760102$ & $>0$ & $\begin{array}{l}\text { [if not (grpha_uniform or } \\
\text { grpha pointing)] }\end{array}$ \\
\hline & & CH760103 & $<0$ & $\begin{array}{l}\text { [if not (grpha_uniform or } \\
\text { grpha pointing)] }\end{array}$ \\
\hline & CA7602 & Jother val & Lue & \\
\hline & & $\mathrm{CH760201}$ & non-number & $\begin{array}{l}\text { [ERROR] [if not (grpha_uniform or } \\
\text { grpha_pointing)] }\end{array}$ \\
\hline B & & $\mathrm{CH} 760202$ & missingValue & \\
\hline \multirow[t]{3}{*}{ PA77 } & Grexc & Grpha Exe & c Def3 & \\
\hline & CA7701 & Definitio & $\mathrm{on}$ in $\{Q 1$ VAL, STE & \\
\hline & & CH770101 & Q1 VAL & [if grpha second] \\
\hline \multirow[t]{3}{*}{$B$} & & $\mathrm{CH} 770102$ & STEP & [if grpha rotation] \\
\hline & CA7702 & other nom & ninal value & \\
\hline & & $\mathrm{CH} 770201$ & INVAL ID VALUE & [ERROR] \\
\hline B & & $\mathrm{CH} 770202$ & missingvalue & \\
\hline \multirow[t]{3}{*}{ PA78 } & Grexc & Grpha Exe & C Val4 & \\
\hline & CA7801 & real numb & & \\
\hline & & $\mathrm{CH} 780101$ & $=0$ & $\begin{array}{l}\text { [if not (grpha_uniform or } \\
\text { grpha rotation or grpha pointing)] }\end{array}$ \\
\hline \multirow[t]{2}{*}{ B } & & $\mathrm{CH} 780102$ & $>0$ & $\begin{array}{l}\text { [if not (grpha_uniform or } \\
\text { grpha rotation or grpha pointing)] }\end{array}$ \\
\hline & & $\mathrm{CH} 780103$ & $<0$ & $\begin{array}{l}\text { [if not (grpha_uniform or } \\
\text { grpha rotation or grpha pointing)] }\end{array}$ \\
\hline
\end{tabular}




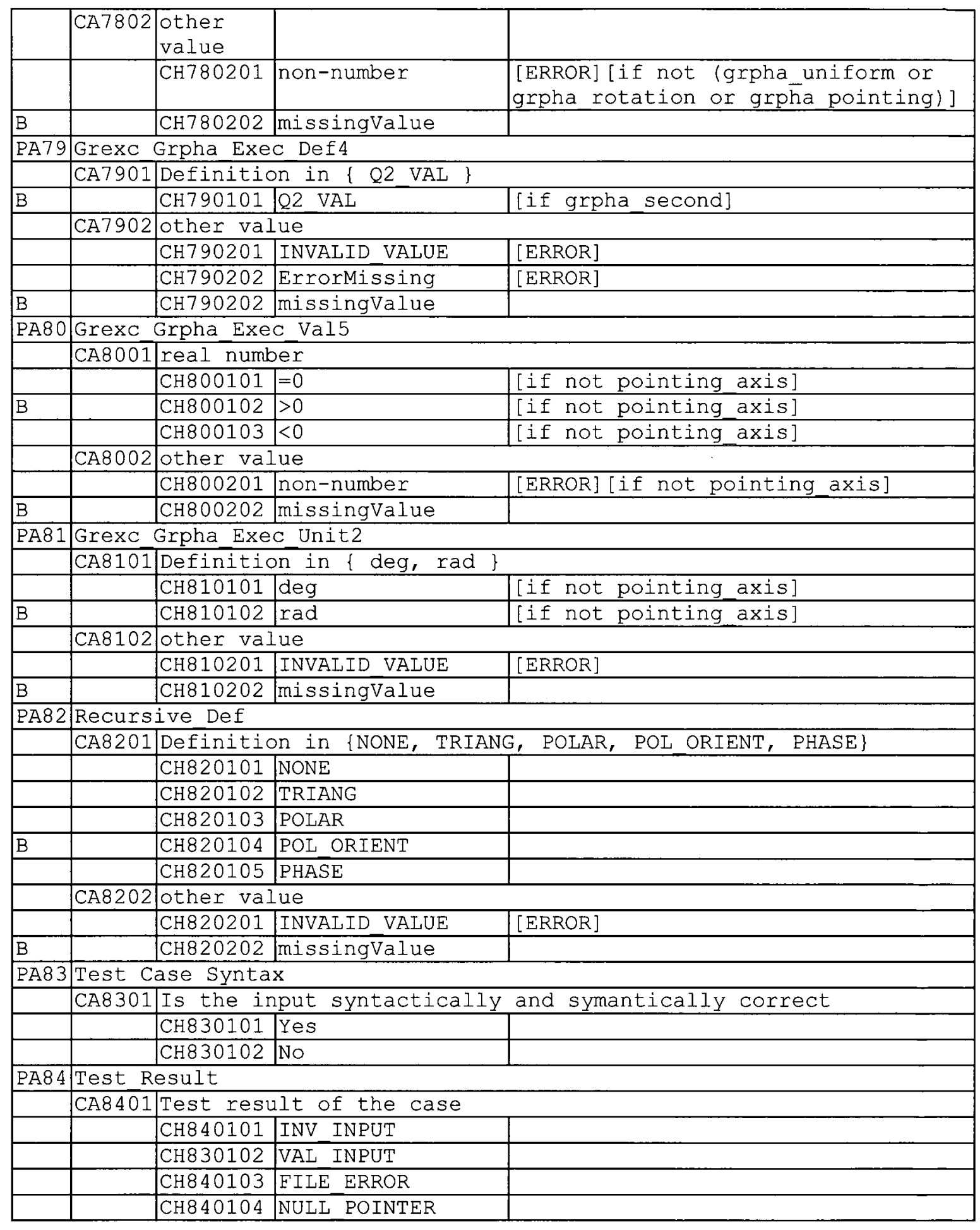

